\title{
42nd ESCP symposium on clinical pharmacy: implementation of pharmacy practice; Prague, Czech Republic, 16-18 October 2013
}

CC Koninklijke Nederlandse Maatschappij ter bevordering der Pharmacie 2013

\section{OCI Pharmacotherapy}

\section{PT01}

Low dose of rituximab: a novel therapy option for the treatment of Graves' orbitopathy

G. Ulmann ${ }^{1}$, A. Cransac1, M.-T. Baylatry ${ }^{1, *}$, S. Rivière ${ }^{2}$, M. Berche ${ }^{3}$, L. Du Pasquier ${ }^{4}$, A.-C. Joly ${ }^{1}$, E. Héron ${ }^{4}$

${ }^{1}$ Pharmacy, ${ }^{2}$ Internal Medicine, Saint-Antoine Hospital (East Parisian University Hospitals, AP-HP), ${ }^{3}$ Ophthalomology, ${ }^{4}$ Internal Medicine, Quinze-Vingts National Ophthalmology Hospital, Paris, France

Background and Objective: Graves' orbitopathy (GO) is an autoimmune inflammatory disease related to a thyroidal disorder. Symptoms include exophthalmia, impaired vision, diplopia and even blindness due to the compression of the optic nerve. Usual treatments of GO: immunosuppressive therapies (steroids, cyclosporin), orbital irradiation and surgical decompression are limited due to recurrences. Some studies [1-3] have suggested that rituximab (RTX), an antiCD20 monoclonal antibody, at a dose of $1,000 \mathrm{mg}$, might be effective in active GO by a B lymphocyte depletion. Preliminary data [4] suggests that $100 \mathrm{mg}$ of RTX may induce effective peripheral B lymphocyte depletion and long term remission of GO. We report 8 cases of active GO treated with $100 \mathrm{mg}$ of RTX.

Setting and Method: Case report from Internal Medicine and Pharmacy departments. Safety and efficacy results of $100 \mathrm{mg}$ RTX in active GO treatment are presented.

Main outcome measures: Efficacy evaluation: clinical activity score (CAS). CAS is a specific score for GO that consists in 10 items related to the 4 clinical signs of inflammation (pain, redness, swelling and impaired function). For each item present, 1 point is given and the addition of these points leads to CAS (range 0-10) [5]. Safety evaluation: occurrence of adverse events (infection, hematologic and cardio-vascular events).

Results: 8 patients ( 2 men, 6 women, mean age 45 years) with active GO steroid-resistant or intolerant were treated between April 2012 and July 2013 with $100 \mathrm{mg}$ of RTX. 6 patients received 2 infusions at days 1 and 15, 1 patient received 1 infusion and 1 patient is presently waiting for the second infusion. Mean CAS for the 7 fully evaluated patients was $3.8 / 10$ before infusion and dropped to $0.8 / 10$ after. No adverse event was observed during and after RTX infusion except in one patient: an episode of palpitations was described the following day. This patient did not receive the second infusion. After a median follow-up of 2.3 months (after the first infusion), no over adverse events were declared.

Conclusions: Low dose of RTX seems to be efficient and well tolerated for treating GO thus confirming preliminary data. If these findings are confirmed in a larger cohort, the RTX dose of $1,000 \mathrm{mg}$ currently used to treat patients could be reduced to $100 \mathrm{mg}$.

[1] Salvi M., Rituximab for the treatment of Graves' Orbitopathy, 2011

[2] Laszlo H., Targeted biological therapies for Graves' diseases and thyroid associated ophtalmopathy. Focus on B cell depletion with Rituximab, 2011

[3] Pandit A., Rituximab therapy in steroid-resistant severe hypothyroid Grave's ophtalmopathy, 2013

[4] Salvi M., Small dose of rituximab for Graves Orbitopathy: new insights into the mechanism of action, 2012

[5] Mourits M., Clinical activity score as a guide in the management of patients with Graves' ophtalmopathy, 1997

Disclosure of Interest: None Declared.

\section{PT02}

Tolvaptan versus urea in inappropriate secretion syndrome of hormone antidiuretic: a case report

I. Gómez Valbuena ${ }^{1, *}$, D. Alioto ${ }^{1}$, L. Alonso Pérez ${ }^{1}$, I. Cañamares Orbis ${ }^{1}$, J. M. Caro Teller ${ }^{1}$, I. Escribano Valenciano' ${ }^{1}$, J. M. Ferrari Piquero ${ }^{1}$

${ }^{1}$ Hospital Universitario 12 de Octubre, Madrid, Spain

Background and Objective: Syndrome of Inappropriate Antidiuretic Hormone Secretion (SIADH) is a disease caused by excessive release of vasopressin. Currently are being debated therapeutic options to treat hyponatremia of SIADH, evidence on the cost-effectiveness and safety of receptor antagonists of vasopressin, like tolvaptan.

The goal of this study is describe a case in which the change from tolvaptan treatment with urea in the SIADH allowed to keep the answer to a much lower cost.

Results: A 83-year woman patient was admitted to hospital due to fall at her domicile. During the exploration, a severe hyponatremia was diagnosed $(108 \mathrm{mEq} / \mathrm{L})$. 
Before SIADH's suspicion three different treatments were prescribed during the admission:

1. Water restriction and saline 7 days.

2. Tolvaptan at a dose of $15 \mathrm{mg}$ every $24 \mathrm{~h}$ for two days followed by $15 \mathrm{mg}$ every $48 \mathrm{~h}$ until day 16 of treatment. At that moment the pattern of treatment was changed to $15 \mathrm{mg}$ daily. The patient received a total of 14 doses.

3. 15 grams of Urea envelopes every $12 \mathrm{~h}$ prepared by Pharmacotechnology area of hospital pharmacy department.

The effect of the different treatments was compared, as for the normalization of the blood levels of sodium during hospital admission, as well as the cost.

Serum sodium levels of the patient during saline water restriction remain around $120 \mathrm{mEq} / \mathrm{L}$. During treatment with tolvaptan the average level of the patient's serum sodium was $129.67 \mathrm{mEq} / \mathrm{L}$. Throughout urea treatment, the patient remained stable and even had a slight increase in the average level of serum sodium $(134,25 \mathrm{mEq} / \mathrm{L})$. The patient was discharged after 48 days administration urea long-term therapy.

The daily cost of tolvaptan doses, used in the patient, is between 33.5 and 69 euros each day, the urea treatment cost would be around 0.30 euros per day.

Conclusions: In cases such as these, the use of urea prepared as master formula for the area of the Pharmacy Service as alternative treatment therapy tolvaptan in SIADH can get the same result that tolvaptan in normalization of serum sodium, a markedly lower cost. Disclosure of Interest: None Declared.

\section{PT03}

A 12-month retrospective study of clinical and therapeutic management of Clostridium difficile infection

S. Santana Martínez ${ }^{1, *}$, M. Beltrán García ${ }^{1}$, E. Romero Carreño ${ }^{1}$, M. Moya Martín ${ }^{1}$, M. Nuñez Nuñez ${ }^{1}$, F. Caracuel de Castro ${ }^{1}$

${ }^{1}$ Hospital Pharmacy, Hospital Universitario Virgen Macarena, Sevilla, Spain

Background and Objective: Clostridium difficile remains the leading cause of diarrhea associated with medical treatment and is becoming increasingly important as a pathogen in the community. Data has been published which reports on the decreased effectiveness of metronidazole in the treatment of $C$. difficile infection (CDI). This review aims to determine the current status of CDI in our hospital and the adequacy of the existing treatment protocol.

Setting and Method: Literature review. We selected all patients diagnosed with CDI during 2012 and consulted their medical histories.

Results: 17 patients ( 9 women, $58.7 \pm 26$ years). In $65 \%$ of cases, the antibiotic therapy considered the cause of the CDI was discontinued and the treatment was performed on $82 \%$ the day when the suspicion of CDI was confirmed. Regarding risk factors, in addition to age ( $65 \%>65$ years), $76 \%$ of patients were being treated concurrently with proton pump inhibitors, $12 \%$ had undergone some type of surgery and another $59 \%$ had concurrent antibiotic therapy. 6 were hospitalized in the previous 30 days and 11 were treated with antibiotics or chemoterapy within 8 weeks prior to the episode of CDI. 14 were diagnosed using CDI toxin detection in feces and 3 by colonoscopy. Considering the classification criteria, 14 were classified as mild-moderate and 3 as severe. Meanwhile, 15 patients received the treatment corresponding to a mild-moderate CDI consisting in $500 \mathrm{mg} / 8 \mathrm{~h}$ metronidazole for 10 days, while only 12 patients completed treatment. 2 patients were treated simultaneously with intravenous metronidazole and oral vancomycin, according to the criteria of severe CDI. Thus, $35 \%$ did not adapt to the treatment protocol: in 3 cases the treatment was chosen inappropriately and in another 3 the duration of treatment was shorter than recommended. In all cases the chosen treatment was effective. Relapses were not observed during the month following completement of treatment.

Conclusions: The effectiveness of treatment of CDI is appropriate in the studied period, although only $65 \%$ adhered to the protocol of our hospital. In view of the results and the number of recurrences, a longer study period would be appropriate to make conclusions regarding treatment effectiveness.

Disclosure of Interest: None Declared.

\section{PT04}

Efficacy of treatment with inhaled colistin in patients with bronchiectasis colonized by Pseudomonas aeruginosa non cystic fibrosis.

A. Trujillano Ruiz ${ }^{1, *}$, M. J. Avilés Inglés ${ }^{2}$, T. Antequera Lardón', L. Rentero Redondo ${ }^{1}$, P. Méndez Martínez ${ }^{2}$, J. M. Alonso Herreros ${ }^{1}$

${ }^{1}$ Servicio de Farmacia, ${ }^{2}$ Servicio de Neumología, Hospital General Universitario Reina Sofía, Murcia, Spain

Background and Objective: Taking into account that treatment with inhaled colistin (IC) requires learning and constancy by the patient and that it is also long and expensive; our aim has been to evaluate whether the treatment is effective in the eradication of Pseudomonas aeruginosa in bronchiectasis non cystic fibrosis (CF), as well as the improvement in their quality of life.

Setting and Method: Retrospective observational study conducted from January 2010 until May 2013. We collected demographic, clinical and microbiological diagnosis and indication data, considering in each case whether it was initial colonization (first positive crops of the same bacteria not previously isolated), intermittent (positive and negative crops for the same bacteria) or chronic (3 or more positive crops for the same bacteria with at least 1 month among them and in a period of 6 months).

At the end of treatment, effectiveness was verified by evaluating the negative crops and adverse effects; and quality of life by measuring the use of antibiotics and hospitalizations pretreatment and posttreatment with IC.

The data were obtained from the hospital computer applications ServoLab ${ }^{\circledR}$, Savac $^{\circledR}$ and EDC02 ${ }^{\circledR}$.

Results: We included 44 patients who started treatment with IC with an average age of $67.8 \pm 16.5$ years, $52.3 \%$ were women. The total patients were diagnosed with non-CF bronchiectasis. Of these, $29.5 \%$ also had COPD, 18.2 and $20.5 \%$ asthma postuberculosis injuries. As for the type of colonization, in $12(27.3 \%)$ was initial in $5(11.4 \%)$ intermittent and in $18(41 \%)$ chronic. The rest $(20.5 \%)$ had no crops and treatment was started empirically. All crops were sensitive to colistin except in two cases that had not been tested. At the time of data collection 26 patients completed the treatment (59.1\%) of which 7 were for abandonment ( 6 for adverse reactions and one for difficulty in handling the device). Regarding adverse reactions, were highlighted in 4 of the patients with respiratory, thoracic and mediastinal disorders (the most frequents according to the technical data sheet).

The average duration of treatment was $7.5 \pm 6.3$ months and 10 negativized the crops ( 4 with initial, 1 with intermittent and 5 with chronic colonization) with an average of $4.7 \pm 2.8$ crops posttreatment.

The average of pretreatment income was $2.5 \pm 2.8$ compared with $0.9 \pm 1.2$ after treatment, decreasing the number of admissions in 16 patients $(61.5 \%)$. The average of drugs that were previously taken 
prior to treatment with IC was $5.0 \pm 4.0$ while after treatment was $2.2 \pm 1.9$.

Conclusions: The proportion of patients who negativized crops with initial colonization is relevant $(33.3 \%)$, thus conclude that early beginning improves efficiency.

The decreased use of antibiotics and hospital admission along with few adverse reactions, demonstrate the improvement in the quality of life of patients.

Disclosure of Interest: None Declared.

\section{PT05}

NSAID-antihypertensive drug interactions: which outpatients are at risk for a rise in systolic blood pressure

A. Floor-Schreudering 1,2,*, P. De Smet ${ }^{2}$, H. Buurma ${ }^{1}$, C. Kramers ${ }^{3,4}$, C. Tromp ${ }^{5}$, S. V. Belitser ${ }^{6}$, M. Bouvy ${ }^{1,6}$

${ }^{1}$ SIR Institute for Pharmacy Practice and Policy, Leiden, ${ }^{2}$ Departments of Clinical Pharmacy and IQ Healthcare, ${ }^{3}$ Departments of General Internal Medicine and Pharmacology and Toxicology, Radboud University Nijmegen Medical Centre, ${ }^{4}$ Department of Clinical Pharmacy, Canisius Wilhelmina Ziekenhuis, Nijmegen, ${ }^{5}$ Health Base Foundation, Houten, ${ }^{6}$ Division Pharmacoepidemiology \& Clinical Pharmacology, Utrecht Institute for Pharmaceutical Sciences (UIPS), Utrecht University, Utrecht, Netherlands

Background and Objective: Management guidelines for drug-drug interactions (DDIs) between NSAIDs and antihypertensives recommend blood pressure monitoring in hypertensive patients. We measured the short-term effect of initiating NSAIDs on the systolic blood pressure (SBP) in users of antihypertensives aiming to investigate which outpatients are at risk for an increase in SBP in daily clinical practice.

Setting and Method: A cohort study with a nested case-control design in Dutch community pharmacies. Patients with a DDI alert for a newly initiated NSAID and antihypertensive were interviewed and their SBP was measured at $\mathrm{T}=0, \mathrm{~T}=1$ (after 1 week), $\mathrm{T}=2$ (after 2 weeks). Main outcome measures: We evaluated risk factors for exceeding a predefined limit of change (PLoC) in SBP $(\geq 10 \mathrm{mmHg}$ to $\geq 140 \mathrm{mmHg}$ ) at $\mathrm{T} 1$ and $\mathrm{T} 2$ versus $\mathrm{T} 0$.

Results: For 112 patients the SBP at T0 was measured. Two patients were excluded (T0 SBP $\geq 180 \mathrm{mmHg}$ ). PLoC was exceeded in 10 patients $(10.4 \%)$ at $\mathrm{T} 1$ and in 7 patients $(8.0 \%)$ at T2. Patients using etoricoxib (OR 21.0; $95 \%$ CI 3.7-120.6) and patients using $>1$ defined daily dose of an NSAID (OR 3.3; $95 \%$ CI 1.1-10.0) were at increased risk of a rise in SBP.

Conclusions: A newly initiated NSAID has an immediate clinically relevant effect on SBP in some users of antihypertensives. Management guidelines for NSAID-antihypertensive DDIs should advice SBP monitoring before and after initiation of an NSAID or intensification of NSAID therapy. Monitoring is especially relevant in patients prescribed high dosages of NSAIDS. Etoricoxib should not be used in hypertensive patients.

Disclosure of Interest: None Declared.

\section{PT06}

Feedback of the experience of the use of argatroban

L. Lehmann ${ }^{1, *}$, E. Gantois ${ }^{1}$, B. Lagraulet ${ }^{1}$, D. Dautel ${ }^{1}$, E. Cousein ${ }^{1}$, P. Coupé ${ }^{1}$

${ }^{1}$ Pharmacy, Centre Hospitalier de Valenciennes, Valenciennes, France
Background and Objective: Type II heparin-induced thrombocytopenia (HIT) is a known complication of heparin therapy that contraindicates further use of unfractionated heparin or low molecular weight heparin.

In France, two molecules are approved in type II HIT associated thrombosis: danaparoid and argatroban. Because of supply difficulties with danaparoid, our hospital decided to develop argatroban treatment.

Argatroban is a synthetic derivative of L-arginine, a direct and reversible inhibitor of thrombin, manufactured in $250 \mathrm{mg}$ vials. It has to be diluted with $250 \mathrm{~mL}$ of physiological saline. In some features, dosages prescribed are much less, leading to a loss of product if one vial is used for one patient. In order to reduce costs, it was decided to prepare ready-to-use syringes at the pharmacy sterile preparation unit level.

The objective of this study is to assess savings allowed by centralizing the reconstitution of argatroban.

Setting and Method: From October 2011 to April 2013, 22 patients admitted to our hospital were treated with argatroban, 8 in Intensive Care Unit (ICU) and 14 in dialysis.

For each patient, prescriptions and laboratory results (activated partial thromboplastin time (aPTT), platelet count, platelet antibodies) were analyzed.

Results: During 18 months, 1682 syringes were made: 93 in ICU (average 11 per patient) and 1,589 in dialysis (average 113 per patients), representing 799 dialysis sessions.

The target value of aPTT was between 1 and 3 times the initial value. Dosages were adjusted therefore. No treatment was stopped because of an aPTT $>3$.

A total of 172 argatroban vials were used, for a final product cost of 43,000€. Without centralized reconstitution, 857 vials would have been delivered, which would have cost $214,250 €$.

Including the cost of preparation, a saving of $128,005 €$ was achieved, representing almost $50 \%$ of expected expenses.

Conclusions: Argatroban therapy was an efficient and well-tolerated alternative to danaparoid. However, it is an expensive medication and treatment cost may be a limitation for use. Centralized reconstitution of argatroban at the pharmacy is a result of cooperation with the wards. It is a way to ensure preparation quality, and limit treatment cost.

Disclosure of Interest: None Declared.

\section{OCI Hospital pharmacy: clinical education}

\section{HP-CE01}

Why do patients with chronic inflammatory rheumatic diseases discontinue their biologics?

\section{A.-L. Betegnie ${ }^{1, *}$, A. Gauchet ${ }^{2}$, L. Grange ${ }^{3}$, M. Roustit ${ }^{4}$, M. Baudrant ${ }^{1,5}$, P. Bedouch ${ }^{1,5}$, B. Allenet ${ }^{1,5}$}

${ }^{1}$ Pharmacy, University Hospital, ${ }^{2}$ Interuniversity Laboratory of Psychology: Personality, Cognition, Social Change (LIP/PC2S), University, ${ }^{3}$ Rheumatology Clinic, ${ }^{4}$ Center of Clinical Investigation, University Hospital, Grenoble, ${ }^{5}$ CNRS/TIMC-IMAG UMR 5525/Themas, UJF-Grenoble 1, Grenoble F-38041, France

Background and Objective: Adherence is an emerging figure in the field of rheumatology. Results from literature reports adherence rates ranged between 41 and $85 \%$ among patients with chronic inflammatory rheumatic diseases (CIRD) treated with biologics. Various factors may explain this sub-optimal adherence, which can be classified into several dimensions: socio-demographic data, medical and therapeutic history and management of biologic's in daily life. 
The main objective of this study was to explain quantitatively why patients discontinue their biologics by themselves and to identify predictive factors of these self-discontinuations (SD).

Setting and Method: A quantitative and descriptive study was performed using a self-report questionnaire, developed thanks to a preliminary qualitative analysis, which was sent via internet to different patients associations.

Main outcome measures: Exploration of socio-demographic data, level of pain, chronology of the disease and treatments, management of biologic's administration, previous experiences and patient's own resources were requested to explain self-discontinuations (SD). Univariate and multivariate analysis were performed.

Results: A total of 581 patients answered to the questionnaire between 16 June and 4 July 2012, including $60.4 \%$ patients with Ankylosing Spondylitis (351/581), $33.7 \%$ with Rhumatoid Arthritis (196/581), $5.2 \%$ with Psoriatic Arthritis (30/581) and $0.7 \%$ with others CIRD (4/581). Median age was 42 for Asp patients, 55 for RA patients and 50 for PsA patients. More than a thousand lines of biologics were described, with a median of 2 biologics per patients. Eighty-six patients experienced SD (14.8\%).

According to patients, the main reason behind the decision to stop their biologics was the perception of being better (38.4\%). Twentyeight percents reported side effects, $13.4 \%$ reported lack of efficacy, $8.9 \%$ was "fed up" and $7.1 \%$ was afraid about health effects.

Factors which were significantly related to SD in univariate analysis were: biologic's therapy longer than 3 years, sequence of biologic's therapy including more than one line of biologics, self-administered biologics, consumption of alternative medicine, negative beliefs about treatment and lack of medical and social support. Logistic regression analysis also revealed low level of pain as a predictive factor of SD.

Conclusions: These factors should be taken into consideration to better target patients who are more prone to discontinue their biologics by themselves, and to adapt our therapeutic patient education programs.

Disclosure of Interest: None Declared.

\section{HP-CE02}

The influence of medication booklet on adherence rate in type 2 diabetes patients in Bakti Jaya primary health care, Depok, Indonesia

\author{
R. Andrajati ${ }^{1, *}$, A. W. Puspita ${ }^{1}$, A. Bahtiar ${ }^{1}$ \\ ${ }^{1}$ Pharmacology, Pharmacy Faculty, Universitas Indonesia, Depok, \\ Indonesia
}

Background and Objective: To evaluate the influence of a medication booklet on adherence rate using 8-item Morisky scale and glycosylated haemoglobin, in type 2 DM patients.

Setting and Method: Preexperimental prospective study was conducted at Bakti Jaya primary health care, Depok, Indonesia.

Main outcome measures: The score of the 8-item Morisky Medication Adherence Scale (MMAS-8) and percentage of glycosylated haemoglobin $\left(\mathrm{HbA}_{1 \mathrm{C}}\right)$.

Results: A convenience sample of 30 type 2 DM patients was studied to receive medication booklet. The score of the 8-item Morisky Medication Adherence Scale (MMAS-8) and percentage of glycosylated haemoglobin $\left(\mathrm{HbA}_{1 \mathrm{C}}\right)$ were measured before and after the 8 -week intervention. The results were analyzed by paired t-test for $\mathrm{HbA}_{1 \mathrm{C}}$ and Wilcoxon test for MMAS-8. It showed that there were significant differences $(p<0.05)$ of the value of $\mathrm{HbA}_{1 \mathrm{C}}$ and MMAS8 between before and after the 8 -week intervention.

Conclusions: It indicated that diabetes medication booklet, was effective enhancing their medication adherence.

Disclosure of Interest: None Declared.

\section{OCII Hospital pharmacy: pharmaceutical care}

\section{HP-PC01}

Why? A qualitative study of potentially inappropriate prescribing in older patients

S. Cullinan ${ }^{1, *}$, A. Fleming ${ }^{1}$, D. O'Mahony ${ }^{2}$, C. Ryan $^{3}$, D. O'Sullivan' ${ }^{1}$, P. Gallagher ${ }^{2}$, S. Byrne ${ }^{1}$

${ }^{1}$ School of Pharmacy, University College Cork, ${ }^{2}$ Department of Geriatric Medicine, Cork University Hospital, Cork, Ireland, ${ }^{3}$ School of Pharmacy, Queen's University Belfast, Belfast, United Kingdom

Background and Objective: Older patients commonly suffer from multiple conditions and take multiple medications. As a result, these patients are more vulnerable to potentially inappropriate prescribing (PIP). Studies have shown that PIP in these patients results in adverse drug events and hospitalisations (1).

The study aims were: (1) to acquire a deep understanding of doctors' opinions, awareness and experiences of PIP; and (2) to use the findings to inform future interventions which can be targeted to address the areas highlighted.

Setting and Method: Framework analysis. Semi-structured interviews based on a topic guide designed to explore the Theoretical Domains Framework (TDF) (2), with doctors of all grades in four hospitals. Conventional and directed content analysis were used to identify themes and domains that could be targeted in an intervention study. The behaviour change wheel (3) was then used to determine suitable intervention types. This has never been done in the area of PIP.

Main outcome measures: Doctors' opinions as to why PIP occurs, and common themes within these opinions that could be targeted in an intervention study.

Results: 22 doctors were interviewed. Conventional content analysis identified 4 over-arching themes; (1) more education required, (2) environment is conducive to PIP, (3) need for improved IT, (4) lack of collaboration between levels of care. Directed content analysis identified 5 of the 14 domains in the TDF as relevant; (1) environmental context and resources, (2) knowledge, (3) skills, (4) social influences and (5) memory/attention and decision processes. Using the behaviour change wheel, the types of interventions deemed suitable were those based on training and education.

Conclusions: Both methods of content analysis correlated with each other to large extents. Among other reasons for PIP, they strongly indicated that doctors feel there is not enough emphasis on geriatric pharmacotherapy in their undergraduate and post graduate training. We can be confident therefore, that an educational intervention and supplementary training would be justified and beneficial. This study has however, uncovered many areas for potential intervention in the future.

\section{References}

1. Spinewine A, Schmader KE, Barber N, Hughes C, Lapane KL, Swine C, et al. Appropriate prescribing in elderly people: how well can it be measured and optimised? Lancet. 370. England2007. p. 173-84.

2. Michie S, Johnston M, Abraham C, Lawton R, Parker D, Walker A. Making psychological theory useful for implementing evidence based practice: a consensus approach. Qual Saf Health Care. 2005;14(1):26-33.

3. Michie S, van Stralen MM, West R. The behaviour change wheel: a new method for characterising and designing behaviour change interventions. Implement Sci. 2011;6:42.

Disclosure of Interest: None Declared. 


\section{OCII Community pharmacy: pharmaceutical care}

\section{CP-PC01}

Maintaining an annual long-stay prescriptions review: impact on the iatrogenic risk control

G. Leguelinel ${ }^{1, *}$, P. Palassin ${ }^{1}$, L. Di Trapani ${ }^{1}$, J.-M. Kinowski ${ }^{1}$, H. Richard ${ }^{1}$

${ }^{1}$ Pharmacy unit of geriatric centre, University Hospital, Nîmes, France

Background and Objective: Our study aims to assess if maintaining an annual long-stay prescriptions multidisciplinary review has an impact on reducing iatrogenic risk of patients aged $\geq 65$ years.

Setting and Method: The study was conducted on 2 nursing home units (99 residents) and 2 long-stay care units (33 patients). These patients' prescriptions have been reviewed at least once in the past. It's a before-and-after study design.

Main outcome measures: During 15 months, sequential long-stay prescriptions review of patients aged $\geq 65$ years was carried out by a trinomial doctor-pharmacist-nurse. The iatrogenic risk in the elderly is assessed by 2 indicators: the risk score of adverse drug event (ADE) (Trivalle and al, 2013) and the number of patients with at least one potentially inappropriate medication (PIM) according to the Laroche criterias (Laroche et al, 2009).

Results: Our study concerns 127 patients with a mean age of $83.6 \pm 0.7$ years. The average number of drugs prescribed is $9.3 \pm 0.3$ lines per patient. Prescriptions review led to $2.0 \pm 0.1$ pharmaceutical interventions (IP) per patient with $75 \%$ of acceptance, of which $49 \%$ resulted in an interruption of prescription. The average ADE score decreased slightly from 3.0 to 2.8 after review $(p=0.09)$. Similarly, the number of patients with high iatrogenic risk is slightly decreased (14 vs $12 \%)$. The review significantly reduced the percentage of patients with at least one PIM from 16 to $10 \%$ $(p<0.01)$ and the average number of PIM per patient from 1.4 to 0.8 after review $(p<0.05)$.

Conclusions: Our results show that maintaining prescriptions review in the elderly is an effective strategy to improve the quality of therapeutic management by reducing the average number of PIM. The sustainability of the review doesn't seem to be an effective way of influencing the average ADE score, already optimized by previous reviews.

Disclosure of Interest: None Declared.

\section{OCII Hospital pharmacy: pharmaceutical care}

\section{HP-PC02}

Hiv Post-Exposure Prophylaxis Follow-Up Protocol Compliance

L. Villamarín Vallejo ${ }^{1}$, M. Gomis Pastor ${ }^{1, *}$, M. Masip Torné ${ }^{1}$, M. Gutiérrez Macià ${ }^{2}$, G. Mateo Garciá ${ }^{2}$, M. A. Sambeat Domènech ${ }^{2}$, M. A. Mangues Bafalluy ${ }^{1}$

${ }^{1}$ Pharmacy, ${ }^{2}$ Infectious Diseases department, Hospital de la Santa Creu i Sant Pau, Barcelona, Spain

Background and Objective: Post-exposure prophylaxis (PEP) is a short-term antiretroviral treatment to prevent HIV infection after a potential exposure. PEP has a high success rate in adherent patients. However, a previous study conducted in our Institution showed that PEP compliance at follow-up was low. A better clinical follow-up should improve the results of the treatment and therefore have an epidemiological impact.
The aim of this study is to test whether the follow-up compliance of patients receiving PEP improved after the implantation of a dispensation PEP protocol on weekly basis and a multidisciplinary follow-up.

Setting and Method: A retrospective cohort study from January 2011 to December 2012, was performed in a university tertiary hospital in Barcelona. All patients $>18$ years-old starting PEP and with basal HIV antibody test negative were included. Data analyzed included: demographics, type of HIV exposure, time after exposure, side effects.

Main outcome measures: The main outcomes were follow-up compliance (visit and HIV antibody test) at days 1-14, 30, 90 and 180, and seroconversion rates. Data obtained during 2012 (new dispensation PEP protocol on weekly basis) were compared with 2011 data (one-time PEP dispensation).

Results: A total of 85 patients $(2011=40 / 2012=45)$ were included. No significant differences were observed between the groups in terms of age, sex, referral, type of exposure and time to start of PEP.An improvement in compliance with HIV antibody follow-up test (2011 vs 2012): 94.9 vs $100,69.2$ vs $78.3,30.8$ vs 45.7 and 17.9 vs $31.1 \%$ at days $1-14,30,90$ and 180 respectively; and in compliance with the follow-up visits: 76.9 vs $97.8,56.4$ vs $67.4,20.5$ vs 34.8 and 10.3 vs $21.7 \%$ at days $1-14,30,90$ and 180 respectively, was observed. However, no significant differences were found. One seroconversion was detected in the 2012 group at 6 months after the exposure.

Conclusions: The new PEP dispensation protocol on weekly basis and a multidisciplinary follow-up improves the compliance rate at follow-up. However, the percentage of patients that complete the follow-up is still low. In order to exclude seroconversion in all PEP patients further measures are warranted. Disclosure of Interest: None Declared.

\section{HP-PC03}

A follow-up program for patients with established coronary heart disease led by a clinical pharmacist: a randomized controlled trial B. H. Garcia ${ }^{1,2, *}$, T. Giverhaug ${ }^{3}$, J. Utnes ${ }^{2}$, L. Småbrekke ${ }^{2}$

${ }^{1}$ Hospital Pharmacy of North Norway Trust, ${ }^{2}$ Department of Pharmacy, University of Troms $\varnothing,{ }^{3}$ Regional Drug Information Center of North Norway, University Hospital of North Norway, Troms $\varnothing$, Norway

Background and Objective: Major biomedical risk factors for coronary heart disease (CHD) comprise high blood pressure, high blood cholesterol, tobacco use, unhealthy diet, physical inactivity, diabetes, advancing age and genetic disposition. Adherence to therapeutic guideline recommendations have been shown inadequate, and therapeutic targets are frequently not met. Here, we present a 1 year lasting post-discharge follow-up program for patients with established CHD, led by a clinical pharmacist. We aimed to explore how this program influenced adherence to clinical practice guidelines and biomedical risk factors relevant for secondary prevention of CHD.

Setting and Method: Patients were recruited from the Cardiology department at the North Norway University Hospital during the period February 2009-June 2010. We enrolled a total of 102 patients aged 18-82 years into a non-blinded, randomized controlled trial with an intervention group and a control group. The intervention comprised medication reconciliation, medication review and patient education during three follow-up meetings; at discharge, after 3 months and after 12 months. The control group received standard care from their general practitioner. 
Main outcome measures: Primary outcomes were adherence to clinical guidelines recommendations concerning prescription, therapy goal achievement and lifestyle education defined in the medication assessment tool for secondary prevention of CHD (MAT-CHD SP). $_{\text {. }}$. Secondary outcomes included changes the biomedical risk factors blood cholesterol, blood pressure and blood glucose.

Results: Ninety-four patients completed the trial, 48 patients in intervention group and 46 in control group. Appropriate prescribing was high both at baseline and at study end. Therapy goal achievement was low in both groups, and increased slightly from baseline to study end in both groups. However, no significant improvements in biomedical risk factors were observed in favor of the intervention group. Nevertheless, overall adherence to MAT-CHD ${ }_{\mathrm{SP}}$ criteria increased in both groups and was significantly higher in the intervention group at study end compared to the control group, 78.1 vs. $61.4 \%, P<0.001$. The difference was mainly due to an increased documentation of lifestyle advices in intervention group patients.

Conclusions: The clinical pharmacist-led follow-up program significantly increased documented life-style advices defined in the MAT$\mathrm{CHD}_{\mathrm{SP}}$ for the intervention group, but did not lead to significant improvements in biomedical risk factor measures in favor of the intervention group. Even if prescribing of the recommended drugs was high, therapy goal achievement was low in both study groups. Changes to the follow-up program are recommended, the most important being a closer collaboration with the prescriber. In addition, a larger, more adequately powered study should be performed before implementation in standard patient care can be recommended.

Disclosure of Interest: None Declared.

\section{HP-PC04}

Involving community pharmacist to drug adaptation in case of renal impairment: MARIE study

\section{A.-S. Sipert ${ }^{1}$, P. Gatault ${ }^{2}$, N. Hay ${ }^{3}$, J.-M. Halimi ${ }^{2}$, F. Guinard ${ }^{4}$, F. Guegan ${ }^{5}$, X. Pourrat ${ }^{1, *}$ \\ ${ }^{1}$ Pharmacy, CHRU Trousseau, Chambray Les Tours, ${ }^{2}$ nephrology, CHRU Bretonneau, ${ }^{3}$ Pharmacy, Community, Tours, ${ }^{4}$ Biology, Laboratory, Bourges, ${ }^{5}$ Pharmacy, Community, Blois, France}

Background and Objective: Chronic kidney disease (CKD) is defined by an eDFG $<60 \mathrm{~mL} / \mathrm{min}$ during 3 months. The prevalence of CKD is estimated at $10 \%$ in the total population (1). CKD may conduct to drugs interaction and because of pharmacokinetics modifications many drugs eventually need a regimen adaptation to avoid drug side effects and/or increase renal impairment. In France, community pharmacists do not access to lab results of patients; so in case of CKD they can't identify problems. The main objective of this study was to evaluate the impact of drug related problem in case of CKD by given to the pharmacist information on renal status (RS) of patients before delivery.

Setting and Method: prospective and observational study; 24 Community pharmacists $(\mathrm{CP})$ had to select 52 patients $(>65$, diabetes and/or HTA disease) among them those with an $\mathrm{eDFG}<60 \mathrm{~mL} / \mathrm{min}$ (aMDRD calculation, creat $\mathrm{S}$ was transmitted by the laboratory after patient's agreement) or without creat $S$ follow-up were included in the study. For those with $\mathrm{eDFG}<60 \mathrm{~mL} / \mathrm{min}$ prescriptions were analyzed and the eventual problems identified communicated to general practitioners (GP). Before they were trained to analyzed prescriptions in case of CKD by a nephrologist and a hospital clinical pharmacist. A control was done by a team associated a nephrologist and two clinical pharmacists.

Main outcome measures: patients with CKD, patients without renal follow-up, rate of problems identified by $\mathrm{CP}$ and rate of problems resolved.
Results: The data collection was realized during 7 months. 791 patients were pre-included among they, 237 were included (30.05\%): $180 \mathrm{had}$ a GFR $\leq 60 \mathrm{~mL} / \mathrm{min} / 1,73 \mathrm{~m}^{2}$, and 57 hadn't a Creats dosage. The problems rate was $21.5 \%$ and the $\mathrm{CP}$ identification rate was $40 \%$. After transmission to GP one third of problems were resolved. In this study, metformin, allopurinol, fibrate, sitagliptin and statin, were the 5 drugs lively as many problems as prescriptions.

Conclusions: This Preliminary study seemed to show that regarding to the kidney biologics parameters community pharmacists could improve the quality of drug prescription in patients with renal impairment. This study could improved communication between $\mathrm{CP}$ and GP.

Disclosure of Interest: None Declared.

\section{HP-PC05}

Effectiveness of a group-based intervention to improve medication beliefs and adherence in non-adherent patients with rheumatoid arthritis: a randomized controlled trial

H. Zwikker ${ }^{1}$, B. Van Den Bemt ${ }^{2,3, *}$, C. Van den Ende ${ }^{1}$, A. Den Broeder ${ }^{1}$, F. Van den Hoogen ${ }^{1}$, A. Van Dulmen ${ }^{4}$

${ }^{1}$ Department of Rheumatology, ${ }^{2}$ Department of Pharmacy, Sint Maartenskliniek, ${ }^{3}$ Department of Pharmacy, Radboud University Medical Center, Nijmegen, ${ }^{4}$ NIVEL, Utrecht, Netherlands

Background and Objective: Medication adherence in patients with rheumatoid arthritis (RA) is suboptimal, with adherence rates between 30 and $80 \%$. Existing interventions to improve medication adherence are mostly complex and have small effect sizes. We therefore developed a short, group-based intervention for non-adherent RA patients to change the balance in necessity and concern beliefs about medication, and to improve adherence to disease-modifying antirheumatic drugs (DMARDs). This study aims to assess the effectiveness of this intervention on beliefs and adherence.

Setting and Method: This study was conducted in the Sint Maartenskliniek, a hospital specialized in rheumatology, orthopaedics and rehabilitation. Non-adherent (determined with the CQR: Compliance Questionnaire on Rheumatology), adult RA patients using DMARDs were randomized into an intervention or control condition, with an allocation ratio of 1:1. The intervention consisted of two Motivational Interviewing based group sessions led by a pharmacist, a homework assignment, and a follow-up call. Control patients received brochures about their current used DMARDs.

Main outcome measures: The main outcomes were beliefs about medication (assessed with the Beliefs about Medicines Questionnaire) and medication adherence, assessed with the CQR, MARS, and refill rates. Measurements were completed at baseline, and at 1 week, 6 months, and 1 year after the moment of intervention. Generalized Estimating Equations were used to estimate intervention effects.

Results: 123 patients (mean age: 60 years, $69 \%$ female) were randomized. No differences in (the balance between) necessity and concern beliefs about medication and medication non-adherence were detected between the intervention and control condition, except at 12 months follow-up: participants in the intervention condition had less strong necessity beliefs about medication than participants in the control condition (b: -1.0 (95\% CI $-2.0,-0.1)$.

Conclusions: Our group-based intervention neither changed the beliefs about medication, nor improved medication adherence to DMARDs in non-adherent patients with rheumatoid arthritis.

Disclosure of Interest: None Declared. 


\section{HP-PC06}

Assessment of renal function and drug dosage adjustment in hospitalized elderly patients: known but still existing problems

P. Gibert $^{1, *}$, P. Bedouch ${ }^{1,2}$, S. Chanoine ${ }^{1}$, S. Mazière ${ }^{3}$, B. Allenet ${ }^{1,2}$, G. Gavazzi ${ }^{3}$

${ }^{1}$ Pharmacy department, Grenoble University Hospital, ${ }^{2}$ UJF-Grenoble 1/CNRS/TIMC-IMAG UMR 5525/ThEMAS, ${ }^{3}$ Geriatric department, Grenoble University Hospital, Grenoble, France

Background and Objective: Older patients are particularly exposed to adverse drug events. Renal insufficiency in the elderly has to be considered since consequences in drug metabolism and elimination exists. The aim of the study was to report whether Renal Function (RF) was calculated and found in medical records (MR) and whether drug dosages were adjusted to RF in hospitalized patients aged 75 years and more.

Setting and Method: Cross sectional study performed in June 2012 in University Hospital of Grenoble, where Computerized Physician Order Entry system is used. Hospitalized patients aged 75 years and more and present in hospital since at least $24 \mathrm{~h}$.

Main outcome measures: Calculation of RF in MR; drug dosage adjustment to RF.

Results: In this study, 412 patients aged 75 years and more, from 58 units of our hospital, were included and 4,343 drugs were prescribed. Drugs requiring dosage adjustment to RF represented $28.0 \%$ of the prescribed drugs $(n=1,217)$ and were present in the prescription of $97 \%$ patients $(\mathrm{n}=400)$. No calculation of RF was found for $30.2 \%$ patients. The presence of weight $(\mathrm{OR}=55, p<0.0001)$, geriatric $(\mathrm{OR}=17, p<0.0001)$ or clinical pharmacy expertise in the unit $(\mathrm{OR}=37, p<0.0001)$ were associated with a more frequent presence of calculation of RF. When calculation was present, an inappropriate dosage was performed for $30 \%$ of drugs requiring adjustment to RF. Polypathology $(\mathrm{OR}=0.78, p=0.02)$, weight $(\mathrm{OR}=0.98, p=0.03)$ and mild or severe $\mathrm{RF} \quad(\mathrm{OR}=0.04$, $p=0.008$ ) reduce the appropriateness of drug adjustments.

Conclusions: Drug dosage adjustments to RF can be improved. Calculation of RF is fundamental and has to be reported in MR. Pharmacists can play a role in improvement of RF assessment and drug dosage adjustment.

Disclosure of Interest: None Declared.

\section{HP-PC07}

Do my patients receive their pills? Study about medication administration

D. K. Jarraya ${ }^{1, *}$, A. Afroun, A. Simon, O. Benveniste, C. Fernandez, P. Hindlet

${ }^{1}$ Pharmacy department, the pitié-salpêtrière hospital, paris, France

Background and Objective: The aim of the study was to evaluate the quality of drug administration in an Internal Medicine department of a French teaching hospital.

Setting and Method: Drug administration by nurses was assessed in the four units of the department during the medicine administration ( 3 times per day). The assessment lasted for one week in each ward. Data collection and result analysis were performed by 2 senior pharmacists and one resident.

Results: 81 patients (43 females and 38 males, average age of $61 \pm 19$ years old) were included. During the audit period, the administration of 3,465 lines of treatment (101 different drugs) was evaluated. Non-compliance was identified for 280 administrations (8\%) (99 different drugs were concerned). The most frequent non-compliances were omission (65\%), wrong time (15\%) and substitution $(11 \%)$. The absence of patients or drugs was responsible for $57 \%$ and $37 \%$ of the non-compliances respectively. The drug classes mainly concerned were cardiovascular $(18 \%)$, vitamins and oligoelements (16\%), analgesics $(14 \%)$ and GI tract (14\%) drugs. The consecutive repetition of noncompliance for the same drug and for the same patient accounted for $1.3 \%$ of all non-compliances. This repetition is estimated at average of $2.5 \pm 0.1$. Surprisingly, the risk of non-compliance was $18 \%$ higher with medications in the regular stock of the ward than with medicine ordered from the central pharmacy $(p<0.05)$ and $40 \%$ higher for medicines self managed by the patient compared to medicines administrated by nurses $(p<0.05)$.

Conclusions: The rate of administration non-compliance $(8 \%)$ is in agreement with previous published studies (9-18\%). Four main drug classes are associated with more frequent non-compliances. However, a high frequency of non-compliances is not necessarily correlated with a high clinical impact whereas a rare non-compliance could have serious consequences (i.e. omission of desmopressin). Finally, the repeated non-compliances with the same drug for the same patient over time, despite their relative infrequency, have to be taken into consideration and will constitute an improvement target. This study allowed us to point out organizational issues in the drug management system. Therefore, improvement of this system is one of the major targets of the quality approach for clinical pharmacists, nurses and medical staff. Disclosure of Interest: None Declared.

\section{OCIII Pharmacoeconomics}

\section{PEC01}

A fixed budget for drug expenditures can decrease costs while improving quality

C. Artoisenet ${ }^{1}$, J.-D. Hecq ${ }^{1}$, B. Delaere ${ }^{2}$, A. Ghysels ${ }^{2}$, M. Gourdin' ${ }^{2}$, E. Marchand ${ }^{2}$, A. Parent ${ }^{2}$, C. Smulders ${ }^{2}$, A. Spinewine ${ }^{1, *}$

${ }^{1}$ Pharmacy, ${ }^{2}$ CHU UCL Mont-Godinne, Dinant, Yvoir, Belgium

Background and Objective: In 2006 the system for financing drug consumption in Belgian hospitals changed from a fee-for-service to a prospective budgeting system. Each hospital receives a fixed amount of money per hospital stay to cover the expenditures for refundable medicines. This has stimulated our hospital to rationalise the use of drugs.

Objective is to describe initiatives aiming at reducing drug expenditures while maintaining (or improving) quality and implemented from 2010 to 2013 in a 450-bed teaching hospital.

Setting and Method: A multidisciplinary group composed of medical/financial directors, physicians, hospital/clinical pharmacists and analysts regularly discuss initiatives for cost-savings. A part-time clinical pharmacist is responsible for coordination and follow-up.

Opportunities for savings are identified by (a) analysing deviances in expenditures as compared to other Belgian hospitals, for certain diseases and classes of drugs; (b) prospectively auditing prescribing practices.

Main outcome measures: Impact measures include (a) appropriateness of use of drugs and (b) changes in direct costs.

Results: examples of interventions.

Education of HCPs: educational material was developed and regularly communicated to physicians/nurses; information is also available through intranet and the computerized prescribing system. Initiatives focusing on specific (classes of) drugs:

- Sedation practices in ICU were modified to encourage the use of midazolam instead of propofol in specific cases; pre-filled 
syringes of propofol were replaced by vials. Annual costs were reduced by approximately $80.000 €$.

- Procedures for using albumin and colloids were reviewed; the consumption of albumin fell by $80 \%$ over 3 months, without significant increase in the use of cristalloids

- The use of IV bisphosphonates during hospital stay was limited to acute indications (e.g. hypercalcemia)

Initiatives focusing on specific wards: audit and feedback of prescribing practices were performed by a clinical pharmacist on pneumology and otorhinolaryngology wards; the prescription of antibiotics for pneumonia is improving, but no significant change occurred in otorhinolaryngology.

Conclusions: Significant cost savings were achieved, even though some initiatives were unsuccessful and measurement of impact is sometimes challenging. Success requires a structured approach and multidisciplinary collaboration, and involvement of clinical pharmacists is undoubtedly valuable. With the continuous decline of the national budget, further initiatives will have to be implemented. Disclosure of Interest: None Declared.

\section{OCIII Therapeutic drug monitoring and pharmacokinetics}

\section{TDMP01}

Digoxin monitoring program in elderly patients admitted to an Emergency Department

M. Álvarez-Payero ${ }^{1}$, A. Martín-Vila ${ }^{1}$, M. Ucha-Samartín ${ }^{1}$, M. Suárez-Santamaría ${ }^{1, *}$, A. Pichel-Loureiroº, G. Piñeiro-Corrales ${ }^{1}$

${ }^{1}$ Pharmacy, ${ }^{2}$ Emergency Department, University Hospital of Vigo, Vigo, Spain

Background and Objective: Emergency Department(ED) admissions because of Drug Related Problems (DRP) are a well-known fact by health professionals. In our hospital, an interdisciplinary pharmaceutical care program in ED is established, from 2008. A recent retrospective study ${ }^{1}$, in our hospital, shows a rate of $0.34 \%$ for ED admissions by inadequate serum digoxin concentration (SDC), due to overdose mainly. Factors related to digoxin toxicity are: $>80$ years-old, female gender, heart failure, renal failure, drug interactions, narrow therapeutic range, standard dosage in elderly and lack of regular checking of SDC (in community care (CC)). On basis to this study, we established a working group.

The aim of this working group is to implement a digoxin monitoring program, in elderly patients to discharge from the observation room of an ED (OR-ED).

Setting and Method: A systematic literature review (PubMed; pharmacokinetic books) was done for one month. Working group: 2 pharmacists, 3 emergencies physicians and $1 \mathrm{CC}$ physician. Setting: OR-ED, University Hospital. OR have 13 beds.

Main outcome measures: On basis of the information recorded and our cited study, an algorithm was developed to select monitoring candidates. Results: Monitoring program:

(1) Patient treated with digoxin is evaluated by emergency Physician. Algorithm to select candidates was designed:

1. Toxicity suspected;

2. Noncompliance;

3. Elderly patients $(>75$ years-old):

3.1 Digoxin dosage $=250 \mu \mathrm{g}$ daily: SDC extraction and pharmacist monitoring are required.
3.2 Digoxin dosage $<250 \mu \mathrm{g}$ daily: 3.2.1 Creatinine clearance $\geq 60 \mathrm{ml} / \mathrm{min}$ : renal function following. 3.2.2 Creatinine clearance $<60 \mathrm{ml} / \mathrm{min}$ : SDC extraction and pharmacist monitoring are required.

An application form for DSC extraction was elaborated (data: patient identification data, last administration time, extraction time, other medications, diagnosis (atrial fibrillation/congestive cardiac failure).

(2) Digoxin dosage is evaluated by a pharmacist and a dosage recommendation (DR) is notified to ED physicians. Other analyzed data: serum potassium, pharmacodynamic and pharmacokinetic interactions.

(3) Patient is discharged from OR-ED to community: When steady-state serum digoxin concentration is achieved, a new extraction is need and the DR is confirmed or modified by pharmacist. This DR is sent to CC physician.

Conclusions: Digoxin monitoring program is led to elderly ( $>75$ years-old) patient with renal failure or standard dosage admitted to the OR-ED and will be discharged. Algorithm facilitates selection and evaluation of candidate patient. Digoxin monitoring program is being well accepted by ED and CC physicians. Disclosure of Interest: None Declared.

\section{TDMP02}

Carboplatin and weekly paclitaxel doublet chemotherapy in elderly patients with advanced non-small-cell lung cancer (NSCLC): dose reduction and toxicity outcomes

B. Glaser ${ }^{1, *}$, R. Zoubeir ${ }^{1}$, A. Grand ${ }^{1}$, S. Perriat ${ }^{1}$, M. Soussan ${ }^{1}$, J.-M. Canonge ${ }^{1}$, L. Bigay-Game ${ }^{2}$, J. Mazieres ${ }^{2}$

${ }^{1}$ Pharmacy, ${ }^{2}$ Pneumology, CHU Toulouse, Toulouse, France

Background and Objective: A recent phase 3 clinical trial ${ }^{1}$ proved the benefits on morbi-mortality of monthly AUC 6 carboplatin and $90 \mathrm{mg} / \mathrm{m}^{2}$ paclitaxel (days 1,8 and 15), for NSCLC patients aged 70 years and older, despite increased toxic effects. Our study aims at analysing the protocol adaptations due to the age of the patients and the different toxicities observed in practice.

Setting and Method: In a retrospective study from August 2010 to March 2012, we analysed the medical files of every patients treated with this doublet chemotherapy for advanced NSCLC. Monitoring of every chemotherapy cycle, patients' biology and health status evolution has been done.

Main outcome measures: Interval duration, doses administered, days of treatment cancelled and Relative Dose Intensity (RDI) for the treatment. ECOG toxicity scale and WHO performance status score for the patients.

Results: 23 patients have been studied. Their mean age is 74.8 years (95\% CI [70.9; 78.7]), $91 \%$ of them are men. Before treatment, $78 \%$ of the patients have a WHO performance status score between 0 and 1 . After treatment, the mean WHO score rises significantly of 0.3 points $(p<0.005)$. Their creatinine clearance $95 \% \mathrm{CI}$ is $[64.8 ; 79.6] \mathrm{mL} /$ min (Cockroft formula). The mean number of cycles is $3.2(95 \% \mathrm{CI}$ $[2.7 ; 3.7])$ and their interval duration $95 \% \mathrm{CI}$ is $[28.5 ; 30.0]$ days (theoretical interval: 28 days). $56.5 \%$ of patients start with AUC 6 carboplatin and there is a meaningful dose decrease from 1 to 3 AUC points for $26.1 \%$ of the population studied $(p<0.025)$. AUC 6 carboplatin is achieved in only $53.5 \%$ of all cycles compared to $84.2 \%$ in the clinical trial ${ }^{1} .13 .2 \%$ of paclitaxel administrations are cancelled during cycles because of toxicity or infection. The RDI for carboplatin is $83.0 \%$ (95\% CI $[78.2 ; 87.7 \%])$ and $81.9 \%$ for paclitaxel $(95 \%$ CI [75.7\%; $88.1 \%]$ ). About grade 3 and 4 toxicities: 2 patients have worsening general condition, 2 have diarrhoea, nausea or vomiting and 2 have haematological toxicity (neutrophil and platelet). Only 2 have no toxicity but stopped treatment after 1 or 2 cycles. 
Conclusions: Standards are especially deviated by reducing the daily dose of carboplatin even before protocol inclusion. This reduction is not statistically significant because our population studied is way smaller than in the clinical trial. But in practice, we can conclude that the total dose of carboplatin administered per patient is equivalent to an AUC 5 intermediate dose during the whole treatment. Platinum doublet chemotherapy is indicated for fit elderly patients with advanced NSCLC but guidelines tend to be reconsidered for safety reasons.

Disclosure of Interest: None Declared.

\section{OCIII Pharmacoepidemiology}

\section{PE01}

Implementation of risk minimization measures regarding the potentially increased bladder cancer risk with pioglitazone in clinical practice within the UK

\section{J. Spoendlin ${ }^{1,2, *}$, P. Dolin ${ }^{3}$, C. R. Meier ${ }^{1,2}$}

${ }^{1}$ Basel Pharmacoepidemiology Unit, Division of Clinical Pharmacy and Epidemiology, Department of Pharmaceutical Sciences, University of Basel, ${ }^{2}$ Hospital Pharmacy, University Hospital Basel, Basel, Switzerland, ${ }^{3}$ Takeda Development Corporation (Europe), London, United Kingdom

Background and Objective: Pioglitazone is an oral anti-diabetic drug that has been associated with a slight risk increase for bladder cancer. In July 2011, history of or current bladder cancer and uninvestigated macroscopic hematuria were listed as contraindications in the European prescribing information, including an advice on therapy discontinuation with inadequate $\mathrm{HbA} 1_{c}$ control. This study aimed to assess the implementation of these risk minimization measures in clinical practice within the UK.

Setting and Method: We performed a descriptive study using data from the UK-based clinical practice research datalink (CPRD)

Main outcome measures: Among patients with $\geq 1$ pioglitazone prescription between 1999 and 2012, we assessed the proportion of treatment discontinuation in patients with prevalent bladder cancer after the label change. We further analyzed the proportion of pioglitazone treatment cessations upon detection of incident macroscopic hematuria or incident bladder cancer after July 2011, or upon an unfavorable $\mathrm{HbA} 1_{c}$ value, stratified by degree of change in $\mathrm{Hba}_{\mathrm{c}}$ values.

Results: We identified a total of 32,947 pioglitazone users between 1999 and 2012. Pioglitazone therapy was stopped in 41 of 134 current pioglitazone users with prevalent bladder cancer after the label change in July 2011, while 88 patients received $\geq 2$ pioglitazone prescriptions within the year thereafter. Among pioglitazone users after July 2011, 6 of 16 patients with incident bladder cancer and 15 of 67 with incident macroscopic hematuria were withdrawn from the drug. With regard to $\mathrm{HbA1}_{\mathrm{c}}$ control after July 2011, $19.6 \%$ of patients with an $\mathrm{HbA} 1_{c}$ value of 7.5, and $25.2 \%$ of those with a value of $\geq 9 \%$ were taken off the drug immediately. The degree of $\mathrm{HbA} 1_{\mathrm{c}}$ change only marginally affected therapy decisions.

Conclusions: The majority of bladder cancer-risk patients (i.e. incident or prevalent bladder cancer, incident macroscopic hematuria, or inadequate $\mathrm{HbA} 1_{\mathrm{c}}$ response) were not withdrawn from pioglitazone according to the changes of the European prescribing information from July 2011. This study was limited to a short observation period following the label change of the prescribing information. Further follow-up is planned to better quantify compliance.

Disclosure of Interest: None Declared.

\section{OCIII Therapeutic drug monitoring and pharmacokinetics}

\section{TDMP03}

Should anti-infective drugs dosing regimen be adjusted in patients with cystic fibrosis after lung transplantation?

J. Malot ${ }^{1, *}$, C. Chapuis ${ }^{1}$, S. Chanoine ${ }^{1,2}$, E. Honnorat ${ }^{3}$, C. Pison ${ }^{3,4}$, B. Allenet ${ }^{1,5}$, O. Epaulard ${ }^{6}$, B. Camara ${ }^{3}$, P. Bedouch ${ }^{1,5}$

${ }^{1}$ Pharmacy Department, CHU Grenoble, ${ }^{2}$ INSERM/UJF U823, Institut Albert Bonniot, ${ }^{3}$ Pneumology Department, CHU Grenoble, ${ }^{4}$ Inserm U1055, ${ }^{5}$ ThEMAS TIMC-IMAG (UMR CNRS 5525), Joseph Fourier University, Grenoble 1, ${ }^{6}$ Department of Tropical and infectious diseases, CHU Grenoble, Grenoble, France

Background and Objective: Because of pharmacokinetic and pharmacodynamic specificity ${ }^{1}$, anti-infective drugs dosages are much higher in cystic fibrosis than in the general population. The aim of this study was to assess the effect of lung transplantation on anti-infective pharmacokinetics following lung transplantation in patients with cystic fibrosis.

Setting and Method: This analysis was conducted in a 2000-bed University Hospital. The cystic fibrosis patients' files were retrospectively reviewed; these patients had been transplanted between January 2002 and December 2012. We used linear mixed models to compare tobramycin dosage, tobramycin assays, creatinine levels, weight and Body Mass Index (BMI) before and after lung transplantation. Patients identity was used as random variable to account for pseudo replication. $p$ values under 0.05 were considered significant.

Results: Thirty-five patients and 268 anti-infective assays were included. Tobramycin dosages were significantly lower in the posttransplant period $(11.6 \pm 1.8$ vs. $7.9 \pm 1.7 \mathrm{mg} / \mathrm{kg}, \quad p=0.001)$, whereas more trough concentrations of tobramycin were higher than recommended values $(<0.5 \mathrm{mg} / \mathrm{L})$. Creatinine levels significantly increased after transplantation, regardless of patient age, from $63.9 \pm 6.9 \mu \mathrm{mol} / \mathrm{L}$ before transplantation to $104.4 \pm 6.4 \mu \mathrm{mol} / \mathrm{L}$, until one month after transplantation, and to $138.9 \pm 6.6 \mu \mathrm{mol} / \mathrm{L}$ up to one year and more after transplantation $(p<0.0001)$. BMI was also significantly higher after transplantation, increasing from $17.9 \pm 0.4$ to $18.3 \pm 0.4 \mathrm{~kg} / \mathrm{m}^{2}(p=0.01)$.

Conclusions: Renal function is significantly impaired in cystic fibrosis patients after lung transplantation, probably because of longterm immunosuppressive treatment ${ }^{2}$. Excreted in the urine, this impairment could lead to an accumulation of tobramycin; its dosage should therefore be adjusted. A prospective study would allow a more precise evaluation of the effect of lung transplantation on pharmacokinetics of anti-infective drugs, and could lead to an optimization of drug therapy in cystic fibrosis lung transplanted patients.

${ }^{1}$ Horrevorts AM et al. Pharmacokinetics of antimicrobial drugs in cystic fibrosis. Aminoglycoside antibiotics. Chest 1988;94:120S$125 \mathrm{~S}$

${ }^{2} \mathrm{O}$ 'Connell OJ et al. Mechanisms of the rapid decline in glomerular filtration rate following lung transplantation in patients with cystic fibrosis. Am. J. Transplant. 2010;10(4):954

Disclosure of Interest: None Declared.

\section{TDMP04}

Inhibition of cyclosporine and sirolimus metabolism by herbal teas.

S. Dufay ${ }^{1, *}$, A. Monteillier ${ }^{1}$, C. Avanzi ${ }^{1}$, T. F. Ng ${ }^{2}$, J.-M. Garcia ${ }^{3}$, M.-F. Lam ${ }^{4}$, I. C. K. Wong' ${ }^{2}$, A. Worsley ${ }^{2}$ 
${ }^{1}$ Department of Pharmacology and Pharmacy, The University of Hong Kong, ${ }^{2}$ Department of Pharmacology and Pharmacy, The University of Hong Kong, Hong Kong, Hong Kong, ${ }^{3}$ Institut Pasteur Korea, Seoul, Korea, Republic Of, ${ }^{4}$ Queen Mary Hospital, Hong Kong, Hong Kong

Background and Objective: Cyclosporine and sirolimus, immunosuppressive treatments with narrow therapeutic window, are mainly metabolized by cytochrome 3A4 (CYP3A4) in the liver. A clinical case of interaction after consumption of a "24-flavours" tea, popular herbal beverage in China, leading to an increase of cyclosporine and sirolimus blood concentrations to toxic levels was reported. This study aimed to identify the causative ingredients of herbal tea in the inhibition of CYP3A4 metabolism.

Setting and Method: An in vitro assay involving human CYP3A4 was developed. Different "24-flavour" teas and six of their common plant constituents were tested for their inhibitory effect on CYP3A4. Recommended herbal tea or plants' posology were used to design the study.

Main outcome measures: Measure of the concentration of herbal tea and 6 most used plants extract that inhibits fifty percent of cytochrome P450 3A4 (IC50 \%).

Results: Two brands of "24-flavours" tea and the 6 plants showed inhibition activity at therapeutic doses. The 24 -flavour tea drunk by the patient is the most potent $\left(\mathrm{IC}_{50}=190.9 \mu \mathrm{g} / \mathrm{mL}\right)$. The most potent plant is Chrysanthemum with an $\mathrm{IC}_{50}$ of $95.7 \mu \mathrm{g} / \mathrm{mL}$. The three second potent plants are Dandelion (Taraxacum momgolicum), Liquorice (Glycyrrhiza ularensis) and Bishop's weed (Houttuynia cordata) with $\mathrm{IC}_{50}$ of $140.6,148.4$ and $185.5 \mu \mathrm{g} / \mathrm{mL}$ respectively. Peppermint (Mentha haplocalyx) and Japanese Honeysuckle (Lonicera japonica) have weaker inhibitory effect on CYP3A4 with $\mathrm{IC}_{50}$ of 1153.3 and $1,466.3 \mu \mathrm{g} / \mathrm{mL}$.

Conclusions: This study confirms the possible implication of wild spread herbal tea constituents in the inhibition of cyclosporin and sirolimus' metabolism by CYP3A4. It is suggested that concurrent use of herbal teas containing one of those constituents with cyclosporine and/or sirolimus or other medications whose metabolism involves CYP3A4 require close monitoring.

Disclosure of Interest: None Declared.

\section{TDMP05}

Isoniazid toxicity with therapeutic dose in a patient with pulmonary tuberculosis: a case report

L. Sylvestre ${ }^{1}$, A. C. Gairard-Dory ${ }^{1}$, A. Molard ${ }^{2}$, N. Khayath ${ }^{2}$, D. Leveque ${ }^{1, *}$, B. Gourieux ${ }^{1}$

${ }^{1}$ Pharmacy-Sterilization, ${ }^{2}$ Pneumology, University Hospital, Strasbourg, France

Background and Objective: Isoniazid (INH) is a key drug for tuberculosis treatment which is eliminated through metabolization by the genetically polymorphic $N$-acetyltransferase 2 (NAT-2). The rate of elimination of INH is trimodally distributed with rapid/intermediate and slow acetylators. Sixty percent of black African or Caucasian populations and ten percent of Asiatic populations are slow acetylators.

Setting and Method: A Senegalese woman of $70 \mathrm{~kg}$ presented weight loss, fever, productive cough and dyspnea. The diagnosis of pulmonary tuberculosis was made with the presence of BAAR in sputum and with lobar opacity on chest radiography. The treatment was initiated by isoniazid, rifampicin, ethambutol and pyrazinamide. The initial dosage of isoniazid was $5 \mathrm{mg} / \mathrm{kg} /$ day ( $350 \mathrm{mg} /$ day).

Main outcome measures: Serum isoniazid concentration was measured $3 \mathrm{~h}$ after oral administration.
Results: Four days after the beginning of the treatment, serum INH concentration was $6.90 \mathrm{mg} / \mathrm{L} 3 \mathrm{~h}$ after oral administration (normal range $1-2 \mathrm{mg} / \mathrm{L}$ ). The dosage was reduced to $100 \mathrm{mg} /$ day which led to INH concentration of $1.40 \mathrm{mg} / \mathrm{L}$. Two months later, the quadritherapy was switched to rifinah ${ }^{\circledR}$ (INH: $150 \mathrm{mg}$; rifampin: $300 \mathrm{mg}$ ) two pills a day. One week later, she presented nausea, vomiting, deterioration of the general status and was admitted to the hospital. The INH serum concentration was $6.43 \mathrm{mg} / \mathrm{L}$. The dosage of INH was reduced again to $100 \mathrm{mg} /$ day. The symptoms partially resolved and the patient was discharged from the hospital and the treatment was continuated for 4 months.

Conclusions: First side effects were in relation with high INH serum levels. This overdosage may be explained by the slow acetylation profile of this patient. There are no current guidelines to propose systematic NAT2 genotyping before INH administration but therapeutic monitoring is mandatory to adjust dosing.

Disclosure of Interest: None Declared.

\section{OCIII Pharmacoeconomics}

\section{PEC02}

Impact of prescriptions review on the quality and cost of nursing home residents' therapeutic management

G. Leguelinel1 ${ }^{1}$, L. Di Trapani ${ }^{1}$, J. Rolain ${ }^{1}$, J.-M. Kinowski ${ }^{1}$, H. Richard ${ }^{1, *}$

${ }^{1}$ Pharmacy unit of geriatric centre, University Hospital, Nîmes, France

Background and Objective: Our study aims to assess the impact of a prescriptions multidisciplinary review on the quality and cost of therapeutic management of patients aged $\geq 65$ years in a nursing home.

Setting and Method: The study was conducted on two nursing home units: one unit as a control group (group 1) and one unit benefiting from prescriptions review (group 2). It was designed as a costeffectiveness analysis with two phases: observational phase (phase 1), and interventional phase with prescription review (phase 2).

Main outcome measures: In phase 1, 3 indicators were collected for each patient: the risk score of adverse drug event (ADE) (Trivalle and al, 2,013), the number of potentially inappropriate medications (PIM) (Laroche and al, 2009), drug and dedicated medical time costs. Actual costs were calculated from the local rate base per patient per day and then extrapolated to the 500 residents nursing home for 1 year. In phase 2, the same indicators are calculated 1 month later.

Results: 48 patients were included in group 1 and 46 patients in group 2. The average risk score of ADE decreased from $2.3 \pm 0.4$ to $1.8 \pm 0.3$ in group 2 , while it remained at $2.8 \pm 0.3$ in group 1 . The distribution between the levels of risk score of $\mathrm{ADE}$, initially homogeneous in the two groups, was significantly different in group 2 , before versus after review $(p<0.005)$. The number of patients in group 2 with a high risk score of $\mathrm{ADE}(11 \%)$ is divided by five and those with at least one PIM (17\%) is divided by 4 after review. The difference between the average drug cost before and after review would be estimated at $24,000 \pm 7,500 €$ a year for all patients of the nursing home (500 patients).

Conclusions: Prescriptions multidisciplinary review is a cost-effective strategy for our 500 residents' nursing home with an estimated net gain of $13,000 € /$ year and a decrease of -0.5 risk score of ADE after review. The impact of prescriptions review is probably underestimated due to other indirect costs avoided and difficult to estimate (exhaustive collection of $\mathrm{ADE}$ and their criticality, number of emergency hospitalization avoided).

Disclosure of Interest: None Declared. 


\section{PDFI Hospital pharmacy: pharmaceutical care}

\section{HP-PC08}

Development of computerized prescription: "pharmacists, down to work!"

P. Calvet ${ }^{1, *}$, L. Damery ${ }^{1}$, A. Cyrus ${ }^{1}$, J.-J. Tiravy ${ }^{1}$, C. Rouzaud-Laborde ${ }^{1,2,3}$

${ }^{1}$ Pharmacy pole, Toulouse University Hospital, ${ }^{2}$ Unit 1048 team 6, INSERM I2MC, ${ }^{3}$ Clinical Pharmacist Teacher, Paul Sabatier University, Toulouse, France

Background and Objective: Computerized prescription order entry (CPOE) is developing in France and contributes to the decrease of medication errors. However, computerization might generate new mistakes. The main objective of this work is to determine pharmaceutical interventions (PIs) due to new computerization project, moreover PIs evolution month by month.

Setting and Method: We relate error types according to French Society of Clinical Pharmacy's classification during the computerization of the neurosciences department in neurovascular care unit (26 hospital beds), at Toulouse University Hospital. For 6 months, all prescriptions in Orbis ${ }^{\circledR}$ software were reviewed for interactive analysis by pharmacists. Three pharmacists (two senior pharmacists and one resident) performed these validations. A comparison of the proportions was realized according to the Fisher exact test.

Results: Over the 6 months, 638 PIs were performed. $13.3 \%$ were errors generated by the computer use. Among others PIs, $35.1 \%$ concerned unadapted way or administration of medications, $18 \%$ non conformed recommendations or contraindications, $9.7 \%$ overdoses, $4.6 \%$ under dosages, $4.4 \%$ inappropriate treatments, $4 \%$ untreated indication, $3.2 \%$ adverse effects, $3 \%$ monitorings, $2.7 \%$ unreceipt treatments and $2.1 \%$ interactions. $70 \%$ of PI were accepted.

Among PIs due to CPOE errors, we reported duplicate orders, unit errors, the use of free text, times errors and parameterization flaws. We also observed a decrease in the proportion of the PIs from November to April. The interventions of the pharmacists due to CPOE errors were significantly differents according to the months: $28.6 \%$ of the totality of the PIs in November, $22 \%$ in December, $13.8 \%$ in January, $20.9 \%$ in February, $15.5 \%$ in March and $6.3 \%$ in April $(p=0.0089)$. An augmentation was observed in February $(20.9 \%)$. This phenomenon is due to the switch of physician resident in care unit.

Conclusions: This work shows that the pharmacists have to be careful at the beginning of a computerized project. After three months, the rate CPOE errors in our study is acceptable. Apart from this critical period, this tool makes data more accessible for pharmacist. As a result, the pharmaceutical team can be more determined in prescription analysis and on individual information given to the patient.

Disclosure of Interest: None Declared.

\section{HP-PC09}

Off-Label Use of "High-cost" drugs in Paris area hospitals

S. Kabiche ${ }^{1, *}$, D. Monzat ${ }^{1}$, M. Le Jouan ${ }^{1}$, R. Farinotti ${ }^{1}$, C. Montagnier-Petrissans ${ }^{2}$, P. Le Gonidec ${ }^{1}$

${ }^{1}$ Observatory of Drugs, Medical Devices and Therapeutic Innovations (OMEDIT), ILE De France, Regional Health Agency (ARS), ILE De France, ${ }^{2}$ Medical and Administrative Directorate (DMA), AP-HP, Paris, France
Background and Objective: In 2012, In Paris area hospitals, total spending in "High-cost" drugs amounted to $€ 473$ million. Off-Label Use (OLU) of these drugs is relatively common in medical practice, even if it's often not supported by strong scientific evidence. The Observatory of Drugs, Medical Devices and Therapeutic Innovations (OMEDIT), in its monitoring and analysis of prescribing practices is a player in control of drug spending trends. The aim of this study was to assess the prescriptions' adherence to the good practice guidelines for "High-cost" drugs, identifying OLU situations emerging that could lead to an update of the standards.

Setting and Method: The data were collected by OMEDIT, from 218 Paris area hospitals over a period of six months (January to June 2012). For each OLU situation recorded, the following data were collected: drug name, therapeutic indication, clinical justification, literature justification, frequency (number of patients affected).

Main outcome measures: Rate of patients involved in Off-Label "High-cost" drug use.

Results: 1499 OLU situations were reported, they concerned 3,267 patients. The OLU of anticancer drugs affected $53 \%$ of patients. The anticancer drugs mostly involved in these situations were: Bevacizumab, Rituximab, Trastuzumab, Bortezomib and Pemetrexed in respectively $34,25,12,11$ and $7 \%$ of patients concerned. For other clinical areas, five drugs were prescribed on $84 \%$ of patients involved in the OLU situations: Human Prothrombin Complex, Intravenous Immunoglobulin (IVIG), Rituximab, Caspofungin and Infliximab in respectively $25,21,18,12$ and $8 \%$ of patients concerned. The OLU situations mainly described were Rituximab in lymphoma (regarding to stage and/or line of therapy) and the use of Human Prothrombin Complex for Hæmostasis Disorders in Open Heart Surgery with Extracorporeal Circulation.

Conclusions: The most prescribed drugs were the most involved in OLU situations. For some indications, benefit-risk assessments for real life prescribing practices should be set up in order to collect scientific information to ensure a safe use. In France, Temporary Recommendations for Use (TRUs) can be an answer for regulating Off-Label drug Use.

Disclosure of Interest: None Declared.

\section{HP-PC10}

Establishing a geriatric interprofessional out-patient clinic for medication review

K. K. Viktil ${ }^{1,2, *}$, E. Engh ${ }^{3}$, H. Frøyland ${ }^{1}$, A. H. Ranhoff ${ }^{3}$

${ }^{1}$ Diakonhjemmet Hospital Pharmacy, ${ }^{2}$ School of Pharmacy, University of Oslo, ${ }^{3}$ Department of Medicine, Diakonhjemmet Hospital, Oslo, Norway

Background and Objective: Comorbidities and hence long medication lists and drug-related problems in elderly patients are important, and contribute to morbidity, hospital admission and mortality. It is well known that during a short hospital stay or during a visit to their general practitioner or at the pharmacy it is a challenge to take care of all aspects of the medications. We therefore aimed to develop an out-patient clinic for these patients with special focus on medication review.

Setting and Method: Hospital, department of medicine, section geriatric out-patient clinic. The clinic should be inter-professional with geriatricians, nurses and clinical pharmacists. Further, the clinic should be based on the concordance principles and the dialogue with the patient is essential.

Main outcome measures: Establishing the logistics and framework for the out-patient clinic. 
Results: Patients are referred from hospital physicians and general practitioners. The patient is actively involved in a) assessing function in daily life activities (nurse), b) a systematic evaluation of the patient's use of medications (clinical pharmacist), and a medical clinical evaluation including vital and other signs and symptoms (geriatrician). These three steps are undertaken at the same day, and immediately thereafter a consensus meeting with all involved health professionals and the patient him-/herself is held to discuss the drugrelated problems identified and also to prioritize and agree upon solutions. The geriatrician writes an inter-professional summary, which is send to the patient's general practitioner. The 10 first patients ( 8 women/2 men), mean age 81.7 years (range 76-88), used a mean of 12.7 drugs at admission (range 1-26) of which 16 drugs (range 1-3) were classified as a STOPP drug. It was identified 5.5 drugrelated problems/patient (range 1-11). The medication review resulted in 3.8 changes/patient in their drug regimen (range 0-8).

Experiences from setting up the out-patient clinical have resulted in a chapter, "Medication Review", in the Norwegian Guideline of Medications for Health Professionals.

Conclusions: A full medication review of complex elderly patients includes assessments from nurse, clinical pharmacist and geriatrician. The perspective of the patient is essential to optimize the outcome and to reduce the risk of medications.

Disclosure of Interest: None Declared.

\section{HP-PC11}

Venous thromboembolism prophylaxis among acute medical patients in an emergency department

\section{Jolly ${ }^{1, *}$, F. Raffy ${ }^{1}$, P. Atain-Kouadio ${ }^{2}$, A. Valance ${ }^{2}$, L. Nace ${ }^{2}$ \\ ${ }^{1}$ Pharmacy, ${ }^{2}$ Emergency department, University Hospital, Nancy, France}

Background and Objective: This study aims to determine the proportion of medical patient hospitalised in an emergency department receiving thromboprophylaxis, to evaluate the adequacy of such prescription as well as its success rate.

Setting and Method: The indication for and adequacy of thromboprophylaxis were evaluated by the French guidelines of ANSM on Venous Thromboembolism Disease in acutely medical condition.

A retrospective study in three periods of one month was used, each which 50 medical records selected. These medical records were evaluated (G1) before an effective reminder of the guidelines (G2). Reevaluation after 4 months (G3) to gauge the progress was also performed.

Were taken into account: demographic, biological, clinical data and the relevant informations on the use of heparine and fondaparinux were recorded.

Analysis of variance, Fisher's exact test and Chi square test were used for values of $p<0.05$ considered significant ones.

Results: Age, Body Mass Index, MDRD were not significantly different in the three groups $(p<0.05)$. Thromboprophylaxis was indicated in $68 \%$ for G1 vs $62 \%$ for G2 vs $60 \%$ for G3 ( $p=0.68)$. $20 \%$ of this study's patients received thromboprophylaxis for G1 vs $58 \%$ for G2 vs $20 \%$ for G3 $(p=0.001)$. When pharmacologic prophylaxis was prescribed, we observed an increase of the appropriateness of the use of thromboprophylaxis regarding: drug and dosing regimen ( $42 \%$ in G1 vs $88 \%$ in G2 $(p=0.03)$ vs $100 \%$ in $\mathrm{G} 3(p>0.05))$, administration within the time limit $(71 \%$ for $\mathrm{G} 1 \mathrm{vs}$ $94 \%$ for G2 $(p>0.05)$ vs $100 \%$ for G3 $(p>0.05))$, biological check-up before administration (43\% for G1 vs $94 \%$ for G2 $(p=0.012)$ vs $100 \% \mathrm{G} 3(p>0.05))$ and biological follow-up during the treatment (43\% for G1 vs $100 \%$ for G2 and G3 $(p=0.003)$ ).

Conclusions: The increase of the rate and adequacy of the thromboprophylaxis between $\mathrm{G} 1$ and G2 demonstrates the impact of a reminder of these guidelines to physicians. The fact thromboprophylaxis's prescriptions decreases between G2 and G3 can be explained by the guideline's absence of diffusion in particular to the new residents. These results justify and prove the need, to strengthen training and operate regular reminders on thromboprophylaxis. Disclosure of Interest: None Declared.

\section{HP-PC12}

Impact of clinical pharmacist in a pediatric medecine department G. Kosmalski ${ }^{1}$, A.-C. Gerout ${ }^{1}$, M. Fischbach ${ }^{2}$, B. Gourieux ${ }^{1}{ }^{*}$

${ }^{1}$ Service de Pharmacie-Stérilisation, ${ }^{2}$ Service de médecine pédiatrique, Hôpitaux Universitaires de Strasbourg, 1 avenue Molière, 67098 Strasbourg, France

Background and Objective: Evaluate the impact of the presence of a clinical pharmacist in the pediatric medicine department by the evaluation of pharmaceutical interventions.

Setting and Method: An analysis of therapeutical prescriptions was conducted (every 2 days for every child) by a clinical pharmacist in the pediatric medicine department ( 38 beds) for a period of 6 months. With a tool coding our clinical pharmacy interventions, developed by the working group of the French Society of Clinical Pharmacy, we were able to measure several indicators: type and frequency of detected drug related problems, type of pharmaceutical interventions and acceptance by physicians.

Results: Between November 5 and April 30 2013, 1,706 prescriptions were analyzed and generated 455 pharmaceutical interventions (27\% of prescriptions, $5 \%$ of the prescribed drugs). We proposed 136 pediatric formulations. The interventions especially concerned the drugs of alimentary tract and metabolism (26\%), anti-infectious $(20 \%)$ and nervous system drugs $(17 \%)$. Interventions were mainly accepted $(77 \%)$ by doctors. The drug related problems included: optimization of methods of administration (24\%), non compliance with standards $(21.5 \%)$, overdose $(12 \%)$, drug not indicated $(11 \%)$, drug interactions $(8 \%)$, untreated indication $(7 \%)$, a monitoring procedure $(6.5 \%)$, under-dosing $(6 \%)$, sides effects $(3.5 \%)$, treatment not received $(0.5 \%)$. Pharmaceuticals interventions led to a dose adjustment (32\%), therapeutic monitoring (24\%), substitution $(12 \%)$, discontinuation of therapy $(11 \%)$, optimization of administration modalities (11\%), addition of a drug (5\%) and choice of the route of administration $(5 \%)$. We declared seven statements of pharmacovigilance.

Conclusions: The presence of a clinical pharmacist in the pediatric department is necessary and contributes to optimize the drugs care for these patients at risk. The main pharmaceutical intervention involved optimization of the administration. Last but not least, we should measure the clinical relevance of our pharmaceutical activity.

Disclosure of Interest: None Declared.

\section{PDFI Hospital pharmacy: clinical education}

\section{HP-PC13}

Off-label use of psychotropic medications in paediatric oncology: a prospective study

\author{
J. Giraud ${ }^{1, *}$, K. Morand ${ }^{1}$, A. Fratta ${ }^{1}$, G. Benoit ${ }^{1}$, G. Leverger ${ }^{2}$
}

${ }^{1}$ Pharmacy, ${ }^{2}$ Oncology Paediatric, Armand-Trousseau, Paris, France

Background and Objective: In paediatric population, many drugs are off-label used because of the narrowness of the market and the 
lack of clinical trials. Psychotropic drugs are largely concerned, when psychotherapy is not efficient.

Children with malignant diseases (leukemias, solid tumors) are susceptible to experiment an aggravation of their psychiatric symptoms at the announcement or during hospitalizations. In this ward psychotropic medications are often prescribed.

To improve professional practice and optimize the paediatric used of psychotropic medications a study was conducted in our oncology department.

Setting and Method: A prospective analysis of all psychotropscontaining prescriptions was realized every day during 4 weeks. Datas were recorded from informatic prescriptions and medical files, with medical team cooperation (age, indications, dosage...). Datas were reported and analyzed with respect to licence status of drugs and reasons for off-label prescriptions.

Results: On 78 analysed prescriptions, 20 children have received one or more psychotropic medications (five different drugs were listed). Sixty three percent of prescriptions were off-label $(90 \%$ due to indication, $10 \%$ for age), but $79 \%$ of them are documented in literature (Paediatrics dosage handbook). The most prescribed drugs were anxiolytic $(40 \%)$ and antidepressant $(27 \%)$ whereas only $40 \%$ of children suffered from an anxious or depressive disorder. In the same way, antipsychotic drugs represent $20 \%$ of prescriptions whereas 2 children have a behavioural disorder. We find that two third of off-label drugs (clonazepam, chlorpromazine) are used for chemotherapy side effects (neuropathic pain, vomiting).

Conclusions: Our rate of off-label prescriptions is very important but it is similar to other studies (66\% in Robert Debré hospital). We can noticed that $52 \%$ of off-label prescriptions is often used in oncology paediatric practice to prevent side effects.

Unpublished cases concern $20 \%$ of off-label prescriptions, and exclusively for psychiatric indications. Thus the therapeutic strategy seems to be problematic. In order to optimize the use of psychotropic drugs in our oncology ward, pharmacists have proposed a decisional table for psychotropic medications prescribing in psychiatric indication.

Disclosure of Interest: None Declared.

\section{PDFI Hospital pharmacy: pharmaceutical care}

\section{HP-PC15}

\section{Clinical pharmacy services in a neurosurgery ward}

\section{A. Jarfaut ${ }^{1}$, Y. Nivoix ${ }^{1}$, D. Vigouroux ${ }^{2}$, J. Gaudias ${ }^{3}$, D. Leveque ${ }^{1, *}$,} B. Gourieux ${ }^{1}$

${ }^{1}$ Pharmacy, ${ }^{2}$ Neurosurgery, ${ }^{3}$ Orthopaedic Surgery, Hôpitaux Universitaires de Strasbourg, Strasbourg, France

Background and Objective: Clinical pharmacists contribute to the safe medication use. Little is known regarding their impact in surgery. The purpose of this prospective study was to document and evaluate the services of clinical pharmacists in a neurosurgery ward.

Setting and Method: Clinical pharmacy services (medication review and reconciliation, participation on rounds) were prospectively collected between may and August 2012 in a 56-bed neurosurgery ward. Medication problems, interventions, acceptance rate and time for each service were recorded by 2 clinical pharmacists. Clinical impact of interventions was retrospectively assessed by 2 pharmacists and 2 physicians according to Hatoum's scale (extremely significant, very significant, significant and no significant).

Main outcome measures: Medication problems, type and clinical significance of intervention, acceptance rate, daily time for clinical pharmacy services.

Results: During the study period, 1135 prescriptions were analyzed. The pharmacists identified 85 drug-related problems $(7.5 \%$ of the prescriptions) primarily related to gastro-intestinal drugs (27/85) and anti-infective agents $(19 / 85)$. Medication problems $(\mathrm{n}=85)$ mostly included drug omissions (36\%), underdoing $(11.7 \%)$ and inappropriate medications $(10.5 \%)$. Interventions $(n=85)$ led to drug additions (47\%), drug discontinuations (20\%) and replacement of a drug by another one $(11.8 \%)$. Most $(96.5 \%)$ of the interventions were accepted by the physicians. Regarding the potential clinical impact, $50.6 \%$ were judged significant including $8.2 \%$ very significant. No intervention was estimated extremely significant. Mean daily time for clinical pharmacy services were $3 \mathrm{~h} 50 \mathrm{~min}$ including $19 \mathrm{~min}$ for 2.6 reconciliations, $121 \mathrm{~min}$ for 20 medication reviews, $83 \mathrm{~min}$ for round participation and $33 \mathrm{~min}$ for data recording.

Conclusions: The integration of clinical pharmacy services resulted in drug specific interventions in $7.5 \%$ of the prescriptions of patients hospitalized in neurosurgery. Half of the interventions were judged clinically significant. The intervention acceptance rate by physicians was high.

Disclosure of Interest: None Declared.

\section{HP-PC16}

Drug shortages in retail pharmacies: which economic impact, management difficulties and challenges for hospital pharmacies?

C. Boczek ${ }^{1, *}$, C. Vigier $^{1}$, C. Jacob ${ }^{1}$, S. Dumas ${ }^{1}$

${ }^{1}$ Service Pharmacie, CHU Nice Hospital Pasteur, NICE, France

Background and Objective: In recent years, the amount of shortages has grown continuously particularly with drugs usually dispensed in retail pharmacies. To ensure continuity of treatment, hospital pharmacies have to deliver imported alternative drugs or requiring temporary authorisation order of the ANSM (Medicines Control Agency).

The main objective of this study is to measure the evolution of the activity generated by these shortages and evaluate their economic impact.

Setting and Method: In July 01st 2013, an inventory of drugs normally dispensed in retail pharmacies, out of stock over the past year (2012/2013), using the website of the ANSM, was carried out.

A retrospective analysis of the number and nature of services provided because of drug shortages has been performed in our hospital (from 1st January 2012 to 30th June 2013), via our pharmaceutical management and dispensing software.

Main outcome measures: Identify and determine the nature and amount of drugs involved, the number of patients, the duration of shortages, the extra cost to the hospital.

Results: Our hospital pharmacy was concerned by the shortage of 7 products usually in the community: phenytoin, epinephrine, fludrocortisone acetate, potassium perchlorate, iproniazid, propylthiouracyl, monopotassium phosphate.

1,031 dispensings were due to drugs' shortages ( $28 \%$ of all dispensings), total of $€ 18373$ with mainly substitution drug of phenytoin (€6667; 828 dispensings), followed by substitution drug of fludrocortisone acetate ( $€ 5624 ; 19$ dispensings) and substitution drug of propylthiouracyl (€3210; 142 dispensings).

Each prescription (excluding drug costs) generates management fees of $€ 22$ and overall amounted to a total of $€ 22,682$.

264 patients were involved. The average duration of each shortage was 11.7 months.

Each dispensing takes 15 min on average, and these exceptions have totalled $258 \mathrm{~h}$ of pharmaceutical staff time (0.10PST).

Conclusions: Management of shortages in retail pharmacies makes up for an increasing amount hospital pharmacy activity and resources. Indeed, the staff must spend time managing orders (phone calls from patients, pharmacists and laboratories), the spread of information (patients, physicians, city pharmacists). 
The time dedicated to the activity of retrocession has increased: information on the shortage (cause, time), new storage conditions, terms of use and dose adjustment.

It would be interesting to work on guidelines on managing drug product shortages retail pharmacies and their impact to hospital, and too, drug shortages in hospitals and their impact on health systems. Disclosure of Interest: None Declared.

\section{HP-PC17}

POPI: a tool to identify inappropriate prescribing in pediatrics. Consensus validation

T. Weil ${ }^{1}$, F. Angoulvant ${ }^{2}$, R. Boulkedid ${ }^{3}$, C. Alberti $^{3}$, F. Brion ${ }^{4}$, O. Bourdon ${ }^{4, *}$, S. Prot-Labarthe ${ }^{5}$

${ }^{1}$ Pharmacy, ${ }^{2}$ Emergency Department, ${ }^{3}$ Clinical Research Unit, APHP Hôpital Robert Debré, ${ }^{4}$ Pharmacy, APHP Hôpital Robert Debré Université Paris Descartes Pharmacie Clinique, Laboratoire Education et Pratiques de Santé Université Paris XIII Bobigny, ${ }^{5}$ Phamacy, APHP Hôpital Robert Debré, Paris, France

Background and Objective: To develop a tool to identify omission and inappropriate prescription in pediatrics based on French and international guidelines. Validation by consensus method (Delphi process).

Setting and Method: Literature review; design and validate a tool called POPI (Omission and Inappropriate Prescriptions in Pediatrics) using a Delphi method.

Main outcome measures: Obtain a list of inappropriate prescriptions and omission to develop a tool, based on evidence-based medicine, which aims to reduce prescribing error and adverse events in pediatrics.

Results: 108 propositions (80 inappropriate prescriptions and 28 omissions) were obtained by literature review and submitted to a 16-member expert panel ( 8 pharmacists, 8 physicians hospital-based-50\%-or working in community-50\%). These propositions were categorized according to the main physiological systems (gastroenterology, respiratory infections, pain, neurology, dermatology and miscellaneous). Each criterion was accompanied by a concise explanation as to why the practice is potentially inappropriate in pediatrics (included references). Two rounds of the Delphi process were completed by a survey website. 89 out 93 first propositions submitted to expert were selected after 2 Delphi rounds (66 inappropriate prescriptions, and 23 omissions). Validation of gastroenterology criteria are still in progress (15 criteria).

Conclusions: POPI is the first tool developed to detect inappropriate prescription and omission in pediatrics based on explicit criteria. Inter-rater reliability study is necessary before using the tool and prospective study to assess the effectiveness of POPI. We hope POPI to be a valid, reliable and comprehensive screening tool that enables the prescribing physician and clinical pharmacists to evaluate patient's prescription drugs in the context of one current diagnosis. Disclosure of Interest: None Declared.

\section{HP-PC18}

\section{Pharmacist intervention in pain management following heart} surgery

D. Agius Decelis ${ }^{1}$, M. Zarb Adami ${ }^{1}$, J. Galea ${ }^{2}$, A. Serracino Inglott ${ }^{1, *}$, L. M. Azzopardi ${ }^{1}$

${ }^{1}$ Department of Pharmacy, Faculty of Medicine and Surgery, ${ }^{2}$ Department of Surgery, Faculty of Medicine and Surgery, University of Malta, Msida, Malta
Background and Objective: Pain is a common complaint following cardiac surgery and its proper management is imperative to reduce the risk of cardiovascular complications. Postoperative regular oral paracetamol has been shown to be an effective analgesic after discharge from hospital.

The objective is to study the influence of pharmacist intervention in preventing and relieving pain in cardiac surgical patients.

Setting and Method: Following University Research Ethics Committee approval, 115 consecutive post-cardiac surgery patients were recruited at the Cardiac Surgical Ward, Mater Dei Hospital, Malta. Patients were divided into intervention (A) and control (B) groups depending on whether the last digit of the hospital number was odd or even. Group A patients were given a booklet that included the consent form, information about pain, pictorial diagrams on how to help decrease and avoid pain, an analgesia dosage regimen scheme and a diary to annotate daily periodic medication intake along with pain scoring charts. Group B patients were given the consent form, the diary and the pain scoring charts. All patients were re-interviewed six weeks after discharge, their diary and pain chart data collected and a pain assessment questionnaire completed. Data was collected and analyzed using SPSS version 20. Two- Way ANOVA was applied for the pain score analysis.

Main outcome measures: Patient reporting of pain through pain scores regarding postoperative pain.

Results: Groups A and B consisted of 50 patients each (mean age: 62.3 and 62.2 years in groups A and B respectively) who completed all steps of the study. Patients in Group A have pain scores (mean range 0.58-4.56) significantly lower than the patients in Group B (mean range 1.86-4.64) $(p \approx 0)$. Mean pain scores decreased significantly throughout the weeks for both group but this decrease was more rapid in the intervention group $(p \approx 0)$ (mean pain in group A-week 1: 4.56, week 6: 0.58). Diary analysis using Chi Squared test demonstrated that from the first week there was a significant difference in painkiller ingestion between the two groups.

Conclusions: Pharmacist intervention with advice, information and visual aids benefitted post-cardiac surgery patients by reducing their postoperative pain.

Disclosure of Interest: None Declared.

\section{HP-PC19}

New system for antibiotic prescribing controls in a university hospital: results after one year

S. Chyderiotis ${ }^{1, *}$, B. Legendre ${ }^{1}$, H. Michelon ${ }^{1}$, F. El Sayed ${ }^{2}$, I. Pierre ${ }^{3}$, M. Villart ${ }^{1}$, A. C. Crémieux ${ }^{3}$, F. Bouchand ${ }^{1}$, A.-L. Roux ${ }^{2}$, A. Dinh ${ }^{3}$

${ }^{1}$ Pharmacy, ${ }^{2}$ Microbiology Laboratory, ${ }^{3}$ Infectious Diseases Department, Hôpital Raymond Poincaré APHP, GARCHES, France

Background and Objective: The excessive and inappropriate use of antibiotics is the leading cause of the emergence of multi-drug resistant bacteria. In hospitals, several methodologies are used to control antibiotherapies. In 2011, a multi-field team was implemented in our hospital to control antibiotic prescriptions and to help physicians to correct inappropriate prescriptions as soon as possible. The objective is to achieve a more adequate antibiotic prescribing practice.

Setting and Method: The multi-field team is composed of an infectious disease specialist (IDS), a pharmacist and a bacteriologist. From February 2012 to February 2013, the prescriptions of the following drugs (called "restricted" antimicrobial drugs) were assessed: glycopeptids, daptomycin, piperacillin/tazobactam, carbapenems, ceftazidim, ciprofloxacine, levofloxacine and linezolide. Members of 
the team analyzed indications relevance, antibiotics type, dose and duration choice as well as the adjustment of the treatment regarding bacteriological results. Setting: care units, including orthopaedic surgery, medicine and rehabilitation departments.

Main outcome measures: Each day, the pharmacist collects the new antibiotic prescriptions thanks to computerized physician order entry $(\mathrm{CPOE})$ Phedra ${ }^{\circledR}$ (SIB) and enter them in an Excel $^{\circledR}$ shared document. Then, the bacteriologist fills the document with bacteriological results and his opinion on the treatment. If an inappropriate treatment is continued after $72 \mathrm{~h}$ (CPOE checked by the pharmacist) the pharmacist enters a warning in the Excel ${ }^{\circledR}$ sheet for the treatment. The IDS contacts the prescriber, and discusses it with him. Each intervention is recorded.

Results: 544 prescriptions were collected in one year of follow up. The most prescribed antimicrobials were vancomycin $(n=197)$, piperacillin/tazobactam $(n=163)$ and carbapenems $(n=60)$. From the bacteriologist's point of view, most of the initial treatments $(62 \%$ or $336 / 544$ ) were empiric therapies and therefore had to be adjusted with a more narrow spectrum antibiotic or discontinuation. This adjustment was immediately realized by the first prescriber himself without help of our team in 230 prescriptions ( $68 \%$ ). Among the 106 other prescriptions, the IDS contacted the prescriber in 75 cases. For 51 cases $(68 \%)$, the antimicrobial choice was considered justified (anterior bacterial results, penicillin hypersensitivity, clinical or epidemiological justification). 24 cases ( $32 \%$ ) were judged inappropriate and the antibiotic was changed for a more narrow spectrum antibiotic or discontinued.

Conclusions: Thanks to that organization, $94 \%$ (513/544) of antibiotic prescriptions are well adapted according to bacteriological results. This study proved that through the work of a multi-field team which can help the prescribers every day, it is possible to improve the quality of the antibiotherapy in a hospital.

Disclosure of Interest: None Declared.

\section{PDFII Pharmacotherapy}

\section{PT07}

\section{Adverse drug reactions as cause of hospital admissions} in childhood

\section{P. Langerova ${ }^{1, *}$, J. Vrtal ${ }^{1}$, K. Urbánek ${ }^{1}$ \\ ${ }^{1}$ Department of Pharmacology, Palacky University, Olomouc, Czech Republic}

Background and Objective: Knowledge of adverse drug reactions (ADRs) is crucial for safe use of medication. ADRs are common clinical problem in both pediatric and adult medicine that can lead to significant morbidity. The spectrum of ADRs in children may be rather different to that seen in adults. The aim of this study was to identify prospectively the ADRs causing hospital admission of children during a nine month period.

Setting and Method: The study was performed at the University Hospital in Olomouc, Czech Republic. Every patient younger than 19 years admitted to the pediatric clinic was assessed and all admissions for ADRs were prospectively screened for nine months from 1st March 2012 to 30th November 2012. The following information from the case files was collected: age, sex, presenting complaint, clinical history, diagnosis, medication including over-the-counter drugs taken in the preceding time. The assessment of causality was performed using Naranjo algorithm, Liverpool ADR Causality Assessment Tool and Edwards \& Aronson causality assessment method.

Main outcome measures: Quantification of the occurrence of ADRs among children admitted to hospital and identification of the risk factors and involved drugs.
Results: During the study period, 2903 admissions (1,664 boys, 1,239 girls; some of them admitted repeatedly) were identified. From the total there were 143 admissions of patients with an oncology disease (4.9\%). Sixty-five admissions $(2.2 \%)$ were caused by an ADR (28 girls and 37 boys). Twenty-eight admissions (43.1\%) caused by ADR were found in children suffering oncological diseases. Besides effects of anti-cancer chemotherapy, the most frequent ADR were vomiting and allergic reactions caused by antibiotics; the most serious were acute pancreatitis caused by anti-inflammatory drugs in IBDs and deep vein thrombosis due to combined hormonal contraception.

Conclusions: We have demonstrated that ADRs cause a small but substantial proportion of children's admissions to the hospital. Patients at highest risk are those suffering oncological diagnoses. Some of ADRs were found to be potentially avoidable. Prevention of avoidable ADRs requires careful attention to good prescribing practice.

Supported by Grant IGA UPOL LF 2013_007.

Disclosure of Interest: None Declared.

\section{PT08}

Prevalence of QT-prolongation in psychiatric hospitals in Belgium

E. Vandael ${ }^{1, *}$, J. Reyntens ${ }^{2}$, R. Willems ${ }^{3}$, J. Vandenberghe ${ }^{4}$, I. Spriet ${ }^{1}$, V. Foulon ${ }^{1}$

${ }^{1}$ Department of Pharmaceutical and Pharmacological Sciences, University of Leuven, Leuven, ${ }^{2}$ Pharmacy, Sint-Jan hospital, Eeklo, ${ }^{3}$ Department of Cardiovascular Sciences, ${ }^{4}$ Department of Neurosciences, University of Leuven, Leuven, Belgium

Background and Objective: Several cardiac and non-cardiac drugs can prolong the QT-interval, an important phase in the electrocardiogram (ECG). In rare cases, QT-prolongation can lead to ventricular arrhythmias (Torsades de Pointes) and sudden cardiac death. The list of potential QT-prolonging drugs is extensive and consists of many antipsychotic drugs and antidepressants. Therefore, psychiatric patients are a population at risk for QT-prolongation and sudden cardiac death.

The objective of this study is to obtain data on the prevalence and extent of drug-induced QT-prolongation in psychiatry.

Setting and Method: On-going observational study in 8 psychiatric hospitals in Flanders. Patients are included when a QT-prolonging drug is added to a medication profile that already contains a potential QT-prolonging drug. An ECG is taken before the administration of the new drug and a week after starting the new drug to investigate the change in duration of the QTc-interval. Risk factors for developing QT-prolongation and blood concentrations of potassium and creatinine are documented.

Main outcome measures: The duration of the QTc-interval.

Results: So far, 77 patients have been included in the study (median age: 37 years, range 19-83; $65 \%$ males). At baseline, the mean QTcinterval was $424 \mathrm{~ms}( \pm 22.3)$. 7 patients (4 males) had a QTc-interval between 450 and $470 \mathrm{~ms}, 1$ female between 470 and $480 \mathrm{~ms}$ and 1 male between 480 and $500 \mathrm{~ms}$. A week after the start of an additional QT-prolonging drug, the mean QTc-interval was $432 \mathrm{~ms}( \pm 27.6)$ with a median QTc-prolongation of $5 \mathrm{~ms}$ (range -39 to +79 ). 14 patients (7 males) had a QTc-interval between 450 and $470 \mathrm{~ms}, 2$ females between 470 and $480 \mathrm{~ms}, 2$ females between 480 and $500 \mathrm{~ms}$ and 1 female had a QTc-interval above $500 \mathrm{~ms}$. In 3 patients, a QTcprolongation of more than $60 \mathrm{~ms}$ was observed. The median number of QT-prolonging drugs per patient was 3 (range 2-6); especially antipsychotic drugs and antidepressants were involved. The most common other risk factors were smoking (68.8\%), using illegal drugs $(37.7 \%)$ and hyperkalemia $(27.3 \%)$. 
Conclusions: The first results of this study indicate a high prevalence of QT-prolongation after the administration of an extra drug with risk of QT-prolongation. 4 patients $(5.2 \%)$ had a serious risk of developing Torsades de Pointes.

Disclosure of Interest: None Declared.

\section{PT09}

Survey of evaluation in outpatient prescription of Subcutaneous Immunoglobulin

\section{G. Kreutter ${ }^{1, *}$, A.-C. Gerout ${ }^{1}$, B. Gourieux ${ }^{1}$}

${ }^{1}$ Pharmacy-sterilization department, Hôpitaux Universitaires de Strasbourg, Strasbourg, France

Background and Objective: Evaluate the effectiveness and monitoring requirements of Subcutaneous Immunoglobulin in outpatient. Setting and Method: This is a retrospective, observational study on medical records. All prescriptions of SC Immunoglobulin (SCIg) in outpatient were analyzed over a period of 15 months. Only patients with a regular treatment (at least 6 rescues on drugs over the past 15 months) and monitoring (at least 6 monthly dosage performed) were included.

Main outcome measures: The primary endpoint analysis was the serum immunoglobulin $\mathrm{G}$ trough level (nephelometry international CRM 470 standard) conducted monthly for patients. It should be between 5 and $8 \mathrm{~g} / \mathrm{l}$.

Results: Sixty patients received SCIg during the 15-month study with a $\mathrm{M} / \mathrm{F}$ ratio $=2$, and a mean age 46 years. All patients had a secondary immune deficiency. On average, patients received the treatment for 12 months.

The average trough levels of IgG was $6.7 \pm 0.5 \mathrm{~g} / \mathrm{l}$. On average, each patient had $8 \%$ of dosages $<5 \mathrm{~g} / \mathrm{l}$ and $9 \%>8 \mathrm{~g} / \mathrm{l}$. The average $\mathrm{IgG}$ trough level was optimal (between 5 and $8 \mathrm{~g} / \mathrm{l}$ ) for $88 \%$ of patients. Three percent $(3 \%)$ had an average IgG rate less than $5 \mathrm{~g} / \mathrm{l}$ and $8 \%$ higher than $8 \mathrm{~g} / \mathrm{l}$.

During the study, $39 \%$ of patients had at least a change in dosage. After the change of dosage, $92 \%$ of patients had an average level of $\mathrm{IgG}$ between 5 and $8 \mathrm{~g} / \mathrm{l}$ (against $83 \%$ previously). We also found that for patients with the same dose throughout the study, the average rate of $\mathrm{IgG}$ at the end of the study was higher than the average of the beginning of the study $(p=0.1)$.

Conclusions: The treatment was balanced because about $90 \%$ of patients had an optimal way Ig level within the therapeutic range over $80 \%$ of the time. A dose adjustment was performed to optimizing treatment. Doctors had reduced dosages of drugs and efficacy was maintained. A new study involving the clinical efficacy (infections) and tolerance should complete these results.

Disclosure of Interest: None Declared.

\section{PDFII Community pharmacy: pharmaceutical care}

\section{CP-PC02}

A framework for INR testing and anticoagulation management in community pharmacies

\section{E. M. Mifsud ${ }^{1}$, L. M. Azzopardi ${ }^{1, *}$, A. Serracino Inglott ${ }^{1}$}

${ }^{1}$ Department of Pharmacy, University of Malta, Msida, Malta

Background and Objective: The dramatic increase in the requests for International Normalised Ratio (INR) monitoring is resulting in overburdened outpatient clinics. Point-of-Care testing (POCT) devices that measure the INR, enable the monitoring to be performed outside a laboratory setting. The aims were to propose and investigate the feasibility and acceptability of a community pharmacist-led anticoagulation service and to evaluate the accuracy and clinical usefulness of the POCT device CoaguChekXS.

Setting and Method: Fifty patients taking warfarin were recruited by convenience sampling from seven community pharmacies. For each patient INR testing was performed using the CoaguChekXS POCT device on the same day that their venous plasma INR was monitored at the outpatient clinic. The level of agreement between the two INR methods was analysed using linear regression and Bland-Altman plots. Following the testing, patients completed a questionnaire designed to assess their perceptions on the current and proposed INR testing systems. Guidelines for the decentralisation of INR testing to community pharmacies were prepared and validated by a panel of eight health professionals.

Main outcome measures: Assessment of the clinical accuracy of CoaguChekXS and evaluation of patients' acceptance of the proposed testing system.

Results: Out of the 50 patients, 31 were female and 19 were male (mean age of 73 years). Linear regression analysis demonstrated a significant positive correlation between the two INR testing methods $(\mathrm{R}=0.906)$. Eighty-seven per cent of the dual measurements were within 0.5 INR units of each other. The Bland-Altman plot showed an increasing discrepancy between the two methods above the INR value of 3.5. All participants had an overall good perception of the proposed framework, with 41 patients expressing interest in using the service following implementation, and 29 patients accepting warfarin dose adjustments to be performed by community pharmacists. The multidisciplinary panel described the developed guidelines as very comprehensive, since they deal with all necessary aspects to ensure that the service is carried out safely and effectively.

Conclusions: The study provides evidence that community pharmacist-led anticoagulation monitoring is a feasible alternative to laboratory INR testing and will improve the current anticoagulation management by increasing therapeutic effectiveness and patient satisfaction.

Disclosure of Interest: None Declared.

\section{PDFII Pharmacotherapy}

\section{PT10}

Drug-Eluting Bead loaded with Irinotecan (DEBIRI): an alternative treatment of liver unresectable metastases from colorectal cancer?

A. Cransac ${ }^{1}$, M.-T. Baylatry ${ }^{1, *}$, J. Cazejust ${ }^{2}$, C. Garcia-Alba ${ }^{2}$, C. Fernandez ${ }^{1}$, A.-C. Joly ${ }^{1}$

${ }^{1}$ Pharmacy, ${ }^{2}$ Radiology, Saint-Antoine Hospital (East Parisian University Hospitals, AP-HP), Paris, France

Background and Objective: Metastatic colorectal cancer is one of the main causes of cancer related death in the world, and liver metastases are common in these patients. Surgical ablation and FOLFOX therapy (fluorouracil, oxaliplatin and leucovorin) are standard treatments for resectable liver metastases. When surgery is not possible, chemotherapy, radiofrequency ablation and intra-arterial chemotherapy are available. Fiorentini et al. have suggested that trans-arterial chemoembolization with DEBIRI (drug-eluting bead loaded with irinotecan) could be proposed for the treatment of unresectable liver metastases from colorectal cancer. This regional approach allows to increase the drug concentration while reducing late systemic toxicity. In this report, we describe a case of a woman 
with rectum adenocarcinoma treated with DEBIRI for unresectable liver recurrences.

Setting and Method: Case report from Oncology, Radiology and Pharmacy departments. Safety and efficacy preliminary results of DEBIRI are presented.

Main outcome measures: Safety evaluation: occurrence of adverse events. Efficacy evaluation: evaluation of the disease (recurrence, response).

Results: A 60-year-old woman was suffering from a metastatic adenocarcinoma of the rectum since 2005. An anterior resection of the rectum was performed, combined with FOLFOX regimen (02/ 2005-12/2005), and a surgery of liver metastasis was realized in October 2005. Liver recurrences occurred in 2006, 2008, 2010 and 2011. As surgeries have failed, several treatments were initiated (from 2006 to 2010): FOLFIRI (fluorouracil, irinotecan and leucovorin)cetuximab (05/2006-08/2006), FOLFOX-bevacizumab (10/2006-05/ 2007), LV5FU2 (fluorouracil and leucovorin)-bevacizumab (07/ 2007-01/2009), bevacizumab only (01/2009-03/2009), LV5FU2bevacizumab (08/2009-02/2010), FOLFIRI (03/2010-04/2010). These treatments were poorly tolerated by the patient. As she refused a new IV therapy, DEBIRI treatment loaded with $100 \mathrm{mg}$ of irinotecan was started in February 2012. A partial response occurred and a second embolization with $100 \mathrm{mg}$ was performed one month later. A progression was observed ten months later, she received a third injection of $100 \mathrm{mg}$ in April 2013. In May 2013, the evaluation showed a stable disease. Adverse events were observed in the days after embolizations: nausea, vomiting, pain and fever, liver enzyme elevation related to the "postembolization syndrome", and diarrhea. Conclusions: In this case, disease was stabilized after 3 embolizations with DEBIRI and a total dose of $300 \mathrm{mg}$ of irinotecan, with a tolerable toxicity. These doses are much lower in comparison with FOLFIRI for this patient $(280 \mathrm{mg}$ of irinotecan associated with $4400 \mathrm{mg}$ of fluorouracil at each cycle). This regional approach using DEBIRI seems to be a promising alternative of systemic treatment in unresectable metastases to the liver from colorectal cancer, with a likely better safety profile.

Disclosure of Interest: None Declared.

\section{PT11}

The stability of penicillin $G$ in infusion devices: a potential use as continuous infusion in outpatients (OPAT)

B. Langrée ${ }^{1,2,3, *}$, S. Patrat-Delon ${ }^{4}$, M. Dermu ${ }^{1,2}$, F. Lemaitre ${ }^{1,2}$, M. Revest ${ }^{4}$, P. Tattevin ${ }^{4}$, C. Arvieux ${ }^{4}$, M.-C. Verdier ${ }^{1,2}$, C. Michelet ${ }^{4}$, E. Bellissant ${ }^{1,2}$

${ }^{1}$ Pharmacology, Rennes University Hospital, ${ }^{2}$ Experimental and Clinical Pharmacology Department, Rennes Medical School, ${ }^{3}$ Pharmacy, Guillaume Régnier Hospital, ${ }^{4}$ Infectious Diseases Department, Rennes University Hospital, Rennes, France

Background and Objective: Because the betalactams are timedependent antibiotics, continuous infusion is optimal for their intravenous administration, especially for drugs with short half lives, as penicillin G. However, issues about drug stability may request frequent changes of infusion solutions, and may not be compatible with outpatient parenteral antimicrobial therapy (OPAT) programs. The aim of our study was to evaluate the stability of penicillin $G$ in portable medical infusion devices (ID), and to determine whether continuous infusion could be implemented within OPAT programs. Setting and Method: Three different penicillin G infusions were prepared in portable infusion devices, on three different days (10 million IU diluted in $120 \mathrm{~mL}$ of sodium chloride $0.9 \%$ ), and kept at ambient temperature, exposed to light. Two aliquots were taken after $0,1,2,3,4,6,8$, and $12 \mathrm{~h}$ and were assayed with two fully validated methods: one using liquid chromatography with ultraviolet detection (LC/UV), the other using liquid chromatography coupled tandem mass spectrometry (LC/MS-MS).

Main outcome measures: Mean penicillin $\mathrm{G}$ concentrations were measured in $\mu \mathrm{g} / \mathrm{mL}$, and were compared to $\mathrm{T}_{0}$ (initial concentration measured). Stability was defined as a decrease in concentration less than $10 \%$.

Results: The mean percentages of decrease in penicillin $\mathrm{G}$ concentrations were $0.96,2.92,4.23,4.27,7.21,2.21,8.99 \%$, respectively, for $\mathrm{T}_{1}, \mathrm{~T}_{2}, \mathrm{~T}_{3}, \mathrm{~T}_{4}, \mathrm{~T}_{6}, \mathrm{~T}_{8}$ and $\mathrm{T}_{12}$. There was a very limited interexperimental variation.

Conclusions: Penicillin $\mathrm{G}$ at usual concentrations in sodium chloride $0.9 \%$ is stable during at least $12 \mathrm{~h}$ period, at ambient temperature and exposed to light, in portable ID provided by the hospital pharmacy. This stability study allows patients to be treated at home with penicillin $G$ continuous infusion. Further studies should be implemented to explore the stability in other solvents, especially glucose. Disclosure of Interest: None Declared.

\section{PT12}

Assessment of dabigatran use in a cardiovascular setting in a tertiary hospital

H. Anglada-Martinez ${ }^{1}$, N. C. García ${ }^{1, *}$, G. Molas-Ferrer ${ }^{1}$, J. Ribas-Sala ${ }^{1}$

${ }^{1}$ Pharmacy Service, Hospital Clinic, Barcelona, Spain

Background and Objective: Dabigatran was added to our hospital's formulary (HF) in October 2012 for stroke prevention in chronic auricular fibrillation (AF). In this context, the Pharmacy and Therapeutics Committee (P\&TC) established restricted use conditions (RUC) for this drug. The aim of our study was to determine compliance degree with RUC and tolerability of dabigatran.

Setting and Method: Observational retrospective study conducted at a tertiary referral university hospital in Spain. Patients that received at least one dose of dabigatran from October 2012 to April 2013 were included.

Main outcome measures: Percentage of patients on dabigatran compliant with RUC and adequate posology based on patients specific parameters (age, weight and renal function). Rate of patients with adverse events.

Results: A total of 29 patients (19 men) were included with a mean age of $64.1 \pm 15$ years. Twenty two patients $(75 \%)$ were newly started on dabigatran and 14 of those had been previously treated with acenoucumarol. Dabigatran was prescribed to treat AF, according with P\&TC indications, in 14 patients $(48.3 \%)$ and to treat $\mathrm{AF}$ postablation anticoagulation in 15 patients $(51.7 \%)$. Appropriateness of posology was found in 15 patients $(51.7 \%)$. Within the 14 patients left, 5 had Dabigatran underdosed. Data from weight, creatinine clearence and both were abscent in $13(44.8 \%), 1(3.4 \%)$ and 7 $(24.1 \%)$ cases respectively.

One patient had a contraindication for Dabigatran because of rectorraghias and lower gastrointestinal bleeding history. During Dabigatran treatment, three patients experienced rectorrhagias resulting in one death.

Conclusions: Overall, half of the patients, who were on dabigatran, followed the P\&TC restricted use conditions. Up to $73 \%$ of the patients did not have specific parameters recorded. A high off-label use of this drug was found for AF postablation anticoagulation. These outcomes have raised concerns about dabigatran safety.

Disclosure of Interest: None Declared. 


\section{PT14}

Trastuzumab on improvement of a rare case of paraneoplastic neuropathy in a gastric cancer.

E. Campos-Davila ${ }^{1 \text {,* }}$, J. L. Puerto-Alonso ${ }^{2}$, G. Perez-Vazquez ${ }^{2}$, J. J. Ramos-Baez ${ }^{1}$

${ }^{1}$ Pharmacy, ${ }^{2}$ Internal Medicine, Hospital SAS La Línea, La Linea de La Concepcion, Spain

Background and Objective: Paraneoplastic neurologic syndromes (PNS) are disorders of the central or peripheral nervous system in the setting of cancer that is not explained by any of other mechanisms. Diagnostic criteria incorporate clinical and serological data that include the improvement after treatment. We present a rare case of sensitive-motor neuropathy in a patient with gastric cancer that improved after treatment with trastuzumab and TCF chemotherapy scheme.

Setting and Method: Description of the physical, analytical and radiological investigations and therapeutic procedures to confirm the diagnosis.

Main outcome measures: Neurologic physical exam, Cranial MRI, analytical data, electroneurography, CT scan.

Results: A 76-year-old man with history of hypertension was admitted because a 3-weeks clinical picture of dysesthesias, weakness and spontaneous fasciculation in the lower limbs. Laboratory investigations: albumin-cytological dissociation in CSF. Cranial MRI: cortical atrophy and signs of small vessels disease. Electroneurography: sensitive-motor demyelinating polineuropathy. The patient was diagnosed as a presumed Guillain-Barré syndrome, treated with immunoglobulin i.v. for eight days and discharged with weakness persistence. Patient got worse and 2 months later was readmitted because dysphagia. New laboratory tests showed: CA 19.9 2,359 $\mathrm{U} / \mathrm{mL}$; ACE $771 \mathrm{ng} / \mathrm{mL}$; CA $125139 \mathrm{U} / \mathrm{mL}$; antiganglioside and onconeural antibodies: negative. Thorax-abdominal CT scanner: gastric wall thickness, retroperitoneal adenopathies, and metastasic liver. Oral endoscopy: Irregular shaped and ulcerated lesion in lesser curvature. Biopsy: gastric adenocarcinoma Her2 positive. Surgery was not considered and treatment with trastuzumab and TCF scheme (docetaxel, cisplatin and 5-FU) was initiated. A partial, but clear, improvement was observed, although the patient died some months later.

Conclusions: Paraneoplastic sensitive-motor neuropathy is a rare entity normally diagnosed in patients with kwon and advanced neoplasms. The diagnosis is difficult because its rareness. Recently, Graus et al. have stablished diagnostic criteria that incorporate clinical and serological data. In our case the clinical improvement with trastuzumab and chemotherapy was essential to confirm the diagnosis of PNS.

Disclosure of Interest: None Declared.

\section{PT15}

Pharmacological Management of Hypertension in Diabetic Patients

J. Gypens ${ }^{1, *}$, F. Wirth ${ }^{2}$, L. M. Azzopardi ${ }^{2}$, S. Steurbaut ${ }^{1}$, S. Fava ${ }^{3}$

${ }^{1}$ Vrije Universiteit Brussel, Brussels, Belgium, ${ }^{2}$ Department of Pharmacy, Faculty of Medicine and Surgery, University of Malta,

${ }^{3}$ Diabetes and Endocrine Centre, Mater Dei Hospital, Msida, Malta

Background and Objective: The 2007 'Guidelines for the Management of Arterial Hypertension (HT)' and 'Guidelines on diabetes, pre-diabetes and cardiovascular diseases' issued by the Task Force for the Management of Arterial HT of the European Society of Hypertension and the European Society of Cardiology recommend angiotensin converting enzyme (ACE) inhibitors or angiotensin II receptor antagonists (ARBs) as first line treatment for HT in patients with diabetes mellitus. The aim of this epidemiological study was to determine whether first line pharmacological management of HT in diabetic patients in Malta is in accordance with these European guidelines.

Setting and Method: Four-week audit at Diabetes and Endocrine Centre, MDH. The guidelines were extensively scrutinised. 125 patient files were collected. Current prescribed medications, medication history, co-morbidities and laboratory investigations were reviewed and tabulated in a data collection form. Comparison to the guidelines and descriptive statistics were undertaken.

Main outcome measures: Audit of pharmacological management of HT in diabetic patients according to European guidelines.

Results: The majority of patients $(93 \%)$ were on ACE inhibitor (72\%) or ARB (21\%) therapy in accordance with the guidelines. The most frequently prescribed ACE inhibitor was perindopril $(61 \%)$, followed by enalapril (37\%). The most frequently prescribed ARB was valsartan ( $89 \%)$, followed by losartan (12\%). Of the $7 \%$ (9) of patients who were not on ACE inhibitor or ARB treatment, there was a justified reason in $6 \%$ (8) of these patients; an elevated potassium and/ or creatinine level. $32 \%$ of patients were on 1 or more diuretics (20 on bendroflumethiazide; 17 on bumetanide), $28 \%$ of patients were on a calcium channel blocker (all on amlodipine) and $19 \%$ of patients were on a beta-blocker, predominantly atenolol (21 patients).

Conclusions: Pharmacological management of HT in diabetic patients in Malta is in accordance with European guidelines for the majority of patients assessed in this audit.

Disclosure of Interest: None Declared.

\section{PDFIII Pharmacoeconomics}

\section{PEC03}

Plerixafor in multiple myeloma and lymphoma before autograft: a pharmacoeconomical perspective

A. Loison ${ }^{1, *}$, I. Borget ${ }^{2}$, C. Borel ${ }^{1}$, D. Bastit ${ }^{3}$, P. Tilleul ${ }^{4}$, H. Tilly ${ }^{5}$, F. Basuyau', R. Varin ${ }^{6}$, M. Daouphars ${ }^{1}$

${ }^{1}$ Pharmacy, Cancer Centre Henri Becquerel, Rouen, ${ }^{2}$ Service d'Etudes et Recherche en Economie de la Santé, Institut Gustave Roussy, Villejuif, ${ }^{3}$ Etablissement Français du Sang, Rouen, ${ }^{4}$ Pharmacy, GH Pitié-Salpêtrière, Paris, ${ }^{5}$ Hematology, Cancer Centre Henri Becquerel, ${ }^{6}$ Pharmacy, University hospital Charles Nicolle, Rouen, France

Background and Objective: Intensive chemotherapies are standard of care for bad prognostic multiple myeloma and lymphoma. Myelotoxicity of these protocols requires hematological rescue procedures. The critical step in this treatment is to obtain a graft containing sufficient hematopoietic stem cells for the autograft. Since 2010, Plerixafor $\left(\right.$ Mozobil $^{\circledR}$ ) has changed the management of these diseases both on the clinical and economical perspectives. This treatment is currently used to enhance mobilisation of peripheral blood stem cells (PBSC) for collection and subsequent autologous transplantation in patients whose cells mobilise poorly.

Setting and Method: We conducted a cost-effectiveness analysis comparing mobilisation strategy with plerixafor versus standard strategy with granulocyte colony stimulating factor (G-CSF) \pm chemotherapy. This retrospective monocentric study included patients treated either for multiple myeloma or lymphoma and who had a poor mobilisation of PBSC, between 2010 and 2012. We collected data on cytapheresis from 
the French Blood Service ("EFS") and economic data from our department of health information. TreeAge ${ }^{\circledR}$ software has been used to build a model, a decision tree and to make sensitivity analysis.

Results: 49 patients were included in the study: $56 \%$ men, $44 \%$ women (median age: 63 years). 22 patients were in the Plerixafor group versus 27 patients in the group without Plerixafor. No statistical difference was shown between groups regarding mobilisation risk factors. A significant difference in favour of Plerixafor is observed in the number of mobilisation, and CD34+ cells' rate. A $50 \%$ diminution of the number of cytapheresis and a faster hematologic recovery were found in the Plerixafor group. The sensitivity analysis lead to an Incremental Cost-Effectiveness Ratio (ICER) of 14,763€/autograft success. In other words, Plerixafor strategy has a $80 \%$ chance to be cost-effective if the willingness to pay is greater than $14,763 €$.

Conclusions: The overall results obtained in this first French costeffectiveness study on mobilisation strategies with or without Plerixafor show in favour of a Plerixafor use for patients who mobilised poorly their hematologic stem cells. This study can be a supportive decision tool for haematologists in the complex choice of therapeutic strategy for these patients.

Disclosure of Interest: None Declared.

\section{PDFIII Therapeutic drug monitoring and pharmacokinetics}

\section{TDMP06}

Role of genetic factors on the effect of additional loading doses and two maintenance doses used to overcome clopidogrel hyporesponsiveness

G. Latkovskis $^{1,2,3}$, I. Urtane ${ }^{4, *}$, A. Knipse ${ }^{1}$, L. Puceta ${ }^{2}$, R. Peculis ${ }^{5}$, J. Klovins ${ }^{5}$, A. Erglis ${ }^{1,2,3}$

${ }^{1}$ Latvian Center of Cardiology, Pauls Stradins Clinical University Hospital, ${ }^{2}$ Faculty of Medicine, ${ }^{3}$ Latvian Research Institute of Cardiology, University of Latvia, ${ }^{4}$ Faculty of Pharmacy, Riga Stradins University, ${ }^{5}$ Latvian Biomedical Research and Study Centre, Riga, Latvia

Background and Objective: Hyporesponsiveness to clopidogrel is associated with thrombotic events following coronary stent implantation. We aimed to investigate whether genetic polymorphisms associated with high platelet reactivity modify effect of additional loading doses (LDs) used to overcome hyporesponsiveness to clopidogrel.

Setting and Method: In a prospective single-center study we included patients after DES implantation receiving clopidogrel LD of $300 \mathrm{mg}$ or $600 \mathrm{mg}$, respectively. According to the first VASP platelet reactivity index (PRI) test patients were classified as responders or hyporesponders (PRI $<60 \%$ or $\geq 60 \%$, respectively). Hyporesponders received up to 3 additional LDs (600 $\mathrm{mg}$ ) and higher maintenance dose (MD, $150 \mathrm{mg}$ ) for one month and $75 \mathrm{mg}$ thereafter. The following polymorphisms were tested: CYP2C19*2, *3, *17, CYP2C $9 * 2, * 3$.

Results: A total of 94 patients were enrolled. Among patients $68(72.3 \%)$ were hyporesponders after the first LD. After second and third LD $24(25.5 \%)$ and $11(11.7 \%)$ patients had PRI $\geq 60 \%$, respectively. After fourth LD 2(2.1\%) patients were non-responders and were switched to ticagrelor. At the follow-up, only $8(12.3 \%)$ of hyporesponders had PRI $\geq 60 \%$ while on MD of $150 \mathrm{mg}$ compared to $33(48.5 \%)$ while on $75 \mathrm{mg} \mathrm{MD}(p<0.001)$.

Patients carrying at least one CYP2C19*2 allele had a higher PRI after the first LD than those carrying the wild-type genotype $(77.2 \pm 13.3$ vs $65.3 \pm 19.5, p=0.012)$. After the first additional LD of clopidogrel PRI decreased similarly in both groups of CYP2C19*2 genotype $(-35.3 \pm 1.9 \%$ p vs $-27.3 \pm 17.4 \%$ p, $p=0.348)$.
In carriers of CYP2C19*2 allele PRI was higher with both MD of $150 \mathrm{mg}$ and $75 \mathrm{mg}: 53.3 \pm 12.1$ vs $40.4 \pm 13.5(p=0.001)$ and $63.1 \pm 11.3$ vs $50.3 \pm 17.1(p=0.002)$, respectively.

Patients carrying at least one CYP2C19*17 allele had a lower PRI after the first LD compared with those carrying wild-type genotype $(65.1 \pm 20.7$ vs $72.5 \pm 14.9, p=0.071)$.

Conclusions: Carriers of the CYP2C19*2 less frequently reach adequate platelet inhibition and therefore require more additional LDs. The MD of $150 \mathrm{mg}$ was more effective than $75 \mathrm{mg}$ both in hyporesponders and in carriers of CYP2C19*2.

Disclosure of Interest: None Declared.

\section{TDMP07}

Should we take into account pristinamycin and tacrolimus interaction? Two case reports

M. Richard ${ }^{1}$, S. Chanoine $e^{1,2,3}$, C. Chapuis ${ }^{1}$, V. Martin', C. Pison ${ }^{4,5,6}$, B. Allenet ${ }^{1,4,7}$, P. Bedouch ${ }^{1,4,7, *}$

${ }^{1}$ Pharmacy, Grenoble University Hospital, ${ }^{2}$ INSERM, Institut National de la Santé et de la Recherche Médicale, U823, Institut Albert Bonniot, ${ }^{3}$ Joseph Fourier University, ${ }^{4}$ Joseph Fourier University, ${ }^{5}$ Chest clinic, Grenoble University Hospital, ${ }^{6}$ INSERM, Institut National de la Santé et de la Recherche Médicale, U1055, ${ }^{7}$ CNRS, Centre National de la Recherche Scientifique, TIMC-IMAG UMR 5525/Themas, Grenoble, France

Background and Objective: The consequences of drug-drug interactions may be serious, especially in transplanted patients who receive drugs with narrow therapeutic index. Immunosuppressive therapy increases the risk of infection leading to antibiotic treatments, potentially enzyme inhibitors or inducers. An interaction between pristinamycin, a synergistin group antibiotic, and tacrolimus, an anticalcineurin inhibitor has been suspected in two cases reports.

Results: Two 53 and 61 year-old men (patient A and B) have been lung transplanted for 2 and 1.5 years respectively. Maintenance immunosuppressive therapy included tacrolimus $5 \mathrm{mg} /$ day (residual blood level: $5.9 \mu \mathrm{g} / \mathrm{L}$; creatinine clearance (MDRD): $79 \mathrm{~mL} / \mathrm{min}$ ), mycophenolate mofetil $750 \mathrm{mg} /$ day, and prednisone $30 \mathrm{mg} /$ day for patient $\mathrm{A}$; and tacrolimus $2 \mathrm{mg} /$ day (residual blood level: $3.6 \mu \mathrm{g} / \mathrm{L}$; creatinine clearance (MDRD): $91 \mathrm{~mL} / \mathrm{min}$ ), everolimus $4 \mathrm{mg} /$ day, mycophenolate mofetil $500 \mathrm{mg} / \mathrm{day}$, and prednisone $8 \mathrm{mg} /$ day for patient B. A methicillin-sensitive Staphylococcus Aureus strain has been detected in bronchoalveolar lavage $\left(10^{5}-10^{6} \mathrm{CFU} / \mathrm{mL}\right)$ in both patients without clinical sign. Pristinamycin ( $3 \mathrm{~g} /$ day) has been introduced during 7 (patient B) to 10 days (patient A). An overdose of tacrolimus has been detected: $20.3 \mu \mathrm{g} / \mathrm{L} 5$ days after discontinuation therapy for patient A (therapeutic level: 5-10) and $11.5 \mu \mathrm{g} / \mathrm{L}$ after 4 days of antibiotic (therapeutic level: $3-4$ ), corresponding to a 2.5 and 2.2-fold increase, respectively. Tacrolimus dose has been adapted ( $4 \mathrm{mg} /$ day for patient $\mathrm{A} ; 1 \mathrm{mg} /$ day after skip a dose for patient $\mathrm{B}$ ) and close monitoring has been planned. Concerning patient A, tacrolimus residual blood level has been decreased to a therapeutic level 3 days after dose adjustment, no significant impairment of renal function has been observed. The monitoring of patient $B$ is still in progress after pristinamycin discontinuation (6 days of therapy).

Conclusions: These case reports suggest an interaction between tacrolimus and pristinamycin. Drug-drug interactions have already been reported between pristinamycin and another anti-calcineurin inhibitor, cyclosporin. The interaction mechanism is not defined, although synergistins are related to macrolides which are CYP 3A4inhibitors. Interindividual variability in this interaction should be considered, especially in lung transplanted patients. A close monitoring should be performed when a synergistin is introduced in these patients. Disclosure of Interest: None Declared. 


\section{TDMP08}

Baseline (anti-)infliximab serum trough levels do not predict successful down-titration or cessation of infliximab in Rheumatoid Arthritis patients with long term low disease activity

A. Van der Maas', B. Van Den Bemt ${ }^{2, *}$, F. Van der Hoogen ${ }^{1}$, P. Van Riel ${ }^{3}$, A. Den Broeder ${ }^{1}$

${ }^{1}$ Department of Rheumatology, ${ }^{2}$ Department of Pharmacy, Sint Maartenskliniek, ${ }^{3}$ Department of Rheumatology, Radboud University Medical Center, Nijmegen, Netherlands

Background and Objective: Several studies have shown that downtitration of infliximab is feasible in a part of the patients with stable RA treatment and stable disease activity. However since down-titration can also cause flares, predictors for successful down-titration are warranted. Since infliximab serum trough levels are associated with clinical effect, they might aid in predicting successful down-titration. Therefore, we conducted a prospective cohort study to investigate if baseline infliximab serum trough levels and/or anti-infliximab-antibodies can predict successful infliximab down-titration or cessation in RA patients with low disease activity.

Setting and Method: RA patients treated with infliximab who had stable low disease activity defined as a DAS28 of $<3.2$ and stable RA treatment for more than 6 months were included in a prospective cohort study. Inclusion was from the 1st of January 2010 until 1st of April and patients were followed for a year. In all patients, infliximab was down-titrated with $25 \%$ of the original dose $(3 \mathrm{mg} / \mathrm{kg})$ every 8-12 weeks without interval change until cessation or flare. At baseline infliximab serum trough level and anti-infliximab antibodies were measured. Infliximab levels below $1.0 \mathrm{mg} / \mathrm{l}$, between 1.0 and 5.0 and above 5.0 were categorized as low, normal and high levels. Main outcome measures: The main outcome measure was the proportion of patients with low, normal and high infliximab serum trough levels in which infliximab stopped, partly down titrated or not-down titrated.

Results: In 51 RA patients, with a mean baseline DAS28 of 2.5 (sd 0.7 ) and median disease duration of 12 years [9-18], infliximab was down-titrated. After 1 year follow-up 8 patients could stop infliximab, 22 had partial down titrated and the rest returned to the baseline dose. Low serum trough level at baseline were detected in 23 patients $(45 \%$, $95 \% \mathrm{CI}=31-59 \%), 17$ had levels between $1.0 \mathrm{mg} / \mathrm{l}$ and $5 \mathrm{mg} / \mathrm{l}$ $(33 \%, 95 \% \mathrm{CI}=20-46)$ and 11 had high infliximab serum trough levels $(22 \%, 95 \% \mathrm{CI}=10-33)$. There was no statistical significant difference in baseline serum trough levels between patients in the groups 'infliximab cessation', 'partly down-titrated' or 'no down-titration' ( $p=0.18$ ), with median serum trough level at baseline of 1.0 (IQR $0.3-1.1$ ), 1.7 (IQR 0.54-5.1) and 0.55 (IQR 0.03-2.35) respectively. Conclusions: Baseline infliximab serum trough levels are not useful as an aid in the clinical decision making for down-titration for prediction of successful down-titration or cessation of infliximab.

Disclosure of Interest: None Declared.

\section{PDFIII Drug information}

\section{DI01}

Assessment of completeness and applicability of summaries of product characteristics regarding information on fertility and drug use in pregnancy and breastfeeding

\section{B. Arguello ${ }^{1, *}$, T. M. Salgado ${ }^{1}$, F. Fernandez-Llimos ${ }^{2}$}

${ }^{1}$ Research Institute for Medicines and Pharmaceutical Sciences (iMed.UL), ${ }^{2}$ Department of Social Pharmacy, Faculty of Pharmacy, University of Lisbon, Lisbon, Portugal., Lisbon, Portugal
Background and Objective: Whenever drug therapy is considered during pregnancy, both potential teratogenic risks of treatment and the potential risks of no treatment for the mother have to be carefully weighed. The European Union highlights the importance of marketing authorisation holders providing recommendations for use of drugs during pregnancy and lactation and for women of child bearing potential. The aim was to assess completeness and applicability of Summaries of Product Characteristics (SmPCs) regarding information on fertility and drug use in pregnancy and breastfeeding.

Setting and Method: SmPCs of all the medicines approved by the European Medicines Agency (EMA) were gathered from the Agency's website. SmPCs of medicines not intended to be used in pregnant women were excluded. Information provided in Sect. 4.6 (Fertility, pregnancy and lactation) of SmPCs was analysed independently by two researchers using an ad-hoc created form. Applicability of SmPCs was measured using the conclusiveness of the recommendations on the use of the medicine in pregnancy and lactation. Statements such as 'contraindicated' or 'no restrictions to the use' were considered conclusive. Recommendations such as 'use with caution' or 'use if clinically needed' were considered not conclusive. Main outcome measures: Completeness and applicability of information on the use of drugs in pregnancy and lactation provided in SmPCs. Results: Of the $582 \mathrm{SmPCs}$ in the EMA website, 48 were excluded. Of 534 analysed SmPCs, $3.3 \%$ SmPCs provided clear information about the effects of the drug in fertility, $10.7 \%$ contained information about the drug crossing the placental barrier, $17.1 \%$ SmPCs provided information about milk excretion of the drug, $16.7 \%$ SmPCs informed about clinical experience during pregnancy, and $12.7 \%$ SmPCs included clear information about management of exposure. The use of the drug was restricted in pregnant women in $94.6 \%$ of the SmPCs and in nursing women in $92.1 \%$ SmPCs. Of the SmPCs providing a recommendation for the use of the drug during pregnancy, $43 \%$ were conclusive recommendations. For breastfeeding $83.5 \%$ of the recommendations were conclusive.

Conclusions: Information on fertility, pregnancy and lactation in SmPCs is sparse. Despite the lack of information, SmPCs are very restrictive in their recommendations of use. A high percentage of SmPCs contain non-conclusive recommendations for the use of a drug during pregnancy.

Disclosure of Interest: None Declared.

\section{PDFIII Therapeutic drug monitoring and pharmacokinetics}

\section{TDMP09}

Evaluation of therapeutic drug monitoring service for theophylline

N. Rugelj ${ }^{1}$, K. Cvan Trobec ${ }^{2, *}$, M. Pislar ${ }^{1}$, P. Mesko Brguljan' ${ }^{2}$, M. Kosnik ${ }^{2}$, A. Mrhar ${ }^{1}$

${ }^{1}$ Faculty of Pharmacy, University of Ljubljana, Ljubljana, ${ }^{2}$ University Clinic Golnik, Golnik, Slovenia

Background and Objective: Theophylline is a methylxantine with spasmolytic and anti-inflammatory effects, used in the treatment of chronic obstructive pulmonary disease. Therapeutic monitoring of theophylline levels is required due to its narrow therapeutic range and marked pharmacokinetic variability. We aimed to evaluate therapeutic drug monitoring service for theophylline in our clinical setting, which currently integrates no pharmacokinetic evaluation of measured theophylline concentrations.

Setting and Method: We retrospectively evaluated 127 randomly selected theophylline serum level determinations performed in 2010 
in a tertiary clinical setting in Slovenia. Demographic data, information on theophylline dosing and blood sampling was collected from patients' data files. On the basis of collected data, population pharmacokinetic model for theophylline was built and further used for the evaluation of dosage adjustments. Paired sample t-test was used to compare actual and optimal daily doses.

Main outcome measures: Appropriateness of indications for theophylline concentration measurement, timing of blood sampling and dosage adjustments made after theophylline levels had been reported. Results: Out of 127 cases, 107 (84.3\%) had clinically justified indication for theophylline level measurement. Near half of measurements $(44.9 \%)$ were performed before the steady state of theophylline concentrations was established. $65 \%$ of measured concentrations were subtherapeutic and the average measured concentration was below therapeutic range $(53.1 \mu \mathrm{mol} / \mathrm{L})$. Despite subtherapeutic concentrations the dose of theophylline was mainly not increased. Pharmacokinetic model enabled the calculation of average optimal daily dose which was significantly higher than the average actual daily dose used (876 mg vs. $572 \mathrm{mg}, p<0.001$ ).

Conclusions: Theophylline TDM service should be optimized in our clinical setting. We suggest integration of pharmacokinetic interpretation of theophylline levels into clinical practice.

Disclosure of Interest: None Declared.

\section{TDMP10}

\section{Assessment of gentamicin dosing regimens in newborns treated} with hypothermia

P. Riera ${ }^{1, *}$, N. Solé ${ }^{1}$, E. Fernández de Gamarra ${ }^{1}$, E. Moliner ${ }^{1}$, M. J. García ${ }^{1}$, M. A. Mangues ${ }^{1}$

${ }^{1}$ Hospital de la Santa Creu i Sant Pau, Barcelona, Spain

Background and Objective: Therapeutic hypothermia has demonstrated positive outcomes in the management of newborns with hypoxic ischemic encephalopathy (HIE). Moreover, gentamicin is commonly used for the empiric treatment of presumed infection in newborns, but the effect of the cooling treatment on its pharmacokinetics is not well known. The aim of our study is to identify the optimal dosing regimen of gentamicin in newborns with HIE treated with hypothermia.

Setting and Method: This is a retrospective study in newborns with HIE treated with hypothermia at a tertiary neonatal intensive care unit. We included full-term neonates born between May 2012 and May 2013 who had received at least one dose of gentamicin during the cooling treatment. Following our protocol therapeutic drug monitoring was conducted during the first $48 \mathrm{~h}$ of treatment, pharmacokinetic studies were performed applying a Bayesian method and individual dosing adjustments were done when necessary. Dosing regimens were considered optimal when they assured the target concentrations (peak $>7 \mathrm{mg} / \mathrm{L}$ and trough $<1.5 \mathrm{mg} / \mathrm{L}$ ).

Main outcome measures: Trough gentamicin serum concentrations. Peak gentamicin serum concentrations.

Results: Six full-term neonates were included in the study. Median birth weight was $3.50 \pm 0.55 \mathrm{~kg}$ and baseline creatinine clearances were normal in the six cases. All of them started the gentamicin treatment with a dose of $4 \mathrm{mg} / \mathrm{kg} / 24 \mathrm{~h}$ administered intravenously over $30 \mathrm{~min}$, following the dosing recommendations for full-term newborns. We performed a pharmacokinetic study and simulated the concentrations that would be achieved with two different regimens ( $4 \mathrm{mg} / \mathrm{kg} / 24 \mathrm{~h}$ and $4 \mathrm{mg} / \mathrm{kg} / 36 \mathrm{~h}$ ). We observed that five of the patients $(83.3 \%)$ required an extended interval of $4 \mathrm{mg} / \mathrm{kg} / 36 \mathrm{~h}$ to achieve the target levels. The predicted peak concentrations were optimal with both regimens. The median predicted trough concentrations were $2.13 \pm 0.53 \mathrm{mg} / \mathrm{L}$ and $0.87 \pm 0.31$ with the 24 and $36 \mathrm{~h}$ intervals respectively.
Conclusions: According to our results, full-term newborns with HIE might need and extended dosing regimen of gentamicin $(4 \mathrm{mg} / \mathrm{kg}$ / $36 \mathrm{~h}$ ) to achieve target peak and trough concentrations.

Disclosure of Interest: None Declared.

\section{PDFIII Drug information}

\section{DI02}

Is information for dose adjustment in renal impairment contained in Summaries of Product Characteristics suitable for integration in clinical decision support systems?

T. M. Salgado ${ }^{1}$, B. Arguello ${ }^{1}$, F. Martinez-Martinez ${ }^{2}$, S. I. Benrimoj ${ }^{3}$, F. Fernandez-Llimos, ${ }^{4, *}$

${ }^{1}$ Research Institute for Medicines and Pharmaceutical Sciences (iMed.UL), Faculty of Pharmacy, University of Lisbon, Lisbon, Portugal, ${ }^{2}$ Cátedra de Atención Farmacéutica, University of Granada, Granada, Spain, ${ }^{3}$ Graduate School of Health, University of Technology Sydney, Sydney, Australia, ${ }^{4}$ Department of Social Pharmacy, Faculty of Pharmacy, University of Lisbon, Lisbon, Portugal

Background and Objective: Electronic versions of Summaries of Product Characteristics (SmPCs) have been proposed to integrate clinical decision support systems. The aim was to assess the applicability into clinical practice of the instructions for dose adjustment in renal impairment provided in current SmPCs.

Setting and Method: SmPCs of all the medicines approved by the EMA were retrieved from the EMA's website and instructions for use in renal impairment were analysed by two independent authors. SmPCs were classified as containing 'explicit 'or 'poor' information whether they presented or not instructions for the use in renal impairment. Information was considered 'applicable' if SmPCs provided clear instructions for dose adjustment such as 'adjust dose', 'contraindicated in renal impairment' or 'monitor patient'. Statements like 'use with caution', 'not recommended', 'should not' or 'avoid' were considered 'not-applicable' information.

Main outcome measures: Applicability of instructions for dose adjustment in renal impairment.

Results: Of the 356 SmPCs analysed, $13.8 \%$ were classified as providing poor information and $37.6 \%$ presented explicit but not-applicable information. Only $48.6 \% \mathrm{SmPCs}$ provided explicit and applicable information for medicine use in renal impairment. No difference was found in the average time since last update among SmPCs classified as containing explicit or poor information, as well as those classified as containing applicable or not-applicable information. Around $80 \%$ SmPCs did not provide information on the use of the medicine in patients undergoing hemodialysis. Of the 732 recommendations presented in the SmPCs analysed, only $256(35.0 \%)$ mentioned creatinine clearance upper limits and $174(23.8 \%)$ creatinine clearance lower limits to support the definition of a given stage of renal impairment.

Conclusions: Current SmPCs versions present several information deficits regarding dose adjustment in renal impairment, which might limit their future integration into clinical decision support systems. Disclosure of Interest: None Declared.

\section{PDFIII Pharmacoeconomics}

\section{PEC04}

Use of Telaprevir/Boceprevir on restricted economical conditions. E. Campos-Davila ${ }^{1, *}$, J. J. Ramos-Baez ${ }^{1}$, F. Tellez-Perez ${ }^{2}$ 
${ }^{1}$ Pharmacy, ${ }^{2}$ Infectious Disease Unit, Campo de Gibraltar Health Care Area, La Linea de La Concepcion, Spain

Background and Objective: A Local Commission (LC), formed by a digestologist, an Infectious Disease specialist, and a Hospital Pharmacist, was created in our Health Care Area in order to adequate the prescriptions for the use of direct-acting antiviral agents (DAAs) for the treatment of HCV genotype 1 infection in mono and co-infected patients. By agreement between the Medical Director, Pharmacy, Digestology and Infectious Disease Services, patients considered eligible for treatment with DAAs were those with F3-F4 fibrosis stage and those relapsers whose liver stiffness were $>8.5 \mathrm{kpa}$. In this study we evaluate the economical impact of the decisions taken by the LC during one year.

Setting and Method: Observational, retrospective study. Medical records and analytical data of all patients with a request of treatment with DAAs were reviewed as well as the decision taken by the LC. Data analysis was developed using SPSS statistical package.

Main outcome measures: Viral genotype, liver stiffness by ultrasound based transient elastography (fibroScan ${ }^{\circledR}$ ), IL-28 polymorphism, patient classification based on previous treatment with peginterferon-ribavirin (naïve, relapser, partial-responder, null-responder or with unknown previous response), LC decision (accepted/denied).

Results: LC evaluated 49 treatment requests. Depending on fibrosis stage, patients were F0-F2 (24.48 \%), F3-F4 (57.14\%), and $18.36 \%$ had no recent fibrosis test (neither fibroScan ${ }^{\circledR}$ nor biopsy). Depending on previous treatment, they were naïve $(44.89 \%)$, relapsers $(40.81 \%)$, partial-responders $(10.20 \%)$ and null-responders $(4.08 \%)$. Only $61.22 \%$ of patients were considered eligible for triple therapy with DAAs, being denied to 19 patients. $93.3 \%$ of accepted prescriptions corresponded to F3-F4 patients and the remaining $6.6 \%$ were two exceptions included on current HCV therapy recommendations of the Spanish Health System (one F2-naïve woman with childbearing intentions and one F2-naïve man with special working conditions).

Conclusions: The creation of a LC and its decisions in order to prioritize triple therapy with DAAs to those patients with more advanced liver disease and deferring the ones in mild stages (hoping for new, better and safer drugs) has supposed an important cost saving of $760.000 €(40.000 €$ cost-per-patient triple therapy estimation) in our Health Care Area, what is an important issue for its sustainability on the current economical conditions.

Disclosure of Interest: None Declared.

\section{PDFIII Research development}

\section{RD01}

Physico-chemical and microbiological stability studies of a melatonin oral suspension for pediatric use.

\section{J. Zerbit ${ }^{1, *}$, J. Bordenave ${ }^{2}$, G. Benoit ${ }^{2}$}

${ }^{1}$ Control Laboratory, ${ }^{2}$ Armand Trousseau Hospital, Paris, France

Background and Objective: Melatonin is a major drug for the treatment of attention deficit, hyperactivity or sleep disorders in pediatrics, a population of patients for which oral suspension is the most appropriate dosage form. The aim of this study was to evaluate the physico-chemical and microbiological stabilities of melatonin suspension in a commercially available vehicle (Inorpha ${ }^{\circledR}$ ).

Setting and Method: For physico-chemical analysis, a $2 \mathrm{mg} / \mathrm{mL}$ suspension of melatonin was divided into one hundred and twenty $5 \mathrm{~mL}$-tinted glass vials. Half was stored at $4{ }^{\circ} \mathrm{C}$ and the other half at $25{ }^{\circ} \mathrm{C}$. For each temperature, concentration of three vials was assessed weekly, in duplicate, during 18 weeks by a validated method using High Performance Liquid Chromatography with ultraviolet detection. A final measurement of melatonin concentration after 6 months at $25^{\circ} \mathrm{C}$ and a forced degradation study were also conducted.

The microbiological study was performed over a period of 4 months (7 analyses). Two packagings were tested: $10 \mathrm{~mL}$-vials in order to evaluate the microbiological stability before opening and $200 \mathrm{~mL}$-bottle opened at regular intervals in order to evaluate the stability under conditions of use. Microbial contamination was assessed using membrane filtration followed by culture on solid media allowing bacteria and fungi growth.

Main outcome measures: Physico-chemical stability was defined by a concentration above $95 \%$ of the initial concentration. Suspensions were considered microbiologically stable if Total Aerobic Microbial Count (TAMC) was less than $200 \mathrm{CFU} / \mathrm{mL}$ without any E. coli and if total combined yeasts and molds count was less than $20 \mathrm{CFU} / \mathrm{mL}$.

Results: $97.7 \%$ of melatonin initial concentration was maintained after the 18-week storage period. There was no significant difference between the 2 temperatures tested. After 6 months, melatonin concentration was $95.5 \%$ of initial concentration. The forced degradation assay showed melatonin sensitivity to alkaline $\mathrm{pH}$ and oxidation. The highest TAMC obtained during the 4-month microbiological study was $0.3 \mathrm{CFU} / \mathrm{mL}$ (no E.coli) and no yeast or mold growth was noted.

Conclusions: Results of this stability study led to set the shelf life of melatonin suspension to 3 months. The microbiological quality was not altered by repeated openings of the bottle over the study period allowing production of multidose vials.

Disclosure of Interest: None Declared.

\section{PDFIII Pharmacoepidemiology}

\section{PE02}

Type 2 diabetes mellitus and the risk of incident gout

S. G. Bruderer ${ }^{1,2, *}$, S. S. Jick ${ }^{3}$, M. Bodmer ${ }^{1,4}$, C. R. Meier ${ }^{1,2,3}$

${ }^{1}$ Basel Pharmacoepidemiology Unit, Division of Clinical Pharmacy and Epidemiology, Department of Pharmaceutical Sciences, University of Basel, ${ }^{2}$ Hospital Pharmacy, University Hospital Basel, Basel, Switzerland, ${ }^{3}$ Boston Collaborative Drug Surveillance Program, Boston University School of Public Health, Lexington, United States, ${ }^{4}$ Division of General Internal Medicine, Bern University Hospital, Inselspital, Bern, Switzerland

Background and Objective: Diabetes mellitus has been associated with a decreased risk of incident gout. However, available data about the role of diabetes severity, diabetes duration, and different antidiabetic drug treatments on the risk of incident gout in patients with type 2 diabetes mellitus (T2DM) remain limited in the medical literature.

We aimed at exploring the risk of incident gout in patients with T2DM in association with diabetes severity, diabetes duration, and different treatment modalities such as diet only, oral anti-diabetic drugs, or use of insulin.

Setting and Method: We conducted a case-control study in patients with T2DM derived from the UK-based Clinical Practice Research Datalink (CPRD). We identified case patients aged between 18 and 80 years with an incident diagnosis of gout between 1995 and 2009 and matched to each case patient one gout-free control patient on age, sex, body mass index (BMI), general practice, calendar time, and years of history in the database. We used conditional logistic regression to calculate adjusted odds ratios (adj. ORs) with $95 \%$ confidence intervals (CIs). We adjusted for potential confounders such as duration of T2DM, A1C level, and co-medication. 
Results: The study encompassed 7,538 cases with T2DM with a firsttime diagnosis of gout and the same number of gout free controls. Compared to an A1C level of $<7 \%$, increasing $\mathrm{A} 1 \mathrm{C}$ values $(7.0-7.9 \%, 8.0-8.9 \%,>9.0 \%)$ were associated with decreased relative risk estimates of incident gout [adj. OR $0.85(95 \% \mathrm{CI}$ $0.78-0.93), \quad 0.67 \quad(95 \%$ CI $0.59-0.76)$, and $0.46 \quad(95 \%$ CI $0.40-0.52)$ ], respectively. Compared to a diabetes duration $<1$ year, prolonged diabetes duration ( $1-3,3-7$, and $>7$ years) was associated with decreased adj. ORs of 0.87 (95\% CI 0.76-1.00), 0.71 (95\% CI $0.63-0.81$ ), and 0.59 (95\% CI 0.54-0.66), respectively. Use of metformin (adj. OR 0.78, $95 \%$ CI 0.72-0.85) and sulfonylureas (adj. OR $0.86,95 \%$ CI 0.79-0.93) was associated with a decreased relative risk estimate, while insulin (adj. OR 0.98, $95 \%$ CI 0.87-1.10) was not associated with an altered relative risk estimate.

Conclusions: Diabetes severity, prolonged diabetes duration, and use of metformin or sulfonylureas, but not insulin are associated with a substantially decreased risk of incident gout among patients with T2DM.

Disclosure of Interest: None Declared.

\section{PDFIII Pharmacotherapy}

\section{PT16}

Predictors associated with inappropriate antibiotic prescribing in primary care

\section{R. Fernández-Urrusuno ${ }^{1, *}$, M. Flores-Dorado ${ }^{1}$,} A. Vilches-Arenas ${ }^{2}$, M. C. Montero-Balosa ${ }^{1}$

${ }^{1}$ Farmacia, Distrito Sanitario Aljarafe, Mairena del Aljarafe, ${ }^{2}$ Epidemiology, Distrito Sanitario Sevilla, Sevilla, Spain

Background and Objective: Improving the appropriate use of antibiotics is an increasing priority for health policy and health services. Most studies on this field focused on the assessment of appropriateness of antibiotic prescribing without investigating predictors associated with quality of antibiotic prescribing. Our objective was to assess the prevalence and hypothetical predictors of inappropriateness antibiotic prescribing in Primary Care, related to patient's characteristics and antibiotic treatments.

Setting and Method: Cross-sectional study carried out in Aljarafe Primary Health Care Area (Spain), a rural/suburban area with 368,728 inhabitants assisted by 176 general practitioners and 45 paediatricians. Patients with antibiotic prescriptions and diagnosis of infection during 2009 were selected by simple random sampling (confidence level: $95 \%$; accuracy:5\%). Logic regression analysis was carried out to identify hypothetic predictors of inappropriate antibiotic prescribing associated with patient's and antibiotic treatment's characteristics. Main outcome measures: Appropriateness of antibiotic prescriptions to recommendations.

Results: Random sample included 703 patients. The most common diagnoses were respiratory $(67.6 \%$; $95 \%$ CI 64.0-71.1), urinary (14.5\%; $95 \%$ CI 11.8-17.2) and skin (11.5\%; $95 \%$ CI 9.1-13.9) infections. Beta-lactams were the antibiotic group most frequently prescribed $(77.2 \%, 95 \%$ CI 74.1-80.4), followed by fluorquinolones (8.5\%; $95 \%$ CI 6.4-10.7) and macrolides (7.0\%; $95 \%$ CI 5.0-8.9). Antibiotic prescribing was appropriate in $35.8 \%$ (95\% CI 32.2-39.5) of cases. The main reasons for inappropriateness were: wrong treatment duration, selection of inadequate antibiotic and unnecessary antibiotic use. Appropriateness did not vary by patient age, gender and comorbidity. Factors associated with inappropriateness were the type of infection, antibiotic prescribed and the number of antibiotic treatments: urinary $[\mathrm{OR}=4.3 ; 95 \% \mathrm{CI} 2.8-6.7 ; p<0.001]$ and dental infections [OR $=3.5 ; 95 \%$ CI $1.5-8.4 ; p<0.005]$ were more likely to treated appropriately. Respiratory $[\mathrm{OR}=0.4 ; 95 \% \mathrm{CI}$ $0.3-0.6 ; p<0.001]$ and skin infections [OR $=0.8 ; 95 \%$ CI $0.5-1.4$; $p=0.455$, no significative] were associated with an increased chance of inappropriateness. Regarding prescribed antibiotics, fosfomycin $[\mathrm{OR}=4.8 ; 95 \%$ CI 1.6-14.8; $p=0.006]$, azithromycin $[\mathrm{OR}=2.1$; $95 \%$ CI $1.1-4.2 ; p=0.033$ ] and tetracyclines [OR $=2.3 ; 95 \% \mathrm{CI}$ $1.3-4.2 ; p=0.004]$ were the most appropriated prescribed. Amoxicillin $[\mathrm{OR}=0.4 ; 95 \%$ CI $0.3-0.6 ; p<0.001]$ and amoxicillinclavulanate $[\mathrm{OR}=1.4 ; 95 \%$ CI $1.0-1.9 ; p<0.046]$ were associated with the lower percentage of appropriateness. Patients receiving more than one antibiotic treatment were more likely to be treated appropriately $[\mathrm{OR}=1.6 ; 95 \%$ CI $1.2-2.2 ; p=0.02]$.

Conclusions: There is a high degree of inappropriate antibiotic prescribing in Primary Care. The analysis of factors associated with inappropriateness should serve for guide the implementation of improvement actions.

Disclosure of Interest: None Declared.

\section{Community pharmacy: pharmaceutical care}

\section{CP-PC05}

The community pharmacists could increase the compliance and clinical outcomes in hypertonic polypragmatic elderly patients

A. Olearova ${ }^{1, *}$

${ }^{1}$ Department of Organization and Management in Pharmacy, Faculty of Pharmacy, Comenius University in Bratislava, Bratislava, Slovakia

Background and Objective: The poor compliance and adherence of elderly polypragmatic patients were described in various studies. The regular interventions of the community pharmacist such are communication of drugs, drug schedules preparing, identifying and solving of drug-related problems and blood pressure measuring showed the increasing compliance/adherence to the pharmacotherapy, improvement of clinical outcomes-level of blood pressure and satisfaction of patients.

Setting and Method: Four community pharmacies in Slovakia; 98 patients (70 male, 28 female), 66-85 years old, hypertonic, three and more drugs prescribed; 24 -months prospective study. Patients came every month into the community pharmacy to pick up their drugs, to measure the blood pressure, and to consult with the pharmacist why and how to take prescribed drugs. The pharmacist check out the adherence to the prescribed pharmacotherapy by the help of Morisky score and evidenced all data. Correlation between the blood pressure levels and levels of adherence was calculated by F-test.

Main outcome measures: The aim of the study was to find out the correlation between blood pressure levels before and after the regular pharmaceutical care; and level of adherence in elderly hypertonic and polypragmatic patients. Pharmacotherapy costs and number of used drugs was observed, too.

Results: Ninety-eight polypragmatic elderly patients showed very poor adherence at the beginning of the study (overall 3.5-nonadherence). The adherence increased in 6th month up to 2.64-lower partial adherence, in 12th month up to 1.21 - upper partial adherence and in 24th month up to 1.14-almost full adherence. The blood pressure showed decreasing levels: systolic blood pressure at the beginning was $148.2 \mathrm{mmHg}$ to $146.1 \mathrm{mmHg}$ in 6th month, $138.9 \mathrm{mmHg}$ in 12th month and $130.9 \mathrm{mmHg}$ in 24th month in general. Diastolic blood pressure at the beginning was $96.2 \mathrm{mmHg}$, $94.0 \mathrm{mmHg}$ in 6th month, $91.8 \mathrm{mmHg}$ in 12th month and $88.7 \mathrm{mmHg}$ in 24th month in general. F-test calculated statistically significant correlation between the blood pressure and adherence 
levels during the study $(\alpha<0.001)$. Number of used drugs went down in $9 \%$ (from 4.5 to 4.1 of drugs per patient in general). The pharmacotherapy costs were balanced.

Conclusions: The role of the pharmacist in hypertension therapy outcomes in elderly patients is very important. Results showed the regular and qualitative pharmaceutical care in community pharmacy could lead to the improvement of the blood pressure levels and adherence to the pharmacotherapy.

Disclosure of Interest: None Declared.

\section{CP-PC07}

The Beliefs about Medicines Questionnaire as predictor of medication review findings

A. Madanelo ${ }^{1}$, M. M. Castel-Branco ${ }^{1,2}$, M. M. Caramona ${ }^{1,2}$, I. V. Figueiredo ${ }^{1,2}$, F. Fernandez-Llimos ${ }^{3,4, *}$

${ }^{1}$ Pharmacology and Pharmaceutical Care, Faculty of Pharmacy, University of Coimbra, ${ }^{2}$ Center for Pharmaceutical Studies, University of Coimbra, Coimbra, ${ }^{3}$ Research Institute for Medicines and Pharmaceutical Sciences (iMed.UL), University of Lisbon,

${ }^{4}$ Department of Social Pharmacy, Faculty of Pharmacy, University of Lisbon, Lisbon, Portugal

Background and Objective: A medication review service can be a suitable situation to identify non-adherent patients, as well as to find out the type of non-adherence, which allows tailoring the recommendations. The Beliefs about Medicines Questionnaire (BMQ) is a useful instrument to assess the beliefs that will influence patients' attitudes, and subsequently determine their behaviour. The aim of this study was to assess the BMQ as a proxy for the need of patient counselling in a medication review service.

Setting and Method: Patients visiting a community pharmacy in Portugal and eligible for medication review following the UK-NHS criteria were invited to participate. Three questions of the Mini Mental Status Examination (MMSE) were used to exclude patients with cognitive impairment. Medication review was performed between Jan-Dec 2012 following the Pharmaceutical Society of Australia Guidelines. The BMQ and the MAT (a validated Portuguese self-reported adherence questionnaire) were also administered to the patients. Correlations between the BMQ and the MAT and between the BMQ and the number of recommendations provided to the patients were explored.

Main outcome measures: BMQ-necessities (BMQn), BMQ-concerns (BMQc) and MAT scores; number of recommendations resulting from the medication review.

Results: A total of 46 patients received the medication review service during the study period and three were excluded due to cognitive impairment. The mean age of participants was 78.4 years-old $(\mathrm{SD}=6.1)$, being $60.5 \%$ females, and taking a mean of 10.2 medicines $(\mathrm{SD}=3.7)$. A total of 603 findings (situations where counselling or referral was needed) resulted from the medication review process, being $186(30.8 \%)$ recommendations provided only to the patient during the interview.

The mean BMQ scores for both subscales were: $20.1(\mathrm{SD}=2.6)$ for necessity $(\mathrm{BMQn})$ and $14.3(\mathrm{SD}=4.7)$ for concerns $(\mathrm{BMQc})$. The difference BMQn-BMQc was $5.8(\mathrm{SD}=5.3)$. The mean MAT score was $38.1(\mathrm{SD}=3.0)$. As expected, MAT and BMQn-BMQc significantly correlated (Pearson $p<0.001 ; \mathrm{R}=0.556$ ). A significant correlation appeared between the BMQ (BMQn-BMQc) and recommendations to patients ( $p=0.004, \mathrm{R}=-0.425)$.

Conclusions: The BMQ is a valid tool to assess patient beliefs that may lead to intentional non-adherent behaviours in the elderly receiving medication review.

Disclosure of Interest: None Declared.

\section{CP-PC08}

What do patients think about multidrug punch cards? A mixed methods approach

F. Boeni ${ }^{1, *}$, N. Spalinger ${ }^{1}$, K. E. Hersberger ${ }^{1}$, I. Arnet ${ }^{1}$

${ }^{1}$ Pharmaceutical Care Research Group, University of Basel, Basel, Switzerland

Background and Objective: Non adherence to long-term medication is a common problem that leads to negative outcomes. Drug reminder packaging is a method to support unintentionally non-adherent patients. In 2002, multidrug punch cards were introduced in Swiss community pharmacies, but little is known about opinion and satisfaction of the users, i.e. primary care patients.

Setting and Method: Questions were developed as quantitative interviews; ambulatory users of multidrug punch cards were recruited in community pharmacies in the cantons of Basel-Stadt, BaselLandschaft, Aargau, and Solothurn; interviews were conducted by phone; analysis of the results delivered the next set of questions that will be asked qualitatively during face-to-face in-depth interviews.

Main outcome measures: Opinions measured by 4-point likert scales, multiple choice and visual analogue scale (VAS); statements analysed according to emergent themes and a catalogue of original objectives.

Results: A structured interview guide with 31 questions was applied to 22 patients who were 74.5 years old (median; range 37-96), mostly female $(63.3 \%)$, retired $(95.5 \%)$, and lived independently and alone $(59.1 \%)$. The use of a multidrug punch card had originally been recommended by the pharmacist $(54.5 \%)$, gave great satisfaction $(90.9 \%)$, and the feeling of security $(100 \%)$. The design of the multidrug punch card was irrelevant for $91 \%$ of the subjects. Taking and timing adherence were on average 99.1 and $92.3 \%$, respectively. Pushing out the tablets caused no problem to $63.6 \%$ of the subjects, although $43 \%(n=5)$ of them declared to cut the aluminium foil on the back instead of pushing the tablets through. Difficulties with handling were mentioned by $27.3 \%$. These discordances will be investigated in the second, qualitative part of the study.

Conclusions: Good adherence, satisfaction, and feeling of security with the use of multidrug punch cards are experienced by ambulatory patients. Questions arising from the quantitative analysis will be deeper investigated in qualitative interviews, e.g. the handling. Disclosure of Interest: None Declared.

\section{CP-PC09}

Importance of pharmaceutical and medical community/hospital collaborations to follow new drugs tolerance and use: example of the abiraterone acetate.

M. Bennani', M. Jardin'1, G. Deplanque ${ }^{2}$, Y. Bezie' ${ }^{1}$, S. Le Moulec ${ }^{3}$, H. Beaussier ${ }^{4, *}$

${ }^{1}$ Pharmacy, ${ }^{2}$ Oncology, Groupe Hospitalier Paris Saint Joseph, ${ }^{3}$ Oncology, Hôpital du Val de Grace, ${ }^{4}$ Pharmacy, CRC, Groupe Hospitalier Paris Saint Joseph, Paris, France

Background and Objective: Abiraterone acetate (AA), in combination with prednisone has been approved for the treatment of men with metastatic castration resistant prostate cancer (mCRPC) who have received or not prior chemotherapy containing docetaxel. The efficacy and safety of AA (1000 mg daily tablet dose) and prednisone ( $5 \mathrm{mg}$ twice daily) therapy in patients with $\mathrm{mCRPC}$ is established by the results of two phase 3 multinational controlled trails (COU-AA301 and COU-AA-302). But patients' feelings about their AA therapy, side effects due to AA or prednisone and drug interactions 
among this population remain unclear. In our study, we assessed those parameters in a cohort of patient treated by AA since its availability in community pharmacies.

Setting and Method: During 2 weeks, a hospital pharmacist asked patients by phone call queries undergoing AA treatment followed in the oncology department of Val de Grâce Hospital. The questionnaire of this survey included 2 main parts: 1/description of AA mode of administration (number and time of pill intake, other medication intake to study drug interactions); 2/analysis of patients' medical file to study duration of AA treatment, reasons of its interruption, duration of the chemical castration and therapy (AA, prednisone) tolerance.

Results: 22 patients were included (mean age: 73 years old). 5 patients refused to answer the questionnaire. The treatment was used according to health agency authorization (fasting, $4 \times 250 \mathrm{mg}$ tablets daily). Major side effects observed were already described in phase 3 trials (bone pain, asthenia, urinary infection, flush, hypokalemia, hypertension, fluid retention) but one wasn't: the weight gain (32\% in our study) and a significant increase for two patients of +10 and $+30 \mathrm{~kg}$ respectively. 20 minor or moderate interactions between AA and other drugs were detected (6 hypokaliemia, 13 interactions with CYP 2D6 or CYP 3A4). The mean duration of the treatment was 10 months: $90 \%$ discontinued AA because of cancer progression and $10 \%$ of death. No correlation between AA treatment duration and chemical castration duration were observed $(\mathrm{r}=0.18, p=0.13)$.

Conclusions: Our results are concordant with those of the phase 3 trials but whether the therapy is accompanied with a significant weight gain remains to be investigated. The community pharmacist, thanks to his overall vision of the patient treatment, has a major role to follow patient tolerance, to detect drugs interactions and to transmit these informations to patient physician and pharmacist in hospital. Moreover, reinforcement of medication adherence and side effects monitoring in this population could be necessary.

Disclosure of Interest: None Declared.

\section{CP-PC10}

Barriers and successful strategies in fostering medication-taking among COPD patients

\section{F. K. Alhomoud ${ }^{1, *}$, K. Taylor ${ }^{2}$, T. Robertson ${ }^{3}$, F. Smith ${ }^{1}$}

${ }^{1}$ Department of Practice and Policy, ${ }^{2}$ Department of Pharmaceutics, UCL School of Pharmacy, ${ }^{3}$ Pinn Medical Centre, Pinn Medical Centre, London, United Kingdom

Background and Objective: COPD patients are often prescribed more than one inhalation device and complex medication regimens, ${ }^{1}$ with alteration of their drug regimen based on disease progression and seasonal changes which may impose a significant barrier to optimal disease management. The aim of this study was to identify barriers and describe strategies employed by patients to facilitate use of medicines. Setting and Method: A cross-sectional study design using semistructured face-to-face interviews was conducted.

Main outcome measures: Identifying barriers and describing approaches for appropriate medicine's usage based upon 46 interviews in patients' homes.

Results: 46 COPD patients (Male $(n=25)$, female $(n=21)$, average age 81.8 years: range $63-100$ years) using multiple inhalation devices were interviewed. Medication adherence was reported to be high in 24 participants $(52 \%)$. However, barriers to medicines use were reported by 22 participants $(48 \%)$ : the most common barriers were forgetfulness and poly-therapy. When missing a dose, participants tend to do the flowing; skip it and continue with their normal schedule $(n=17)$, take the missing dose as soon as recalled $(n=3)$, or doubling the next dose $(n=2)$. Although some participants reported having difficulties in remembering to take their medicines due to advanced age $(n=9)$, cognitive impairment $(n=3)$, or poly therapy $(n=2)$, many referred to their medication-taking behaviour as a habit due to the long-term use, whereas, others had developed strategies to foster medication-taking. The most commonly used strategies were: storing medicines with items that were associated with habitual behaviour (e.g. mobile phones) or in a conspicuous location (e.g. kitchen counter) which is visited often $(n=9)$, making inhalers part of the daily routine $(n=8)$ (e.g. linking to mealtimes), setting a visual or auditory reminder $(n=3)$ (e.g. sticky notes, cell phone alarms), using a calendar/compliance aids $(n=2)$ and getting a person to remind them to take their medicines $(n=2)$. These strategies were perceived as successful and efficient in promoting medicines, by those with high self-reported adherence.

Conclusions: There is no gold strategy for fostering medicationtaking. However, some strategies seemed to work out better than others. Therefore, understanding patients' medication-taking skills and problem-solving strategies may help in maximizing medicine use and optimal management.

Disclosure of Interest: None Declared.

\section{CP-PC11}

Missed drug therapy alerts as a consequence of incomplete electronic patient records in Dutch community pharmacy

A. Floor-Schreudering ${ }^{1,2, *}$, M. Heringa ${ }^{1}$, H. Buurma ${ }^{1}$, M. Bouvy ${ }^{1,3}$, P. De Smet ${ }^{2}$

${ }^{1}$ SIR Institute for Pharmacy Practice and Policy, Leiden, ${ }^{2}$ Departments of Clinical Pharmacy and IQ Healthcare, Radboud University Nijmegen Medical Centre, Nijmegen, ${ }^{3}$ Division Pharmacoepidemiology \& Clinical Pharmacology, Utrecht Institute for Pharmaceutical Sciences (UIPS), Utrecht University, Utrecht, Netherlands

Background and Objective: Complete and up-to-date medical and pharmaceutical information in the electronic patient record (EPR) is a prerequisite for comprehensive risk management in community pharmacy. Our aim was to analyse which information is missing in the EPR and which drug therapy alerts will therefore fail to appear. Setting and Method: Pharmacy master students selected patients who were dispensed a prescription drug and enlisted for $>3$ months in the participating pharmacies. The patients received a questionnaire in which they were asked to verify their medication history including contra-indications and intolerabilities, and to provide additional relevant patient information. For each enrolled patient, the students collected all relevant information from the EPR.

Main outcome measures: Self-reported data from the patient were compared with data retrieved from the EPR. Missed information in the EPR was evaluated based on national professional guidelines.

Results: Questionnaires were received from 442 of 660 selected patients. Prescription drugs were missing in the EPR of $14 \%$ of the 442 patients, non-prescription drugs in $44 \%$, diseases in $83 \%$ and intolerabilities in $16 \%$. In over one third of patients drug therapy alerts failed to appear due to missing information: drug-disease interactions in $34 \%$ of the patients, duplicate medications in $4 \%$, drug-drug interactions in $4 \%$, drug intolerabilities in $2 \%$. Appropriate management did not take place in 375 drug-disease interactions and 27 drug-drug interactions.

Conclusions: Relevant patient information was frequently missing in the EPRs. The non-appearance of drug therapy alerts may have had clinical consequences for patients. Pharmacists should ensure that EPRs are complete and up-to-date.

Disclosure of Interest: None Declared. 


\section{CP-PC12}

The role of point-of-care testing in identification of drug-related problems in a community pharmacy

\section{Balazova ${ }^{1, *}$, M. Grznarova ${ }^{2}$, M. Kuzelova ${ }^{1}$}

${ }^{1}$ Department of Pharmacology and Toxicology, ${ }^{2}$ Faculty's Pharmacy, Faculty of Pharmacy, Comenius University, Bratislava, Slovakia

Background and Objective: Point-of-care testing is one of the clinical activities that pharmacists in community pharmacies in Slovakia are eligible to provide for their patients as a part of their professional practice. The aim was to evaluate drug-related problems (DRPs) identified during these examinations.

Setting and Method: The community pharmacy of Faculty of Pharmacy in Bratislava between 11/2011 and 11/2012. Point-of-care testing provided to patients interested in the study. Testing was followed by a medication review and consultation. DRPs were classified according to PCNE Classification for DRPs V 6.2.

Main outcome measures: Number of detected DRPs and their character.

Results: After an initial point-of-care testing, 78 (88.6\% of 88 ) patients (mean age 64.6 years) showed up for one or more check-up appointments, leading to 169 visits in total. After the first testing, we found that $65(73.9 \%)$ patients had higher than optimal $(>5.0 \mathrm{mmol} / \mathrm{l})$ cholesterol levels, while $9(13.8 \%)$ of these patients were on lipidlowering therapy and $9(10.3 \%)$ patients had the fasting glucose level above the normal range $(>6.1 \mathrm{mmol} / \mathrm{l})$. Blood pressure above $140 \mathrm{mmHg}$ (systolic) and/or $90 \mathrm{mmHg}$ (diastolic) was measured in 35 $(39.8 \%)$ patients. High and very high total cardiovascular risk $(>10 \%$ according to SCORE) was assessed in $29(33.0 \%)$ patients.

We identified 53 DRPs, mostly related to lipid-lowering therapy in $40(75.5 \%)$ cases of the problems. The most common DRP identified in $24(45.3 \%)$ cases was not achieving the optimal outcomes of drug treatment, classified as P1.2. In $12(22.6 \%)$ cases there was a possible DRP of untreated indication (P1.4). Lack of effect of drug treatment (P1.1) was identified in $9(17.0 \%)$ cases, all of them related to lipid-lowering treatment. This problem was caused by not taking prescribed drugs because of fear of possible adverse reactions. Adverse events were detected in $8(15.1 \%)$ cases, $6(11.3 \%)$ of them as non-allergic, namely muscle pain during taking statins.

Conclusions: This work points out that involvement of community pharmacists in providing point-of-care testing together with patient consultation may lead to detection of DRPs.

This work was supported by Grant FaF UK/6/2013.

Disclosure of Interest: None Declared.

\section{CP-PC13}

Not all commercially available splitting devices are sufficiently accurate and precise to use in clinical practice

M. Doeve ${ }^{1}$, B. Van Den Bemt ${ }^{2, *}$, D. Van Riet-Dales ${ }^{1}$, A. Nicia ${ }^{1}$, C. Oussoren $^{3}$, K. Notenboom ${ }^{4}$, Y. Hekster ${ }^{1}$

${ }^{1}$ Medicines Evaluation Board in the Netherlands, Utrecht, ${ }^{2}$ Department of Pharmacy, SINT MAARTENSKLINIEK, Nijmegen, ${ }^{3}$ Faculty of Science, Utrecht Institute for Pharmaceutical Science, Utrecht, ${ }^{4}$ National Institute for Public Health and the Environment, Bilthoven, Netherlands

Background and Objective: This study aims to evaluate the accuracy and precision of commercially available tablet splitters in the Netherlands to subdivide tablets into equal doses and to compare the results with tablets broken by hand and with a kitchen knife.
Setting and Method: Hundred paracetamol tablets were split 3 times with 7 splitting devices: (1) the Lifetime splitter, (2) PillTool splitter, (3) Pilomat splitter, (4) Healthcarelogistics splitter (HCL), (5) PillAid splitter, (6) Fit\&Healthy splitter, and finally (7) a Kitchen knife (Blokker warehouse) The weight of the right and left side of splitted tablets was determined and recalculated into the dose. One hundred tablets were also broken by hand.

Results: Below table depicts the average dose $(95 \%>\mathrm{CI})$ and accuracy (RAS) of each part of the tablets.

\begin{tabular}{llllr}
\hline $\begin{array}{l}\text { Splitting } \\
\text { device }\end{array}$ & Left & Right & \multicolumn{1}{l}{ Left } & \multicolumn{1}{l}{ Right } \\
\hline Lifetime 1 & $312(278-347)$ & $172(123-222)$ & $125(5.7)$ & $69(15)$ \\
Lifetime 2 & $289(255-323)$ & $196(146-245)$ & $116(6.1)$ & $78(13)$ \\
Lifetime 3 & $207(171-242)$ & $283(246-320)$ & $83(8.8)$ & $113(6.7)$ \\
PillTool 1 & $252(225-279)$ & $245(220-271)$ & $101(5.4)$ & $98(5.2)$ \\
PillTool 2 & $248(218-278)$ & $251(224-277)$ & $99(6.2)$ & $100(5.4)$ \\
PillTool 3 & $252(227-277)$ & $247(223-271)$ & $101(5.0)$ & $99(5.0)$ \\
Knife 1 & $235(165-304)$ & $251(192-309)$ & $94(15)$ & $100(12)$ \\
Knife 2 & $236(147-325)$ & $246(156-336)$ & $94(19)$ & $98(19)$ \\
Knife 3 & $231(164-299)$ & $262(199-326)$ & $93(15)$ & $105(12)$ \\
Hand & $245(230-259)$ & $257(243-270)$ & $98(3.0)$ & $103(2.7)$ \\
\hline
\end{tabular}

Scored tablets should comply with the European Pharmacopoeia ( $\mathrm{Ph}$. Eur.) to ensure accurate breaking. The test requires that 30 tablets are broken by hand, one part of each tablet randomly selected and weighed. All 30 parts should be $75-125 \%$ and no more than one outside $85-115 \%$ of the average tablet mass. This paracetamol tablet (RVG 53055) complied with this test (60/60 parts $85-115 \%)$. They also complied when splitting with PillTool (598/600 85\% >115\%; all $75-125 \%$ ), but not with Lifetime $(169 / 600 ; 515 / 600)$ or the kitchen knife (476/600;541/600).

Conclusions: There are relevant differences in the accuracy and precision of six commercially available types of tablet splitters. Fit\&Healthy, LifeTime, PillAid and the kitchen knife are unreliable splitting devices to split paracetamol $500 \mathrm{mg}$ tablets in equal parts. The PillTool splitter, the Healthcarelogistics splitter and the Pilomat splitter are reliable for splitting paracetamol $500 \mathrm{mg}$ tablets in equal parts.

Disclosure of Interest: None Declared.

\section{CP-PC14}

Socio-demographic characteristics and quality of life of elderly patients with hypertension who live in Portugal: a community pharmacy study

\section{E. S. Silva ${ }^{1}$, O. Ribeiro ${ }^{2}$, E. Ponciano ${ }^{3}$, M. Caramona ${ }^{4, *}$ \\ ${ }^{1}$ Community Pharmacy, FARMACIA ROCHA, Coimbra, \\ ${ }^{2}$ Estatistica, Facudade de Medicina, Porto, ${ }^{3}$ IBILI, Facudade de Medicina, ${ }^{4}$ Farmacologia, Faculdade farmacia, Coimbra, Portugal}

Background and Objective: Population aging leads to increased burden of chronic diseases and demand in public health. There is much evidence, that reduction of blood pressure in older adults suffering from moderate hypertension will be accompanied by lower cerebrovascular morbidity and mortality.To describe the socio-demographic 
characteristics and quality of life of elderly patients with hypertension; to correlate the quality of life with clinical factors.

Setting and Method: In a prospective population-based study, 1,039 elderly patients ( 65 years or older) were involved. The patients who visited the community pharmacies were selected. The study was based on a face-to-face interview with a designed diagnostic screening questionnaire. Socio-demographic characteristics, comorbidity factors, and medical history of patients were collected.

Main outcome measures: HRQOL and medical and socio-demographic variables were assessed by questionnaires The data was collected with the use of the following instruments: HRQOL was measured by Hypertension Health Status Inventory, depression by Beck Depression Inventory II, and anxiety by Beck Anxiety Inventory A descriptive analysis, Pearson correlation and t-Student test $(p<0.05)$ were undertaken.

Results: Predominant patient characteristics were: women $(58 \%)$ between the age of 65 and 79, married, 4 years of formal education, retired. It was showed better quality of life scores for men. Older patients perceived their health status as less than younger patients. Having had a stroke was associated with lower quality of life in the physical $(p=0.007)$ and daily $(p<0.001)$ domain. Those who had blood pressure under control scored higher in all domains $(p<0.001)$. Those with only moderate hypertension perceived their quality of life to be more favorable than those who have higher readings. The higher the blood pressure and the more symptoms reported, the lower were quality of life scores. High blood pressure accompanied a patient perception that a number of everyday competencies were adversely affected. Patients do not agree with any degree of certainty about the effects of collateral events associated with the disease upon their lives.

Conclusions: Some of the associations between patient characteristics and HYPER physical and/or emotional component scores were different for men and women. This finding underlines the importance of considering these factors in the management of chronically-ill patients in the community pharmacy. The results suggest that chronically ill women may need more psychological support. There is a need for actions to control hypertension and its associated complications, with the purpose of improving quality of life.

Disclosure of Interest: None Declared.

\section{CP-PC15}

\section{Association between asthma knowledge and asthma control}

I. V. Figueiredo ${ }^{1,2}$, C. C. Bento ${ }^{1}$, O. Leitão Silva ${ }^{1}$, M. T. Rodrigues ${ }^{1}$, R. F. Almeida ${ }^{1}$, M. M. Caramona ${ }^{1,2}$, M. M. Castel-Branco ${ }^{1,2}$, F. Fernandez-Llimos, ${ }^{3,4, *}$

${ }^{1}$ Pharmacology and Pharmaceutical Care, Faculty of Pharmacy, University of Coimbra, ${ }^{2}$ Center for Pharmaceutical Studies, University of Coimbra, Coimbra, ${ }^{3}$ Department of Social Pharmacy, Faculty of Pharmacy, University of Lisbon, ${ }^{4}$ Research Institute for Medicines and Pharmaceutical Sciences (iMed.UL), University of Lisbon, Lisbon, Portugal

Background and Objective: Prevalence of asthma is increasing in industrialised countries. Former classifications of asthma severity are being abandoned in favour of the asthma control concept, meaning "the extent to which asthma manifestations have been reduced or removed by the treatment". Medication adherence has been associated with better asthma control. Patient knowledge about the disease has also been associated with higher medication adherence. However, previous studies failed to identify the association between asthma knowledge and asthma control. Our aim was to identify the potential association between asthma knowledge and two different measures of asthma control.
Setting and Method: Adult patients with medication for asthma visiting four community pharmacies in Central Portugal between Apr and Jun, 2013 were invited to participate. The following questionnaires were applied: Control of Allergic Rhinitis and Asthma Test (CARAT10), Asthma Control Test (ACT), Asthma Knowledge Questionnaire (AKQ).

Main outcome measures: Asthma control: CARAT10 score, ACT; Asthma knowledge: AKQ.

Results: A total of 48 patients, with a mean age of 47 years $(\mathrm{SD}=19.7)$, participated in the study, of which $79.2 \%$ were female. AKQ mean score was $15.5(\mathrm{SD}=2.7)$. The mean CARAT10 score was $18.2(\mathrm{SD}=6.6)$. The mean ACT score was $20.3(\mathrm{SD}=4.3)$. As expected, a significant correlation was found between ACT and CARAT10 scores (Pearson's $p<0.001$ ). When considering control as a categorical variable (ACT $>18$ ), a significant association also appeared with the CARACT10 (Mann-Whitney $p<0.001$ ).

No correlation $(p=0.097)$ between the ACT and AKQ scores appeared. When considering control as a categorical variable, a significant association was identified with AKQ when a 18-point cut-off was used $(p=0.024)$, but not $(p=0.155)$ when the cut-off was 20 . A significant $(p=0.031)$ but very slight correlation $(\mathrm{R}=0.312)$ appeared between CARAT10 and AKQ.

Conclusions: Association between asthma knowledge and asthma control remains in the borderline. Two potential reasons for this situation suggest further research: a lack of sensitivity of knowledge assessment methods, or an actual lack of association between these two variables. Disclosure of Interest: None Declared.

\section{CP-PC16}

\section{Medication adherence in Aruba}

\author{
M. van der Werf ${ }^{1, *}$, R. Angela ${ }^{2}$, G. Fung-A-Fat ${ }^{2}$, B. Kool ${ }^{3}$, \\ J. Bos ${ }^{1}$, H. de Gier ${ }^{1}$
}

${ }^{1}$ Dept. of Pharmacotherapy \& Pharmaceutical Care, University of Groningen, Groningen, Netherlands, ${ }^{2}$ Bureau of Pharmaceutical Affairs, ${ }^{3}$ Ministry of Public Health and Sports, Oranjestad, Aruba

Background and Objective: There is hardly any information on medication adherence in Aruba. Therefore a baseline measurement of medication adherence was performed in Aruba, focusing on patients using oral antidiabetics, antihypertensives and statins.

Setting and Method: Surveys among patients visiting community pharmacies using Medication Adherence Report Scale (MARS) and Beliefs about Medicines Questionnaire (BMQ). In addition database research to measure refill-adherence using pharmacy claims (2011-2012). Main outcome measures: Maximal adherence rate (MARS-score 25), high/low adherence, adherence rates of different groups, differences BMQ answers high/low adherent patients, refill-adherence of complete population (Medication Possession Ratio $\geq 0.8$ : adherent). Results: Surveys In 192 patients, adherence rates for oral antidiabetics, antihypertensives and statins were found as $72.9 \%, 73.9 \%$ and $65.8 \%$ respectively. Patients $>45$ years had a higher mean MARS-score than patients $<45$ years $(p<0.05)$. High adherent patients where older $(p<0.01)$. High adherent patients scored higher on 'necessity' (BMQ). Low adherent patients scored higher on 'harm' (BMQ).

Database Refill-adherence for oral antidiabetics was $74 \%$ (2011, $\mathrm{n}=3,681)$ and $73 \%(2012, \mathrm{n}=3,885)$; for diuretics $79 \%(2011$, $\mathrm{n}=811)$ and $80 \%(2012, \mathrm{n}=819)$; for calcium-antagonists $82 \%$ (2011, $\mathrm{n}=2072 / 2012, \mathrm{n}=2,262)$; for beta blockers $83 \%$ (2011, $\mathrm{n}=2,431 / 2012, \mathrm{n}=2,698)$; for RAAS inhibitors $81 \%$ (2011, $\mathrm{n}=7,684 / 2012, \mathrm{n}=8,154)$; for statins $64 \%(2011, \mathrm{n}=3,471)$ and $67 \%(2012, \mathrm{n}=3,515)$

Conclusions: Surveys Baseline medication adherence has been determined for antidiabetics, antihypertensives and statins as being 
$69 \%$ on average and will serve as baseline for developing and evaluating future programs aimed at improving adherence rates.

Database Compared to Dutch figures (all groups $\geq 90 \%$ ) (1), refilladherence is lower. In comparison to international figures (51-64\%) (2) refill-adherence is higher.

\section{Literature}

1. Medication adherence monitoring in The Netherlands (Dutch). http://www.therapietrouwmonitor.nl/ophalen-medicatie, viewed June 27th, 2013

2. Cramer JA et al. The significance of compliance and persistence in the treatment of diabetes, hypertension and dyslipidaemia: a review. Int J Clin Pract. 2008; 62(1):76-87

Disclosure of Interest: None Declared.

\section{CP-PC17}

Gastroprotection for ns-NSAID users at risk by community pharmacists

M. Teichert ${ }^{1,2, *}$, F. Griens ${ }^{3}$, E. Buijs ${ }^{4}$, M. Wensing ${ }^{2}$, P. D. Smet ${ }^{1,2}$

${ }^{1}$ Science \& Development, Knmp, The Hague, ${ }^{2}$ Scientific Institute for Quality of Healthcare, Radboud University, Nijmegen, ${ }^{3}$ Foundation of Pharmaceutical Statistics, SFK, ${ }^{4}$ ICT, KNMP, The Hague,

Netherlands

Background and Objective: For users of non-selective nonsteroidal anti-inflammatory drugs (ns-NSAIDs) at increased risk of upper gastrointestinal (UGI) complications, gastroprotective agents (GPAs) are recommended. We evaluated the effectiveness of pharmacists' interventions participating in a program to increase gastroprotection in ns-NSAID users at risk in comparison to controls from remaining Dutch community pharmacies.

Setting and Method: In a comparative cohort study participating community pharmacists were reported their ns-NSAID users of 60 years and above at risk for UGI damage. Participating pharmacists intervened to increase safe drug use in consultation with General Practitioners during 5 months in a selected Intervention Group (IG) by adding GPAs to ns-NSAIDs or ceasing ns-NSAIDs.

Main outcome measures: By nationally collected dispensing data after 6 months the status of ns-NSAID use from the selected IG was compared to a control group (CG) from remaining Dutch community pharmacies of ns-NSAID users at risk at baseline by multivariable logistic regression.

Results: At baseline in all ns-NSAID users at UGI risk concomitant gastroprotective medication was detected in $86 \%$. The effect of an intervention to 468 selected ns-NSAID users at risk in 70 participating pharmacies was compared with 20,482 subjects in 1,672 remaining pharmacies at follow up. From ns- NSAID users with baseline risk, persistent ns-NSAID users above 70 years in the IG had an additional $7 \%$ chance on gastroprotection (OR 0.93, $95 \%$ CI 0.89-0.98) compared to the CG at follow-up. Conclusions: Although the percentage of concomitant gastroprotection in susceptible ns-NSAID users in the Netherlands is already high, still substantial numbers of patients remained at risk for serious upper gastrointestinal side effects. Pharmacists led interventions could substantially improve safe use of ns- NSAIDs.

Disclosure of Interest: None Declared.

\section{CP-PC18}

Anti-NMDA receptor encephalitis: Pharmaceutical care and clinical follow-up in pediatrics

H. De Blander ${ }^{1, *}$, L. Falaschi ${ }^{1}$, R. Clément ${ }^{1}$, M. Postaire ${ }^{1}$, P. Bourget ${ }^{1}$
${ }^{1}$ Department of Clinical Pharmacy, Hôpital Necker-Enfants Malades, Paris, France

Background and Objective: Anti-NMDA receptor encephalitis is an auto-immune neuropsychiatric disease characterized in 2007. No specific clinical tools are currently available to assess the efficacy of immunomodulatory treatment among pediatric population. The main objective was to develop an assessment grid and evaluate tolerance and efficacy of the treatment.

Setting and Method: In this study we retrospectively report pharmaceutical care and symptomatic evolution of all the patients admitted to our hospital between May 2010 and March 2013. We evaluated cut-off treatment efficacy and safety at 6 months and 1 year.

Main outcome measures: We developed an assessment grid adapted to the evaluation of children's behaviour, language, motricity, seizures, contact, automatic movement disorders and memory. Each of these characteristics is rated on a scale from 0 (normal) to 3 (grievous). Results: Eight patients aged 3 to 15 years old, 6 girls and 2 boys were evaluated. All of them were observed 6 and 12 months after the beginning of their follow-up. Eight patients received 1st line treatment with intravenous corticosteroids (methylprednisolone) in a bolus at $30 \mathrm{mg} / \mathrm{kg} / \mathrm{d}$ during 3 days and subsequently per os and immunoglobulins at $1 \mathrm{~g} / \mathrm{kg} / \mathrm{d}$ for 2 days. Six patients received rituximab as a $375 \mathrm{mg} / \mathrm{m}^{2}$ intravenous infusion once weekly for 4 weeks. Insufficient improvement leaded to administration of azathioprine started from $1 \mathrm{mg} / \mathrm{kg} / \mathrm{d}$. Immunoglobulins were well tolerated and no hypersensibility occurred; rituximab caused one case of self-limiting hypersensitivity reaction with stopping infusion. One patient developed hypoxic-ischemic encephalopathy secondary to septic shock 3 days after his last rituximab infusion. Clinical course was favourable after 6 months and 1 year for 6 of 8 patients. Switching to azathioprin in 3rd line leaded to an improvement and healing in one patient and another still remains on treatment to this date.

Conclusions: Duration of time before rituximab treatment was often variable, depending on clinician and patient's condition because of the absence of evidence-based formal guidelines. Evaluating the imputability of a clinical benefit to immunomodulatory treatments remains difficult because of co-prescriptions frequently associated. With the support of a new assessment grid adapted to pediatric population, we further highlighted the efficacy of treatment in children.

Disclosure of Interest: None Declared.

\section{CP-PC19}

Medicine-related problems (MRPs) in South Asian (SA) and Middle Eastern (ME) patients in the UK

\section{F. Alhomoud ${ }^{1, *}$, F. Smith ${ }^{1}$, Z. Aslanpour ${ }^{2}$, S. Dhillon ${ }^{3}$}

${ }^{1}$ Department of Practice and Policy, UCL School of Pharmacy, London, ${ }^{2}$ Department of Pharmacy, ${ }^{3}$ School of Life and Medical Sciences, University of Hertfordshire, Hatfield, United Kingdom

Background and Objective: To characterise and examine MRPs from the perspective of SA and ME patients.

Setting and Method: The study was a cross-sectional study. Patients were from SA and ME origins, aged over 18 and prescribed three or more regular medicines. Patients were identified through previous medicine use reports (MUR), patient medication records (PMR) or when presenting with a prescription. The data were collected in 80 face-to-face semi-structured interviews in seven pharmacies in London using MRPs tool. ${ }^{[1]}$ Interviews were audiotaped, transcribed verbatim and analysed thematically using Gordon's coding frame ${ }^{[1]}$ and Nvivo 10 software. 
Main outcome measures: Identification of MRPs from the SA and ME patients' perspectives.

Results: Participants (61\% male) had mean (SD) age 58 (13.4) years and on a mean (SD) of 8 (4) medicines. Final analysis showed the following types of MRPs that influenced adherence and informed decision making among participants: adverse drug reactions and drug interactions; intentional non-compliance; cognitive, physical and sensory problems and issues with concurrent use of herbal and alternative therapies. Problems with drug prescribing; lack of information; monitoring and review; repeat prescriptions; GP surgery and pharmacy service were also identified. Many problems are common in other groups, however, possible explanatory factors discussed how the cultural and religious beliefs, previous experiences, different expectations and needs, different perceptions and actions regarding the use of medicines, language and communication barriers, poor supervision, lack of knowledge or understanding about use of medicines and healthcare services and underestimating patients' desire for information may contribute to the problems.

Conclusions: This study demonstrated that SA and ME patients have their own problems and needs with both medicine use and service access. It also highlighted the crucial role that patients play in the management of their own illnesses. By uncovering particular problems experienced by these groups, the study can inform healthcare professionals, who may have differing views about the appropriate use of medicines, to support SA and ME patients in the use of their medicines and to work with other healthcare professionals to improve access to healthcare services.

Disclosure of Interest: None Declared.

\section{CP-PC20}

Drug interactions of the new oral anticoagulants

\section{A. Trujillano Ruiz ${ }^{1, *}$, E. Urbieta Sanz ${ }^{1}$, C. Caballero Requejo ${ }^{1}$,} D. Rosagro García ${ }^{1}$, M. D. Huéscar Pascual ${ }^{1}$, J. M. Bernal Montañés ${ }^{1}$

${ }^{1}$ Servicio de Farmacia, Hospital General Universitario Reina Sofía (Murcia), Murcia, Spain

Background and Objective: New antithrombotic drugs known as "new oral anticoagulants" (NOAs) have been recently developed, which have, among their main advantages, fewer drug interactions than the antagonists of vitamin-K, although they don't lack them. The aim of this study was to determine the prevalence of potential drug interactions (PDI) described in the literature for the NOAs and to realize a descriptive analysis of them

Setting and Method: The study was conducted in a health area that serves a population of 196.318 inhabitants, for 6 months (July to December 2012). We included all patients in the area treated with one of three currently marketed NOAs (dabigatran (D), rivaroxaban (R) and apixaban (A)).For all of them, demographic data and the complete treatment patients were prescribed simultaneously to identify PDI with NOAs were recorded. Treatment data were obtained from programs that extract information from the medication prescribed and dispensed in primary care $\left(\mathrm{ADN}^{\circledR}\right.$ y Agora plus $\left.{ }^{\circledR}\right)$ and of the ones managing the medication dispensed in an outpatient hospital or possible admissions $\left(\right.$ SAVAC $\left.^{\circledR}\right)$.We considered PDI all the ones described in the technical data sheet and they were completed by review of the current literature available. The PDI found were classified according to the type of NOA involved, the mechanism and the recommendation indicated. We performed a descriptive statistical analysis in which the qualitative variables were expressed as percentages and the quantitative ones as average and its standard deviation

Results: We included a total of 186 patients treated with NOAs during the study period $(110 \mathrm{D}, 75 \mathrm{R}$ y $1 \mathrm{~A}) .64 \%$ were women and the average age $73.8 \pm 9.9$ years. We registered a total of 329 PDI in $87.6 \%$ of patients with an average per patient of $1.75 \pm 1.3(35.5 \% 1$ interaction, $26.3231 .9 \%$ and more than 2).The $19.5 \%$ were pharmacokinetic and $80.5 \%$ were pharmacodynamics. The prevalence of PDI for D was $82.2 \%(1.91 \pm 1.48 /$ patient) and for $\mathrm{R} 84.6 \%(1.57 \pm 1.08 /$ patient $)$. In 27 interactions $(8.2 \%)$ joint administration was contraindicated, in $256(77.8 \%)$ it was necessary to monitor and in $46(14 \%)$ to reduce the dosage and realize a monitorization. The drug groups involved in the PDI were: $50.5 \%$ NSAIDs, inhibitors of P-glycoprotein (IGP-P) (amiodarone, dronedarone, diltiazem) $19.4 \%$, antiplatelet drugs $17.7 \%$, SSRI/SNRI 8.8, $3.3 \%$ anticoagulants, CYP3A4 inducers (phenobarbital) $0.3 \%$

Conclusions: The prevalence of PDI with NOAs found is greater than expected, basically for pharmacodynamic interactions, being necessary studies to evaluate the clinical relevance of them.

Although in most of the PDI the recommendation is to monitor, we found a significant proportion of absolute contraindication, suggesting the realization of a training intervention of this aspect of treatment

A small group of drugs (IGP-P) are responsible of the pharmacokinetic PDI to which patients treated with NOAs are often exposed allowing, in clinical practice, to simplify the review of this aspect Disclosure of Interest: None Declared.

\section{CP-PC21}

\section{Identification of error rate in using inhalation devices} by asthmatic patients

\section{Mazankova ${ }^{1, *}$, I. Mendelova ${ }^{1}$, J. Šaloun ${ }^{1}$}

${ }^{1}$ Department of Social Pharmacy, Faculty of Pharmacy, University of Veterinary and Pharmaceutical Sciences Brno, Brno, Czech Republic

Background and Objective: Asthma is a common chronic disease worldwide and affects approximately 300 million persons in the world. Epidemiologic studies have showed that almost $80 \%$ of patients do not have their asthma under control. Therefore, achieving and maintaining asthma control is the major goal of asthma care. Inhaled therapy creates the preferred treatment for patients with asthma and chronic obstructive pulmonary disease (COPD). The aim of this study was to evaluate the error rate in using inhalation devices (ID), and to define type of errors (steps) in each ID during the process of inhalation technique.

Setting and Method: This study had a cross-sectional design and involved a group of 200 asthmatic outpatients from the Department of Allergology, The University Hospital Brno. Patients were consecutively recruited during their regular visits to the outpatient department, from September 2010 to January 2011. Male and female asthma patients aged 16 to 80 years were eligible for inclusion in the study if they met all of the following criteria: 1) diagnosed with asthma for at least 1 year treatment (J459);2) with inhaled therapy for at least 6 months before starting the study; 3) they were ready to fill in questionnaire. Following variables of participants were reviewed: sex, age, year of treatment, frequency of breath difficulties, classification of asthma according to level of control, ID used. Error rate in using ID was assessed by patients using a questionnaire for each of ID, including all necessary steps for performing correct inhalation technique.

Main outcome measures: We analyzed the percentage of patients with correct and incorrect use of ID, the percentage of errors in assessed ID (Metered Dose Inhaler [MDI], Easy-breathe Inhaler, Diskus, Turbuhaler, Easyhaler, Aerolizer), error rate for each ID separately, generally error rate for all ID, and error rate in concrete steps during the process of inhalation technique. 
Results: About two-thirds of participants $(68.0 \%)$ were women, median age was 32 years. The most frequent occurrence of breath difficulties was more than once a month $(34.0 \%)$, almost half of participants $(49.5 \%)$ had their asthma under control. The most often used ID was Aerosol (51.5\%), followed by Turbuhaler (40.0\%) and Discus $(33.0 \%)$. Incorrect inhalation technique at least in one step was observed in all assessed ID, most frequent in Turbuhaler $(52.0 \%$ of respondents) and Aerosol (40.0\% of respondents). The highest error rate were found in the following steps of inhalation technique: second dose after 1-2 min break (39.1\%), breath out slowly $(24.5 \%)$, rinse mouth $(15.9 \%)$, and remove from mouth $(10.5 \%)$.

Conclusions: Proper inhalation technique of used ID by asthmatic patients is limited. Generally the most frequent error rate is not connected with a direct manipulation with used ID, but more likely with the self technique of carrying out the inhalation. There is a big space for pharmacist intervention with asthmatic patient in a pharmacy, how to improve the patient's knowledge in a proper inhalation technique of inhalation device.

Disclosure of Interest: None Declared.

\section{CP-PC22}

A qualitative study of antibiotic prescribing practices in Long Term Care Facilities using the Theoretical Domains Framework.

\section{A. Fleming ${ }^{1, *}$, S. Byrne' ${ }^{2}$, S. Cullinan ${ }^{2}$, C. Bradley ${ }^{3}$}

${ }^{1}$ Department of Epidemiology \& Public Health and School of Pharmacy, ${ }^{2}$ School of Pharmacy, ${ }^{3}$ Department of General Practice, University College Cork, Cork, Ireland

Background and Objective: Antibiotic prescribing in Irish Long Term Care Facilities (LTCF) has been found to be higher than the European average in recent point prevalence studies. In order to investigate the reasons behind this the aimed to explore the opinions and experiences of health care professionals in LTCF regarding the use of antibiotics in the LTCF context.

Setting and Method: Semi-structured interviews were conducted with 9 community pharmacists, 14 nurses, 14 general practitioners and 4 Consultants who work in or with Irish LTCF. The sampling strategy was convenience sampling with maximum variation. Participants were recruited from rural and urban locations. The analysis was conducted using content analysis and the resulting themes were coded into the Theoretical Domains Framework (TDF) in order to target areas for interventions for future antibiotic prescribing.

Main outcome measures: To identify the factors influencing the management of infection and prescribing of antibiotics in LTCF.

To identify the most relevant domains in the TDF in order to make recommendations for future antimicrobial stewardship strategies.

Results: Beliefs about Knowledge, Environmental context, Memory, attention and decision making processes, and Social Influences were the key areas identified in the analysis. Specific beliefs regarding the importance of decision making autonomy when managing patients with infection, and the lack of structured communication processes, were commonly mentioned. Many strategies for improving antibiotic prescribing in LTCF were suggested such as the need for regular audit, education and multidisciplinary team coherence in antimicrobial stewardship.

Conclusions: This study is the first to evaluate antibiotic prescribing behaviour in LTCF using a behavioural change theory, the TDF. Areas for future interventions include improving knowledge around diagnostic tests and guidelines for antibiotic prescribing, encouraging clear and effective communication of information between professionals, and the importance of appropriate patient assessment.

Disclosure of Interest: None Declared.

\section{CP-PC23}

The use of multi-compartment compliance aids at a population level: a feasibility study

Y. Aguas' ${ }^{1}$, A. I. Nieto' ${ }^{2}$ I. Delgado ${ }^{2}$, A. Garcia Pineda ${ }^{2}$, B. Fernández-Ruano ${ }^{2}$, J. M. Lopez de Lerma ${ }^{3}$, F. Fernandez-Llimos ${ }^{4,5, *}$

${ }^{1}$ Drug Information Center, Badajoz Pharmacists Association,

${ }^{2}$ AIPaFaC Practice-Based Research Network, ${ }^{3}$ Eurosof2000, Badajoz, Spain, ${ }^{4}$ Department of Social Pharmacy, Faculty of Pharmacy,

University of Lisbon, ${ }^{5}$ Research Institute for Medicines and Pharmaceutical Sciences (iMed-UL), University of Lisbon, Lisbon, Portugal

Background and Objective: Multi-compartment compliance aids (MCA) have been advocated as a way to improve medication adherence in patients under polypharmacy. However, their association with improved patient health outcomes has not been sufficiently demonstrated. MCAs are useful only for solid oral pharmaceutical forms. Thus, their practical utility for a real-life vast population has to be explored. We aimed to assess the feasibility of using MCAs in a vast population of medicines users.

Setting and Method: A cross-sectional study was performed using the patients' medication records existing on 31-Dec-2012 in all community pharmacies that are members of the $\mathrm{AIPaFaC}$ practice based research network (PBRN). Patients not visiting the pharmacy in the previous 60 days were excluded from the analysis. Preparing an MCA was considered as 'feasible' if the patient used only solid oral pharmaceutical forms.

Main outcome measures: Feasibility of the use of a MCA for all the medicines used by patients.

Results: Out of the 117,885 patients registered at the AIPaFaC PBRN, 34,441 visited the pharmacies in the previous 60 days, with an average age of 50.0 years-old $(\mathrm{SD}=23.1)$, and being $58.2 \%$ females. Patients used 2.44 medicines $(\mathrm{SD}=2.9)$, being 1.97 $(\mathrm{SD}=2.46)$ solid oral pharmaceutical forms.

MCAs were feasible for $70.3 \%$ of the patients. Patients that could not use MCA were significantly (Mann-Whitney $p<0.001$ ) older [can use 48.5 years; $(S D=21.8)$; cannot use 53.4 years $(S D=25.6)]$; used significantly (MW $p<0.001)$ more medicines [can $1.5(\mathrm{SD}=2.0)$; cannot $4.7(\mathrm{SD}=3.4)$ ]; used significantly (MW $p<0.001)$ more solid oral pharmaceutical forms [can $1.5 \quad(\mathrm{SD}=2.0)$; cannot 3.1 $(\mathrm{SD}=3.0)]$; and used significantly (Chi square $p<0.001)$ more medicines of narrow therapeutic index [can $4.8 \%$; cannot $8.9 \%$ ].

Conclusions: Although a majority of patients using medicines may apparently benefit from a MCA, those patients who deserve a compliance aid the most are patients with lower likelihood of having all their medication into the device.

Disclosure of Interest: None Declared.

\section{CP-PC24}

What factors may influence success of Community Pharmacy Stop Smoking Services?

K. Mortensen ${ }^{1,2,3, *}$, M. Kinnear ${ }^{1,2,3}$, A. Muir ${ }^{2}$

${ }^{1}$ NHS Lothian Pharmacy Service, Western General Hospital, Edinburgh, ${ }^{2}$ Institute of Pharmacy and Biomedical Sciences, University of Strathclyde, Glasgow, United Kingdom, ${ }^{3}$ Department of Pharmacy, University of Tromso, Tromso, Norway

Background and Objective: To obtain a clearer understanding of the factors which may impact on the varying success rates of NHS Community Pharmacy Stop Smoking Services. 
Setting and Method: A questionnaire survey of 182 pharmacies and a national database of success rates and client demographics within one health board area were used to identify factors.

Main outcome measures: Questionnaire parameters included client recruitment methods; staff training; number, type and continuity of staff; use of consultation room; approach used; use of appointments; use of carbon monoxide monitoring and perceived outcomes from repeated quit attempts. Database parameters included gender, age and employment status. Arbitrary pharmacy categories based on their success rates were used to identify potential predictors of success.

Results: During 2012, a median (interquartile range (IQR)) of 19 (6-45) quit attempts per pharmacy was recorded and the IQR for quit rates (self-reported at 4 weeks) was $25.8 \%$ to $55.1 \%$. Questionnaire responses $(n=81)$, suggested the consulting room was used commonly for initial consultations but less frequently for follow-up consultations. Not all pharmacy staff accessed the NHS smoking cessation training, some utilised sponsored training and employment training, some had only one-off training. A higher proportion $(p<0.001)$ of responders with a success rate $\geq 41 \%$ and with client quit attempts $\geq 10$ had clients over 45 years of age compared to responders with a success rate $<41 \%$ and with client quit attempts $\geq 10$. Using the same arbitrary groups there were differences in the proportions of males and females $(p=0.045)$ and employed clients and non-employed clients $(p<0.001)$ having a successful quit attempt. Responses did not suggest other potentially influencing factors on success rates. Respondents requested to receive feedback on their success rates.

Conclusions: Gender, age, employment status of clients and use of the consultation room in community pharmacies are potential factors to test for association with successful smoking cessation outcomes. Feedback of success rates may motivate pharmacies. Defining levels of competence to deliver the service may inform training needs and improve consistency in standard of service delivery.

Disclosure of Interest: None Declared.

\section{CP-PC25}

Medication follow-up at community pharmacy to improve cardiovascular risk

M. Abreu', M. M. Castel-Branco ${ }^{1,2}$, F. Fernandez-Llimos ${ }^{3,4, *}$, M. M. Caramona ${ }^{1,2}$, I. V. Figueiredo ${ }^{1,2}$

${ }^{1}$ Pharmacology and Pharmaceutical Care,, Faculty of Pharmacy, University of Coimbra, ${ }^{2}$ Center for Pharmaceutical Studies, University of Coimbra, Coimbra, ${ }^{3}$ Department of Social Pharmacy, Faculty of Pharmacy, University of Lisbon, ${ }^{4}$ Research Institute for Medicines and Pharmaceutical Sciences (iMed.UL), University of Lisbon, Lisbon, Portugal

Background and Objective: Cardiovascular diseases are the leading cause of death in Portugal, alike what is verified in the remaining western countries. Community pharmacy services have demonstrated improving components of cardiovascular risk (CVR). Medication follow-up consists of a systematic assessment of the patient's medication profile aiming to identify medication negative clinical outcomes, and subsequently performing interventions directed at the patient or the physician. This study aimed to assess the impact of medication follow-up performed in a community pharmacy on cardiovascular risk.

Setting and Method: Randomized controlled study conducted in a community pharmacy in Portugal Mainland. Patients aged between 30 and 65 years-old, under CVR primary prophylaxis were randomly allocated to the intervention group and received medication follow-up service during 6 months, or to the control group receiving usual care. The risk quantification was performed using the SCORE risk scale.
Main outcome measures: Cardiovascular risk measured with the SCORE risk scale.

Results: A total of 23 patients (13 intervention, 10 control) were enrolled in the study. There were no differences between the two groups with regards to basal CVR biomarkers (Body mass, smoking habit, fasting glucose, systolic \& diastolic blood pressure (BP)). No differences existed in a pre-post comparison for any of the components of the CVR in the control group (McNemar $p>0.05)$ and the only component with a pre-post difference in the intervention group was the systolic BP (McNemar $p=0.028$ ). However, when aggregating the components into the CVR using the SCORE, a significant difference appeared in the pre-post analysis in the intervention groups $(\mathrm{McNemar} p=0.046)$ whereas no difference existed in the control (McNemar $p=1.000$ ). Additionally, significant difference in CVR was found between intervention and control groups at the end of the study (Mann-Whitney $p=0.041$.

Conclusions: Medication follow-up performed in community pharmacies could improve cardiovascular risk in patients under primary prophylaxis.

Disclosure of Interest: None Declared.

\section{CP-PC26}

Immunoglobulin self-infusion: an interprofessional drug therapy management program

\section{A. Bourdin ${ }^{1, *}$, J. Berger ${ }^{2}$, O. Bugnon ${ }^{2}$}

${ }^{1}$ Community pharmacy, Department of Ambulatory Care \& Community Medicine, University of Lausanne, Lausanne, ${ }^{2}$ Community pharmacy, Department of Ambulatory Care \& Community Medicine/Community pharmacy, School of pharmaceutical sciences, University of Geneva, University of Lausanne, Geneva/Lausanne, Switzerland

Background and Objective: Patients suffering from primary immunodeficiency diseases are treated with immunoglobulin (Ig) administered in two different ways: 1 . intravenously in a medical setting 2. subcutaneously by the patient himself. As subcutaneous infusion is usually self-administrated at home, autonomy and quality of life of patients should be increased. However, patients need to be educated and supported over the long term by health care professionals to ensure proper adherence and allow optimal efficacy and safety. Our goal was to develop and implement a structured therapy management program, involving community pharmacists and nurses, for patients self-administering Ig therapy. Results: Program description:

1. Patient's skills to undertake subcutaneous Ig treatment are firstly assessed by pharmacists and nurses on predefined criteria. If possible, the program is initiated at the patient's home.

2. Patients follow a structured educative program scheduled from the first dose-where the administration is made by a nurse and a pharmacist - to the fourth dose-where the administration is made by the patient alone, followed by the pharmacist's call.

3. Periodically, administration under supervision (nurse and pharmacist) is performed for preventing "wrong habits" and answering questions. In addition, semi-structured motivational interviews are performed by pharmacists at refills (every three months).

This program is standardized: e.g. documents describe educative sessions and information to be provided and collected by the pharmacists at each step; motivational interviews are based on predefined questionnaires (e.g. occurrence of technical problems or infections); 
indicators allowing to follow adherence, efficacy and safety are also included.

Conclusions: We implemented a standardized and structured program aiming to ensure optimal therapy management for patients under self-infusion therapy. Initial support (i.e. educative program), is ensured by nurses and pharmacists; person-centered long-term support (i.e. adherence, efficacy, safety) is mainly undertaken by pharmacists in collaboration with physicians and nurses. Currently, three patients are actively involved in this program. The next steps are to develop a network of community pharmacies. In addition, a web platform, as a vector for interprofessional collaboration, will be implemented to facilitate a dynamic monitoring. Clinical results will be presented in the future.

Disclosure of Interest: None Declared.

\section{CP-PC27}

Medication reconciliation at discharge in a surgical ward: what are the needs?

\author{
A.-S. Monfort ${ }^{1}$, M.-C. Chaumais ${ }^{1}$, T. Begué ${ }^{2}$, S. Foucher ${ }^{1, *}$, \\ A. Rieutord ${ }^{1}$, S. Roy ${ }^{1}$ \\ ${ }^{1}$ Pharmacy, ${ }^{2}$ Orthopeadic surgery, Hôpital Antoine Béclère, AP-HP, \\ Clamart, France
}

Background and Objective: Patient's hospital care transition process is associated with high risk of medication errors due to poor communication and inadvertent information loss. Thus, for two years, our hospital pharmacy department conducts medication histories for patient admitted in surgical ward. They allow the continuity of patient's treatments from home to hospital. The objective of our study was to assess whether medication reconciliation (MR) at hospital discharge can, firstly provide useful medication information for a continuous therapeutic care to general practitioner (GP) or physician in long-term care settings (PLTCS), and secondly allows patients to understand their treatments, modifications and monitoring.

Setting and Method: A 5 weeks prospective study has been conducted. All patients admitted in orthopaedic surgical unit, with at least 2 chronic diseases and 3 treatments received MR at discharge. A discharge information leaflet (DIL) reviewing all the treatment's modifications during hospitalization was written by a pharmacy student and validated by the surgeon. This DIL was given to the patient during a "pharmaceutical interview" before the discharge. It was also sent to the GP and the PLTCS if concerned. A satisfaction questionnaire given to the patient and sent to physicians evaluated the DIL and its purpose.

Main outcome measures: The DIL was evaluated on the format, content and readability. Patients have also evaluated its purpose on: better understanding on treatments, monitoring and changes. Physicians have evaluated its effect on: better understanding on therapeutic care, medication information source to write the next prescription.

Results: Thirty patients have been included: $64 \%$ have been transferred to long-term care settings and $20 \%$ went back home. The DIL was given to 18 patients, sent to 20 PLTCS and 30 GP. The response rate was: $60 \%$ for patients, $47 \%$ for PLTCS and $13 \%$ for GP. All responders considered that the format, content and readability of the DIL were satisfactory.

Sixty-eight percent of patients reported that they already know all their treatments. However, they believe that the DIL allows to better understanding their treatments (56\%), monitoring $(67 \%)$ and changes $(78 \%)$. Seventy-five percent and $100 \%$ reported that the DIL helps to better understand the therapeutic care and $88 \%$ and $67 \%$ used it to write their next prescription, for PLTCS and GP respectively. However, although these results were very positive, no conclusion is possible for GPs because of the too low response rate.
Conclusions: Although most patients reported already know their treatments, the DIL seems to be useful to better understanding of them, changes done during hospitalization and follow-up. Our results seem to indicate an interest of the DIL primarily for the PLTCS. Indeed, unlike GPs, they care patients right after the hospital discharge. They need practical information about patients' treatments provided by this DIL. It also allows the cross-setting communication improvement at discharge.

Disclosure of Interest: None Declared.

\section{CP-PC28}

Competence and perception of the role of the pharmacist as a breastfeeding counselor

\section{F. Noirhomme-Renard ${ }^{1}$, R. E. Osorio Lopez ${ }^{1}$, C. Gosset ${ }^{1}$, T. Van Hees ${ }^{2, *}$}

${ }^{1}$ Department of Public Health Sciences, ${ }^{2}$ Department of Clinical Pharmacy, University of Liège, Liège, Belgium

Background and Objective: The World Health Organization and UNICEF recommend exclusive breastfeeding during the first 6 months of life and breastfeeding along with appropriate complementary foods up to 2 years of age or beyond. In Belgium, the opinion of the Superior Health Council on the exclusive sales in pharmacy of infant formula (2008) underlines "the importance of informed advice from a university health professional, educated and trained in health issues and nutrition." The pharmacist, through frequent contact with young parents, can play a role in promoting and facilitating breastfeeding.

This study aims to explore the competence and perception of the role of the pharmacist as a breastfeeding counselor among students in pharmacy.

Setting and Method: Fifty-three final year students in Pharmacy of the University of Liege, Belgium, were interviewed by a selfadministered questionnaire in March 2012.

Main outcome measures: This questionnaire included questions about their perceptions, their knowledge and their experience in breastfeeding, and a scale of attitude toward to infant feeding, the IIFAS (Iowa Infant Feeding Attitude Scale).

Results: $92 \%$ of students think that the pharmacist has an important or very important role as a breastfeeding counselor at different levels (recommendations, medications, infant formula,...), and $77 \%$ believe that he can influence the decisions taken by the mothers, even if the pharmacy does not always appear as a good place to discreetly answer to questions $(41 \%)$.

Although professional and personal experience of students is low, their attitude is rather favorable to breastfeeding according to the IIFAS (score $61.25 \pm 7.17$ ). However, students assess their overall level of knowledge as low to very low on topics such as: the deficiency of breast milk, the stimulation of lactation, the use of breast pump, the breast milk storage, the sterilization of baby bottles...

Conclusions: Future pharmacists believe they have an important role as advisor on breastfeeding, but it is necessary to strengthen their current training curriculum to enable them to properly fulfill, in community pharmacy, this role of advisor towards young parents. Disclosure of Interest: None Declared.

\section{CP-PC29}

Diabetes mellitus care in Qatar: a survey of pharmacists' activities, attitudes and perceived barriers

\section{S. El Hajj ${ }^{1, *}$, M. A. Basri ${ }^{2}$, S. E. Abu yousef ${ }^{2}$}


${ }^{1}$ Clinical Pharmacy and Practice Section, ${ }^{2}$ Qatar University College of Pharmacy, Doha, Qatar

Background and Objective: Diabetes mellitus is recognized as a global major public health issue. It is one of the main causes of ill health and premature mortality in many countries. It increases the risk of cardiovascular diseases and is associated with many microvascular complications. With good management and education many of these complications can be prevented or at least delayed. In Qatar, diabetes affects approximately $17 \%$ of the adult Qatari population and it accounts for $18 \%$ of the total health care expenditure. The emerging diabetes epidemic represents an opportunity for Qatar pharmacists to demonstrate their impact on patient care. The study objectives were to describe the practices and attitudes of Qatar community and ambulatory pharmacists toward diabetes, and to assess their perceived barriers for the provision of diabetes care.

Setting and Method: The study objectives were addressed in a cross sectional survey of community and ambulatory pharmacists in Qatar. A phone call explaining the study was made to all pharmacists. Consenting pharmacists anonymously completed the survey either online or as paper. Data were analyzed using Statistical Package of Social Sciences (SPSS ${ }^{\circledR}$ ) Version 20. The study was exempted from full ethics review by Qatar University institution Review Board.

Main outcome measures: Qatar pharmacists' diabetes related activities, attitudes toward diabetes and perceived barriers for diabetes care provision.

Results: Over a 7-month period, we collected 126 surveys (24\% response rate). More than $70 \%$ of respondents said that they always or often discuss the importance of self testing of blood glucose with diabetic patients. More than $60 \%$ of respondents stated that they always or often counsel about the side effects of oral anti-diabetic drugs. However, only around $50 \%$ indicated that they always or often provide education on the importance of regular screening for nephropathy and retinopathy. Using the diabetes attitude scale-3, most respondents had positive attitudes toward need for special training, psychosocial impact of diabetes, and patient autonomy. The top two perceived barriers for providing diabetes services were lack of time (53\% of respondents) and shortage of personnel (42\%).

Conclusions: Overall, Qatar pharmacists have positive attitudes toward diabetes. Interventions should be implemented to overcome their perceived barriers and to improve their diabetes services.

Disclosure of Interest: None Declared.

\section{Community pharmacy: clinical education}

\section{CP-CE01}

Integration of Clinical Pharmacy course in European undergraduate curricula

\section{K. Ladova ${ }^{1, *}$, A. Alwarafi ${ }^{2}$, J. Vlcek ${ }^{1}$}

${ }^{1}$ Department of Social and Clinical Pharmacy, Faculty of Pharmacy in Hradec Kralove, Charles University in Prague, Hradec Kralove, Czech Republic, ${ }^{2}$ Faculty of Dentistry, Ibb University, Ibb, Yemen

Background and Objective: Clinical pharmacy promotes the rational and appropriate use of drugs and medicinal devices which is part of patient-oriented needs of the current pharmacy professions. The aim of the study was to reflect Clinical Pharmacy course (CPC) in the European pharmacy undergraduate curricula.

Setting and Method: Our questionnaire survey was conducted in the period of April to May 2013. All the European faculties with pharmacy undergraduate study and with available e-mail contacts to persons in departments of clinical pharmacy or (vice)deans were addressed. The questionnaire was divided into 7 parts comprising contact form and questions about CPC eligibility, form of CPC teaching, clinical pharmacy departments and staff, syllabus of CPC, and comments of respondents. The questionnaire was created in a web form. Data were processed by descriptive statistics.

Main outcome measures: Proportional integration and form of teaching of clinical pharmacy in pharmacy undergraduate curricula.

Results: The respond rate was $73(30.7 \%)$ questionnaires out of which 71 questionnaires were analysed. $58(82.9 \%)$ faculties had CPC in their undergraduate study. $49 \mathrm{CPC}$ were in compulsory and 9 in optional form. 3 faculties offered both forms of eligibility. Compulsory CPC was taught 2.57 semesters on average and the most hours (h) per week were taught as lectures (on average $3.35 \mathrm{~h}$ ). The most frequent way of teaching of optional CPC were seminars/ workshops $(3.7 \mathrm{~h})$ and training in hospital/ward of clinical pharmacy (2.1 h). CPC was taught most often by clinical pharmacist. 27 faculties had own department of clinical pharmacy.

Conclusions: Our survey showed that CPC is already integrated in the majority of pharmacy undergraduate curricula in Europe and it devoted more than $2 \mathrm{~h}$ per week in active and passive forms of teaching. As a good message may seems that CPC is taught particularly by clinical pharmacists.

Disclosure of Interest: None Declared.

\section{Hospital pharmacy: pharmaceutical care}

\section{HP-PC20}

Identifying potential drug-drug interactions in the intensive care unit: Source matters!

D. Vanham ${ }^{1, *}$, P. Hantson ${ }^{2}$, X. Wittebole ${ }^{2}$, A. Spinewine ${ }^{3,4}$, B. Sneyers ${ }^{1,4}$

${ }^{1}$ Pharmacy, ${ }^{2}$ Department of intensive Care and Emergency Medicine, Cliniques universitaires Saint-Luc, Bruxelles, ${ }^{3}$ Pharmacy, Centre hospitalier universitaire, Mont-Godinne/Dinant, ${ }^{4}$ Clinical pharmacy research group (CLIP), Université catholique de Louvain, Louvain drug Research, Bruxelles, Belgium

Background and Objective: Intensive care unit (ICU) patients are at risk of developing drug-drug interactions (DDIs). Prevalence and management data on DDIs in ICU patients are scarce. Many sources may be used to find information on potential DDIs (pDDIs) but discrepancies in provided information were described.

We sought to describe prevalence of pDDIs in the ICU, as well as mechanisms involved, documentation, severity and management. The second objective was to compare three sources in terms of severity and clinical management.

Setting and Method: A retrospective point of prevalence study in a 975-bed academic hospital (Cliniques universitaires Saint-Luc). All adult patients staying for at least three days in ICU between May and December 2011 were included. Prescribing data on day three postadmission were collected. A pharmacist screened the data searching for pDDIs using three compendia (Stockley's drug interactions, Micromedex, Epocrates). Information on consequences, mechanisms, documentation, severity, management were retrieved.

Main outcome measures: The primary outcome was prevalence of pDDIs. Our secondary objective was to determine reliability between different sources for detection and management. Concordance was measured using Kappa statistics.

Results: A total of 275 patients were included and $79 \%$ presented at least one pDDI. An average of four pDDIs was found per patient. Overall prevalence of contraindicated or major pDDIs was $18.6 \%$ and $9.1 \%$ were documented in at least one randomized controlled trial, cohort or case control study. Four hundred and four interactions were found in this study, but only $12.9 \%$ were detected by all of the 
three sources used for identification. Most pDDIs were classified as moderate and proposed management was mainly monitoring. The Kappa tests between the Stockley, Micromedex and Epocrates were $0.06,0.03,-0.35$ and $0.32,0.38,0.15$ respectively for detection and management, showing low agreement between sources.

Conclusions: Interactions are frequent in the ICU population. Unfortunately, discrepancies between sources of information reflect the need to use different compendia to identify and manage pDDIs. Disclosure of Interest: None Declared.

\section{Hospital pharmacy: pharmaceutical care}

\section{HP-PC21}

Patient satisfaction with drug reconciliation at discharge from hospital

\section{H. Holm ${ }^{1, *}$, K. K. Viktil ${ }^{2,3}$}

${ }^{1}$ South-East Norway Pharmaceutical Trust, Gjøvik Hospital Pharmacy, Gjøvik, ${ }^{2}$ School of Pharmacy, University of Oslo, ${ }^{3}$ Diakonhjemmet Hospital Pharmacy, Oslo, Norway

Background and Objective: Upon discharge from hospital it is essential that the patients receive understandable and unambiguous information about changes in their drug regimen. The objective was to investigate whether a new template for drug information (based on the IMM model) could increase readability and usefulness for the patients, compared with the standard discharge letter. Further, we aimed to examine the patients' knowledge of the drug changes.

Setting and Method: This pre-post study was performed at the Medical department, Innlandet Hospital Trust Gjøvik, Norway. During a ten-week period patients discharged to their home with at least one drug change in their medication regimen since admission were included. Half of the patients received standard discharge letter (control group, CG), and the other half received the new template (intervention group, IG). Both groups were interviewed by a clinical pharmacist at discharge and by telephone 2-4 weeks later about the discharge form, and their knowledge about the drug changes they were discharged with. Patient satisfaction was measured on a six-point scale, from 1 (low) to 6 (high) for readability and usefulness. Readability was based on 5 selected parameters.

Main outcome measures: The patients' grading of readability and usefulness of the discharge form. Number of patients that could account for their drug changes.

Results: 34 patients included: 16 in the IG (mean age $63,50 \%$ women, mean 6.3 drugs), 18 in the CG (mean age $69,50 \%$ women, mean 6.6 drugs). The five selected parameters for readability ranged from 5.2 to 5.9 in the IG and from 4.8 to 5.0 in the CG. The usefulness of the drug information at discharge was $63 \%$ higher in the IG than in the CG. In the IG $75 \%$ of the patients could account for drug changes at discharge compared to $28 \%$ in the CG. The adherence to the drug changes after discharge was comparable for both groups; however, the knowledge about the indications for the prescriptions was increased in the IG. Conclusions: The new template for drug information was superior to the standard discharge letter with regard to readability and usefulness for the patients, and contributes to better overview of drug changes at discharge.

Disclosure of Interest: None Declared.

\section{HP-PC22}

Error analysis of conciliation at discharge in a surgical service

R. Albarracín García 1,*, C. García-Molina Sáez ${ }^{1}$, E. Urbieta Sanz ${ }^{1}$, I. Fortea Gorbe ${ }^{2}$, M. Madrigal de Torres ${ }^{3}$, M. D. Perez Cárceles ${ }^{4}$
${ }^{1}$ Farmacia, Hospital General Universitario Reina Sofía (Murcia),

${ }^{2}$ Tecnologia de los Alimentos, Universidad Catolica San Antonio de Murcia, ${ }^{3}$ Servicio de Cirugía General, ${ }^{4}$ Medicina Legal, Hospital General Universitario Reina Sofía (Murcia), Murcia, Spain

Background and Objective: Determine and analyze the error rate of conciliation (EC) found in the treatment prescribed at hospital discharge reports in the Service of General Surgery.

Setting and Method: Observational cross-sectional study conducted in a referral area hospital. We included all patients who were discharged for two months by the Service of General Surgery whose ingress was greater than $48 \mathrm{~h}$. In order to determine the error rate of conciliation and characterize them we compared the treatment prescribed by the physician in the discharge report with home famacotherapeutic history (HFT) at admission and treatment during hospitalization. The HFT was performed by a pharmacist by means of a structured interview with the patient/caregiver. Any difference between the medication recorded and the treatment prescribed at discharge report was considered as discrepancy. The discrepancies that needed clarification were evaluated with the physician after discharge of the patient to establish whether they were EC. When a relevant error was identified, the physician responsible remedied it with the patient within $72 \mathrm{~h}$ after discharge. Errors were classified according to the Consensus Document on Terminology on Conciliation developed by the SEFH. Later, the potential severity was assessed using the scale The National Coordinating Council for Medication Error Reporting and Prevention's. The data were recorded and analyzed in SPSS v21.0 database created for this purpose.

Results: We included 211 patients, of whom $52.1 \%$ were women. The average age was $61.2 \pm 16.7$ years. $66.4 \%$ of patients had more than 5 medications in their treatment at hospital discharge, with an average of $6.7 \pm 3.6$ medications per patient. 1,763 total discrepancies occurred in $100 \%$ of patients. Of the total discrepancies $28.7 \%$ were justified (5.9\% intentional suspension of a drug, $5.9 \%$ change in dose, route and schedule and $88 \%$ initiation of new drug). 1,258 EC were found that affected $95.3 \%$ of patients, being the average per patient of $4.95 \pm 3.4$. The most frequent error type was omission of medication $(85.3 \%)$.Other types of errors found were incomplete prescription $(11 \%)$, interaction/duplication $(2.7 \%)$, difference in the dose, route or timing $(0.6 \%)$, unnecessary drug $(0.2 \%)$, difference of medicine $(0.08 \%)$.Regarding the severity of errors $62.8 \%$ would have not reached the patient, $23.4 \%$ would had reached the patient causing harm and $12.6 \%$ would had reached but unlikely to cause harm.

Conclusions: We found a rate of patients with EC at discharge very high.The omission of drugs was significantly more frequent than other errors. The prevalence of errors that would produce damage is important and should alert of the need to improve the process since it is an avoidable harm.

Disclosure of Interest: None Declared.

\section{HP-PC23}

Drug-drug interactions in hospitalized cancer patients: Are they common and how do we know them?

\section{Henri ${ }^{1, *}$, A. Wertz ${ }^{1}$}

${ }^{1}$ Pharmacy, Cliniques Universitaires Saint-Luc, Brussels, Belgium

Background and Objective: Drug-drug interactions (DDIs) are frequent. Hospitalized cancer patients are at high risk for DDIs because they are polymedicated and often frail. Studies evaluating DDIs' knowledge among healthcare professionals find a weak rate of detection of interactions. Although ability to recognize and manage potential deleterious interaction could decrease adverse events (Ko 2008). 
The objectives were firstly to determine the prevalence and the characteristics of DDIs in oncology and hematology patients hospitalized in the Cliniques Universitaires Saint Luc in Brussels. Secondly to assess the DDIs' knowledge among healthcare professionals (clinicians and pharmacists).

Setting and Method: This observational retrospective study analyzed the treatments of 200 patients by searching for potential interactions from two interaction databases: Stockley and Micromedex. Mechanisms of action, severity and evidence levels, as well as the type of management were collected. We then selected a subset of the most heterogeneous DDIs for the knowledge survey. This list was submitted to healthcare professionals in June 2012. They were asked to identify the presence of interactions in 15 drug pairs, describe the clinical effects and the appropriate management without using any reference material. Main outcome measures: The main outcomes of this study were the prevalence of DDIs in the study population and the recognition, clinical effect and management scores of the healthcare professionals. Results: 277 DDIs involving 134 drugs were observed. Patients had a mean of 2.8 DDIs. One third of the interactions was pharmacokinetic and $89 \%$ were moderate to severe. Most of the drugs involved were supportive care drugs (e.g. opioid, benzodiazepine). The principal management strategy was monitoring.

$57 \%$ of the drug pairs were correctly categorized by more than $50 \%$ of the healthcare professionals. Drug pairs recorded in the computerized alert system of the hospital had the best score among the clinicians. The recognition scores were better than the clinical effects and the management scores.

Conclusions: DDIs are common in hospitalized cancer patients (77\%). Most of interactions involve supportive care drugs. Although the severity levels were overall quite severe, the real clinical impact remains unknown.

DDIs' knowledge amongst healthcare professionals could be improved. However, keeping in mind all interactions and management strategies is impossible. Therefore targeting specific populations and drugs and using specific computerized alerts could help to avoid potentially deleterious DDIs.

Disclosure of Interest: None Declared.

\section{HP-PC24}

Appropriateness of prescribing direct oral anticoagulants: a prospective cohort study

A.-S. Larock ${ }^{1, *}$, A. Spinewine ${ }^{1,2}$, J. Douxfils ${ }^{3}$, F. Mullier ${ }^{4,5}$, J.-M. Dogné ${ }^{3}$, C. Chatelain ${ }^{6}$ and Namur Thrombosis and Hemostasis Center (NTHC), Namur Research Institute for LIfe Sciences (NARILIS)

${ }^{1}$ Department of clinical pharmacy, CHU UCL Mont-Godinne-Dinant, Yvoir, ${ }^{2}$ Louvain Drug Research Institute, Université Catholique de Louvain, Brussels, ${ }^{3}$ Department of Pharmacy, University of Namur, Namur, ${ }^{4}$ Hematology laboratory, CHU UCL Mont-Godinne-Dinant, ${ }^{5}$ Department of Pharmacy, University of Namur, ${ }^{6}$ Department of Hematology, CHU UCL Mont-Godinne-Dinant, Yvoir, Belgium

Background and Objective: Direct oral anticoagulants (DOACs) have been developed to address some of the limitations of Vitamin-K antagonists (VKAs) and are claimed to be easier to use. However, deviations from the recommended use have been reported, sometimes leading to serious adverse events. Our objective was to evaluate the appropriateness of prescribing DOACs in real-life clinical practice. Setting and Method: We conducted a prospective cohort study including patients admitted to a 450-bed teaching hospital (Belgium) from April to June 2013, who were taking rivaroxaban (Xarelto ${ }^{\circledR}$ ) or dabigatran etexilate $\left(\operatorname{Pradaxa}{ }^{\circledR}\right)$. A clinical pharmacist interviewed each patient to collect clinical and pharmaceutical data. Additional relevant information was retrieved from the electronic medical record. Appropriateness of prescribing was evaluated using the 10 criteria of the Medication Appropriateness Index. Explicit instructions were added, based on EU summary of the product characteristics and (inter)national guidelines. Due to limited cost: effectiveness data in Belgium, the tenth criteria of the MAI was not used.

Main outcome measures: The primary outcome measure was the prevalence of inappropriate prescribing (patients with $\geq 1$ inappropriate criterion). A secondary objective was to identify the main categories of inappropriateness.

Results: Forty-one patients were evaluated (mean age 74 years, range 51-89); 24 and 17 taking Xarelto ${ }^{\circledR}$ and Pradaxa ${ }^{\circledR}$, respectively). Twenty $(48.8 \%)$ patients had at least one inappropriate rating: 1 inappropriate criterion in $26.8 \%$ and $>1$ in $22 \%$ of patients. The most frequent inappropriate criteria were: wrong dosage $(29.3 \%$, e.g. dose not adapted to renal function); inappropriate choice ( $22 \%$, e.g. prescription of a NOAC in a VKA-naïve patient with severe renal failure or extreme body weight); and inappropriate and unpractical modalities of administration (19.5\%, e.g. once a day administration instead of two, non-adherent patients). Results were overall similar when comparing Pradaxa ${ }^{\circledR}$ and Xarelto ${ }^{\circledR}$, except for the modalities of administration that were more frequently inappropriate with Pradaxa ${ }^{\circledR}$ (30\% of patients). Twenty-one patients $(52.5 \%)$ had experienced one adverse event (bleeding, stroke, hematomas,...) and $12.5 \%$ more than one adverse event.

Conclusions: The quality of prescribing DOACs was suboptimal. Off-label use was frequent and suggests that reinforcing education of health care professionals is needed. This should be a priority for the future.

Disclosure of Interest: None Declared.

\section{HP-PC25}

Clinical significance of interventions made by pharmacists to drug related problems (DRPs)

\section{Buajordet ${ }^{1,2, *}$, K. K. Viktil ${ }^{3}$, J. K. Sund ${ }^{1,2}$, H. S. Blix ${ }^{3,4}$}

${ }^{1}$ Central Norway Hospital Pharmaceutical Trust, Hospital Pharmacy of St. Olav, ${ }^{2}$ Laboratory Medicine, Children's and Womens Health, Norwegian University of Science and Technology, Trondheim, ${ }^{3}$ School of Pharmacy, University of Oslo (UIO), ${ }^{4}$ Lovisenberg Diakonale Hospital, The Norwegian Institute of Public Health (NIPH), Oslo, Norway

Background and Objective: Background The Ministry of Health in Norway has requested an increasing contribution from clinical pharmacists in health care delivery because of serious medication-related issues. The concept of integrated medicines management (IMM) has been approved by the regional health trusts as a model to enhance medication effectiveness and safety.

Objective The objective of this study was to evaluate clinical significance of recommendations proposed by the pharmacists to the physicians to solve drug related problems (DRPs) emerging from drug medication reconciliations and medication reviews.

Setting and Method: Setting and Method Patients admitted to a respiratory and a rheumatology ward at the St. Olavs Hospital, Trondheim University Hospital, Norway, in the period of June to October 2011 were included. All patients using one or more drugs at the time of admission and had DRP's identified by the pharmacist, were included. DRPs were identified through medication reconciliation and medication reviews according to the IMM-model. Each recommendation made by the pharmacists were independently assessed and ranked by en expert team: a physician with a speciality in respiratory diseases or rheumatology, respectively, a clinical pharmacologist and a clinical pharmacist. A six-point scoring system for assessing the clinical significance of pharmacists' recommendations 
according to Hatoum was used, with rankings between 1 (adverse significant) and 6 (extremely significant).

Main outcome measures: The clinical significance of pharmacists' recommendations according to Hatoum.

Results: Results A total of 112 recommendations in 46 patients were assessed. On average four $\mathrm{DRP}^{\prime}$ s per patient were found. $85 \%$ of the recommendations were assessed as somewhat significant or higher ( $\geq$ rank 3). The physicians accepted $71 \%$ of the pharmacists' recommendations

Conclusions: Conclusion The clinical pharmacists' recommendations were clinical significant, and further, the high acceptance rate of the recommendations by the physician shows that the contribution from the pharmacist is important.

Disclosure of Interest: None Declared.

\section{HP-PC26}

Errors associated to the omission of the chronic medication in the discharge report

R. Albarracín García ${ }^{1, *}$, C. García-Molina Sáez ${ }^{1}$, E. Urbieta Sanz ${ }^{1}$, M. Madrigal de Torres ${ }^{1}$, A. Trujillano Ruiz ${ }^{1}$, C. Caballero Requejo ${ }^{1}$

${ }^{1}$ Hospital General Universitario Reina Sofía (Murcia), murcia, Spain

Background and Objective: The discharge reports are an essential document of communication between levels of care. Although it is recommended to describe the full treatment, is a common practice in hospitals to use the term "continue your usual treatment" (CUT) without specifying. The aim of this study was to determine the frequency of discharge reports in which is used the expression CUT comparing a medical and a surgical service, as well as to analyze the possible errors caused by this practice.

Setting and Method: Observational cross-sectional study conducted in an area referral hospital. We included all patients who were discharged for a month in general surgery services (GS) and cardioneumology $(\mathrm{CN})$. We analyzed all discharge reports of patients included in the study, selecting those in which the treatment section contained the words CUT. In order to identify possible errors caused by this practice a pharmacist performed the history of homemedication during admission by means of a structured interview with the patient/caregiver and contrasted it with the treatment prescribed at discharge. After identifying and characterizing errors, they were evaluated with the physician responsible for the patient's discharge to confirm that they were accurate. We analyzed the prevalence of reports that used this formula of prescription, associated errors and the severity of them. To analyze the results we used the Chi square test of person, considering as significant a value of $p<0.05$.

Results: We evaluated the discharge reports of 228 patients $(120 \mathrm{CN}, 108$ GS). 107 reports were selected (46.9\%) containing the indication CUT without specifying, being the ratio significantly higher in GS (61\%) than in $\mathrm{CN}(34 \%)(p=0.01)$. The average number of drugs that patients must have at discharge was $7.6 \pm 3.6$ in GS and $10.56 \pm 4$ in $\mathrm{CN}$. The prevalence of drug error was $75.7 \%$ in GS $(92.2 \%$ by CUT $)$ and $69 \%$ in CN (72.2\% by CUT), without significant differences between the two services. The most frequent error type in GS was drug omission $(90 \%)$ compared to $46.1 \%$ in $\mathrm{CN}(p=0.003)$, while the incomplete prescription was found more in $\mathrm{CN}(47.9 \%)$ than in GS $(5.9 \%)(p=0.000)$. As for the errors by interaction/duplicity supposed $4.04 \%$ in GS the $6.04 \%$ in $\mathrm{CN}$. We considered that errors by omission or incomplete prescription would have not reached the patient, since they will follow the schedule of home treatment and it was established that $4.8 \%$ of errors would have reached the patient possibly causing damage.

Conclusions: Half of the discharge reports indicated to the patient to continue with their usual treatment without specifying it, being much more frequent this practice in surgical services than in the medical ones. Using this prescription formula is clearly associated with a high rate of medication errors, which, although not usually reach the patient, are an indicator of poor quality of care should be improved. Our results demonstrate, furthermore, its ability to cause harmful errors to patients although with a low incidence.

Disclosure of Interest: None Declared.

\section{HP-PC27}

\section{Prescription pattern of romiplostim in a tertiary care hospital}

P. Villarón-Hernández ${ }^{1, *}$, E. Farré-Ayuso ${ }^{1}$, N. Creus-Baró ${ }^{1}$, G. Riu-Villadoms ${ }^{1}$, A. Estefanell-Tejero ${ }^{1}$, J. Ribas-Sala ${ }^{1}$

${ }^{1}$ Farmacia, Hospital Clínic, Barcelona, Spain

Background and Objective: To evaluate the prescription pattern of romiplostim in a tertiary care hospital to identify deviations from the summary of product characteristics. Romiplostim is indicated for adult chronic idiopathic thrombocytopenic purpura (ITP) splenectomised patients who are refractory to other treatments. Romiplostim may be considered as second line treatment for adult non-splenectomised patients where surgery is contra-indicated.

Setting and Method: Retrospective study between January 2009 and June 2012 in patients treated with romiplostim in a tertiary care hospital. Main outcome measures: Demographic data, diagnosis, romiplostim dosage, response (evaluated as platelet count $\geq 50 \times 10^{9} / \mathrm{L}$ ) and other treatments of thrombocytopenia.

Results: Fourteen patients were included: Mean age was 65 (SD: 14.7) years, $71 \%$ were women and mean weight was $71 \mathrm{~kg}$ (SD: 10.9). Eleven patients were diagnosed as ITP, 2 as myelodysplastic syndrome (MDS) and 1 as chronic lymphocytic leukemia (CLL). Mean doses of romiplostim were $2.25(\mu \mathrm{g} / \mathrm{kg}) /$ week (SD: 0.984), $10.73(\mu \mathrm{g} / \mathrm{kg}) /$ week (SD: 2.801$)$ and $1.25(\mu \mathrm{g} / \mathrm{kg}) /$ week as ITP, MDS and CLL treatments, respectively. Dose adjustment of romiplostim in ITP was generally done according to product label recommendations, although variations of $0.5 \mu \mathrm{g} / \mathrm{kg}$ were done in $64 \%$ of patients. Romiplostim response rate was $73 \%$ in ITP patients. No increase in platelet count occurred in SMD. Corticoid were prescribed as first line treatment of ITP in all 11 patients. Eight of the ITP patients had received rituximab (off-label use) before initiating romiplostim. Five of the ITP patients and the CLL diagnosed patient received human normal immunoglobulin when bleeding occurred. Nine of the ITP patients and the CLL diagnosed patient were splenectomised. Two of the ITP patients treated with romiplostim were changed to eltrombopag due to inadecuate response, both of them responded to eltrombopag.

Conclusions: Prescription of romiplostim was done according to the labelled indications except in 4 cases. Despite not being specified in the summary of product characteristics, dose variations of $0.5 \mu \mathrm{g} / \mathrm{kg}$ are often used with the aim of minimizing fluctuations in platelet count. No fixed dose per patient was achieved due to fluctuations in platelet counts. Response rate found in ITP patients was as expected according to the literature.

Disclosure of Interest: None Declared.

\section{HP-PC28}

Effect of a clinical pharmacist intervention on unintentional medication discrepancies after discharge: a prospective cohort study

C. Claeys ${ }^{1}$, C. Senterre ${ }^{2}$, J. Nève ${ }^{1}$, P. M. Tulkens ${ }^{3}$, P. Debusschere ${ }^{4}$ A. Spinewine $\mathrm{S}^{3,5}$,

${ }^{1}$ Faculty of Pharmacy, Laboratory of Pharmaceutical Chemistry, ${ }^{2}$ Ecole de Santé Publique, Centre de Recherches Epidémiologie, 
biostatistique et recherche clinique, Université Libre de Bruxelles, ${ }^{3}$ Louvain Drug Research Institute, Université catholique de Louvain, Brussels, ${ }^{4}$ Faculté de Médecine et de Pharmacie, Statistique médicale, Université de Mons, Mons, ${ }^{5} \mathrm{CHU}$ Mont-Godinne Dinant, Université catholique de Louvain, Yvoir, Belgium

Background and Objective: Hospital discharge is a critical period for continuity of care, especially with regard to medications. The effect of clinical pharmacists' interventions has been mainly evaluated in North-American and Australian settings. We aimed to evaluate the effect of such an intervention on the risk of unintentional medication discrepancies after discharge in a European setting taking advantage of the successful launch of Clinical Pharmacy in several hospitals in our country.

Setting and Method: Prospective cohort study conducted between July 2009 and April 2010 in the geriatric and orthopedic wards of 3 hospitals in Belgium. In the first one, clinical pharmacists routinely perform medication reconciliation on admission and at discharge, and provide counseling to patients and healthcare professionals at discharge. No clinical pharmacist was active in the other two hospitals (control group). All patients enrolled were taking $\geq 3$ chronic medications. Each patient was contacted by phone 15 days after discharge to inquire about their current medications.

Main outcome measures: Prevalence and characteristics of unintentional medication discrepancies from the prescription upon discharge, with detection and classification using a validated instrument [1].

Results: From the 341 patients enrolled, 293 could be reached for interview. The prevalence of unintentional medication discrepancies after discharge was $65.0 \%(71 / 109)$ in the clinical pharmacist's intervention group versus $93.5 \%(172 / 184)$ in the control group (OR [95 \% CI]: 0.14 [0.06-0.29]). For patient with $\geq 1$ unintentional discrepancy, the median number of unintentional discrepancies was 2 [range 1-12] in the intervention group versus 6 [range 1-30] in the control group $(p<0.001)$. The most frequent types of discrepancies in the control group were addition (28\%), dosage (22\%) and frequency $(18 \%)$. The most frequent causes were: instructions to patient at transfer incomplete/inaccurate/illegible, instructions between prescribers at transfer incomplete/inaccurate/illegible and medication history incomplete/inaccurate on admission.

Conclusions: Medication reconciliation and patient counseling performed by clinical pharmacists at discharge significantly decreases the risk of medication discrepancies after leaving the hospital. The medication history and reconciliation performed on admission appears also important for minimizing discrepancies after discharge. [1] Claeys C., Nève J., Tulkens P.M., Spinewine A. Content validity and inter-rater reliability of an instrument to characterize unintentional medication discrepancies. Drugs Aging, 29(7), 577-591.

Disclosure of Interest: None Declared.

\section{HP-PC29}

Prescribing errors identified through pharmacy-led medicines reconciliation in a Scottish acute care setting: their frequency, types and clinical significance

\section{E. Past ${ }^{1,2, *}$, S. McGlynn ${ }^{2,3}$, A. Cameron ${ }^{4}$, J. Johnson ${ }^{2}$}

${ }^{1}$ Landesapotheke, Salzburg, Austria, ${ }^{2}$ Strathclyde Institute of Pharmacy and Biomedical Sciences, University of Strathclyde, ${ }^{3}$ Pharmacy department, ${ }^{4}$ Acute medicine department, Glasgow Royal Infirmary, Glasgow, United Kingdom

Background and Objective: Interfaces of care are considered the most vulnerable points for medication errors, a significant source of patient harm. Incomplete or inaccurate handover of medication information often leads to prescribing errors and unintended drug administration or omission. The process of medicines reconciliation can intercept unintended changes to regular prescriptions, and thus improve patient safety.

Setting and Method: This was a prospective observational study of the frequency and type of prescribing errors in a sample of 260 patients admitted acutely under general medicine or cardiology to Glasgow Royal Infirmary, an 850-bedded inner-city teaching hospital in the West of Scotland. Data was collected by a case-note review after the ward pharmacist had completed a formal medicines reconciliation using multiple sources of information. A quantitative study of the potential clinical impact of any prescribing errors was carried out using a questionnaire survey.

Main outcome measures: The main measures were the frequency and type of medication errors identified during the four-week data collection period in 2012. The clinical significance was judged by consensus of pharmacists and doctors using a four-level scale. The number of clinically significant co-morbidities was taken as a crude measure of case complexity, testing for association between case complexity and error rate was done using a generalised linear regression with quasibinomial errors.

Results: At least one medication error was identified in $58.8 \%$ out of 260 patients on admission (95\% CI $52.6 \%>64.8 \%$ ) and in $27.8 \%$ out of 97 patients at discharge (95\% CI $19.4 \%>38.0 \%)$. Omission of a drug on the prescription chart was the most frequent type of error, with dosage errors being the second most frequent. Of the 220 errors detected, $31(14.4 \%)$ were rated as being serious (i.e. having the potential to cause permanent harm to the patient). The probability of an individual patient having at least one serious error on their prescription chart on admission was $8.3 \%$ (95\% CI $5.4 \%>12.5 \%$ ). There was a positive correlation between the error rate and number of co-morbidities (increase in log odds of error 0.1 per co-morbidity, $p=0.004)$

Conclusions: Prescribing errors are commonly made at the time of hospital admission and discharge, and about 1 in 7 of these errors have the potential to cause serious harm to patients. Medicines reconciliation is an important tool for intercepting and correcting these errors, and patient safety could therefore be improved by ensuring that medicines reconciliation is carried out for every patient admitted to hospital. Studies such as this one, which highlight the scale of the problem and the potential for improvement, could be used to convince medical staff of the importance of medicines reconciliation.

Disclosure of Interest: None Declared.

\section{HP-PC30}

Interest of a pharmacist-initiated medication reconciliation in preoperative visit for total hip or knee replacement patients

A. Busumbigabo ${ }^{1}$, C. Staquet ${ }^{2}$, J.-F. Brichant ${ }^{2}$, T. Van Hees ${ }^{1, *}$

${ }^{1}$ Department of Clinical Pharmacy, ${ }^{2}$ Department of Anesthesia and Intensive Care Medicine, CHU of Liège, University of Liège, Liège, Belgium

Background and Objective: Up to $27 \%$ of all hospital prescribing errors are related to incomplete medication history obtained upon admission. Accurate medication reconciliation is an important part of medication safety and a subject of growing interest by health authorities. Few data are available about medication reconciliation at the preoperative visit for total hip replacement (THR) or total knee replacement (TKR).

The objective of this study was to identify significant gaps in the preoperative medication history with the aim of recommending a standardized method of obtaining a comprehensive medication history for orthopaedic surgery patients during the preoperative visit. 
Setting and Method: The CHU of Liège is a teaching hospital of 925 beds. About 225 orthopedic procedures (THR or TKR) are performed each year. All patients are seen preoperatively by an anesthesiologist. During this consultation, the anesthesiologist carries out a medication history.

In a blinded prospective study conducted from December 2012 to June 2013, all elective THR or TKR patients were interviewed by a clinical pharmacist postoperatively. Patients were identified from the daily surgical list.

The medication histories were collected by the clinical pharmacist using a standardized form and compared with the medication information obtained by the anesthesiologist during the preoperative visit, as reported in the medical record.

The clinical relevance of the discrepancies was evaluated by an anesthesiologist after the data collection period.

Main outcome measures: Discrepancies between the two medication lists were classified according to their type, origin, drugs and clinical importance.

Results: A total of 105 patients were successfully enrolled in the study. Population characteristics are $60 \%$ women, $57 \%$ THR, mean age 64 years.

The average number of drugs per patient reported by the anesthesiologist was $5.8 \pm 3.9$ (mean $\pm \mathrm{SD}$ ). The average number of drugs self-reported by the patient to the pharmacist was $5.5 \pm 3.8$, increasing to $8.5 \pm 4.6$ by using specific questions or by contacting the patient's community pharmacist.

Information in the medical chart was incomplete for a total of 484 drugs $(54 \%)$. The discrepancies were: drug omission $61 \%$, treatment schedule omission $14 \%$, dose omission $10 \%$, treatment schedule and dose omission $15 \%$. Omitted drugs were principally painkillers and treatments for osteoporosis.

The clinical relevance of theses discrepancies was evaluated as: severe $11 \%$, moderate $31 \%$, minor $58 \%$.

Conclusions: There are numerous errors in the medication history collected preoperatively. This confirms the importance of reviewing procedures for the collection of medication history. Education of anesthesiologists, use of a dedicated form, active participation of a clinical pharmacist in the preoperative consultation or patient empowerment are possible paths to improvement.

Disclosure of Interest: None Declared.

\section{HP-PC31}

Reconciliation of Chronic Medications in Hospital-Admitted Patients for General and Digestive Surgery

M. Cotaina, ${ }^{1, *}$, M. Juan-Aguilar ${ }^{2}$, P. Modamio', R. Pardo-Sanchez ${ }^{2}$, C. F. Lastra ${ }^{1}$, E. L. Mariño ${ }^{1}$

${ }^{1}$ Clinical Pharmacy and Pharmacotherapy Unit. Department of Pharmacy and Pharmaceutical Technology, Faculty of Pharmacy. University of Barcelona, Barcelona, ${ }^{2}$ Department of Pharmacy, Tortosa Verge de la Cinta Hospital, Tarragona, Spain

Background and Objective: Implementing a continuous model of medication reconciliation is crucial to improve healthcare safety and quality. The objective was to evaluate the results of a pilot study focused on medication reconciliation and thus aid in the development and initiation of a medication reconciliation program.

Setting and Method: The pilot study performed was a prospective study conducted in a district general hospital during 2 months. Selection criteria included patients admitted to the hospital for general and digestive surgery who were previously receiving chronic treatment (at least one medication prior to admission). The medication reconciliation process was performed within the first $48 \mathrm{~h}$ of hospital admission. A thorough comparison of the inpatient medication orders with the record of chronic medication previously prescribed by the patients' primary care physician was performed. Patient interviews were also used to verify accuracy of chronic medication use and other relevant patient information. Post-operative patients were monitored after reinitiating their chronic therapy.

Main outcome measures: Discrepancies were evaluated and classified as either justified or unjustified. Discrepancies not justified by the physician were considered medication reconciliation errors. The degree of potential harm to the patient was also evaluated and recorded according to National Council for Medication Error Reporting and Prevention (NCC MERP). Pharmaceutical intervention was performed by means of oral and/or written communication with the prescribing physician. The physicians' acceptance of the pharmacists' recommendations was recorded.

Results: A total of 33 patients were included (19 female) with an average age of $69 \pm 17$ years. The most common surgical diagnoses were neoplasms $(15 \%)$ and appendicitis (9\%); the most common non-surgical diagnoses were cholecystitis $(18 \%)$ and cholangitis $(9 \%)$. Each patient had an average of $6 \pm 3.5$ chronic medications prescribed prior to admission to the hospital. A total of 141 discrepancies were detected, the average being $4.5 \pm 4.2$ discrepancies per patient. Of these, $122(86.5 \%)$ were justified discrepancies and $19(13.5 \%)$ were unjustified discrepancies (6 missing medication, 2 added medication, 10 different dose/ route/frequency, 1 for different medication). The percentage of prescribing physicians who did not accept pharmacist interventions was $63 \%$. A total of $71.4 \%$ were classified as class B errors and $28.6 \%$ as class $\mathrm{C}$ errors. No life-threatening errors were found.

Conclusions: The results of the study reinforce the importance of implementing medication reconciliation programs and working to improve such programs in order to optimize patient safety. With respect to the degree of acceptance of pharmaceutical interventions, this study demonstrates the importance of creating a multidisciplinary team with shared responsibilities in enhancing patient safety.

Disclosure of Interest: None Declared.

\section{HP-PC32}

Adherence to the ICU Pain, Agitation and Delirium care bundle: Audit of practices in a teaching hospital.

\section{Achen ${ }^{1, *}$, T. Van Hees ${ }^{1}$, P. Damas ${ }^{2}$, B. Sneyers ${ }^{3}$}

${ }^{1}$ Department of Clinical Pharmacy, ${ }^{2}$ Department of General Intensive Care, CHU of Liège, University of Liège, Liège, ${ }^{3}$ Louvain drug research institute (LDRI), Clinical pharmacy research group (CLIP), Université catholique de Louvain (UCL), Louvain, Belgium

Background and Objective: Analgesic and sedative drugs are commonly administered to critically ill patients. Recent recommendations regarding management of Pain, Agitation and Delirium (PAD) in the intensive care unit (ICU) have been published. These suggest metrics to monitor quality of practices in assessment, treatment and prevention of these problems. The aim of our study was to describe current practices and compliance to the proposed PAD bundle in Belgium.

Setting and Method: A prospective 6-week point of prevalence in 3 general ICU wards (a total of 26 beds) in a teaching hospital. Adult patients requiring invasive mechanical ventilation for at least $24 \mathrm{~h}$ were included. Exclusion criteria were pregnancy, cardiopulmonary arrest and palliative care. Baseline demographic data were collected upon admission. The following data were recorded daily: assessment scale, prescription and administration of analgesics and sedatives, daily sedation interruption, physical restraints,...

Main outcome measures: Compliance to predefine metrics.

Results: A total of 34 patients were included (220 patient-days). PAIN- An analgesic was prescribed in $87.7 \%$ of patient-days (193/ 
220). Among these, no pain assessment was documented for $99.5 \%$ of patient-days (192/193). There were $8.7 \pm 6.1$ painful procedures per patient-day but only $1.2 \pm 2.1$ opioids infusions boluses or paracetamol "as needed" administrations per patient-day. -AGITATIONAn intravenous sedative was administered to $54.1 \%$ of patient-days $(119 / 220)$ of which $30.3 \%$ (36/119) for therapeutic coma (i.e. acute respiratory distress syndrome, instable intracranial pressure, refractory epilepsy or use of neuromuscular blocking agents). Among patients for whom sedation monitoring was required (i.e. excluding therapeutic comas) a mean of $1.0 \pm 1.6$ assessments per day was documented and $70.3 \%$ of these (64/91 assessments) measured oversedation (i.e. RASS $=-3$ to -5 , SAS $=1$ to 2 , Ramsay $=4$ to 6 ). Decreasing or stopping infusions accordingly, in within $2 \mathrm{~h}$ of these measurements, was performed in $21.9 \%$ of cases (14/64 assessments). A target score for sedation was prescribed in $34.9 \%$ of patient-days (29/83). Daily sedation/analgesia interruption was performed for $20.5 \%$ of patient-days (17/83). -DELIRIUM- Mean number of delirium evaluations reached $0.3 \pm 3.3$ per day. After excluding therapeutic coma patients, physical restraints were used in $87.5 \%$ of patient-days (161/184) and antipsychotics were administered in 5 patient-days. Among non-pharmacological prevention, mobilization was applied in $81.4 \%$ of patient-days (179/220).

Conclusions: This audit provides several insights for improving PAD management in ICU. Efforts should focus on elaboration of appropriate protocols inspired by the PAD bundle starting with systematic use of assessment scales for an appropriate prescription and administration of drugs. Better consideration of painful procedures and nonpharmacological prevention of delirium must also be undertaken.

Disclosure of Interest: None Declared.

\section{HP-PC33}

Confidentiality: Audit in the pharmacy of a two thousand beds Hospital

\section{E. Cirot ${ }^{1}$, H. Bourgoin ${ }^{1}$, J. Grassin ${ }^{1}$, X. Pourrat ${ }^{1, *}$}

${ }^{1}$ Pharmacy Department, Tours University Hospital, France, Chambray-Les-Tours, France

Background and Objective: Confidentiality is a legal obligation for the health workers. The objective of this study was to check the respect for the confidentiality in the sectors and offices where it must be applied in our pharmacy.

Setting and Method: An observational collection of the confidentiality's faults (possible association between a patient and a pathology) has been made in seven different sectors of the pharmacy: dispensation office, patient's waiting room, distribution room, residents office, clinical studies office, individual dispensation office and secretary. Interviews were organized with the concerned staff (pharmacists, technicians, students...), followed by a general communication about confidentiality. One year later, another collection has been made to evaluate the efficacy of the previous sensitization. Main outcome measures: All these sectors were audited at 4 time slots: 9-12 h (period 1), 12-14 h (period 2), 14-16 h (period 3), 16-18 h (period 4).

Results: Various document types were incriminated: paper prescriptions (45\%), personal bills (19\%), drug administration plans and histories (14\%), patient's folders (paperless or not: $6 \%$ ), clinical studies folders ( $2 \%$ ) and others (14\%). During the first audit, 243 names were read, 28 of which was on computer screens; names for whom the agents were actively working on were not included in the count. The worst period was the second one, the best was the fourth one, with respectively an average of 11.6 and 4.5 names by sector. There is no correlation between the number of persons in a sector and the number of visible names.
On seeing the results, 21 workers have been interviewing about confidentiality, 14 told that they had never been formed. Then, a formation was performed for the entire staff ( 40 workers).

One year later, a new collection was performed, 45 names were read, in only three sectors: dispensation office, residents office and individual dispensation office, respectively 38,2 and 5 names (a decrease of $81.5 \%$ ).

Conclusions: These observations reveal a lack of knowledge and formation of the pharmacy staff in the purview of confidentiality. A sensitization has allowed a significant decrease in confidentiality's faults. This overall awareness became a daily concern, which protects our patients against a leakage of their private information.

Disclosure of Interest: None Declared.

\section{HP-PC34}

Validation of a new Tool for the assessment of Drug Associated Risks (DART)

\section{P. Kaufmann ${ }^{1,2, *}$, N. Mory ${ }^{1}$, K. E. Hersberger ${ }^{1}$, M. L. Lampert ${ }^{1,2}$}

${ }^{1}$ Pharmaceutical Care Research Group, University of Basel, Basel, ${ }^{2}$ Clinical Pharmacy, Kantonsspital Baselland, Bruderholz, Switzerland

Background and Objective: During a patient's hospital stay, his current medication undergoes many changes. This can lead to drug related problems (DRPs) and rehospitalisation. If patients with a high risk for DRPs are identified, they might benefit from optimised individual pharmaceutical care during the hospital stay and upon discharge. A well-directed screening to identify those patients could be an essential contribution to improve patient care. The objective of this study is the development and validation of a screening tool, the Drug Associated Risk Tool (DART), to identify patients at risk for DRPs.

Setting and Method: Risk factors for DRPs, identified in a previous study, provided the basis for the DART. We translated the risk factors into questions that were understandable for patients. We validated the questionnaire concerning feasibility, comprehensibility and acceptability. For the content validity consenting patients filled out the DART and we compared their answers with objective patient data from medical records, laboratory data and data from validated questionnaires. Exclusion criteria were: age $<18$ years, ambulatory or palliative patients; a health status not allowing meaningful communication and no sufficient knowledge of spoken and/or written German to fill out the DART.

Main outcome measures: Feasibility, comprehensibility and acceptability, sensitivity and specificity of the DART.

Results: We created a self-assessment questionnaire containing 18 questions with a total of 35 items. Fifty-one patients (mean age: 67 years [range $20-89$ years], $43 \%$ female) filled out the DART in an average time of $7 \mathrm{~min}, 41$ patients indicated a good level of comprehension. The 18 questions reached an average specificity of 0.96 (range $0.88-1$ ), whereas the average sensitivity was 0.57 (range $0-1$ ). We found an overall correlation between patients' answers and objective data of $70 \%>100 \%$.

Conclusions: The DART is practicable with satisfactory feasibility, comprehensibility and acceptability. In addition, false positive results can be excluded with a high probability due to a consistently high specificity. For more reliable and meaningful conclusions concerning sensitivity, further on-going patient interviews will foster our preliminary findings.

Disclosure of Interest: None Declared. 


\section{HP-PC35}

The influence of promoting good use on the quality of prescriptions

J. Liehn ${ }^{1, *}$, E. Farnier ${ }^{2}$, L. Vadot ${ }^{3}$, B. Leroy ${ }^{1}$, M. Bourdelin ${ }^{4}$, C. Renzullo ${ }^{1}$, H. Bontemps ${ }^{4}$, J. Coutet ${ }^{1}$

${ }^{1}$ Pharmacy, Centre Hospitalier William Morey, Chalon-sur-Saône, ${ }^{2}$ Pharmacy, Centre Hospitalier Pierre Oudot, Bourgoin-Jallieu, ${ }^{3}$ Centre Hospitalier Universitaire, Dijon, ${ }^{4}$ Pharmacy, Hôpital Nord Ouest, Villefranche-sur-Saône, France

Background and Objective: If one considers the adverse events associated with anticoagulants as well as the number of available doses, the different posologies (in relation to age, renal function, bleeding risk factors...) and the drug interactions, NOACs (New oral anticoagulants) should be considered a new class of therapeutic molecules with a high iatrogenic risk. A plan to promote good use by the pharmaceutical team during referencing could limit the risk of this new class of drugs.

The goal of this study is to evaluate the impact of a plan of good use on NOAC prescriptions in a general hospital center.

Setting and Method: A retrospective multicenter study based on an analysis of NOAC prescriptions was performed in two hospital centers: the first center implemented a plan to guarantee good use of NACO including a protocol for good use and training of involved professionals $(\mathrm{CH} 1)$, the second center referenced NACO but had no specific plan to control good use $(\mathrm{CH} 2)$.

All prescriptions including Dabigatran or Rivaroxaban in both hospitals were analyzed for 6 months. Only prescriptions for the following indications were taken into account: prevention of stroke and systemic embolisms in patients with non-valvular atrial fibrillation, treatment of deep venous thrombosis and pulmonary embolism and prevention of recurrence.

Main outcome measures: The main judgement criterion was a comparison of the rate of interventions by pharmacists in each hospital using the Chi square test $(p<0.05)$.

Results: During the study period pharmacists in $\mathrm{CH} 1$ analyzed 151 NOAC prescriptions for a rate of interventions by pharmacists of $13.9 \%$ (17.6 \% for Rivaroxaban and $8.3 \%$ pour Dabigatran).

The pharmacists in $\mathrm{CH} 2$ analyzed 79 NOAC prescriptions. The rate of interventions by pharmacists was $34.2 \%$ (32.4\% for Rivaroxaban and $35.7 \%$ for Dabigatran).

There was a significant difference between the rate of pharmaceutical interventions in the two hospital centers for NOAC prescriptions $(p<0.001)$.

Conclusions: The rate of pharmaceutical interventions was significantly lower in $\mathrm{CH} 1$ for both molecules. Early intervention by the pharmacist before the prescription to promote good usage of these high risk drugs seems to influence the rate of prescription medications errors. Disclosure of Interest: None Declared.

\section{HP-PC36}

Predictive model of error risk of conciliation clinically significant at discharge in a general surgery service

R. Albarracín García ${ }^{1 * *}$, C. García-Molina Sáez ${ }^{1}$, M. Madrigal de Torresi , E. Urbieta Sanz ${ }^{1}$, I. Fortea Gorve², M. Pérez Cárceles ${ }^{3}$

${ }^{1}$ Hospital General Universitario Reina Sofía (Murcia), ${ }^{2}$ UCAM, ${ }^{3} \mathrm{UM}$, Murcia, Spain

Background and Objective: Develop a predictive model for the detection of patients with higher probability of error of conciliation
(EC) that may cause harm to the patient at discharge in a General Surgery service(GSS).

Setting and Method: Observational, cross-sectional study conducted in a referral area hospital. We included all patients who were discharged for two months by the GSS. In total 211 patients were included, with a mean (SD) of 6.66 drugs per patient (SD: 3.57), and a total of 1,405 drugs. Error of conciliation was defined as clinically relevant (ECCR) when they had reached the patient and had caused damage. There were 82 patients with ECCR (average(SD)0.39(0.48)).We studied the association of the presence of ECCR previously established with the following explanatory variables: age, gender, stay, day of discharge, polypharmacy, comorbidity, information sources provided, discharge physician category(resident, assistant), inpatient unit, number of doctors passing visits, helping MIR number, number of discharges, occupancy rate, number of ingress, type of admission, physician age. A predictive model of multivariate logistic regression was applied, introducing the variables mentioned, and obtained the optimal cutoff point for the classification using ROC curve that maximized sensitivity and specificity.

Results: Using a simple logistic regression we identified the risk factors associated with ECCR, and found to be statistically significant: Age less than 45 years is a risk factor compared to those over 45 years(OR $1,964,95 \%$ CI 1,077 to 3,581),and the Type of admission: urgent admission is associated with an increased likelihood of ECCR with respect to the patient's scheduled admission(OR 1.796, $95 \%$ CI 0.996-3.240).The resulting logistic regression model was statistically significant (model $\left.\chi^{2}=5,120, p=0,024\right)$. With a poor setting of the model to the data (Hosmer-Lemeshow test $=$ $0.000, p<0.001)$.The model explains little the ECCR variable behavior (Nagelkerke $\mathrm{R}^{2}=0.033$ ) and do not classify cases correctly(zero sensitivity and specificity of $100 \%$, with an overall correct classification of $61 \%$ ). Only Patient age resulted included in the final equation of the 16 predictors studied $(p=0.026)$, having a greater risk of error of conciliation clinically relevant people under 45 years than those over 45 years $(\mathrm{OR}=2,001,95 \%$ CI 1,085 to 3,691).Using a ROC curve we chose as optimal cutoff value 0.367 with which the selected model (sensitivity: $73.2 \%$, specificity: $58.1 \%)$

Conclusions: It can be assumed that the model fits to the data obtained although only explains $3.3 \%$ of the variability of ECCR, this implies that there is a significant percentage of influence that does not depend on the variables analyzed. It's implied the convenience of generalize the realization of the conciliation at discharge in GSS given the important frequency of patients with ECCR and the difficulty of identifying risk patients.

Disclosure of Interest: None Declared.

\section{HP-PC37}

STOPP/START identification of inappropriate medication for elderly people in Brazil

A. L. P. M. Mori ${ }^{1}$, M. G. F. Lima ${ }^{1}$, A. Yochiy ${ }^{2}$, J. F. S. Carrillo ${ }^{1}$, M. S. P. N. Rossi ${ }^{1}$, P. M. Aguiar ${ }^{1}$, E. L. Dórea ${ }^{3}$, S. Storpirtis ${ }^{1, *}$

${ }^{1}$ Faculty of Pharmaceutical Sciences, University of São Paulo,

${ }^{2}$ Volunteer Pharmacist, ${ }^{3}$ University Hospital of São Paulo, São Paulo, Brazil

Background and Objective: In Health Care the use of multiple medications by the elderly is a concern. There are many potentially inappropriate medications (PIMs) for elderly patients, but methods have been developed to reduce their occurrence. The objective of this study was to determine PIMs and/or absence of appropriate medication in the prescriptions of elderly patients before admission (BA) and 
after hospital discharge (AD), according to the STOPP/START criteria.

Setting and Method: Data on medication were obtained between October 2011 and February 2013 from patients admitted to the medical clinical ward of University Hospital of São Paulo, Brazil. BA data were obtained from patients, family members, medical records and/or prescriptions. AD data were obtained only from prescriptions. Retrospective examination of data by pharmacists was performed using the STOPP/START criteria, which indicate, respectively, the presence of PIM and the absence of medication appropriate in the prescription of elderly people.

Main outcome measures: The level of appropriateness/inappropriateness of prescribed medication used at home by elderly people.

Results: Medication of 245 patients with mean age of $72.8 \pm 8.4$ years, $51.8 \%$ women, was examined. The mean number of medicines used was $5.6 \pm 2.7$ per patient. START criteria revealed 181 and 138 omissions for $\mathrm{BA}$ and $\mathrm{AD}$, respectively, from which the most frequent was the absence of statins for diabetic patients with major cardiovascular risk factors (36.0\% BA and $30.0 \%$ AD). STOPP criteria disclosed 82 and 43 PIMs for $\mathrm{BA}$ and $\mathrm{AD}$, respectively. In this case, glibenclamide for diabetes type 2 was the most frequently found medication, which might increase the risk of prolonged hypoglycemia $(37.0 \%$ $\mathrm{BA}$ and $32.6 \% \mathrm{AD}$ )

Conclusions: The results suggest that STOPP/START criteria can prevent major health risks derived from PIMs and the omission of medication appropriate for the elderly from prescription. It is concluded that STOPP/START should be implemented in the hospital as a routine care of elderly patients.

Disclosure of Interest: None Declared.

\section{HP-PC38}

Homogenization of antiemetic treatments: assessment in pneumology and urodigestive oncology departments for final implementation

E. Duparc ${ }^{1, *}$, C. Borel $^{1}$, C. Monchablon ${ }^{1}$, M. Fontaine-Adam ${ }^{1}$, S. Bota ${ }^{2}$, A. Oden-Gangloff ${ }^{3}$, P. Michel ${ }^{3}$, N. Donnadieu' ${ }^{1}$, A. Coquard ${ }^{1}$, B. Dieu ${ }^{1}$

${ }^{1}$ Pharmacy, ${ }^{2}$ Pneumology, ${ }^{3}$ Urodigestive Oncology, CHU ROUEN, Rouen, France

Background and Objective: Nausea and vomiting, as major side effects of chemotherapies, are subjects to various international recommendations. The inventory of the management of nausea and vomiting, for emetogenic chemotherapies same potential within our institution has shown numerous differences that appeared necessary to homogenize. That is the reason why we developed a standardized protocol that we have evaluated before its final implementation.

Setting and Method: Our antiemetic protocol was based on actual recommendations and technical and practical requirements, and had been validated in Federation of Cancerology. For evaluating the efficacy, the study has been conducted from March to October 2012 through the use in Pneumology and Uro-digestive Oncology departments of a questionnaire for the patients, including automedication use, to be completed during the first course of chemotherapy, and then a self-evaluation application to be completed during the following courses, and through an identification sheet to be completed by the medical doctors.

Results: After 7 months of survey, only 7 out of 21 patients treated by chemotherapy with high emetic potential had presented vomiting and $14 \%$ complained about strong intensity nausea.
For chemotherapies with medium emetic potential, 4 patients out of 28 experienced vomiting, and only $5 \%$ of these patients showed high intensity nausea. For low emetic potential treatments, 4 patients out of 14 presented vomiting, and $5 \%$ of patients experienced important nausea. Among 53 patients who completed the form about automedication, only 3 reported using some other treatments for nausea and vomiting, and 44 patients out of 50 said they had a good compliance to the antiemetic treatment.

Conclusions: This modification has allowed moving from various recommendations, unadapted to hospital practice, to appropriate unified recommendations that could simplify the cares. All data converged to achieve a satisfying result and supported the implementation of this new protocol. This protocol is to be submitted to the regional committee of drugs and medical devices with the goal of homogenization of prescription of antiemetic treatments.

Disclosure of Interest: None Declared.

\section{HP-PC39}

Home medication influence over falls and bone fractures in the elderly: retrospective study of a cohort of 237 patients in an orthopaedic surgery department in France

A. Petitcollin 1,*, J. Druon' ${ }^{2}$, S. Floch' ${ }^{2}$, F. Remérand ${ }^{3}$,

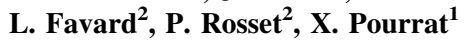

${ }^{1}$ Pharmacie Logipôle, ${ }^{2}$ Orthopaedic Surgery Department, ${ }^{3}$ Intensive Care Department, Hôpital Trousseau, Tours, France

Background and Objective: The elderly are particularly exposed to risks of fall with severe consequences in terms of morbidity and mortality. Several studies have shown the use of certain drug classes and polymedication are risk factors for falling in elderly. We undertook this study to analyze the relationship between home medications and occurrence of falls leading to severe injury and hospitalization in an orthopaedic surgery department (OSD).

Setting and Method: The drug prescriptions of the patients over 65 hospitalized among $2011(\mathrm{n}=815)$ in our OSD were analyzed to identify home medications. Drugs were categorized according to the Anatomical Therapeutic Chemical (ATC) classification system. Patients were splitted in two groups whether or not a fall caused their hospitalization. An age-matched control was randomly associated to each patient who had fallen. We compared the mean number of drugs per patients, the frequency of prescription of drugs per ATC class, and the frequency of exposition of patients to the different drugs classes. According to Laroche's Consensus Panel List [1], we assessed the frequency of prescribing deleterious drug associations and unsuitable medications in patients over 75 and compared them in both groups. Main outcome measures: After age matching, each group included 237 inpatients. $90 \%$ of the fallen had a fracture. Women were majority in the fallen group as compared with controls $(p=.004)$. 2,221 lines of prescription, shared in 44 ATC classes were analyzed. Main number of drugs per patient was 4.60 into the fallen group versus $4.77(p=0.47)$. The proportion of drugs per class was different in N05 (psycholeptics, $p=.006$ ) and N06 (psychoanaleptics, $p=.026)$ classes.

Anxiolytic drugs (N05B) and antidepressants (N06A) were significantly more prescribed in the fallen group $(p=.045$ and .019 respectively). Fallen were more exposed to antidepressants $(p=.031)$. Exposition to anxiolytics was not different in both groups but there was significantly more patients taking two anxiolytics simultaneously among the fallen $(p=.045)$.

Analysis of the medications prescribed to the patients over 75 showed more frequent associations not to be recommended according to Laroche into the fallen group $(p=.004)$. 
Results: This study highlighted that anxiolytics and antidepressants were more frequently found into the home medications of patients who fell, suggesting the prescription of these drugs to people over 65 increases the risks of fall with fracture. Deleterious drug associations were also found in patients over 75 .

Conclusions: A special vigilance is required to identify and withdraw these drug-related falls in favor of less dangerous medications in order to decrease the occurrence of falls in elderly.

[1] Laroche ML et al. Potentially inappropriate medications in the elderly: a French consensus panel list. European Journal of Clinical Pharmacology 2007; 63:725-31.

Disclosure of Interest: None Declared.

\section{HP-PC40}

Characteristics of Renin Angiotensin System Blockers-induced Angioedema in Thailand

T. S. Z. Win ${ }^{1, *}$, S. Nathisuwan ${ }^{1}$, N. Chaiyakunapruk ${ }^{2,3,4,5}$, W. Suwankesawong', K. Tanyasaensook ${ }^{1}$, P. Dilokthornsakul ${ }^{2}$

${ }^{1}$ Department of Pharmacy, Faculty of Pharmacy, Mahidol University, Bangkok, ${ }^{2}$ Center of Pharmaceutical Outcomes Research, Department of Pharmacy Practice, Faculty of Pharmaceutical Sciences, Naresuan University, Phitsanulok, Thailand, ${ }^{3}$ School of Population Health, University of Queensland, Brisbane, Australia, ${ }^{4}$ Discipline of Pharmacy, Monash University Sunway Campus, Kuala Lumpur, Malaysia, ${ }^{5}$ School of Pharmacy, University of Wisconsin-Madison, Wisconsin, United States, ${ }^{6}$ Health Product Vigilance Center (HPVC), Food and Drug Administration, Ministry of Public Health, Nonthaburi, Bangkok, Thailand

Background and Objective: Angioedema is a rare but important side effect of renin-angiotensin system (RAS) blockers. Little information exists regarding this adverse reaction in Asian population. This study aimed to characterize such event in Thai population.

Setting and Method: This study was a retrospective study using the Health Product Vigilance Center database, the national pharmacovigilance database of Thailand established since 1984. Cases were identified using World Health Organization-Adverse Reaction Terms indicating the presence of angioedema with concomitant use of RAS blockers during the period of 1994-2011. For each report, detailed information was obtained and descriptive statistics were performed using STATA 12.0 for all study variables.

Main outcome measures: Patients' characteristics, patterns of RAS blockers usage and patterns of angioedema among Thai population.

Results: A total of 895 cases of RAS-blockers associated angioedema were identified. Mean age was $59.9 \pm 12.8$ years and $66.5 \%$ were female. Most angioedema (48.6\%) occurred during the first week of treatment (median of 5 days). Angiotensin converting enzyme inhibitors $(87.7 \%)$ were the most commonly implicated agents followed by angiotensin receptor blockers $(10.5 \%)$, aldosterone antagonist $(2.1 \%)$ and direct renin inhibitor $(0.2 \%)$. Among these cases, 165 of 895 cases $(18.4 \%)$ were classified as serious events and resulted in hospitalization. The overall case fatality rate was $0.6 \%$. Respiratory disturbance, a serious complication of angioedema, occurred in 46 cases $(5.1 \%)$. These patients tended to be younger $(53.4 \pm 13.9$ vs $60.3 \pm 12.7$ years old; $p=0.002)$ and with higher frequency of allergy history (OR: $2.195 \%$ CI: 0.9-4.3).

Conclusions: Similar to Western population, RAS-blockers associated angioedema in Thai population occurred in mostly elderly female patients. While most reactions were benign, significant proportion of patients may develop serious events which may result in hospitalization or death. Therefore, healthcare professionals should be aware of the seriousness of such event.

Disclosure of Interest: None Declared.

\section{HP-PC41}

Contribution of hospital pharmacy to conduct a ketogenic diet

P. Collart Dutilleul ${ }^{1, *}$, A.-C. Gerout ${ }^{1}$, A. De Saint Martin², M. Fischbach ${ }^{2}$, B. Gourieux ${ }^{1}$

${ }^{1}$ Pharmacy-Sterilization, ${ }^{2}$ Pediatrics, University Hospital of Strasbourg, Strasbourg, France

Background and Objective: The Ketogenic Diet is a therapeutic diet very strict based on a high consumption of fat ( $90 \%$ of energy intake) and low consumption of carbohydrates and proteins. It is proposed in the treatment of drug-resistant epilepsy in children after observing the positive effects of fasting and ketosis in epilepsy. Dietary intakes are calculated in grams by the dietician, but the slightest deviation can let reappear crises. Drugs, especially oral solutions, contain sweet excipients that can disrupt the state of ketosis.

Objectives of our work are to list the sweet exipients found in drugs, and to realize a drug's summary table in terms of their content in sweet excipients with the aim of advise pediatrician and dietician to adapt a ketogenic diet.

Setting and Method: Search of sweet exipients present in drugs. Realisation of a drugs summary table available in the Strasbourg's Pediatric service. Tracking prescriptions drugs of patients on one year.

Main outcome measures: Categorisation according to the excipient and the carbohydrate content of drugs. The prescriptions of the patients were analyzed according to intake in drug's sweet excipients, the need to establish a drug and/or to realise perform compounding. Results: Sweet excipients to avoid are those that turn into glucose in the body. Of 73 drugs presented in oral solutions used at Strasbourg Hospital's Pediatrics Department, only $20 \%$ can be used because they do not contain sugar. The content of sweet excipients changes sometimes depending of the pharmaceutical form and dosage, and may differ between original medicines and generic drugs, and between generic drugs according to the pharmaceutical laboratory. Of 16 prescriptions, the analysis shows that treatments medications prescribed provide sugars in $75 \%$ of cases, that drug should be discontinued or substituted in $62.5 \%$ of cases, and that preparation should be performed in $45 \%$ of cases with suitable excipients (for example: cellulose because it unabsorbed).

Conclusions: Hospital Pharmacy can provide accurate information on the composition of the drug excipients, can offer alternative therapies and can produce compounding adapted to a ketogenic diet. The pharmacist is a focal point for pediatricians and dieticians, who can help to optimize management of patient's treatment.

Disclosure of Interest: None Declared.

\section{HP-PC43}

A cross-sectional survey of antimicrobial strategies in UK hospitals

\section{A. P. Tonna ${ }^{1}$, I. M. Gould ${ }^{2}$, D. Stewart ${ }^{1, *}$}

${ }^{1}$ School of Pharmacy and Life Sciences, Robert Gordon University, ${ }^{2}$ Medical Microbiology, Aberdeen Royal Infirmary, Aberdeen, United Kingdom

Background and Objective: To improve prescribing and reduce antimicrobial resistance, antimicrobial stewardship programmes have been implemented in hospitals, usually led by antimicrobial management teams (AMTs). The objective of this study was to describe the profile and activities of AMTs within hospitals in the UK.

Setting and Method: All hospitals within the UK $(n=836)$; a pre-piloted questionnaire mailed to the 'Director of pharmacy.' 
Non-respondents were mailed up to two reminder questionnaires at two weekly intervals. Results were analysed using SPSS ${ }^{\hat{a}}$ and Minitabs ${ }^{\hat{a}}$.

Main outcome measures: Existence and remit of the AMTs.

Availability of antimicrobial prescribing policies, aims and scope and methods of dissemination.

Monitoring and feedback provided on antimicrobial policy adherence.

Results: Response rate $33 \%(n=273)$.

Completed questionnaires analysed $n=226$. ( $n=47$ incomplete-reasons given include no pharmacy department, already completed by another site).

Responses were largely from English hospitals (70\%); district general hospitals $(57 \%) .82 \%(\mathrm{n}=186)$ of respondents indicated the presence of an AMT within the hospital, with $95 \%$ of these $(n=177)$ reporting an antimicrobial pharmacist as part of the team.

All AMTs $(n=186)$ were involved in development of an antimicrobial policy and almost all $(99 \% \mathrm{n}=184)$ promoted adherence and restricting use of specific antimicrobials $(97 \%, \mathrm{n}=180)$.

$98 \%$ of respondents $(n=222)$ reported the availability of a local antimicrobial prescribing policy within the hospital with this disseminated mainly through the hospital intranet $(98 \% \mathrm{n}=217)$. Adherence to policy was measured mainly through audits measuring the appropriateness of antimicrobial use against the local policy $(76 \% \mathrm{n}=169)$.

Hospitals in England $(p=0.010)$, tertiary care hospitals $(p=0.021)$ and bed capacity $>1,500(p<0.001)$ were more likely to have an AMT. Audits to measure policy adherence were more likely $(p<0.001)$ if an AMT was present.

Nurses $(89 \% \mathrm{n}=201)$ and pharmacists $(73 \% \mathrm{n}=165)$ were most likely to be involved in NMP of antimicrobials.

Conclusions: While most respondents reported an antimicrobial prescribing policy, less had an AMT. Despite recent government and regional initiatives, further improvements in antimicrobial stewardship are still required.

Disclosure of Interest: None Declared.

\section{HP-PC44}

\section{Importance of caregivers on medication discrepancies} during Hospital admission

J. J. Ramos Baez ${ }^{1, *}$, E. Campos Davila ${ }^{1}$, S. M. Marrero Penichet ${ }^{2}$, E. Marquez Fernandez ${ }^{1}$, M. P. Quesada Sanz ${ }^{1}$

${ }^{1}$ Pharmacy Department, Campo de Gibraltar Health Care Area, Cadiz, ${ }^{2}$ Pharmacy Department, H Universitario Dr Negrin, Las Palmas de Gran Canaria, Spain

Background and Objective: To analyze the differences in medication discrepancies between patients who live alone and those who live with caregivers when admitted on a surgical ward of a secondary level hospital. Setting and Method: A Prospective, Cohort Study conducted over a selection of patients. Inclusion criteria: Patients admitted at surgery ward, older than 50 and taking more than two drugs concomitantly. Data collection was performed in a month. Review of medical charts of selected patients and clinical interview with patient or caregiver in all cases.

Main outcome measures: Diagnosis (CIE-9 codification), comorbidities, planned admission to hospital (yes-no), medication discrepancies detected in drug treatment at hospital admission (justified, unjustified), recommendations (accepted, not accepted), gravity of unjustified discrepancies based on NCCMERP's scale, Patient living alone (yes-no), or living with caregiver (yes-no). The data analysis was performed using SPSS statistical package.

Results: 37 patients were included, mean age 72.4 years. $32.4 \%$ had more than 3 comorbidities. $27.02 \%$ of patients were living with caregiver versus $72.97 \%$ who lived alone. Discrepancies were found in $84.7 \%$ of patients $(67,74 \%$ in self-medicated patients), of which $44.5 \%$ were unjustified discrepancies. The most frequent discrepancies were omission on prescription and adjusting medication to the hospital pharmacotherapeutic guidelines. According to the study, unjustified discrepancies that were presented and accepted by the attending physician were considered medication errors, thus at least one error in reconciliation was found in $44.3 \%$ of patients (of which $77.7 \%$ was in patients living alone). $5 \%$ of errors were classified as potentially harmful if reconciliation shouldn't be done. In the group of patients who lived with care were detected $50 \%$ fewer errors than patients who lived alone.

Conclusions: The percentage of discrepancies on admission was overall elevated and showed that a reconciliation process is necessary, especially in self-medicated patients, because the probability of errors is higher. Caregivers provide better and more accurate information about medication during admission and it is reflected in a lower number of errors.

Disclosure of Interest: None Declared.

\section{HP-PC45}

Attitudes and experiences of off-label prescribing among paediatricians in a French university teaching hospital P. Joret $^{1,2, *}$, J. Bataille ${ }^{1,2}$, S. Prot-Labarthe ${ }^{1}$, F. Brion ${ }^{1,2,3}$, J.-F. Hartmann ${ }^{4}$, O. Bourdon ${ }^{1,2,3}$

${ }^{1}$ APHP, Pharmacie, Hôpital Robert-Debré, ${ }^{2}$ Pharmacie Clinique, Université Paris Descartes, Paris, ${ }^{3}$ Laboratoire Education et Pratiques de Santé, Université Paris XIII, Bobigny, ${ }^{4}$ Coordonnateur de la gestion des risques associés aux soins, CLIN/CVRiS, Responsable du management de la qualité de la prise en charge médicamenteuse, Hôpital Robert-Debré, Paris, France

Background and Objective: The lack of drugs specifically assessed for pediatric use results in a widespread off-label drug use. Existing data on off-label prescribing are based on prescription registers from hospitals but little is know about the perceptions and attitudes of paediatricians regarding off-label prescribing for children. Thus, the aim of this work is to identify experiences and attitudes towards paediatrics off-label prescribing in a university teaching paediatric hospital.

Setting and Method: A questionnaire of 24 items was sent by email to 409 paediatricians (SurveyMonkey ${ }^{\circledR}$ link) and answers were collected during a six weeks period. Experiences on off-label prescriptions were collected: frequency of prescribing for an indication not included in the license or approved for adults only, (2) outside the age range and/or weight range of the product license; sources of information; concern about safety and adverse events with off-label drug use; proportion of parents informed and order with "off-label" mention.

Main outcome measures: Obtain qualitative data on the attitudes and experiences of paediatricians.

Results: $80(20 \%)$ questionnaires were completed. Over $81 \%$ of physicians were familiar with the concept of off-label drugs prescribing. The most common reason given for off-label prescribing was for a younger age (74\%) and for another indication $(28 \%)$. The majority of respondents (79\%) used a colleague opinion and the most important sources of information used were the litterature $(72 \%)$, international guidelines (62\%), the French National Formulary $(56 \%)$ and national guidelines (46\%). Although $54 \%$ of paediatricians expressed concerns about safety about off-label prescription, only $29 \%$ had observed adverse event with off-label drug use. Two third of respondents informed the parents and one third expressed that off-label is widespread and can't be always explaining to family. 
Many respondents $(81 \%)$ did not write "off-label" mention on the prescription. However, $52 \%$ stated that they would be willing to undertake off-label prescription monitoring with a local observatory. Conclusions: French paediatricians are concerned by off-label prescribing. The survey underlines the need for clinical research for some drugs as a priority and the prospective collection of off-label prescribing.

Disclosure of Interest: None Declared.

\section{HP-PC46}

Optimization of antibiotic administration by continuous infusion in a home-care unit

A. Armand ${ }^{1, *}$, D. Peynaud-Vagneur ${ }^{2}$, G. Prévot ${ }^{3}$, A.-C. Feyel-Dobrokhotov ${ }^{1}$, S. Raymond ${ }^{1}$, A. Thomas ${ }^{1}$, L. Amico ${ }^{3}$, G. Rabatel ${ }^{1}$

${ }^{1}$ Pharmacie, Centre Hospitalier de Chambéry, Chambéry, ${ }^{2}$ Pharmacie, Hopitaux de Modane et Saint Jean de Maurienne, Saint-Jean-de-

Maurienne, ${ }^{3}$ Service Hospitalisation à domicile, Centre Hospitalier de Chambéry, Chambéry, France

Background and Objective: The home-care unit of Chambéry's hospital takes over patients that need intravenous antibiotic administration for severe infections, often after a hospitalization in the infectious disease department. The frequency of certain antibiotics administration is neither compatible with the unit organization nor economically viable (e.g. a 2-gram dose every four hours of cloxacillin sodium would need six nurse's visits per day). Thus a literature review was done to identify the best practices for antibiotic administration by continuous infusion when applicable.

Setting and Method: For the following antibiotics, a summary of the literature regarding stability data was done: amoxicillin sodium, amoxicillin sodium + clavulanic acid, ceftazidime, cloxacillin sodium, imipenem + cilastatin sodium, penicillin G sodium, piperacillin sodium + tazobactam, ticarcillin + clavulanic acid and vancomycin hydrochloride. A recap of patients that had benefited from a continuous infusion administration in our home-care unit since April 2012 was performed.

Main outcome measures: In order to fit the feasibility and methods of administration for each antibiotic, we studied several parameters: dose, diluent, concentration, drug delivery system (elastomeric reservoir pump with a capacity of $240 \mathrm{ml}$ over $24 \mathrm{~h}$ or infusion bag controlled by a pump), container (PVC, PVC-free, elastomer) and infusion time.

Results: Only amoxicillin sodium + clavulanic acid is too unstable to be administrated by continuous infusion. Amoxicillin sodium, imipenem + cilastatin sodium and penicillin $G$ sodium are the least stable antibiotics that lead to a compulsory change of the drug delivery system every $12 \mathrm{~h}$. For the others, the elastomeric reservoir pump or infusion bag can be used over $24 \mathrm{~h}$; the choice between these two systems depends on the administrated volume. Since April 2012, 63 patients have been treated with this method of administration for the following infections: bone, lung, urinary infections, endocarditis and septicemias. The main used antibiotics are: ceftazidime (23\%), cloxacillin sodium $(22 \%)$, piperacillin sodium + tazobactam $(20 \%)$, vancomycin hydrochloride $(12 \%)$, imipenem + cilastatin sodium (11\%) and amoxicillin sodium (8\%).

Conclusions: Several antibiotics are stable enough for a continuous infusion administration in a home-care unit. Administration protocols validated by the pharmacy and home-care units have been done according to the antibiotic dosage and the pathophysiology state of the patient (renal insufficiency, diabetes...). These protocols have been set up in our prescription software in order to facilitate the antibiotic prescription and secure their administration.

Disclosure of Interest: None Declared.

\section{HP-PC47}

Regional assessment of the crushing medications audit

F. Sohouenou ${ }^{1}$, C. Borel ${ }^{1, *}$, G. Bourdenet ${ }^{2}$, O. Nicol $^{3}$, M. Lefevre-Caussin ${ }^{1}$, J. Doucet ${ }^{1}$, E. Remy ${ }^{1}$

${ }^{1}$ OMEDIT Upper Normandy, ${ }^{2}$ Geriatric internal medicine, ${ }^{3}$ Department of Pharmacy, CHU, Rouen, France

Background and Objective: Crushing drugs is frequently carried out in health facilities (HF).

Following an initial study in a university hospital and the publishing of regional and national guidelines (HAS), the Observatory of Drugs, Medical Devices and Therapeutic Innovation conducted a regional synthesis of practices regarding crushing medications from prescription to administration.

Setting and Method: A common file was sent to all of the regional HF. The audit had to be carried out in two departments on 30 medical records of patients concerned with crushing tablets or opening capsules. The audit had to be done on a single period of administration (morning, afternoon or evening) for up to 1 month.

Main outcome measures: The outcome was measured with the 2012 good practice contract report.

Results: In total, 376 patients ( $61 \pm 11$ years) in $27 \mathrm{HF}$ received 1,154 crushed or opened drugs ( $3 \pm 1$ per patient), most often in the morning (43\%). The main reasons of crushing were swallowing disorders and psycho-behavioral disorders. The crushing of a pharmaceutical form was contraindicated in $26 \% \pm 9 \%$ of cases and $28 \% \pm 10 \%$ of the crushed drugs had a therapeutic alternative. Drugs of the same patient were crushed together ( $43 \%$ ), with a material used for several patients (54\%, mortar or grinder-crusher) but mostly cleaned between patients (55\%). The administration was performed in $65 \%$ of cases by nurses and $14 \%$ by nursing auxiliaries. A vehicle other than water \pm gel was used for administration in $44 \%$ of cases. The cleaning was mainly done with water \pm detergent $(41 \%)$. The drugs were administered immediately after preparation in $84 \%$ of cases.

Conclusions: Crushing drugs exposes to iatrogenic risks and occupational hazards. Our results show an improvement of practices, but also strong disparities inter and intra HF regarding procedures of preparation and/or administration of the crushed medicines. Regional recommendations and a regional list of crushable drugs regularly updated will therefore be published again.

Disclosure of Interest: None Declared.

\section{HP-PC48}

Prescription of rituximab in Rheumatology: audit of practices

A. L. Bourgeois ${ }^{1}$, A. Thole ${ }^{1}$, T. Salamon ${ }^{1}$, P. Calvet ${ }^{1, *}$, A. Cantagrel ${ }^{2}$, E. Civade ${ }^{1}$, M. C. Morin ${ }^{1}$

${ }^{1} 31$, CHU Purpan, Equipe Pharmaceutique Pôle Institut Loco Moteur, ${ }^{2} 31$, CHU Purpan, Service de Rhumatologie, Toulouse, France

Background and Objective: Rituximab, anti-CD20 antibody, is a biotherapy indicated in the treatment of severely active rheumatoid arthritis (RA) of the adult. The guidelines recommend use, in association with methotrexate after failure or therapeutic escape with another disease-modifying therapy including at least one anti-TNF alpha. The objective of our study is to assess prescribing practices of rituximab.

Setting and Method: A retrospective study was conducted in three Rheumatology units from May 2012 to April 2013, using data from computerized patient records.

Main outcome measures: The main criteria evaluated are: indication (RA or other), whether associated with methotrexate or not, and 
whether previous disease-modifying drugs had been tried. We also identified the administered dose, the number of infusions per cycle, the treatment during intercycle and causes of reprocessing.

Results: 84 patients were treated, amongst which 77 for RA and 7 for other pathologies, including 14 men for 70 women. In the RA group, $45.2 \%$ of patients were treated with association rituximab/methotrexate while $54.8 \%$ were treated with rituximab alone, but this strategy was justified in more than half of the cases: intolerance, ineffectiveness, adverse effects, contraindications or therapeutic failure. In $88 \%$ of cases, prior use of anti-TNF alpha was found. $98.3 \%$ of the patients received $1 \mathrm{G}$ of rituximab per infusion. Two infusions per cycle were done for 68 patients $(88.3 \%)$ and one infusion for 9 patients $(11.7 \%)$. The length of intercycle was of an average 15.8 months, with 11 patients $(26.8 \%)$ reprocess systematically and 30 patients $(73.2 \%)$ for a disease flare. The prescriptions for non-RA pathologies concerned only $8.3 \%$ of patients and were mainly seen in the Sjögren syndrome and mixed connective tissue diseases. Concerning the Sjögren syndrome, this indication is off label and has not been approved by our health authorities. However, numerous studies indicate that an improvement of fatigue [1], synovial infringement, salivary flow [2] and cutaneous and neurological manifestations are observed following infusions of rituximab in this disease.

[1] Daas S, Bowman SJ, Vital EM, et al./Reduction of fatigue in Sjögren syndrome with rituximab: results of a randomized, doubleblind, placebo-controlled pilot study. Ann Rheum Dis2008;67/1541-4

[2] Meijer JM, Meiners PM, Vissink A, et al. Effectiveness of rituximab treatment in primary Sjögren's syndrome: a randomized, double-blind, placebo controlled trial. Arthritis Rheum 2010;62:960-8 Conclusions: Prescribing practices within our University Hospital are in accordance with the recommendations. Rituximab is increasingly prescribed in autoimmune diseases, with an increased interest. The place of the pharmacist is thus essential in order to regulate off-label prescriptions, first of all to avoid misuse but also from a financial point of view. Clinicians will be informed of these results to in order to increase their awareness.

Disclosure of Interest: None Declared.

\section{HP-PC49}

Intravenous Immunoglobulins: a treatment for Narcolepsy with Cataplexy?

\section{J. Corny ${ }^{1, *}$, A. Papon ${ }^{1}$, M. Lecendreux ${ }^{2}$, F. Brion ${ }^{1}$, O. Bourdon ${ }^{1}$}

${ }^{1}$ Pharmacy, ${ }^{2}$ Pediatric Sleep Disorders Center, Hopital Robert Debré, Paris, France

Background and Objective: Narcolepsy is a neurological disorder caused by the brain's inability to regulate sleep-wake cycles normally. Its prevalence dramatically increased after AS03 adjuvanted pandemic $\mathrm{A}(\mathrm{H} 1 \mathrm{~N} 1)$ vaccination campaign during winter 2009-2010, suggesting an immune origin. In this context, intravenous immunoglobulins (IVIg) have been used. The aim of this study is to evaluate the efficiency and the tolerability of this treatment in pediatric patients.

Setting and Method: A literature review was performed in December 2012. Then, we conducted a retrospective study between January 2010 and December 2012 to evaluate the efficiency of IVIg in this disease. We collected data in medical files after identifying narcoleptic patients who received IVIg. Follow-up period started one month before the first IVIg administration and ended one month after the last one.

Main outcome measures: Efficiency criteria used were the evolution of pediatric narcolepsy scale scores (Pediatric Daytime Sleepiness
Scale-PDSS, and cataplexy scale-CGI-C) and the sleep latency increase (Multiple Sleep Latency Test-MSLT). Statistic test used was Student's t-test with paired samples.

Results: Only one double-blind placebo-controlled study $(\mathrm{n}=1)$ was found and didn't show a significant difference between placebo and IVIg. No clinical trial was reported in clinicaltrial.gov.

In our hospital, 23 pediatric narcoleptic patients received IVIg ( $1 \mathrm{gram} / \mathrm{kg}$ ) on a 4-week interval basis between January 2010 and December 2012 (mean age: 12.2 years old). $69.6 \%$ received the AS03 adjuvanted pandemic $\mathrm{A}(\mathrm{H} 1 \mathrm{~N} 1)$ vaccine. Some of these patients also received concomitant psychostimulant-based treatment (methylphenidate or modafinil).

For patients without change in psychostimulant-based treatment during the follow-up period (39.4\%), the study showed a statistically significant decrease in cataplexy frequency $(\mathrm{n}=16, p=0.09)$ and in excessive daytime sleepiness $(\mathrm{n}=8, p=0.017)$, and a statistically significant increase in sleep latency $(\mathrm{n}=5, p=0.02)$.

Conclusions: It seems that IVIg can reduce excessive daytime sleepiness and cataplexy frequency. These findings are encouraging but need further analysis. This is why a multicenter case-control study and a long-term follow-up study are currently being conducted. Disclosure of Interest: None Declared.

\section{HP-PC50}

\section{Oral versus parenteral route of application: The patient's preference}

\section{Metaxas ${ }^{1, *}$, A. Schmutz ${ }^{1}$, P. Walter ${ }^{1}$, K. E. Hersberger ${ }^{1}$}

${ }^{1}$ Dept. Pharmaceutical Sciences, University of Basel, Basel, Switzerland

Background and Objective: Many drugs can be applied either by oral or parenteral therapy. The preferred route of administration is often predetermined by indication, affordable dose and therapeutic setting. Substitution of vitamin B12 deficient outpatients is one example where the equivalent efficacy of oral and intramuscular application offers both opportunities. Wherever alternative routes of administration exist, the patients' preference should represent a considerable criterion to choose between the treatment options. Our objective was to review current knowledge on patient's preference. Setting and Method: Trials were identified by Pubmed and EMBASE search (1980-2013) using the terms "patient/s' preference", "oral" AND "inject*". Original research articles which were accessible online and included intravenous, intramuscular and subcutaneous treatments to compare with the oral therapy option were compared. References of all identified trials were also checked for other studies. The research articles were classified by indication and treatment, number of patients, assessment method, and patient preference.

Main outcome measures: number of studies with preference for oral treatment; qualitative: assessment methods, factors with a presumed influence on preference.

Results: We retrieved 13 articles dealing with the acceptance of oral vs. parenteral cancer treatment (5), antibiotic therapies (3), substitution of vitamin deficiency (2) or other indications (3). Eleven articles reported a preference for the oral administration. Associated factors with preference for the two routes varied between the studies. Most articles reported convenience as an important factor to influence preference, either in favourite of the oral or the parenteral therapy. A variety of different questionnaires and study designs were used to assess patient preference. Two studies applied validated methods and the remaining 10 studies specifically designed questions. Out of them, 
7 questionnaires used 5-10 point Likert scales and/or multiple answer options, and two surveys used open questions. One survey was conducted as a scenario based interview.

Conclusions: The small number of available studies revealed a tendency towards preference for oral treatment, which was mostly influenced by the demand for convenience and the patients' disease. These findings are in line with our own unpublished observations in a pilot study on vitamin B12 deficient patients. Interestingly, we found patients to prefer parenteral therapy due to an unjustified belief in the superiority of clinical effects; same was reported by three other studies. Hence, in any situation of medical uncertainty it is important to consider patients' preference, evaluate the risk of non-adherence and end up with shared decision making. Additional research with validated methods is essential to gain insight in the patients' perspective, especially in therapy options with comparable efficacy and safety.

Disclosure of Interest: None Declared.

\section{HP-PC51}

Effectiveness of the paclitaxel releasing balloon Pantera Lux ${ }^{\circledR}$ in diabetic patient with in-stent restenosis? A case report

G. Foy ${ }^{1, *}$, A. Jacob ${ }^{1}$, G. Sideris ${ }^{2}$, A.-C. Steinmetz ${ }^{1}$, H. Barreteau ${ }^{1}$

${ }^{1}$ Pharmacy, ${ }^{2}$ Cardiology, Lariboisiere Hospital, AP-HP, Paris, France

Background and Objective: Diabetes is a risk factor of coronary in-stent restenosis (ISR) after treatment with either bare metal stent (BMS) or drug-eluting stent (DES). Depending on their localization and intrinsic characteristics, treatment of ISR could be very challenging. These last years, new drug-eluting balloons (DEB) have emerged as a valuable medical device and innovative clinical option to treat ISR. We report here the efficacy of the paclitaxelreleasing balloon Pantera $\operatorname{Lux}^{\circledR}$ in a diabetic patient presenting an ISR.

Setting and Method: Cardiology unit, Lariboisière Hospital, Case report.

Main outcome measures: In 2002, a 55 years-old man presented an acute coronary syndrome treated by angioplasty and a BMS implantation. In 2010, multiple de novo coronary stenosis were evidenced. Since the patient was now suffering from type 2 diabetes, six DES were implanted. In March 2013, following a clinical deterioration of his chronic angina, the patient was hospitalized. Angiography revealed a significant ISR with a $95 \%$ coronary diameter reduction. The anatomy of the lesion and previous multiple DES implantations didn't allow to deploy a new stent. Therefore clinician decided to practice an innovative revascularization therapy: expansion with DEB using the Pantera Lux ${ }^{\circledR}$ medical device.

Results: The procedure of ISR expansion with Pantera Lux ${ }^{\circledR}$ was successful. Clinician reported a simple, rapid and easy application of the device. Angiographic data evidenced an immediate significant diameter stenosis reduction of $60 \%$. No major adverse cardiac event occurred. The control coronary angiography at 3 months showed a lasting result with a stable residual stenosis of $40 \%$. Moreover the patient reported substantial clinical benefits regarding his chronic angina.

Conclusions: This case showed efficiency of the paclitaxel releasing balloon Pantera Lux ${ }^{\circledR}$ in revascularization of an ISR in a diabetic patient. A recent meta-analysis also established that DEB significantly reduced the need for revascularization and major cardiac adverse events as compared to angioplasty or DES at 1- to 5-year follow-up (Indermuehle et al., 2013). Therefore DEB are likely to be a promising tool and innovative therapy in the treatment of ISR.

Disclosure of Interest: None Declared.
HP-PC52

Patterns of use of trabectedin in a French centre

L. Sylvestre ${ }^{1}$, A. El Aatmani ${ }^{1}$, J.-E. Kurtz ${ }^{2}$, I. Asmane-De La Porte ${ }^{2}$, P. Barthelemy ${ }^{2}$, D. Leveque ${ }^{1, *}$, B. Gourieux ${ }^{1}$

${ }^{1}$ Pharmacy-Sterilization, ${ }^{2}$ Oncology, University Hospital, Strasbourg, France

Background and Objective: Trabectedin is a complex alkylating agent approved for treatment of advanced soft tissue sarcoma after failure of anthracyclines and ifosfamide. Data relative to its use in clinical practice are scarce. The goal of our study was to examine the pattern of trabectedin use in a French centre.

Setting and Method: We identified all advanced soft tissue sarcoma patients given trabectedin between 2007 and 2012 through the pharmacy database. All medical files were retrospectively investigated to describe the patterns of trabectedin prescription and administration.

Main outcome measures: Recorded data included patients' demographics and medical history, patterns of trabectedin prescription. Safety data were collected from patients' charts as well as outcome. Results: We identified 17 patients (male-to-female ratio: 0.7) with a median age of 56 years (range, 25-75 years). Patients had leiomyosarcoma (n:6), liposarcoma (n:3), synovialosarcoma (n:3), fibrosarcoma (n:2) or other subtypes (n:3). All patients had metastatic disease and the median number of metastatic organs was 3 (range, 1-7). They had undergone surgery and 6 had received radiotherapy. Sixteen had received prior chemotherapy with either anthracycline or ifosfamide or the two treatments whereas the other one had received gemcitabine and docetaxel for uterine leiomyosarcoma. Trabectedine (1.5 or $1.2 \mathrm{mg} / \mathrm{m}^{2}$ ) was given intravenously over $24 \mathrm{~h}$, every 3 weeks as third-line therapy on average (range 2-5),second line therapy in 4 patients $(24 \%)$, third-line therapy in $5(29 \%)$, fourth-line therapy in 6 (35\%)and fifth-line therapy in $2(12 \%)$. All patients received intravenous dexamethasone before trabectedine infusion and granulocyte colony-stimulating factor support after. The median number of cycles was 3.9 (range, 1 to 12 ) and three patients received $\geq 6$ cycles. Discontinuations occured to disease progression $(n=10)$ and adverse effects $(n=3)$. During the course of the treatment, $6 / 17$ patients required dose adjustments (dose reduction and/or cycle delays) due to toxicities. Fifteen patients presented at least one adverse event and two required hospitalization. The main adverse events were hematologic toxicity (n:9) including febrile neutropenia (n:5) and biochemical toxicity (transaminases and bilirubin elevations, n: 3 ). Seven patients continuated treatment after trabectedin.

Conclusions: Trabectedin was used in highly pretreated patients with sarcomas among them half had leiomyosarcoma or liposarcoma. Toxicity of trabectedin was significant. The duration of treatment was short, in line with compassionate use programs.

Disclosure of Interest: None Declared.

\section{HP-PC54}

A continuous improvement program: toward the securisation of pre-operative order for planned surgery

A.-S. Monfort ${ }^{1}$, L. Gutermann ${ }^{1}$, M.-C. Chaumais ${ }^{1}$, D. Christensen ${ }^{2}$, S. Foucher ${ }^{1, *}$, A. Rieutord ${ }^{1}$, S. Roy ${ }^{1}$, R. Haddad ${ }^{1}$ ${ }^{1}$ Pharmacy, ${ }^{2}$ Anesthesia, Hôpital Antoine Béclère, AP-HP, Clamart, France

Background and Objective: Since January 2011, pharmacy department at Antoine Béclère hospital conducted Medication History ( $\mathrm{MH})$ both in visceral and orthopaedic surgery wards. Among 1,800 annual 
$\mathrm{MH}, 40 \%$ involved patient with planned surgery. During pre-anesthesia consultation, the anesthesic form (AF) is completed and currently used as reference for the preoperative order. In 2012, a continuous improvement program was implemented to increase the concordance between $\mathrm{AF}$ and $\mathrm{MH}$. A medication plan has been integrated in the $\mathrm{AF}$ and a letter was sent to patients prior to anesthesia consultation asking them to bring their prescription. Both measures aimed to improve the completeness of personal treatments carried over AF.

The aim of our study was to evaluate the impact of these changes regarding the concordance with the $\mathrm{MH}$.

Setting and Method: Two four-weeks prospective studies (Sept. 2012 and May 2013) have been conducted to assess the concordance between $\mathrm{AF}$ and $\mathrm{MH}$ before and after modifications. All patients with planned-surgery and at least one medication on the $\mathrm{MH}$ were included. Main outcome measures: Discrepancies between $\mathrm{MH}$ and $\mathrm{AF}$ were split into two groups: medication (additional or missing drug, incorrect or omitted dosage) and administration plan (incorrect or omitted). Results: Thirty patients were included in the first study. MH mentioned 110 medications, 104 on the AF. Forty patients were included in the second study. MH mentioned 150 medications, 158 on the AF.

The overall concordance (the right medication with the right dosage and administration plan) between $\mathrm{MH}$ and $\mathrm{AF}$ was $10 \%$ in both studies. The concordance in terms of medication was significantly lower in the second study: $76.7 \%$ vs. $47.5 \%(p \leq 0.01)$. This is explained by the number of missing or additional drugs which was significantly higher in the second study: $9.1 \%$ vs. $30.7 \%(p<0.01)$.

The number of incorrect dosages was not significantly different in both studies ( 12.1 vs. $6.5 \%)$ while the number of missing dosages was significantly lower in the second study: 44.2 vs. $28.7 \%$ $(p<0.01)$

Finally, no significant difference was observed on the number of missing (11.5\% vs. $6.7 \%)$ or false (12\% vs. $19.3 \%)$ administration plans.

Conclusions: The medication plan integration in the AF has reduced the number of omitted dosages. Despite of our expectations, the presence of the patient's order during pre-anesthesia consultation did not improve the completeness of personal treatments on the AF.

These high non-conformity rates can lead to medication errors and highlight the inadequacy of the current chart with daily practice. They also reveal that the current improvement measures are insufficient.

A multidisciplinary team is implementing news improvement measures: new medication plan in AF, a training of the healthcare professional before implementation and integration of pharmacy student who conducted MH upstream during pre-anesthesia consultation.

A reassessment will be scheduled after these changes.

Disclosure of Interest: None Declared.

\section{HP-PC55}

Evaluation of the implementation of a classification system for pharmaceutical interventions

\author{
K. A. Maes ${ }^{1, *}$, A. Gaufroid ${ }^{1}$, M. Messerli ${ }^{1}$, K. E. Hersberger ${ }^{1}$, \\ M. L. Lampert ${ }^{1,2}$ \\ ${ }^{1}$ Pharmacy, Pharmaceutical Care Research Group, University \\ of Basel, Basel, ${ }^{2}$ Clinical pharmacy, Kantonsspital Baselland, \\ Bruderholz, Switzerland
}

Background and Objective: The Swiss Society of Public Health Administration and Hospital pharmacists (GSASA) introduced in 2011 a new GSASA classification system for pharmaceutical interventions in Swiss hospitals. The instrument, developed and validated in a previous research, comprises five main categories (detected problem, type of problem, cause of intervention, intervention, and outcome of intervention). Our objectives were to evaluate the implementation of this classification system in daily practice, and to analyse the pooled data retrieved from Swiss hospitals.

Setting and Method: Chief pharmacists $(n=47)$ were asked by online questionnaire about the use and satisfaction with their classification system. We asked them to provide voluntarily their data containing all interventions classified with the GSASA system during daily work. We evaluated the user satisfaction about comprehensiveness, feasibility, and acceptability by a 5-point Likert scale ( $1=$ strongly disagree, $5=$ strongly agree $)$. We standardised the provided data and performed descriptive analyses.

Main outcome measures: Extent of use of the GSASA classification system, user satisfaction, comprehensiveness of the classification.

Results: The questionnaire was completed by 45 chief pharmacists (96\%), therefrom 34 hospitals offer regularly clinical pharmacy services $(76 \%)$ and 7 planned it $(16 \%)$. Twelve hospitals $(27 \%)$ used the GSASA classification system, 25 used another system (55\%, most of them [20] used an individual in-house system), and 7 used no structured classification (16\%). From 11 hospitals, we received 9,543 pharmaceutical interventions classified with the GSASA system during 121.5 months (sum of months per hospital). Of all interventions, $8.8 \%$ were not fully classifiable $(\mathrm{n}=840)$. This is reflected by the user opinion: 6 of 12 users were not satisfied with the comprehensiveness of the system (mean user agreement $2.9 \pm 1.1$ ). In general, users were satisfied $(3.8 \pm 0.9)$ with the GSASA system, especially with the adequate time expenditure $(4.1 \pm 1.0)$. Ten users reported to need $<2 \mathrm{~min}(83.3 \%)$ and $2(16.7 \%)$ up to $4 \mathrm{~min}$ to classify one intervention.

Conclusions: The GSASA classification system is systematically used and widely accepted in Switzerland. Users are still satisfied 18 months after its implementation. This practical instrument is suitable to almost completely classify pharmaceutical interventions in daily practice with an adequate time effort. Further refinements will increase the user satisfaction.

Disclosure of Interest: None Declared.

\section{HP-PC56}

Pharmacist impact on inpatient medication discrepancy: a 1 year evaluation in a french internal medicine unit

\section{R. Stehle ${ }^{1}$, Y. Audurier ${ }^{1}$, M. Villiet ${ }^{2, *}$, P. Fesler ${ }^{1}$, J. Ribstein ${ }^{1}$,} C. Breuker ${ }^{2}$, S. Hansel-Esteller ${ }^{2}$

${ }^{1}$ Internal Medicine, ${ }^{2}$ Pharmacy Lapeyronie, CHRU Montpellier, Montpellier, France

Background and Objective: Medication reconciliation is a formal process for creating the most complete and accurate list possible of a patient's current medications and comparing the list to those in the patient record or medication orders. The objective of this study was to evaluate the impact of medication reconciliation made by clinician pharmacist.

Setting and Method: A 1 year prospective study of in-patients in an internal medicine department was conducted. Patients included were hospitalized $>24 \mathrm{~h}$ and were treated by $>4$ drugs. The pharmacy resident used different data sources: patient/family interviews, general practitioner prescription and community pharmacy.

Main outcome measures: The following data were collected: patient name, age, gender, reason for hospitalization, comorbidities and drugs history. A comparison between the first drug prescription and the prehopitalisation prescription was performed. Discrepancies were categorized with the clinician as Non Intentional Discrepancy (NID) or Intentional Discrepancy (ID). The pharmaceutical interventions were registered: type of discrepancies, related drug (using the Anatomical Therapeutic Chemical classification system) and physician correction. 
Results: Medication of $277 / 390$ admitted patients were conciliated. Mean age of patients was $77.7 \pm 12.4$ years and the average number of drugs taken was $8.54 \pm 3.15$. A total of 2,331 prescription lines were analyzed, and 1,409 discrepancies were detected: 118 NID and 1,291 ID. At least one NID occurred in 67 patients $(24.2 \%)$. The most frequent discrepancies were observed with psycholeptics $(10.2 \%)$, lipids modifying agents $(7.6 \%)$, thyroid therapy $(6.8 \%)$, drug used in diabetes $(6.8 \%)$, antithrombotic agents $(6.8 \%)$, agents acting on the renin-angiotensin system $(6.8 \%)$, drugs for acid related desorders $(5.9 \%)$ and psychoanaleptics $(5.9 \%)$. Main reasons for NID were omission of a drug $(44.0 \%)$ increase $(20.3 \%)$ or reduction $(18.7 \%)$ of the drug dose and initiation $(8.5 \%)$. Acceptance of pharmaceutical recommendation by physicians was $88.9 \%$.

Conclusions: We demonstrate the significant number of NID, reaching almost a quarter of included patient. The drug omissions are the most frequent NID. Drug reconciliation avoids NID and adverse drug event across transitions in care and improves the safety of using medications. This study demonstrates the role of the clinician pharmacist, as part of the multidisciplinary team, in detection and correction of NID.

Disclosure of Interest: None Declared.

\section{HP-PC57}

Investigation into the usage of hypodermoclysis in the geriatric units

\section{A. Bigot ${ }^{1, *}$, L. Boccanfuso ${ }^{1}$, L. Rouch ${ }^{1}$, C. Lebaudy ${ }^{1}$, S. Gérard ${ }^{2}$, P. Cestac ${ }^{1}$ \\ ${ }^{1}$ Pharmacie Gériatrie, ${ }^{2}$ Médecine Gériatrique, Chu Toulouse Purpan Casselardit, Toulouse, France}

Background and Objective: The practice of hypodermoclysis is used for the administration of fluids into the subcutaneous tissue. This is the alternative delivery method when the oral canal is unusable and intravenous infusion is not possible, which is often the case with elderly patients. The goal of this study was to identify the factors that contribute to complications when practicing hypodermoclysis in geriatric units.

Setting and Method: We conducted a prospective observational study, over a period of 6 weeks in the Geriatric Department. Nurses and pharmacy students collected information specific to the practice of hypodermoclysis.

Main outcome measures: To conduct this study we are more particularly interested on skin antisepsis, composition of infusion, puncture site, duration of infusion and the occurrence of complications.

Results: For this study, 115 patients were given a hypodermoclysis with an average volume of 1 litre and an average infusion duration of $12 \mathrm{~h}$. Drugs were added to the infusion in $14.7 \%$ of the cases (diazepam, nefopam and scopolamine). In $9 \%$ of the cases, the catheter used was not the catheter recommended for subcutaneous administration. Aseptic conditions were incorrect in $45 \%$ of the cases.

12 complications were observed (this represents $10 \%$ of the patients). Inflammation occurred in the majority of the cases sometimes associated with bleeding ( 1 case), abscess ( 2 cases), or flow of pus ( 2 cases). Complications were observed in $18.9 \%$ of cases when the catheter was located on the thigh, $7.1 \%$ when on the abdomen and $0 \%$ when located on other sites (arm, back, sub-clavicular zone). In two cases, diazepam and scopolamine were added to the rehydration solution.

Conclusions: Diazepam is irritating for the subcutaneous tissue and should be avoided when using continuous infusion. The complication rate appears to be similar to those observed in the literature i.e. $12 \%$ (1).

In order to reduce the number of complications resulting from malpractice, we will examine professional practices and perform training when necessary.
1.Arinzon Z, J Feldman, Fidelman Z, Gepstein R, Berner YN. Hypodermoclysis effective mode of treatment of dehydration in longterm care patients. Arc Gerontol Geriatr 2004 Mar-Apr; 38 (2) :167-73 Disclosure of Interest: None Declared.

\section{HP-PC58}

Pharmacists and clinicians collaborate: inter-disciplinary team for the clinical risk management in Italy

\section{E. Galfrascoli ${ }^{1 \text {,* }}$ and Working Group FARMAONCO}

${ }^{1}$ Hospital Pharmacy, A.O. Fatebenefratelli e Oftalmico, Milan, Italy

Background and Objective: The clinical risk management is aimed at the prevention of avoidable mistakes, in order to limit their possible harmful effects, to ensure patients safety.

The development of effective interventions is related to the implementation of organizational measures to promote the analysis of adverse events.

Setting and Method: In Lombardy since 2006, the 'Lombardy Oncology Network-Rete Oncologica Lombarda' (ROL), enables the exchange of clinical information among health care professionals involved in the prevention and treatment of cancer, ensuring a greater efficiency.

Since 2009, a pharmacovigilance project was also started in Lombardy: the FARMAONCO project, focused on reporting ADRs to drugs used in cancer treatment. It involves 13 hospitals, with the aim to increase the awareness of healthcare professionals of ADR in oncology.

A collaboration was started between ROL and FARMAONCO: a Working Group, made up of oncologists, pharmacists and nurses developed a work package aimed at improving the clinical risk management. Several useful documents and tools have been developed and are now shared on the ROL network.

Results: On the ROL website (http://www.progettorol.it/) a specific section for Pharmacovigilance and Clinical Risk Management was identified, for the release of the documents.

In particular, several macro-areas have been identified, concerning various important aspects and topics for cancer healthecare professionals: in these macro-areas the components of the Working Group cooperated accordingly with the different skills.

From June 2012, the Working Group has produced:

- dilution protocols for injectable drugs (66 cytotoxic drugs and 9 monoclonal antibodies)

- patient information leaflets for oral cancer drugs (13 drugs)

- 1 extravasation management protocol

- 1 document on pharmacogenetic tests, useful for the choice of cancer therapy

- 1 document on the risk-sharing agreements provided by AIFA (the Italian Medicines Agency) for drugs undergoing intensive monitoring.

A specific section for the clinical risk management legislation was also created, and a summary of documents on the pharmacovigilance signals released by AIFA was produced (12 antiblastic drugs and 2 supportive therapies). For 2013, we intend to integrate what has been already produced with additional tools for the clinical risk management (drug-drug interactions, food-drugs interactions, Failure Modes Effects and Criticality Analysis (FMECA), etc.).

Conclusions: Health system is a complex system in which all elements must be integrated to meet patients care needs: only with the collaboration between clinicians and pharmacists the safety of care and protection of patients can be reached.

Disclosure of Interest: None Declared. 


\section{HP-PC59}

Evaluation of the chemotherapy protocols and premedications associated in digestive oncology clinical departments in a french university hospital

\section{F. Le Quellec ${ }^{1, *}$, V. Servant ${ }^{1}$, A. Berroneau ${ }^{1}$, F. Xuereb ${ }^{1}$, D. Breilh ${ }^{1}$}

${ }^{1}$ Pharmacie, Chu Bordeaux, Bordeaux, France

Background and Objective: Haut-Lévêque hospital of the University hospital of Bordeaux account 3 wards of hepatogastroenterology. Because of the former creation of chemotherapies protocols on the CHIMIO $^{\mathrm{TM}}$ software, it seemed necessary to estimate their adequacies to the current international recommendations.

Setting and Method: It is a prospective study realized on one month (January 14th-February 14th, 2013) about the administration plans edited after prescriptions of chemotherapies with the CHIMIO ${ }^{\mathrm{TM}}$ software. This study is follows three axes:

1. Collect and analyze the administration plans in patient records,

2. Analyze possibly corrections made on the administration plans by caregivers,

3. Observe the administration of antineoplastic treatments in wards to confront it with the current recommendations.

Results: (1) Through the analysis of the administration plans, we observed:

- 145 administration plans collected correspond to 17 different chemotherapy protocols.

- 34 administration plans ( $24 \%$ ) do not correspond with the current recommendations even if the last modifications of these protocols date year 2012 .

- The main part of the non conformity corresponds to an unsuitable premedication: essentially a not appropriate antiemetic prophylaxis (absence of setrons in protocols FOLFIRINOX or FOLFOX) or an insufficient or unsuitable hyperhydration (all the protocols with cisplatin).

- Inconsistent administration time in the protocols were also found as well as the presence of physicochemical interactions (5 FU administered simultaneously to the calcium folinate for example in the protocol Dacarbazine-LV5FU2).

(2) Of the analysis of the corrections made by the caregivers: only 8 administrations plans were corrected. All the corrections concerned only one protocol: the insufficient hyperhydration in the protocol GEMCIS.

(3) Of the observation of the antineoplastic protocols administration: some practices do not correspond to protocols. In particular, the administration of fixed dosage not adapted in body area of calcium folinate and the administration of excessive dosage of magnesium sulfate. These practices were quickly corrected.

Finally, it was highlighted a not optimal administration of the treatment and especially premedication in 24 cases studied/145 that is $16.5 \%$ of the administrations

Conclusions: Further to this study, 9 protocols were modified, such as the associations of 5-fluorouracil with platinum salts, irinotecan and docetaxel. Administration plans are now applicable without modification.

This study demonstrates that a revaluation at least annual of the protocols is essential to guarantee the medical quality care of the patient.

The update of the premedication, sometimes left in the background by the prescribers, should be the subject of a special attention to optimize the tolerance of treatments and to respect better the doseintensity of protocols.

Thus, the role of the clinical pharmacist in oncology seems essential to optimize the efficiency of the treatments.

Disclosure of Interest: None Declared.

\section{HP-PC60}

\section{Botulinum toxin: in which indications?}

A. L. Bourgeois ${ }^{1}$, A. Thole ${ }^{1}$, T. Salamon ${ }^{1}$, P. Calvet ${ }^{1, *}$, B. Fraysse ${ }^{2}$, E. Civade ${ }^{1}$, M. C. Morin ${ }^{1}$

131, CHU Purpan, Equipe Pharmaceutique Pôle Institut Loco Moteur, 231, CHU Purpan, Service d'ORL, Toulouse, France

Background and Objective: Produced by the bacteria Clostridium botulinum, botulinum toxin is hazardous food contaminant with neurotoxic properties. However, it is used in the treatment of various diseases: hemifacial spasm, blepharospasm... The objective of our study is to assess prescribing practices of botulinum toxin.

Setting and Method: From computerized patient records, a retrospective study was conducted within 2 units of our University Hospital, Maxillofacial Surgery and Otorhinolaryngology, over a period of 6 months, from January, 2013 to June, 2013.

Main outcome measures: The primary endpoint is the percentage of prescriptions of botulinum toxin in accordance with Marketing Authorization (MA). Any off-label prescription is justified by bibliographic research.

Results: We extracted 68 prescriptions of botulinum toxin over the 6 month period. 6 could not be exploited. Among 62 analyzable prescriptions, $53 \%$ complied with MA, $47 \%$ were off-label and were mainly seen in maxillofacial surgery. The main indications we observed and that complied with MA were hemifacial spasm (25/33), blepharospasm (2/33) and the local symptomatic treatment of lower/ superior limbs (2/33). 4 patients were treated for aesthetic purposes (lines between the eyebrows) but in compliance with the MA of Vistabel ${ }^{\circledR}$. The three main off-label indications we saw were: bruxism (9/29) [1], hypersalivation (6/29) [2] and temporomandibular disorders $(5 / 29)[3]$.

[1] Santamato A, Panza F, Di Venere D, Solfrizzi V, Frisardi V, Ranieri M, Fiore P. Effectiveness of botulinum toxin type A treatment of neck pain related to nocturnal bruxism: a case report. J. Chiropr Med 2010;9(3):132-7

[2] Kossmehl P, Freundeberg B, Wissel J. Experience with a new botulinum toxin type A formulation in the treatment of sialorrhoea.

[3] Schwartz M, Freund B. Clin J Pain. 2002 Nov-Dec;18(6 Suppl):S198-203

Conclusions: Approximately $50 \%$ of prescriptions of botulinum toxin are off-label. However, these indications are justified by several reports in the literature. Clinical pharmacists play an increasingly important role, through pharmaceutical analysis, helping to prevent misuse or inappropriate use with these molecules and there by helping to reduce costs.

Disclosure of Interest: None Declared.

\section{HP-PC61}

The Best Possible Medication History for Surgical Patients: Opportunities for Improvement

R. Pham ${ }^{1}$, B. Krug ${ }^{2}$, A. Spinewine 3,* $^{\text {, }}$

${ }^{1}$ Pharmacy, ${ }^{2}$ Quality and Safety Officer, ${ }^{3}$ Clinical Pharmacy, CHU UCL Mont-Godinne, Dinant, Yvoir, Belgium

Background and Objective: To improve the quality of medication histories documented by the anesthetist in the electronic medical record (EMR) for patients coming to the pre-admission clinic.

Setting and Method: Continuous quality improvement project in a Belgian teaching hospital involving two main approaches implemented from 2011 to 2013 : 
$1^{\circ}$ Empowering patients to generate the best preadmission: development, pilot testing and editing of a standardized medication form to be filled in by patients or relatives; at the front: two checklists (routes of administration and anatomic checklist) in order to minimize omissions; at the back: a structured table to list all medications:

$2^{\circ}$ Promoting accurate recording by the anesthetist in the EMR

- Improvement in the structure to report names, dosage, frequency and time of administration in the EMR; dedicated boxes were provided.

- Audit and feedback to the staff

Main outcome measures: $1^{\circ}$ (a) rate of completeness of the patientcompleted medication questionnaire and (b) discrepancies between answers to both checklists and the medications listed, for 2 successive versions of the questionnaire (449 patients coming to the preadmission clinic in June 2012 and 183 in early April 2013).

- $2^{\circ}$ proportion of medications with missing data relative to dosage in the preoperative medication list coded by the anesthetist (EMR) for patients seen in June $2011(n=445)$, June $2012(n=449)$ and early April $2013(\mathrm{n}=184)$.

- $1^{\circ}$ and $2^{\circ}$ discrepancies between patient-completed form and list of anesthetist (EMR)

Results: Patient-completed questionnaire:

- 9 out of 10 patients came to the surgical pre-admission clinic with a patient-completed form (92\% in 2012, $91 \%$ in 2013)

- modifications in the medication form (layout and content) improved the rate of completeness ( $61 \%$ in 2012 vs. $73 \%$ in 2013);

- 1 out of 3 patients omitted to list a medication relative to a box ticked from the checklist (32\% in 2012 and 2013);

- List coded by the anesthetist (EMR):

- the proportion of drugs with missing data relative to dosage decreased (23\% in 2011 vs. $4 \%$ in 2013);

- Discrepancies:

- 1 out of 5 patients had at least one prescribed medication omitted in EMR (17\% in 2012 vs. $21 \%$ in 2013) which was recorded in the patient-completed form: Most of them were "analgesics". Upon discussion, anesthetists reported not considering omission of analgesics as a "clinically relevant" for their practice.

- In $10 \%$ of the patients, the anesthetist found at least one prescribed medication not indicated in the form (10\% in 2012 and 2013)

Conclusions: The lay-out and content of the patient-completed form as well as the structure of the EMR influence completeness.

- Empowering patients is valuable, but careful validation of patient's list remains necessary. The routes of administration and anatomic checklists can be useful for this validation process.

- Completeness of medication history may be influenced by physician's specialty.

Disclosure of Interest: None Declared.

\section{HP-PC62}

Thyroid protection for adults in nuclear medicine: suggestion of a new protocol based on review and survey.

I. Riguet ${ }^{1{ }^{*}}$, D. Galvez ${ }^{1}$, G. El Deeb ${ }^{1}$, N. Rizzo-Padoin ${ }^{2}$, F. Paycha ${ }^{3}$, H. Barreteau ${ }^{4}$

${ }^{1}$ Radiopharmacy, Lariboisière Hospital, ${ }^{2}$ Radiopharmacy, Saint-Louis Hospital, ${ }^{3}$ Nuclear medicine department, ${ }^{4}$ Pharmacy department, Lariboisière Hospital, Paris, France
Background and Objective: Scintigraphies with radiopharmaceutical agents labeled with iodine $123\left(\mathrm{I}^{123}\right)$ require thyroid protection when exploring other organs. Indeed a small amount of free $\mathrm{I}^{123}$ could cause patient's thyroid irradiation. Up to its supply disruption, we used potassium perchlorate. In order to define a new protocol, a literature review was conducted and a survey was submitted to 41 nuclear medicine departments.

Setting and Method: The review consisted in bibliographic research Summaries Products Characteristics (SPC) for both radiopharmaceuticals ( $\mathrm{I}^{123}$-Ioflupane, $\mathrm{I}^{123}$-Iobenguane) and thyroid blocking agents were consulted when available. So we tried to find thyroid blocking agents' pharmacokinetic properties as well as the onset and duration of action.

Regarding the survey, questions focused on the blocking agents used and their posology.

Results: 22 departments responded to our survey (54\%). Two thyroid blocking agents were used: potassium iodide $(\mathrm{KI})(\mathrm{n}=13)$ and Lugol's solution $(n=7)$. We noticed an important diversity of practices among the departments: for one same agent, there were major differences in protocols concerning doses and/or timing of administration. There were only 2 departments that followed exactly the same protocol. Differences were also found within literature, recommendations and SPC.

Moreover, pharmacokinetics data concerning thyroid blocking agents were incomplete. Most of information found was about accidental exposures to radioelements and couldn't entirely explain the protocols used in nuclear medicine.

Finally, the most complete data we found was about KI:

- $100 \mathrm{mg}$ taken at the same instant as the radiopharmaceutical blocks $98 \%$ of the thyroid dose on the same day and $130 \mathrm{mg}$ blocks $95 \% 24 \mathrm{~h}$ later.

- taking $20 \mathrm{mg}$ the following days offers a $90 \%$ protection.

One of the pharmaceutical forms of $\mathrm{KI}$ is $65 \mathrm{mg}$ divided tablets easily available at a low price.

Conclusions: We selected KI for our new thyroid protection protocol which consists of $130 \mathrm{mg}$ of KI taken one hour before radiopharmaceutical agent injection and $65 \mathrm{mg}$ the next day. Several thyroids scintigraphies confirmed its validity as there was no significant thyroid fixation among patients.

Hopefully, some harmonization of practices among nuclear medicine departments will follow.

Disclosure of Interest: None Declared.

\section{HP-PC64}

Medication reconciliation as an efficient tool to prevent medication errors: about 200 patients in geriatric medicine

M. Dufour ${ }^{1, *}$, M. Lottin ${ }^{1}$, S. Philippe ${ }^{1}$, F. Dolard ${ }^{1}$, N. Kadri' ${ }^{2}$, R. Varin ${ }^{1}$, J. Doucet ${ }^{2}$

${ }^{1}$ Pharmacy, ${ }^{2}$ Geriatric Medicine, University Hospital of Rouen, Rouen, France

Background and Objective: Admission and discharge represent hazardous situations for medications errors. At admission, wrong or partial collection of current medication can cause inappropriate or unintentional disruption in medical treatment. Medication reconciliation aims to secure these risky periods, taking into account the patient current treatment in the new prescription. The study aims to evaluate the clinical impact of the medication reconciliation at the patient admission in Geriatric Medecine.

Setting and Method: This study has been realized over 200 patients in a period of 7 months. The comparison between the complete list of a patient's current medication (call systematically the community 
pharmacist) and drugs prescription realized at admission, identified intended or unintended discrepancies. The potential clinical impact of unintended discrepancies has been evaluated independently by a pharmacist and a physician.

Results: Over the 200 patients (mean $83.2 \pm 9.4$ years old), $50 \%$ were home-living without medical help. $65.5 \%$ of patients carried a prescription at the time of admission. Before admission, $8.2 \pm 3.2$ medications were given to patients, and $7.5 \pm 3.2$ were given at admission. 677 intended discrepancies $(32.5 \%)$ were identified, with main causes: drugs substitutions $(29.5 \%)$, current absence of indication $(18.8 \%)$, therapeutic optimizations $(16.7 \%) .118$ unintended discrepancies have been identified, 42 due to prescriptor omission and 76 due to lack of prescriptor information; for an average of 0.59 unintended discrepancies per patient. 58 patients had at least one unintended discrepancie. Most frequent unintended discrepancies were omissions $(76.4 \%)$ and incorrect dose (18.6\%). Most common therapeutic classes were cardiovascular system (23\%), nervous system $(22 \%)$ and alimentary tract and metabolism (16\%). It has been shown by the pharmacist clinical impact analysis that 61 unintended discrepancies $(51.7 \%)$, were likely to have a significant clinical impact and $38(32.2 \%)$ a serious clinical impact. Physician analysis showed 58 unintended discrepancies $(49.2 \%)$ likely to have a significant clinical impact and $20(16.9 \%)$ a serious clinical impact. Both judgements were in line for more than $60 \%$ of the analysis. A positive correlation has been identified between the number of medicine at home and the number of discrepancies.

Conclusions: Our research allowed demonstrating that the medication reconciliation is an efficient method to evaluate and prevent medication errors.

Disclosure of Interest: None Declared.

\section{HP-PC65}

New risks of computerization: a priori analysis of risks (FMECA method) and assessment six months after deployment of computerization in a pediatric service

\section{A. Giroud ${ }^{1, *}$, A. Oufella ${ }^{1}$, V. Duperrin ${ }^{1}$, B. Coret-Houbart ${ }^{1}$ \\ ${ }^{1}$ Pharmacy, R. Ballanger Hospital, Aulnay-sous-Bois, France}

Background and Objective: The computerization of the processes of medicinal care of hospitalized patients, is one of essential conditions of its security. This approach brings major changes in term of organization of the care and also generates new types of errors, that need necessary to be identified and controlled. The pediatric service is particularly at risk. So before the deployment of the software Pharma ${ }^{\circledR}$, a risk assessment was conducted a priori.

The objectives are to identify, analyze, rank and control risks a priori related to the computerization of the pediatric service. A review of adverse events occurred during the first 6 months of deployment was done.

Setting and Method: A group of piloting was established to draw the process and analyze the potential risk according to the FMECA method (failure mode, effects and criticality analysis). The analyzed process includes the computerization applied to medical prescription, pharmaceutical validation, preparation and the individual and personal daily dispensing, and administration. The severity, frequency and detection of these failures were quoted to determine the levels of criticality of the process failures.

Results: We identified 47 stages during the analysis of the process and 63 potential failures. The most critical stage is by far the prescription itself. The main preventive action was the creation of 12 protocols of prescription allowing to optimize the most frequent prescriptions and most at risk, and the training of the prescribers by emphasizing the stages at risk identified. Besides an exhaustive validation of the real time prescriptions is organized. Despite these preventive actions, the assessment carried out highlighted 324 errors of prescription bound to the computing, reported and corrected due to pharmaceutical interventions ( $7.2 \%$ of lines of the prescriptions). The main errors are errors of units (22\%), prescription of forms adults or not adapted (16\%) and forgetting of dosage $(13 \%)$. Another failure of major risk identified is the not corresponding prescription in particular by weight absence or wrong weight.

Conclusions: The a priori risk analysis allowed us to identify more precisely the critical stages of computerization of pediatric service and their relative risks. The implementation of preventive measures allowed to make a safer deployment. The staff training has been highlighted on these new identified risks. A regular review of the pharmaceutical interventions is necessary and establishes a relevant monitoring indicator, a generator of essential corrective measures to secure the medicinal care.

Disclosure of Interest: None Declared.

\section{HP-PC66}

Belatacept in kidney transplant recipients switched from calcineurin inhibitors: results on renal function and safety after 6 months

P. Bardo ${ }^{1, *}$, B. Berdougo ${ }^{1}$, E. Rouimi ${ }^{1}$, P. Grimbert ${ }^{2}$, A. Astier ${ }^{1}$, M. Paul ${ }^{1}$

${ }^{1}$ Pharmacy, ${ }^{2}$ Nephrology Init, Henri mondor Hospital, Créteil, France, Créteil, France

Background and Objective: A phase 2 trial suggested that Belatacept may improve renal function after switching from Calcineurin Inhibitors (CNI)-based therapy and safety profile appeared to be similar to CNI-group.

The switch has been initiated on a group of renal transplant patients in the Nephrology Unit of Henri Mondor Hospital. The objective was to study, after 6 months of use, the efficacy profile and safety of Belatacept in this off-label indication.

Setting and Method: Literature review; retrospective study on patients treated by Belatacept; calculation of the mean and standard deviation or percentage of outcome measures.

Main outcome measures: Calculation of glomerular filtration rates (cGFR) based on the Modification of Diet in Renal Disease formula, rate of survival, graft loss, acute rejection episodes and adverse events, after 6 months of treatment.

Results: 13 kidney transplant recipients received Belatacept switched from a CNI-based regimen since 2012 January. The mean age of the cohort at switch moment was 64 years $[52.5 ; 75.5]$ and mean initial cGFR was $29.1 \mathrm{~mL} / \mathrm{min} / 1.73 \mathrm{~m}^{2}$ [10.4; 47.8]. The duration between switch and transplant was very disparate: from 1 month to 10 years. The population was heterogeneous but they all had a impaired renal function and/or bad CNI tolerance that justified the switch.

Belatacept was given by intravenous infusion at $10 \mathrm{mg} / \mathrm{kg}$ every 2 weeks during 1 month (days 1,14 and 28) and then $5 \mathrm{mg} / \mathrm{kg}$ every month therefore. For early switch (less then 3 months after transplant), the dosage was $10 \mathrm{mg} / \mathrm{kg}$ on days $1,5,14,28$, weeks 2 and 8 , and then $5 \mathrm{mg} / \mathrm{kg}$ every month.

2 patients discontinued Belatacept treatment: one due to an unmanageable CMV infection and the other because of a BK virus infection that needed a low-dose immunotherapy hard to handle with Belatacept. There were no serious adverse events identified. After 6 months of Belatacept treatment, none of the patients had acute rejection episodes or graft loss. One death due to a cardiovascular cause (4 months after switch) was reported inducing a survival rate of $92.3 \%$ at 6 months. 
The mean cGFR at 6 months on 11 patients was $29.5 \mathrm{~mL} / \mathrm{min} /$ $1.73 \mathrm{~m}^{2}[16.4 ; 42.6]$, that is a mean change from baseline of $+0.4 \mathrm{~mL} /$ $\mathrm{min} / 1.73 \mathrm{~m}^{2}$. We observed an improvement of renal function in 6 patients $(54.5 \%)$.

Conclusions: Belatacept used in switch from CNI-based regimen in Henri Mondor Hospital seems to be well tolerated after 6 months without serious adverse effect, acute rejection episode or graft loss. The renal function has not been really improved but we can notice a stabilization, that would be the objective for patients with poor initial function and several adverse effects with CNI-treatment. However, these results needed to be balanced with the high cost of this medical care for the hospital. Disclosure of Interest: None Declared.

\section{HP-PC67}

Is the computerized prescription a key factor in the deployment of pharmaceutical analysis?

S. Kabiche ${ }^{1, *}$, D. Monzat ${ }^{1}$, M. Le Jouan ${ }^{1}$, R. Farinotti ${ }^{1}$, C. Montagnier-Petrissans ${ }^{2}$, P. Le Gonidec ${ }^{1}$

${ }^{1}$ Observatory of Drugs, Medical Devices and Therapeutic Innovations (OMEDIT), ILE De France, Regional Health Agency (ARS), ILE De France, ${ }^{2}$ Medical and administrative Directorate (DMA), AP-HP, Paris, France

Background and Objective: Pharmaceutical analysis of prescriptions is one of the mandatory tasks of hospital pharmacists in France. Its deployment is facilitated by the development of computerized prescription. The Contract of quality use of medicines (CBU) is a French regulatory framework that encourages hospitals to develop both pharmaceutical analysis and computerized prescription. The aims of the study was to analyze the deployment of these two activities in Paris area hospitals, understand the link between them and explain the disparities and obstacles to their development.

Setting and Method: A comparative analysis of annual results of CBU (from 2009 to 2012) in Paris area hospitals was carried out by The Observatory of Drugs, Medical Devices and Therapeutic Innovations (OMEDIT).

Main outcome measures: Rate of beds with computerized prescription, rate of beds that benefited from pharmaceutical analysis.

Results: In 2012, among the 218 Paris area hospitals, $80 \%$ have implemented computerized prescribing system (vs $74 \%$ in 2011). The prescription was computerized for $51 \%$ of beds $(56 \%$ in public setting and $30 \%$ in private setting). The same rate $(51 \%)$ of beds have benefited from pharmaceutical analysis (54\% in public setting and $39 \%$ in private setting). Since 2009 , computerized prescription has tended to develop (34\% in 2009, $36 \%$ in 2010 and $42 \%$ in 2011) while pharmaceutical analysis is stagnating after a strong increase in 2009 (31\% in 2009, $50 \%$ in 2010 and $49 \%$ in 2011). Pharmaceutical analysis of prescriptions is more developed in longterm care units ( $48 \%$ of total beds) compared to acute care units (38\% of total beds). According to the typology of beds, the frequency of pharmaceutical analysis varied greatly (45\% daily, $14 \%$ weekly and $41 \%$ other frequencies).

Conclusions: This analysis has highlighted some differences in both pharmaceutical analysis and computerized prescription deployment. Disparities between public and private settings can be explained by a lower allocation of human and material resources in the private setting. Pharmaceutical analysis was more developed in long-term units care because changing in prescription are less frequent compared to acute care units. Beyond the deployment of computerized prescription, other factors should be taken into account for optimal development of the pharmaceutical analysis.

Disclosure of Interest: None Declared.

\section{HP-PC68}

Impact of a clinical pharmacist in a department of hepatopancreto-biliary surgery and liver transplantation

G. Kreutter ${ }^{1, *}$, M. Fourtage ${ }^{1}$, Y. Nivoix ${ }^{1}$, D. Leveque ${ }^{1}$, P. Bachellier $^{2}$, B. Gourieux ${ }^{1}$

${ }^{1}$ Pharmacy-sterilization department, ${ }^{2}$ Hepato-pancreto-biliary Surgery and liver transplantation, pôle des pathologies digestives, hépatiques et de la transplantation, Hôpitaux Universitaires de Strasbourg, Strasbourg, France

Background and Objective: Clinical pharmacists are contributing to the safe medication use by providing comprehensive management to patients and medical staff. However, less is known on their impact in surgery. The purpose of this prospective study was to document and evaluate the services of clinical pharmacists in a hepatobiliary and pancreatic surgery setting.

Setting and Method: Medication reviews and reconciliation concerning hospitalized patients were performed during a 1-year period (May 2012-April 2013), in a 50-bed hepatobiliary and pancreatic surgery unit of a university hospital. Medications problems, interventions and acceptance rate by the surgeons were recorded by 2 pharmacists.

Main outcome measures: We recorded prospectively pharmaceutical interventions according to the scale established by the SFPC "French Society Of Pharmaceutical Care".

Results: During the study period, 3,829 prescriptions of patients (mean age: 65 years) were analyzed. The pharmacists identified 536 drug-related problems ( $14 \%$ of the prescriptions) primarily related to anti-infective agents (45\%), central nervous system drugs $(15 \%)$, cardiovascular drugs $(13 \%)$ and gastrointestinal tract agents $(11 \%)$. Medications problems $(\mathrm{n}=536)$ included inappropriate medications (19\%), drug omissions (17\%), overdosing (16\%), inappropriate administrations $(12 \%)$, underdosing $(10 \%)$, drug-drug interactions $(8 \%)$. Half $(54 \%)$ of drug omissions were detected with medication reconciliation at admission. Interventions $(n=536)$ led to drug dosing adjustments (22\%), treatment discontinuations and drug additions (19\%), replacement of a drug by another one (15\%), therapeutic drug monitoring (14\%), administration optimization (10\%) alternate routes of administration (1\%). Most (82\%) of the interventions were accepted and implemented by the medical staff.

Conclusions: The integration of clinical pharmacy services resulted in drug specific interventions in $14 \%$ of the prescriptions of hospitalized patients in a hepatobiliary and pancreatic surgery unit. Medication problems mostly concerned anti-infective agents. The intervention acceptance rate by surgeons was high.

Disclosure of Interest: None Declared.

\section{HP-PC69}

Individualised medication adherence program for hypertension patients

U. Hedegaard ${ }^{1, *}$, K. J. Lene ${ }^{2}$, J. Hallas ${ }^{1}$

${ }^{1}$ Institute of Public Health, Clinical Pharmacology and Pharmacy, University of Southern Denmark, Odense, ${ }^{2}$ The Danish Research Unit for Hospital Pharmacy, Amgros I/S, Copenhagen, Denmark

Background and Objective: Approximately 25-50\% of patients with hypertension report non-adherence with their medication with potential adverse outcome. Non-adherence is a multi-dimensional problem occurring for a variety of reasons. Consequently, a successful adherence intervention requires a selection of multiple components to 
address the particular reason for non-adherence. Here, the objective is to describe an adherence program developed for hypertension patients in a clinical pharmacy hospital setting.

Setting and Method: The program was developed for a hospital setting and is used in an ongoing, randomised, controlled study investigating the effect on medication adherence and on new cardiovascular events. The study is carried out at two endocrinology outpatient clinics and one cardiology out-patient clinic at Odense University Hospital. Patients treated for hypertension are randomised to the adherence program $(\mathrm{N}=150)$ or to a control group $(\mathrm{N}=300)$.

The program is delivered by 5 clinical pharmacists, who have been trained in providing the intervention.

The adherence program consists of three components:

1. A medication review focusing on potential adherence-related problems followed by recommendations to the physicians at the clinic.

2. A consultation using narratives and motivational interviewing techniques. The pharmacist uses a short validated questionnaire and a modified version of The Drug Adherence Work-up (DRAW(C) tool + guide to address and take action on the various potential reasons for non-adherence.

3. Follow-up telephone calls one month and six months after inclusion using a short version of the modified DRAWC-tool to uncover potential non-adherence.

The components of the program will be evaluated by the number and type of potential adherence problems identified, the number and type of action taken and pharmacist time spent.

Results: -

Conclusions: An individualised multifaceted adherence program performed by clinical pharmacists has been developed. If the program is efficacious, it may be disseminated to other settings.

Disclosure of Interest: None Declared.

\section{HP-PC70}

Assessment of high-priced systemic antifungal prescriptions in a tertiary care hospital

C. Poulat' ${ }^{1}$, Y. Nivoix ${ }^{1}$, A. Launoy ${ }^{2}$, P. Lutun ${ }^{3}$, P. Bachellier ${ }^{4}$, D. Leveque ${ }^{1, *}$, R. Herbrecht ${ }^{5}$, B. Gourieux ${ }^{1}$

${ }^{1}$ Pharmacy, ${ }^{2}$ Surgical Intensive Care Department, ${ }^{3}$ Medical Intensive Care Department, ${ }^{4}$ Hepatobiliary, Pancreatic and Digestive Surgery Department, ${ }^{5}$ Oncology and Haematology Department, Hopitaux Universitaires de Strasbourg, Strasbourg, France

Background and Objective: The aim of our study was to assess compliance to international guidelines for high-priced and patent protected antifungals (liposomal amphotericin B, caspofungin and voriconazole).

Setting and Method: A retrospective study of those three high-priced systemic antifungals prescriptions was performed from May to July 2012 in three departments: surgical and medical intensive care units and in a hepatobiliary, pancreatic and digestive surgery unit at Hautepierre University Hospital, Strasbourg, France.

Main outcome measures: On reviewing medical charts, the antifungal prescriptions were examined in relation to summary of product characteristics or guidelines for indication, dosage and, when appropriate, antifungal de-escalation. The assessment criteria were classified as appropriate, inappropriate or debatable.

Results: During the study period, 71 treatments were analysed for 56 patients (median age: 60). Treatments were prescribed for prophylactic $(3 \%)$, empirical $(20 \%)$, pre-emptive $(10 \%)$ or targeted therapy $(67 \%)$. Caspofungin accounted for $69 \%$ of prescriptions, followed by voriconazole $(20 \%)$ and liposomal amphotericin B $(11 \%)$. Indications were found to be appropriate in $87 \%$ and debatable in $13 \%$. Dosages were found to be appropriate in $66 \%$, debatable in $10 \%$ and inappropriate in $24 \%$. Prescriptions were deemed inappropriate for the following reasons: lack of dosage adjustment for hepatic function (10 cases) and under- or overdosage by $>25 \%$ of the recommended dose in 7 cases. De-escalation to fluconazole was only done in $40 \%$ of the 28 patients with a stable fluconazole-susceptible Candida infection.

Conclusions: Compared to a first study performed on all systemic antifungals in 2007 in our hospital (1), our study revealed a higher proportion of appropriate indication (65\% in 2007), no inappropriate indication and a slight improvement in appropriate dosing $(62 \%$ in 2007). Nevertheless, there is still room for improvement regarding dosage adjustment and potential de-escalation to fluconazole.

[1] Nivoix Y, Launoy A, Lutun P, Moulin JC, Phai Pang KA, Fornecker LM, Wolf M, Levêque D, Letscher-Bru V, Beretz L, Ubeaud-Sequier G, Herbrecht R. Adherence to recommendations for the use of antifungal agents in a tertiary care hospital. J Antimicrob Chemother. 2012 Oct;67(10):2506-13.

Disclosure of Interest: None Declared.

\section{HP-PC71}

Medication history on admission to the emergency department: Where are the problems and why is it so complex to optimise?

R. Pham ${ }^{1}$, B. Krug ${ }^{2}$, J.-B. Nicolas ${ }^{3}$, C. Claeys ${ }^{4}$, A. Moreau, D. Van Pee ${ }^{6}$, A. Spinewine ${ }^{7 \text {,* }}$

${ }^{1}$ Pharmacy, ${ }^{2}$ Quality and Safety Officer, Institute of Health and Society, ${ }^{3}$ Internal Medicine, CHU UCL Mont-Godinne, Dinant, Yvoir, ${ }^{4}$ Laboratory of pharmaceutical chemistry, Faculty of Pharmacy, Université libre de Bruxelles, Brussels, ${ }^{5}$ Pharmacy, Clinique Saint-Pierre, Ottignies, ${ }^{6}$ Emergency Department, ${ }^{7}$ Louvain Drug Research Institute, Clinical Pharmacy Research Group, CHU UCL Mont-Godinne, Dinant, Yvoir, Belgium

Background and Objective: In our hospital, up to $25 \%$ of inpatients are admitted through the emergency department (ED). Pilot work undertaken by clinical pharmacists highlighted that incomplete medication history frequently originated in the ED.

We aimed to optimize the quality of medication history taken in the ED.

Setting and Method: Continuous quality improvement project in a Belgian teaching hospital from 2011 to 2012;

- a kick-off session was organized with ED staff to introduce the topic and discuss the project (objectives and method)

- a structured medication form to be filled in by patient or relatives on admission and to be used by ED staff was implemented

- feedback on medication discrepancies at baseline was communicated to ED staff

- posters and leaflets were displayed in the waiting room, to raise patient awareness

Main outcome measures: 1 . Identification of medication discrepancies between the medication history recorded by the ED physician as compared to medication history performed by a clinical pharmacist for 77 patients at baseline.

2. Evaluation of clinical relevance of omissions by a multidisciplinary panel.

Results: 1. There were 10.3 discrepancies per patient. The most frequent discrepancies were: omission of time of administration (3.9/ patient), omission of medication (1.9), and omission of dosage (1.9). Omissions mainly involved drugs of the digestive and central nervous systems. 
2. Among patients with at least one omission, $25 \%$ had an omission directly linked to the reason for admission and $52 \%$ had an omission potentially causing harm if not resolved during hospital stay.

A trend toward improvement in medication discrepancies was observed (decrease from 10.3 to 8.7 discrepancies per patient). There was overall no significant reduction in the rate of omissions, which were considered as the most relevant medication discrepancies. We faced many organizational difficulties relative to the distribution and use of the medication form, despite relatively good participation of patients.

Conclusions: Obtaining the best possible medication history in the ED was a challenge due to the presence of many barriers of different types. Clearly defining roles and responsibilities of ED staff in relation to responsibilities of the staff of inpatient unit is an essential step toward optimisation and satisfaction of users.

Disclosure of Interest: None Declared.

\section{HP-PC72}

Managing high dosage diazepam treatment in severe tetanus: a case report

C. Dhelens ${ }^{1, *}$, C. Chapuis ${ }^{1}$, A. Bonadona ${ }^{2}$, P. Bedouch ${ }^{1}$, C. Schwebel ${ }^{2}$, B. Allenet $^{1}$

${ }^{1}$ Department of Pharmacy, ${ }^{2}$ Medical intensive Care Unit, Grenoble University Hospital, Grenoble, France

Background and Objective: Tetanus is an acute fatal infectious disease caused by contamination of wounds from the bacteria Clostridium tetani, or the spores they produce. Despite immunization strategy, tetanus remains a health problem in industrialized countries, with $30 \%$ of case fatality rate. Mortality results from respiratory failure, cardiovascular collapse or autosomic dysfunction. Treating muscle spasm and rigidity by high dose sedatives, such as diazepam (Valium ${ }^{\circledR}$ Roche), avoid potential life-threatening complications. Those high doses of intravenous (IV) diazepam cause stability and compatibility issues. We report here the management of very high dose diazepam treatment in a severe tetanus case.

Setting and Method: A non-immunized 80 year-old woman was hospitalized in an 18-bed intensive care unit (ICU) in our 2000-bed university hospital for severe tetanus with trismus, muscle spasms, stiffness of the neck, leading to acute respiratory failure. Management included tetanus antitoxin, immune globulin, antibiotics, neuromuscular blocking agent, mechanical ventilation, supportive nursing care, baclofen, and sedatives including IV continuous undiluted diazepam titration $(5 \mathrm{mg} / \mathrm{ml})$ up to $10 \mathrm{mg} / \mathrm{kg} / 24 \mathrm{~h}$. Upon our onsite pharmaceutical order review, no Y-site injection incompatibilities were detected, whereas physical incompatibilities (white precipitate if concentration $>0.25 \mathrm{mg} / \mathrm{ml}$ ) was described for undiluted diazepam. We suggested doing at least a 2:3 dilution. Unfortunately, clogging of the central venous catheter occurred without thrombophlebitis, leading us to higher dilutions as soon as clinical parameters improved. Four weeks after treatment, the patient recovered without further complications.

Results: Recommendations about injectable drugs incompatibilities are subject to interpretation due to the uniqueness of each clinical situation. In this case, we had to manage unusually high dosages. We were also constrained by over hydration risk and the volume of infusion had to be strictly restricted, despite the expected incompatibility. Solution compatibility studies regarding diazepam infusion are contradictory and should be cautiously interpreted. Some authors highlighted that physical incompatibility was related to the solubilizing agent (sodium benzoate) in diazepam formula containing this agent, more than diazepam itself.

Conclusions: Preventing incompatibilities issues in severe tetanus spasms with high dosage diazepam is difficult and must take into account patients' risk-benefit ratio between a suitable therapeutic strategy and instructions about drug administration.

Disclosure of Interest: None Declared.

\section{HP-PC73}

Managing carboxypeptidase rescue after methotrexate treatment

A. S. Promis, ${ }^{1, *}$, C. Di Fiore-Faye ${ }^{1}$, A. Pages ${ }^{1}$, F. Farbos ${ }^{1}$, C. Vinson ${ }^{1}$, S. Perriat ${ }^{1}$, A. Grand ${ }^{1}$, J.-M. Canonge ${ }^{1}$

${ }^{1}$ Oncology Clinical Pharmacy Unit, CHU DE Toulouse, Toulouse, France

Background and Objective: Carboxypeptidase G2 (CPG2) is an enzyme that hydrolyses methotrexate to inactive metabolites, indicated for treatment of toxic plasma methotrexate concentrations. This emergency alternative is managed by hospital guidelines. Two criterias permit carboxypeptidase single use within $96 \mathrm{~h}$ : methotrexate plasma concentration $>10^{*} 10^{-6} \mathrm{~mol} / \mathrm{L} 48 \mathrm{~h}$ after administration or/and increase of creatinine levels by $150 \% 24 \mathrm{~h}$ after infusion.

The aim of this study was to evaluate patients' response and professional practices regarding to the hospital guidelines to assess their impact.

Setting and Method: We consulted patient medical files in seven units (pediatric, reanimation, neurology and hematology units) with retrospective collection of data for those who received carboxypeptidase infusion between January 2012 to April 2013. We collected the following information: patient data, chemotherapy protocol, methotrexate and carboxypeptidase posology, methotrexate plasma levels, renal function assessment, prevention methods (folinate, overhydration, alkalinization).

Main outcome measures: Methotrexate plasma concentration, creatinine levels, administration data.

Results: 181 patients received methotrexate and nine of them (5\%) were treated with carboxypeptidase (average age: 51, sex ratio: 2). Eight patients received a single dose of 50UI per Kg. Seven patients $(78 \%)$ received the antidote within $96 \mathrm{~h}$. Eight patients $(89 \%)$ developed acute renal failure (ARF) (creatinine levels $>1.5^{*}$ basal level) versus four patients had methotrexate levels $>10 \times 10^{-6} \mathrm{~mol} / \mathrm{L}$. All patients with methotrexate levels $>10 \times 10^{-6} \mathrm{~mol} / \mathrm{L}$ had also ARF. Only one patient received this medication whereas the two criterias were not fulfilled.

The mean time to recovery renal function was 23 days (8-40) after administration of methotrexate. All the patients receiving carboxypeptidase continued their initial chemotherapy treatment.

Conclusions: According to these results, it seems to be necessary to transmit recommendations to prescribers in order to promote experience return and improve patient safety. Our experience confirms that the role of the hospital pharmacist is important to ensure proper use of carboxypeptidase.

Disclosure of Interest: None Declared.

\section{HP-PC74}

Evaluation of pharmaceutical analysis based on criteria targeting high-risk prescriptions: a multicenter approach in French hospitals

E. Jean-Bart ${ }^{1, *}$, L. Vadot ${ }^{2}$, M. Bourdelin ${ }^{1}$, R. Puech ${ }^{3}$, J. Liehn ${ }^{2}$, B. Leroy ${ }^{2}$, S. Coursier ${ }^{1}$, E. Pont ${ }^{3}$, J. Coutet ${ }^{2}$, H. Bontemps ${ }^{1}$

${ }^{1}$ Service Pharmacie, L'Hôpital Nord Ouest, Villefranche-sur-Saône, ${ }^{2}$ Service Pharmacie, Centre Hospitalier William Morey, Chalon-surSaône, ${ }^{3}$ Service Pharmacie, Centre Hospitalier Pierre Oudot, Bourgoin-Jallieu, France 
Background and Objective: In France, for hospital prescriptions analysis, the legislation recommends for pharmacists to lead their analysis toward risk drugs and risk patients. Due to the large amount of prescriptions to be analyzed, it may be necessary to select the highest risk requirements. In three general hospitals, an analysis based on risk criteria, was implemented.

The aim of this study was to evaluate the activity of pharmaceutical analysis based on criteria targeting high-risk prescriptions.

Setting and Method: This multicenter study was conducted from January to May 2013. Daily, a computer application in relation to the biology lab detected criteria and edited a list of patients. Criteria used to identify patients at risk were: serum potassium $>5.2 \mu \mathrm{mol} / \mathrm{L}$, serum potassium $<3 \mu \mathrm{mol} / \mathrm{L}$, creatinine clearance (Cockroft) $<30 \mathrm{ml} / \mathrm{min}$ and INR $>4$. A prescription analysis of these patients was performed (level 2 analysis according to the French Society of Clinical Pharmacy).

Main outcome measures: The information collected was: number of detected criteria, type of pharmaceutical interventions (PI), classes of drugs concerned.

Results: In total, 6,568 criteria were detected. The average age of patients was 78 years old. At least one PI was performed for $25.3 \%$ of prescriptions with criteria. $60.3 \%$ of PI were related to criteria. PI for prescriptions with risk criteria potassium, INR and creatinine clearance, were linked to the criteria in respectively $65 \%, 65.4 \%$ and $58.1 \%$ of cases. The most common PI were related to a supratherapeutic dose $(21.1 \%)$, a contraindication $(16.7 \%)$ or an adverse event $(12.8 \%)$. The most implicated drugs by PI related to risk criteria were ionic supplements (14.9\%), ACE inhibitors and sartans $(14.5 \%)$, antibiotics $(10.9 \%)$ and anticoagulants $(10.6 \%)$.

Conclusions: Pharmaceutical analysis based on criteria targeting high-risk prescriptions can help the pharmacist to identify risk situations with an important rate of PI in relation to criteria, and improve patient safety. This method could be simply established. However, the activity is presently focused only on biological criteria. An approach based on risk drugs would improve the method. For example, analysis of all initiated prescriptions of a new oral anticoagulant will be evaluated.

Disclosure of Interest: None Declared.

\section{HP-PC76}

\section{Adequacy of medication in community-dwelling elder patients}

I. Gozalo ${ }^{1, *}$, M. Serra-Prat ${ }^{2}$, L. Campins ${ }^{1}$, E. Palomera ${ }^{2}$, D. López ${ }^{1}$, I. Ruíz ${ }^{1}$, M. Camps', V. García ${ }^{1}$, C. Agustí1

${ }^{1}$ Hospital Pharmacy Department, ${ }^{2}$ Research unit, Hospital de Mataró, Mataró, Spain

Background and Objective: Polypharmacy and inappropriate medication use is a problem in elderly patients, who are more likely to experience adverse effects from multiple treatments.

Our objective was to assess the adequacy of pharmacotherapy in polimedicated community-dwelling elder patients.

Setting and Method: Cross-sectional study of a random sample of elders of the community (not institutionalized) 70 years or older who were receiving 8 or more medications chronically.

A clinical pharmacist re-evaluated all prescribed drugs with the help of STOPP \& START, Beers criteria, Garfinkel algorithm and the clinical practice guidelines.

Main outcome measures: The main outcome measures were the number of clinical pharmacist's recommendations, the degree of acceptance of these and the analysis of the main drugs involved.

Results: 252 patients were included in the study, the mean baseline drug was 10.79 (SD 2.52) medications per patient, the mean age was $79.16(5.5)$ years and the $60.3 \%(n=152)$ were women.
The total number of drugs analyzed was 2,709 . The most prescribed drug groups were antihypertensive drugs (478 drugs, $17.7 \%$ ), non-steroidal anti-inflammatory drug (NSAID)-other analgesics (231, $8.5 \%)$, drugs for acid related disorders $(229,8.5 \%)$, lipid modifying agents $(187,6.9 \%)$ and antithrombotics (172. $6.3 \%)$.

The pharmacist made a recommendation in the $25.43 \%(n=689)$ of the prescriptions because considered inappropiate; these recommendations were accepted by $75 \%$ of cases.

Pharmacological group with more pharmaceutical recommendations was drugs for acid related disorders ( $54.15 \%$ prescriptions), followed by benzodiazepines $(28.57 \%)$, osteoporosi therapy $(27.83 \%)$, NSAIDother analgesics $(25.97 \%)$, antidepressants $(22.6 \%)$, lipid modifying agents $(20.86 \%)$, antithrombotics (19.77\%), other cardiovascular drugs $(18.12 \%)$, oral antidiabetics and insulin $(17.2 \%)$, antihypertensives $(15.27 \%)$ and corticosteroids (13.4\%).

Conclusions: A quarter of the prescripcions analyzed were considered potentially inappropriate for the clinical pharmacist.

Pharmaceutical recommendations were accepted by $75 \%$.

The drugs for acid related disorders were the group who had the highest number of potentially inappropriate prescriptions (more than half). Disclosure of Interest: None Declared.

\section{HP-PC77}

Implementation of a monitoring program to increase medication safety in patients with chronic kidney disease

M. Puig-Bartrina ${ }^{1, *}$, N. Sala-Vilajosana ${ }^{2}$, P. Modamio', A. Vilaseca-Tomas ${ }^{2}$, C. F. Lastra ${ }^{1}$, E. L. Mariño ${ }^{1}$

${ }^{1}$ Clinical Pharmacy and Pharmacotherapy Unit. Department of Pharmacy and Pharmaceutical Technology, Faculty of Pharmacy. University of Barcelona, Barcelona, ${ }^{2}$ Department of Pharmacy, Sant Joan de Deu Hospital, Manresa, Barcelona, Spain

Background and Objective: Patients with chronic kidney disease (CKD) are more likely to experience adverse drug events. The objective was to establish and evaluate a monitoring and dose adjustment program for patients with CKD. An additional objective was to ensure that program development was based on protocol that optimized available resources and thus allowed for continued use in patient intervention strategies.

Setting and Method: Prospective study focused on pharmacist intervention. The study was conducted in a district general hospital during two months. Patients with all stages of CKD were initially included. The stage of CKD was determined by serum creatinine and glomerular filtration rate (GFR) values calculated according to the MDRD formula. Medications requiring dose adjustments for patients with CKD were selected based on hospital guidelines and after entered into a patient monitoring computer program. Using estimated GFR values, the program detects patients at greater risk for adverse drug events due to impaired renal function. Daily analyses of such patients would thus aid pharmacists to alert the prescribing physician in order to proceed with the proper intervention. The computer program was adjusted for the second month of the study to detect only patients in the severe stages of CKD.

Main outcome measures: Pharmacist interventions and physician acceptance rate of interventions.

Results: During the first month, 24 of the 132 selected patients received pharmacist intervention $(18 \%) ; 62 \%$ being female with an average age of $80 \pm 12.5$ years. The percentage of patients detected by the program who received intervention was $50 \%, 38 \%$ and $12 \%$ in CKD stages 3, 4 and 5, respectively. The second month of program evaluation resulted in 24 of 62 selected patients receiving pharmacist intervention (39\%); $71 \%$ being female with an average age of $79 \pm 8.5$ years. This phase of the study revealed the percentage 
receiving intervention to be $12 \%, 46 \%$ and $42 \%$ for patients with CKD stages 3, 4 and 5, respectively. The percentage of pharmacist interventions accepted by the physician was $91 \%$ in the first month and $95 \%$ in the second month. No differences were found in the classes of medications intervened in the first and second month, and were the following: antibiotics (28\%), antithrombotics (19\%), lipidlowering agents $(16 \%)$, hypoglycemic agents (13\%), analgesics $(9 \%)$, cardiac glycosides $(6 \%)$ and others $(9 \%)$.

Conclusions: The program's accurate detection of CKD patients at increased risk for adverse drug events was essential in allowing for proper intervention. The high percentage of pharmacist interventions accepted by the physician also attests to the value of this program and its ability to aid the pharmacist in identifying at-risk patients. The study results suggest the implementation and continued improvement of this patient-monitoring program to be a promising system to improve health outcomes in patients with CKD.

Disclosure of Interest: None Declared.

\section{HP-PC78}

\section{Validity and representativity of a national DRP database}

\section{J. Kjeldsen 1,*, T. Birkholm ${ }^{2}$, H. Fischer ${ }^{1}$, T. Graabæk ${ }^{3}$,} K. P. Kibsdal ${ }^{4}$, T. Truelsh $ø j^{5}$

${ }^{1}$ The Danish Research Unit of Hospital Pharmacy, AMGROS I/S, Copenhagen, ${ }^{2}$ The Hospital Pharmacy, Region Zealand, Roskilde, ${ }^{3}$ The Hospital Pharmacy, Region of Southern Denmark, Esbjerg, ${ }^{4}$ The Hospital Pharmacy, North Region Denmark, Aalborg, ${ }^{5}$ The Hospital Pharmacy, Central Denmark Region, Skejby, Denmark

Background and Objective: Identification of drug related problems (DRPs) is an essential part of conducting medication reviews; hence clinical pharmacists at Danish hospitals have requested a national database to ensure standardized, electronic documentation of DRPs. In July 2010, the DRP-database was introduced as a tool to ensure quality assurance and information sharing. It is, however, uncertain to which extent the DRP-database is used, and whether it is used consistently among the hospital pharmacies. The objective of the study was to explore the validity and representativity of the DRP-database nationally.

Setting and Method: The DRP-database is available to all clinical pharmacists at hospitals in Denmark, but the use is voluntary. The validity of the DRP-database was explored by categorization study, where 24 cases were categorized and documented in the DRP-database by current users. A golden standard was developed by the project group. To evaluate the representativity of the DRP-database, an electronic survey containing questions regarding current and previous use of the DRP-database was provided to all locations using the DRPdatabase.

Main outcome measures: 1: The $\overline{\mathrm{k}}$-values of cases categorized by clinical pharmacists and the project group, 2: The proportion of identified DRPs documented in the DRP-database.

Results: Of the 41 invited clinical pharmacists, 34 (83\%) provided data for the categorization analyses. The results showed $17 \overline{\mathrm{k}}$-values between 0.61 and 0.80 (substantial agreement) and 17 between 0.81 and 1.00 (almost perfect agreement). However, some cases had been recorded multiple times to indicate that more than one ATC code was related to the case. The electronic survey was completed by 15 locations $(100 \%)$, and the results showed that medication reviews were conducted at 12 locations. The majority of the DRPs identified were consistently documented in the DRP-database, and 8 locations documented $100 \%$.

Conclusions: The categorization study showed a fair validity with "substantial" and "almost perfect" agreements between categorizations by the clinical pharmacists and the project group. The representativity revealed that the majority of identified DRPs are documented in the DRP-database. The findings indicate that data in the DRP-database are comparable across the country, and national analyses may be conducted to explore development areas for common interest. Disclosure of Interest: None Declared.

\section{HP-PC79}

Evaluation of the impact of a daily pharmacist presence in a rheumatology unit

\section{P.-O. Perichon ${ }^{1}$, I. Griffoul ${ }^{2}$, D. Mulleman ${ }^{2}$, X. Pourrat ${ }^{1 \text {,* }}$}

${ }^{1}$ Pharmacy, ${ }^{2}$ Rheumatology, CHRU Trousseau, Chambray-lès-Tours, France

Background and Objective: Professional cooperation at hospital between physicians and pharmacists is essential to optimize drugs prescription and to avoid medication errors. Furthermore, pharmacist's presence in the ward at full time let the integration with all the medical team (residents, physicians, nurses). Communication with physicians and integration in the unit is also essential to foster the acceptation of pharmacist interventions. The aim's study was to evaluate the importance of a full time pharmacist on the ward in order to intercept medication problems as soon as possible.

Setting and Method: Six months prospective and observational study in a 23 beds rheumatology unit (full hospitalization); daily presence (except week-ends) of the resident pharmacist or the senior pharmacist during the medical round; full pharmaceutical analysis of the prescriptions: medication reconciliation at admission, biological and clinical parameters, adequacy between medication and diseases taking care of pathophysiology, posology, contraindications, undesirable effects and drug-drug interactions with a on-line system [Theriaque $\left.{ }^{\circledR}\right]$. Main outcome measures: Total number of pharmacist interventions (PI) discerning PI made by pharmacist and answers to the physicians' questions; average length of stay.

Results: 464 patients have been tracked, 4,051 prescriptions' lines have been analyzed and 179 PI realized (4, $4 \%$ of total prescriptions) 116 patients $(25 \%)$ had at least 1 PI. $151(84,4 \%)$ PI were from the pharmacist to the physicians and $28(15.6 \%)$ were questions asked by physicians. Among those from pharmacist to physicians, 134 $(88.7 \%)$ have been accepted and they have led a prescription modification. Average length of stay during the study period was 6.7 days vs 8.9 days last year and 7.8 days two years ago.

Conclusions: Results of the study reflect the importance of the pharmacist's presence in a rheumatology unit and the interest to patient and medical team. Even if it's a time-consuming activity for pharmacists, the profit is real and it leads to decrease of errors medication. There is an important variation of the average length of stay between years, the observed drop is probably multifactorial.

Disclosure of Interest: None Declared.

\section{HP-PC80}

Securing hyperthermic intraperitoneal chemotherapies system after a medication error
J. Daupin ${ }^{1, *}$, Y. Boukari ${ }^{1}$, J. Fillon ${ }^{1}$, A. Houhou ${ }^{2}$, I. Debrix ${ }^{1}$, V. Loi ${ }^{3}$, M. Fartoukh ${ }^{2}$, F. Baud ${ }^{1}$
${ }^{1}$ Pharmacy Department, ${ }^{2}$ Anaesthetics and Intensive Care Department, ${ }^{3}$ Abdominal and Pelvic Surgery Department, Tenon Hospital, 4 rue de la Chine, 75020 Paris, France

Background and Objective: Hyperthermic IntraPEritoneal Chemotherapy (HIPEC) is performed in peritoneal carcinomatosis 
with high doses of oxaliplatin. A case of accidental administration of oxaliplatin by the intravenous route occurred in a patient scheduled for HIPEC in our institution leading to a 4 weeks stay in intensive-care. We report the analytic process and corrective measures that were developped to avoid the recurrence of such a near-miss event.

Results: Independent medical risk management experts' group has analyzed the causes and established the contributing factors of this administration error using the ALARM method (Association of Litigation And Risk Management). The main causes were the simultaneous presence of intraperitoneal oxaliplatin and intravenous $5 \mathrm{FU}$ in the operating room, the non-reading of infusion bags labels by the nurse anaesthetist (NA), the lack of report of the prescription in the medical record and the lack of check-back of drug administration. Contributing factors have been classified as 1/related to patient: severity of the disease that has reinforced the NA concerning the presence of 2 infusion bags 2/related to process: none specific HIPEC process procedure 3/related to teams organization: lack of information sharing between the pharmacy, operating room nurses (ORN) and NA, no systematic check-back between the teams 4/individual factors: lack of education on anticancer drugs toxicity for nurses. A multidisciplinary workshop has set up corrective measures and procedures securing HIPEC process:

- isolation of the delivery routes of preparations: HIPEC/other drugs - in order not to mix oxaliplatin for HIPEC with injectable drugs in the same operating room, receipt and check of the preparations firstly by the NA for intravenous drugs and secondly by the ORN for intraperitoneal oxaliplatin only, after installation of HIPEC equipment

- double reading of products labels before administration

- traceability of checks and of prescriptions in medical records

- educative notice on HIPEC process, drugs toxicity and precautions to be taken

- trainees traceability

An evaluative phase is ongoing.

Conclusions: Through a feedback with a root causes analysis, an entire drug use process has been reassessed from a single medication error. This collective approach allows to manage the risk and to improve continuously the medication use system and the quality of care.

Disclosure of Interest: None Declared.

\section{HP-PC81}

Analysis of a medication error with high dose methotrexate during a week-end

\section{A. Delpeuch ${ }^{1, *}$, P. Simonet ${ }^{1}$, D. Leveque ${ }^{2}$, B. Gourieux ${ }^{2}$, R. Herbrecht ${ }^{3}$}

${ }^{1}$ Pharmacy, Hôpitaux Universitaires de Strabourg, ${ }^{2}$ Pharmacy, ${ }^{3}$ Haematology, Hôpitaux Universitaires de Strasbourg, Strasbourg, France

Background and Objective: Methotrexate is an antifolate agent primarily eliminated by the kidneys and that is used at high dose $\left(>1 \mathrm{~g} / \mathrm{m}^{2}\right)$ in the treatment of certain types of cancer. Various procedures are applied to limit toxicity including the prevention of drugdrug interactions that might delay the elimination of the antifolate. We report and analyze a case of toxicity to high-dose methotrexate in a context of a preventable interaction with piperacillin/tazobactam occurring in a leukemic patient during a week end.

Setting and Method: A hospitalized 47-year-old female with acute lymphoblastic leukemia was received on Wednesday $4.8 \mathrm{~g}$ of methotrexate by venous perfusion with rescue by folinic acid $(50 \mathrm{mg}$ four times a day).

Main outcome measures: Methotrexate delayed elimination occurred $48 \mathrm{~h}$ (Friday) after administration: serum concentration was $2.64 \mu \mathrm{mol} / \mathrm{L}$ at $48 \mathrm{~h}(\mathrm{~N}<1.5 \mu \mathrm{M})$ and $2.22 \mu \mathrm{mol} / \mathrm{L}$ at $72 \mathrm{~h}$ $(\mathrm{N}<0.15 \mu \mathrm{M})$. The patient had severe mucositis and headaches but the renal function was not altered. The daily dose of folinic acid was increased to $200 \mathrm{mg}$ four times a day. Toxic methotrexate serum concentrations were sustained for the week end and were only abated (i.e. fell below $0.07 \mu \mathrm{mol} / \mathrm{L})$ on Monday $(108 \mathrm{~h}$ after administration of methotrexate) with the discontinuation of piperacillin/tazobactam by the clinical pharmacist. Toxicity and delayed elimination of methotrexate were imputable to the co-administration of the antibacterial agent initiated at the beginning of the week end (42 $\mathrm{h}$ after methotrexate perfusion), a known interaction in the haematology unit.

Results: Several factors were identified: prescription of piperacillintazobactam by a resident working outside the haematology unit, absence of the clinical pharmacist during the week-end and the absence of a unique prescription gathering both the chemotherapy and the other medications. Actions have been implemented to avoid this preventable medication error: information of all the physicians of the department on the risks of interactions with methotrexate, better transmissions regarding administrated chemotherapies before the week end and a unique document that include all prescription drugs.

Conclusions: This medication error due to a preventable drug-drug interaction underlines the risks of prescribing during the week end. Disclosure of Interest: None Declared.

\section{HP-PC82}

What tools to improve the undernourished patients' care?

M. Bourges ${ }^{1, *}$, J. Guevin ${ }^{1}$, F. Panisset ${ }^{2}$, R. Ettwiller ${ }^{1}$, F. Gounon ${ }^{1}$, M. Marchand ${ }^{1}$, R. Pascale ${ }^{1}$, D. Hardelin ${ }^{1}$, M.-A. Aka-adjo ${ }^{3}$

${ }^{1}$ Pharmacy, ${ }^{2}$ Quality management, ${ }^{3}$ Endocrinology, Hopitaux Du Leman, Thonon, Les, Bains, France

Background and Objective: The efficiency and the tolerance of parenteral nutrition (PN) depend on the quality of their prescription and the rigor of their follow-up care. With the cooperation of the Nutrition and Alimentation Liaison Committee and the risks gestion committee, a clinical audit has been carried out on PN practices. The objective was to evaluate the detection of underfed patients and their care at the hospital.

Setting and Method: In 2012, 30 in-patients records from 9 units were assessed for a week.

Main outcome measures: Five criteria were evaluated then: the pertinence of the instructions given, the nutritional assessment, the initial biological check-up, the prescription and the follow-up.

Results: The indication of PN was well respected: $40 \%$ of the patients were suffering from a nonfunctional digestive tract, $60 \%$ had first been given enteral feeding. In $60 \%$ of records, the loss of weight had not been assessed for the last six months. An initial biological check-up was done, but $66 \%$ were incomplete. As for the prescription, energy and protein supplies were well adapted to the estimated needs, as well as the addition of vitamins and trace elements (75\% of prescriptions). The method of administration was filled in half the time. $40 \%$ of the prescribers specified the route of administration and $16 \%$ prescribed the drugs available at the hospital, specifying the presence or not of electrolytes and the volume. As for the monitoring of infectious risks, it was well done ( $90 \%$ ), as well as a regular body weighing (76\%) and the blood electrolytes check-up (76\%). The 
monitoring of the water balance (33\%), the glycemia (50\%) and the hepatic function tests $(39 \%)$ had to be improved.

Conclusions: This study demonstrated the pertinence of the instructions given, but also the nonconformity with the recommendations of the French Society of Enteral and PN. Actions were carried out: training courses, protocols. A nominative prescription protocol for PN has been led so as to help prescribers detect and take care of undernourished patients. Two months later, $75 \%$ of the prescribers used this tool. Within a year, a new audit will determine if these corrective actions have been useful to improve the quality of undernourished patients' care.

Disclosure of Interest: None Declared.

\section{HP-PC83}

Development of an appropriate medications list for older adults

\section{S. Hannou ${ }^{1, *}$, B. Michel ${ }^{1}$, M.-C. Rybarczyk ${ }^{1}$, A. Rousseau ${ }^{1}$}

${ }^{1}$ Regional Health Authorities, Strasbourg, France

Background and Objective: In order to improving patient care by optimizing drugs prescription, OMEDIT led a study to develop a drugs list containing appropriate medications for older adults. The purpose was to offer a tool to support the skilled nursing facility to build its own drugs formulary.

Setting and Method: The study took place from December 2011 to April 2012. At start, a preliminary drugs list was done based on drug formularies available in some Alsace nursing homes. Then, the preliminary list was submitted to a panel of 53 experts with international origin and recognized expertise (geriatricians, general practitioners, hospital and community pharmacists, academics, pharmacovigilance scientist...). Their opinions were collected through a two rounds Delphi process to reach a consensus.

Main outcome measures: The experts rated medications for appropriateness in elderly according to five-point Likert scale, from level 1 (definitely appropriate) to 5 (strongly inappropriate). Inappropriate medication was defined on arguments of ineffectiveness, low benefit/ risk ratio when a safer alternative is available, use in specific medical conditions or unsuitable pharmaceutical form.

Results: In the first round, among 338 drugs, 246 (73\%) were considered as appropriate by the experts, $28(8 \%)$ inappropriate and 64 $(19 \%)$ were submitted again in the second round. Then, $32(50 \%)$ were considered as inappropriate and $32(50 \%)$ remained without consensus. Overall, 246 drugs evaluated as appropriate for elderly constitute the final list.

Conclusions: This study pointed out sixty drugs commonly prescribed to older adults but to be discarded according to the evaluation. Our list is a tool at disposal of nursing homes in order to define by further selection through collaborative practices of all professionals caring for residents their own drugs formulary.

Disclosure of Interest: None Declared.

\section{HP-PC84}

Satisfaction study about pharmaceutical consultation and evaluation of drug therapy knowledge

\author{
A. Grave ${ }^{1, *}$, S. Planès ${ }^{1}$, D. Charlety ${ }^{1}$, L. Foroni ${ }^{1}$, P. Bedouch ${ }^{1,2}$, \\ B. Allenet ${ }^{1,2}$ \\ ${ }^{1}$ Pharmacy Department, Grenoble University Hospital, ${ }^{2}$ Centre \\ National de la Recherche Scientifique, TIMC-IMAG UMR \\ 5525/Themas, CNRS, Grenoble, France
}

Background and Objective: To evaluate the impact of a clinical pharmacist consultation on satisfaction, compliance and knowledge about their treatment of oncology patients.
Setting and Method: 29 inpatient and outpatient in an oncology ward were seen by a clinical pharmacist in September and October 2012 for their first chemotherapy. The consultation was about chemotherapy, side effects, treatments at home and the pharmacist handed a reminder chart if necessary.

Coming back for their next chemotherapy, patients had to evaluate the pharmacist's contribution, their satisfaction and their compliance, with a self-administered questionnaire. Then, the pharmacist asked a few question to evaluate patients' knowledge about their treatment.

Compliance rate was based on the Morisky-Green test.

Results: $65.5 \%$ of patients are women and the average age is 55 [ \pm 10.5$]$. Cancer diagnoses included 8 hematologic diseases and 21 solid cancers. A reminder chart was provided by the pharmacist to $72.4 \%$ of patients. Patients' treatments were oral chemotherapy (14\%), treatment to prevent infectious disease (45\%), to prevent nausea and vomiting $(83 \%)$, corticoids $(93 \%)$ and growth factors (31\%). All patients were satisfied with the interview they had with the pharmacist and considered the interview useful. A majority of patients $(96,5 \%)$ said the pharmacist visit improved their way to take medications and ability to manage drugs' side effects. All patients who received a reminder chart were satisfied. They considered it understandable and useful both to understand prescription and for their organization. Most of patients $(96.5 \%)$ have either a "very good" or a "quite good" compliance.Factors identified as influencing treatment compliance were in order of importance the number of pills, delay to take the medication, omission to take pills the day before and memory loss. A majority of patients (56\%) know why all of their drugs were prescribed for, but it's more difficult for them to know exactly how to take their drugs (40\%).

Conclusions: Development of clinical pharmacy consultation in oncology ward is an emerging practice. This study shows that patients are highly satisfied with the pharmacist consultation and that compliance seems to be associated with a better level of understanding. A large study is necessary to confirm the result of this pilot study. Disclosure of Interest: None Declared.

\section{HP-PC85}

\section{Impact of pharmaceutical interventions in a French regional} hospital

\section{G. Belhabib ${ }^{1, *}$, I. Lariviere ${ }^{1}$, V. Di Marco ${ }^{1}$ and pharmacy Hospital Gonesse}

${ }^{1}$ Pharmacy, Gonesse Hospital, Gonesse, France

Background and Objective: In accordance with the recommendations of health authorities, a computerized prescription system was introduced in our hospital in order to secure drug circuit. The purpose of our work is to evaluate the quality of patient management through the pharmaceutical prescription analysis.

Setting and Method: Prospective study was performed from April to June 2013 in gastroenterology (26 beds), neurology (25 beds), respiratory disease (30 beds), infectious disease (26 beds) and orthopaedic services (40 beds), in which a daily dispensation is implanted.

Each prescription was analysed with patient's case and biological results. Pharmaceutical interventions were conveyed either by phone or directly by note on cristal@net software.

Main outcome measures: Pharmaceutical activity (number of analysis and interventions), nature of problems, drugs involved and their outcomes were collected.

Results: A total of 2,279 prescriptions were analysed and 373 pharmaceutical interventions (16\%) were made, concerning drugs choice (42\%), drugs adjustment (22\%), administration optimisation (17\%), computer related problem $(7.5 \%)$, drug-drug interactions $(3.5 \%)$ 
and others $(8 \%)$. Two hundred and fourty-two of them were accepted $(65 \%)$. Among the four main groups of interventions, the higher rate of acceptance was obtained in the resolution of computer related problem $(93 \%)$.

Drugs choice has also a good acceptance (66\%), in which formulary drugs hospital respect, antibiotics guidelines (fluoroquinolones), high-risk drugs (vitamin $\mathrm{K}$ antagonists and potassium) were focused in respectively $79 \%, 100 \%$ and $62 \%$. Lower rate were found for administration optimisation (54\%) and dose adjustment (56\%).This last result could be explained by the few rate of overdose acceptation (49\%), whereas the efficiency of our action on adjustment in renal impairment $(64 \%)$.

Conclusions: This study confirms the beneficial role of multidisciplinary collaboration to enhance the patient management. Pharmacists have a major impact by the large number, the pertinence and the high rate of acceptance of interventions made. However, we have to solve the problem of prescriptions irrelevant to the formulary drug hospital, which would limit an automating drug delivery (ongoing project for the next months).

Disclosure of Interest: None Declared.

\section{HP-PC86}

Evaluation of the interest and the organization of a Medication Reconciliation activity in a psychiatric hospital

P. Feistl' ${ }^{1}$, M. Colombe ${ }^{1, *}$, L. Cochard ${ }^{1}$, A. Martin ${ }^{1}$, C. Gabriel-Bordenave ${ }^{1}$, C. Roberge ${ }^{1}$

${ }^{1}$ EPSM Caen, Caen, France

Background and Objective: Since February 2012, a Medication Reconciliation (MR) program is performed in two services of our psychiatric hospital: EPSM de Caen, in France, to enhance reliability of the medication prescription at inpatient admission. Pharmacist students lead initially an investigation to find various information sources and collect these data on a survey form. Survey is considered ended when we have at least 2 corresponding sources for each line of drug. Then, students fill a MR form, validated by physicians and put it in patient file.

Setting and Method: Data collection documents were designed, in accordance with criteria and indicators chosen. Data analyses were carried out via Excel. Some can be compared with the equivalent data collected previous year (without MR) during a survey on file led on the hospital.

Main outcome measures: The aim of our study is to assess the quality of this activity organization as well as its relevance for inpatient security.

Results: From October 2012 to January 2013, 113 patients were admitted (median age $=46$ years, sex ratio $\mathrm{M} / \mathrm{F}=0.85$ ). MR could be conducted for $95 \%$, including $2 / 3$ through 48 h. $31.9 \mathrm{~min}$ are required on average per patient. An average of 2.7 information sources is crossed by patient ( $56 \%$ written, $43 \%$ oral). A patient interview is carried out in $47 \%: 2 / 3$ appears to be useful and $9 / 10$ to be easy. Physicians ensured validation of $59 \%$ of MR forms and informed the reasons for medication modifications for $28 \%$ of them. 14 unintentional discrepancies (medication error) were intercepted, relating to $10.3 \%$ of patients. It is lower than literature comparison, but our patient population is different (younger, less drugs). Nevertheless, we show that only $63.5 \%$ of investigations were ended (at least 2 corroborating sources for each line of treatment).

Survey on file led on 39 patients, (median age $=57.9$ years, sex ratio $\mathrm{M} / \mathrm{F}=0.69$ ), showed that number of sources crossed by patient was 1.41 , so reliability is increased. Written records about medication taken at home before admission appeared in $82 \%$ of patient files (versus $95 \%$ ) and about medication modifications at admission in $15 \%$ (versus $28 \%$ ).
Conclusions: In conclusion, this study showed the interest of MR for patient safety and that we can optimize some organization points to increase efficiency.

Disclosure of Interest: None Declared.

\section{HP-PC87}

Standardizing pharmaceutical messages: a key point to prescription safety improvement

C. Richard ${ }^{1, *}$, B. Berdougo ${ }^{1}$, C. Cordonnier-Jourdin ${ }^{1}$, A. Astier ${ }^{1}$, M. Paul ${ }^{1}$

${ }^{1}$ Pharmacie, GHU Henri Mondor, Assistance Publique des Hôpitaux de Paris, Créteil, France

Background and Objective: In France, health and regulation authorities urge for a constant quality improvement of the prescription and its execution. The pharmaceutical analysis of prescription may lead to the identification of pharmacotherapeutic problems synthesized in a "pharmaceutical intervention" (PI). At the hospital, most of the prescriptions as well as their analysis are computerized (Actipidos-Phedra) and PI are transmitted online to the prescribers.

The objectives of the study were: to design a PI classification to standardize the pharmaceutical message; then to identify recurrent prescription problems in order to improve the prescription upstream to the pharmaceutical validation.

Setting and Method: A PI retrospective analysis was realized in the geriatric department over 6 months (Nov. 2012 to Apr. 2013). First PI categories were selected and adapted based on the list defined by the French Clinical Pharmacy Society (SFPC). PI extracted were then sorted by these categories and analysed.

Results: 2,350 prescriptions were validated (mean of 9.6 lines by prescription) and 643 PI were made. 11 categories were selected: dosage (adjustment, usual dosage, maximal dosage, administration rate), therapeutic strategy, unreferenced drugs, contraindication, interactions, adverse reactions, biological follow-up, galenic adaptation, redundancy, errors (unit, route of administration), information. $24 \%$ of the PI concern biological follow-up whether $20 \%$ are relative to information exchanged between the physician and the pharmacist. $14 \%$ of the PI highlight prescription of non referenced drugs. Dosage problem appears in $13.4 \%$ : $43 \%$ concern administration rate problems (antihypertensives) and $41 \%$ focus on drug to be adjusted (anti-infectives and renal impairment status). Drug interactions are described in $6.2 \%$ of the cases. PI relative to errors of dosage unit or administration route, to tablet division misuses were respectively of $4.2 \%$ and $3.4 \%$.

Conclusions: Standardizing PI will enhance their relevance and legibility for prescribers and pharmacist. Revising informatics drug configuration would avoid galenic adaptation and errors problems. The edition of a leaflet containing strategies on antihypertensive rhythm of use, drug adjustment rules under renal insufficiency and therapeutic equivalents of non referenced drug may help prescribers as well. The communication between health actors is essential for a good patient coverage. The PI is a key point to its management by enforcing the safety of the prescription.

Disclosure of Interest: None Declared.

\section{HP-PC88}

Severe iatrogenic hyperkaliemia in diabetic patient: a case-report V. Ferreira ${ }^{1, *}$, E. Gerardin ${ }^{1}$, D. Zanife ${ }^{1}$, F. Vernet $^{2}$, P. Piquet ${ }^{2}$, P. Pisano ${ }^{1}$, S. Honoré ${ }^{1}$ 
${ }^{1}$ Service Pharmacie, Secteur Pharmacie Clinique, ${ }^{2}$ Service Chirurgie Vasculaire, Hôpital de la Timone, Marseille, France

Background and Objective: Hyperkaliemia is a common electrolyte disorder that often occurs in hospitalized patients and may have serious consequences which can be life-threatening.

The purpose of this clinical case description is to bring out the hyperkaliemia risk factors in order to understand how an ordinary hospitalization could lead to life-threatening.

Setting and Method: Case-report: A 63-years-old woman, with type II diabetes and high-blood pressure was admitted in a chirurgical vascular unit to follow-up surgical management of a left amputated limb ischemia.Her treatment was, inter alia, calcium heparin, insulin and candesartan as well as an antibiotic therapy with intravenous ceftazidim and oral ceftriaxone. On the first biological results, a severe hypokalemia $(2.8 \mathrm{mmol} / \mathrm{l})$ was highlighted and treated by IV and oral potassium salts. At day 3, the patient had a hyperkalemia episode $(5.2 \mathrm{mmol} / \mathrm{l})$ normalized by the immediate discontinuation of the IV potassium. On day 9, a trimethoprim-sulfamethoxazole therapy is decided in absence of clinical improvement; in parallel with insulin doses reduction which was continued over several days. At day 16, following a severe hyperkalemia $(8.5 \mathrm{mmol} / \mathrm{l})$ the patient was transferred to intensive care and initially treated with three doses of sodium polystyrene sulfonate, ineffective, then with hemodialysis. The patient gradually recovered until her exit, at day 23 .

Results: In this case, several arguments could have predicted the high risk of hyperkaliemia. During hospitalization, the candesartan dose (hyperkaliemiant) was unchanged whereas calcium heparin dose (hyperkaliemiant) was increased and insulin dose (hypokaliemiant) was decreased. The introduction of trimethoprim-sulfamethoxazol, which is known to be a powerfull hyperkaliemiant drug, while maintaining oral potassium chloride, is probably responsible of the high hyperkaliemia. Yet, according to the ANSM "Thesaurus" (French official drug's database) this association is only classified as "take into account" (4th level of four). So, a new drug interaction level seems to be necessary in view of the extensive literature about thrimetoprim-sulfamethoxazol hyperkaliemiant effect, and of this clinical case, particularly in diabetic patients. Furthermore, in these circumstances, close monitoring of potassium serum was necessary but in this case a six days delay between two biological tests has prevented the earlier detection of kaliemia increase.

Conclusions: Although Physicians and Pharmacists work together in reducing the iatrogenic risk, we note that extremely serious hyperkaliemia may occur. Thus, it seems necessary to take into account patient's physiopathology and drug interactions level in the analysis of e-prescriptions. After all, knowledge and experience sharing between Physicians and Clinical Pharmacists could ensure, for the best, patient safety.

Disclosure of Interest: None Declared.

\section{HP-PC89}

Hyperthyroidism by Nilotinib in Philadelphia ChromosomePositive Chronic Myeloid Leukemia: A case report

M. Álvarez-Payero ${ }^{1}$, C. Loureiro-Santamaría ${ }^{2}$, A. Martín-Vila ${ }^{1}$, N. Martínez-López de Castro ${ }^{1}$, M. Suarez-Santamaría ${ }^{1, *}$, G. Piñeiro-Corrales ${ }^{1}$

${ }^{1}$ Pharmacy, ${ }^{2}$ Hematology Department, University Hospital of Vigo, Vigo, Spain

Background and Objective: Thyroid dysfunction, mostly hypothyroidism, is a well-known adverse event of first-generation Tyrosinekinase inhibitors (TKI), although the mechanism is still unclear. However, hyperthyroidism in second-generation TKI, like nilotinib, is less documented. Nilotinib is indicated for the treatment of adult patients with newly diagnosed Philadelphia Chromosome-Positive Chronic Myeloid Leukemia (PP-CML) in the chronic phase. Main undesirable events: haematological toxicities, elevated bilirubin and hepatic transaminases, nausea, headache.

We describe a case of hyperthyroidism by nilotinib treatment, in PP-CML patient.

Setting and Method: Desing: Case report, evaluation and discussion based in clinical chart and literature review. Setting: Hematology and Pharmacy departments, General Hospital.

Main outcome measures: Thyroid function (thyrotropin (TSH), free thyroxine (T4), free triiodothyronine (T3); determined by immunochemiluminescence assay.

Results: A 47 year-old men presenting hyperthyroidism symptoms 15 weeks after starting nilotinib (400 mg two-daily) for PP-CML (diagnosis of his disease: 5 years ago). Previously, he was treated with imatinib $400 \mathrm{mg}$ one daily, for 4.5 years with good response, but it was stopped because of intolerance. He had not previous history of thyroid dysfunction. He did not receive medication known to interfere with thyroid function (TF). Baseline TF-test were on range: $\mathrm{TSH}=0.82 \mu \mathrm{UI} / \mathrm{mL}$ $(0.3-4.7), \mathrm{T} 4=1.16 \mathrm{ng} / \mathrm{dL}(0.89-1.76), \mathrm{T} 3=2.93 \mathrm{pg} / \mathrm{mL}(2.3-4.3)$. His TF was abnormal during nilotinib therapy, TF-test 4 months after starting nilotinib: $\mathrm{TSH}<0.02 \mu \mathrm{UI} / \mathrm{mL} \quad(0.3-4.7), \quad \mathrm{T} 4 \quad 3.14 \mathrm{ng} / \mathrm{dL}$ (0.89-1.76), T3 $8.63 \mathrm{pg} / \mathrm{mL}(2.3-4.3)$. He received antithyroid treatment with methimazole (5 mg one daily) and Nilotinib dosing was reduced from $400 \mathrm{mg}$ two-daily to $200 \mathrm{mg}$ two-daily. TSH values continued low $(<0.02 \mu \mathrm{UI} / \mathrm{mL})$. Nilotinib and methimazole was stopped 20 weeks after starting treatment, because of intolerance to nilotinib (hyperthyroidism, fatigue, arthralgia, disabling pain in extremity, gout). Six weeks after stopping nilotinib, all complaints had disappeared and the patient remained euthyroid. Adverse event by nilotinib was notified to Spanish's Pharmacovigilance Centre. Actually, he is being treated with dasatinib. Conclusions: We attribute this adverse event to nilotinib, because thyroid hormones regained normal levels after stopping treatment. In this case, hyperthyroidism by nilotinib was detected before than in other ITK, according to literature). Although hyperthyroidism by nilotinib is an uncommon adverse event, baseline TF-test and regular monitoring of TF should be performed in all patients.

Disclosure of Interest: None Declared.

\section{HP-PC91}

Impact of use of urine test strip in the prescription of cytobacteriological examination of urine

J. Daupin ${ }^{1, *}$, V. Gauthier ${ }^{2}$, F. Labouree ${ }^{2}$, A. Chevallier ${ }^{1}$, F.X. Chedhomme ${ }^{1}$

${ }^{1}$ Pharmacy Department, ${ }^{2}$ Geriatric Medicine Department, Broca Geriatric Hospital, Central Paris Hospital Group, Paris, France

Background and Objective: The cytobacteriological examination of the urine (CBEU) is the most common prescribed laboratory test in our geriatric hospital. A preliminary test by urinalysis strips (US), easier to use and cheaper ( 0.17 vs $17 €)$, has an excellent negative predictive value and allows to limit unnecessary tests. The aim of this study is to improve the professional practices by performing a focused clinical audit, setting up locally adapted recommendations and evaluating their impact on relevance of prescriptions.

Setting and Method: Program of professional practices evaluation in 3 phases: 1/Prospective study of CBEU during 3 months with a questionnaire drawn up by a multidisciplinary group (pharmacists, doctors and bacteriologists) 2/Implementation of improvement actions plan included education for physicians and a warning note in the hospital prescription software concerning the new recommendations (systematic use of US before the CBEU and traceability of the 
US result in patients medical records) 3/Prospective analysis of CBEU prescriptions 3 months after.

Main outcome measures: Relevance of the request of biological examination after medical records review (clinical signs, US results); adherence to recommendations (preliminary result of US, traceability of US results).

Results: 107 CBEU were analyzed in the first phase and 100 in the third. The CBEU done without US decreased from $71(66 \%)$ to $39(38 \%)$. However the number of CBEU made in spite of a negative US stayed quasi similar between the two phases (4 vs 5). The number of ECBU prescribed with a clinical justified reason raised from $73(68 \%)$ to 95 (95\%). The principal clinical causes were fever $(52 \%)$, pelvic pain (12\%), the appearance (4\%) and the smell (2\%) of the urine. However the traceability of US results in medical records did not significantly improve $(2.5 \%)$.

Conclusions: The good use of the CBEU improved after implementation and education in prescribing guidelines. From now the systematic use of US allows to rationalize the CBEU prescriptions. As with medication, clinical pharmacy can improve the effectiveness of laboratory tests prescriptions and impact the quality of care and the costs. This study of an educative assessment program shows the interest to make a current status evaluation of practices before proposing focused and relevant corrective measures.

Disclosure of Interest: None Declared.

\section{HP-PC92}

Rationalisation of carbapenems consumption: Evaluation of the impact of controlling the prescription in the Hospital Santo André of Centro Hospitalar Leiria-Pombal.

\section{A. I. Rodrigues ${ }^{1, *}$, A. M. Bravo ${ }^{1}$, A. F. Fernandes ${ }^{1}$, R. C. Marques ${ }^{1}$}

${ }^{1}$ Pharmacy department, Centro Hospitalar Leiria-Pombal, E.P.E., Leiria, Portugal

Background and Objective: Carbapenems are a group of broad spectrum antibiotics and therefore should only be prescribed after a careful clinical evaluation, leading to a rationalized use of these drugs. Its indiscriminate or inadequate use might result in the development of antibiotic resistance which will consequently lead us to a serious public health problem.

In 2007 after having confirmed an elevated consumption of this group of antibiotics, the hospital's clinical council established the requirement for superior validation of these prescriptions, by the clinical director(CD).

This study aims to evaluate the impact of superior validation in the prescription profile of these antibiotics.

Setting and Method: Retrospective study of the carbapenems consumption, in defined daily doses (DDD), in the hospital from 2002-2012. Statistical analysis of the results.

Main outcome measures: Collection of consumed carbapenem vials (Meropenem and Imipenem + Cilastatin) (N) in 2002-2012 period. Calculation of DDD (WHO criteria) and DDD/1,000 patients discharged from the hospital (I).

Results: The consumption results in N/DDD/I were: -2002-9815/ 3918/162,0-2003-12075/3569/187,7;-2004-10030/3082/164,5;-200510924/3303/179, 7 ;-2006-8743/2609/135,8;-2007-6241/1911/99,4; 2008-5042/2143/104,1;-2009-6453/3227/158,1;-2010-7158/3579/ $\overline{174,4} ;-\mathbf{2 0 1 1}-6257 / 3129 / \overline{150,9} ;-\mathbf{2 0 1 2}-5402 / 2701 / \overline{128,3}$.

The decision to rationalize the use of carbapenems in the hospital resulted in an immediate $30 \%$ decrease in 2007. A slight $12 \%$ increase was verified in 2008 , without significance.

The collected data revealed another increase in 2010, which may be explained by an outbreak of Acinectobacter baumannii in the hospital, with 188 identified cases.
Since 2011 there is a trend towards reduction.

Conclusions: The rationalization measures had a noticeable impact in carbapenems use throughout the process, although with more significance in the first year. The initial reaction was the non-prescription. Nevertheless, subsequently, the habit was resumed more rationally.

The consumption trend observed shows that the pharmaceutical validation prior to the implementation of this measure was based on strict criteria, given that consumption levels are now reaching the values observed before.

In the long term this measure had a fundamental role in causing awareness of the bacterial resistance problem, being landmark in rationalizing their consumption of carbapenems.

The data analysis shows a trend towards reduction since 2011, which reflects a more rational prescription. Ultimately this leads to minimizing the development of antibiotic resistance.

Disclosure of Interest: None Declared.

\section{HP-PC93}

Drug flow system in pediatric intensive care unit (ICU): inventory in French teaching hospitals

\section{J. Thiec ${ }^{1, *}$, K. Beuzit ${ }^{1}$, D. Gueylard-Chenevier ${ }^{2}$ \\ ${ }^{1} \mathrm{CHU}$ de Poitiers, Poitiers, ${ }^{2} \mathrm{CHIP}$ de Cognac, Cognac, France}

Background and Objective: Following many errors in pediatric ICU, a multidisciplinary feedback committee was established. It was then decided to have an audit on practices concerning the drug flow system in order to identify improvement tracks and discuss tools used between the various institutions.

Setting and Method: A survey including 15 items built from different official medical and pharmaceutical guides ("Tools to improve security and self evaluation in drug administration", "administration in pediatrics") and French regulation texts (2011, April 6th) was sent to 32 hospital pharmacies, to be filled out by a pharmacist.

Themes addressed: drug prescription, pharmaceutical performances, administration, drug error monitoring system.

Results: Drug prescription is computerized in 1 establishment out of every 2 .

Oral prescription is standard practice in $12 \%$ of cases.

Analysis of the prescriptions is complete in only $57 \%$ of the establishments, but the rate reaches $71 \%$ when referring to "risky drugs".

Regarding drug administration, an adapted preparation is often required. The dose calculation is based on a written support (86\%), it is included in the patient file (21\%) and nurses can use conversion tables $(29 \%)$. Nurses can be double-checked by a colleague automatically (29\%), if a risky drug is used (29\%), if the drug is not familiar $(29 \%)$ or if there was a dose calculation $(14 \%)$.

These preparations are labelled with the patient's identity (86\%), the drug's name, dose and dilution $(79 \%)$, and time of preparation $(57 \%)$.

The administration is generally correctly registered.

Finally, only half of the hospitals surveyed have developed a drug error monitoring system.

Conclusions: This survey shows that implementation of regulations remains difficult. However, prescription analysis seems to be increasing.

Some good practices can be noted:

- a specific quiet area dedicated to drugs' preparation

- pre-diluted syringes prepared within the pharmacy

- preparation's label: add rate of flow, date, nurse's identity

- $\quad$ specific training for pediatric caregivers. 
Even if having a drug error monitoring system is now an obligation, only a few hospitals seem to apply it today.

Finally, we can only regret that this survey did not allow to evaluate team work, for a large number of errors are due to lack of communication. Disclosure of Interest: None Declared.

\section{HP-PC94}

Prospective study of medication reconciliation in postoperative patients undergoing orthopaedic surgery.

B.-G. Margarita ${ }^{1, *}$, C.-A. Ana ${ }^{2}$, F.-M. Mónica ${ }^{3}$, F.-A. Juan-Antonio ${ }^{4}$, M.-M. Marisa ${ }^{1}$, G.-A. M $^{\mathrm{a}}$ Dolores $^{1}$

${ }^{1}$ Farmacia, ${ }^{2}$ Ortopedia y Traumatología, Hospital Universitario Virgen Macarena, Sevilla, ${ }^{3}$ Farmacia, Hospital Universitario Virgen de las Nieves, Granada, ${ }^{4}$ Facultad de Farmacia, Universidad de Sevilla, Sevilla, Spain

Background and Objective: The postoperative reconciliation is a complex process where high risk reconciliation errors can occur, but according to studies the incidence is very diverse.

To analyze the impact of pharmaceutical interventions in avoiding potential reconciliation errors in orthopaedic surgery patients and for $48 \mathrm{~h}$ postoperative.

Setting and Method: A prospective and observational study in March 2012

We selected patients over 60 years, orthopaedic surgery and with $\geq 1$ chronic medications.

The pharmacist, made the following tasks:

1. Collect information on home treatment of the patient.

2. Confirm the information by interviewing the hospitalized patient.

3. pharmaceutical intervention, either update the patient's prescription profile or verbally recommend clinical action regarding drugs.

This was supplemented with clinical validation of pharmaceutical intervention in less than $24 \mathrm{~h}$.

Main outcome measures: The intervention method was: $91.6 \%$ update the prescription profile and $8.3 \%$ a clinical recommendation. Acceptance of interventions: $97.23 \%$.

Results: Of the patients reviewed, $38 \%(\mathrm{n}=188)$ were selected. There were $66 \%$ women, mean age 73 years, $74 \%$ with $\geq 3$ comorbidities, and an average of 6 medications. $72 \%$ of patients brought drugs on admission, but only $50 \%$ admitted their intention to take it.

We detected a total of 180 unjustified discrepancies in the 414 chronic medications $(43.5 \%)$ and $87.32 \%$ of affected patients.

The distribution of the type of unjustified discrepancies: omission: 127 (70.5 \%); not stopping the drug: 28 (15.5\%); and duplicity: $25(13.8 \%)$.

In relation to gravity, the error: $75 \%$ would require monitoring of medication to prevent damage $22 \%$ would not have caused damage and $2.8 \%$ had caused damage,

Conclusions: $87 \%$ of patients had an error of reconciliation, $75 \%$ of errors require medication monitoring to prevent harm and the intervention was accepted by $97 \%$, reflecting the importance of the integration of reconciliation in the pharmacist's daily activity.

Disclosure of Interest: None Declared.

\section{HP-PC95}

Pediatric preparations made in the unit versus in the central pharmacy

\author{
A. Adamo' ${ }^{1}$, P. Polidori, ${ }^{1 *}$ \\ ${ }^{1}$ Clinical Pharmacy, ISMETT, Palermo, Italy
}

Background and Objective: Pediatric therapies are usually characterized by the off label use of adult drugs with adjustment of the dosage because of the lack of appropriate formulations. The Clinical Pharmacy Service includes a unit dose drugs compounding section to personalize therapies according to the real patient needs. Furthermore this system allows preparing many doses from a vial according to dosage and drug stability.

Object of the study is to analyze the cost of pediatric centralized formulations compared to drug preparations made by nurses in their units.

Setting and Method: A prospective observational study was carried out in a transplant hospital where pediatric drug preparations are centralized in the Pharmacy.

Main outcome measures: From May to August 2012 extemporaneous pediatric unit doses report was analyzed. The cost of each dose was given by the sum of cost of drugs, medical and individual protection devices and personnel, according the time spent by pharmacists and technicians for the management of the preparations. The same evaluation was done for drugs hypothetically prepared by nurses and it was compared with the cost of centralized compounding. Results: In the analysis period 2084 pediatric extemporaneous preparations were made, which corresponds to $6.1 \%$ of all compounded formulations developed. The unit doses were for $21.3 \%$ oral formulas and for $78.7 \%$ sterile parenteral preparations. The most common drugs were diuretics $(28.8 \%)$, systemic antibiotics $(26.7 \%)$, mineral integrators $(14.8 \%)$, immunosuppressants $(12.7 \%)$, proton pump inhibitors $(12.2 \%)$ and others $(4.8 \%)$.

The cost of all doses was $27,420.01 €$. To the management of the preparation process the pharmacists spent $44 \mathrm{~h}$ and the technicians $35.7 \mathrm{~h}$ with a cost respectively of $928.40 €$ and $581.58 €$. So the total cost of the process was $28,929.99 €$.

The hypothetic cost of the preparations made by nurses was $34,495.15 €$, and plus nurses cost of $581.58 €$, the total cost became $35,076.73 €$.

So the centralized preparation allowed saving 6,146.74€ in 4 months. In a year it could be possible to save about $18,400 €$.

Conclusions: The centralized preparation allows improving the quality and safety of the treatment, reducing the variability in prescriptions through the dosage standardization and, thanks to the optimization of hospital resources, to obtain financial benefit.

Disclosure of Interest: None Declared.

\section{HP-PC96}

Use of omalizumab in the treatment of refractory chronic urticaria

M. Simon,", E. Dahan ${ }^{1}$, M. P. Ponrouch ${ }^{1}$, I. Roch-Torreilles ${ }^{1}$, P. Rambourg ${ }^{1}$

${ }^{1}$ Pharmacy Saint Eloi-Gui de Chauliac, CHRU Montpellier, Montpellier, France

Background and Objective: Chronic urticaria is defined by the persistence of skin lesions beyond 6 weeks. H1-antihistamines represent the conventional treatment. Omalizumab is a monoclonal antibody approved for the treatment of allergic asthma. Several case report seem to demonstrate its effectiveness in chronic urticaria.

Setting and Method: The study took place at the Teaching Hospital of Montpellier.

Main outcome measures: Our work reports three cases of patients with chronic urticaria refractory to recommended treatment and treated by omalizumab.

Results: The first patient of 67 years has cold urticaria since 20 years. Omalizumab was initiated at a dose of $375 \mathrm{mg}$ every 2 weeks. From the first injection, there was an improvement in symptoms with a 
decreased incidence and severity of crises (one single episode between the first and third treatment despite regular exposure to cold and fresh water). Given the good response, injections were spaced out 3 then 4 weeks with good results (negative ice cube test, the patient may bathe and eat ice cream) and good tolerance (no infection and no complications post injection).

Given the effectiveness of this therapy, two other patients with chronic urticaria treatment failure received Omalizumab.

The second patient ( 54 years old) has heat urticaria since one year with multiple daily attacks. The patient received omalizumab, $300 \mathrm{mg}$ every 15 days. After the first injection, there is an improvement of the symptoms. Some crises are then reported, but less intense than before. There are no adverse effects of the treatment.

The third patient of 23 years old has chronic urticaria evolving since 2003. Omalizumab was introduced at a dose of $300 \mathrm{mg}$ every 15 days. Given a partial improvement (decrease of the intensity of persistent daily crisis), the doses was increased: $375 \mathrm{mg}$ every 15 days. The expected benefit is being studied.

Conclusions: Omalizumab appears to be an effective and well tolerated alternative therapeutics in case of chronic urticaria refractory to conventional treatment. The absence of AMM and the important cost of this drug is a limit to its use. Omalizumab should be initiated case by case after multidisciplinary consultation. Clinical development with randomized studies on a large cohort of patients is needed to prove the efficacy and safety of omalizumab in this indication.

Disclosure of Interest: None Declared.

\section{HP-PC97}

Drug safety data of Regorafenib in metastatic colorectal cancer

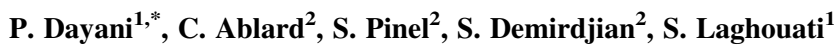
${ }^{1}$ Pharmacovigilance Unit, ${ }^{2}$ Clinical Pharmacy Department, Gustave Roussy, Villejuif, France

Background and Objective: This study was performed in order to monitor the safety data of Regorafenib in patients being treated in Gustave Roussy Institute.

Setting and Method: Drug safety data have been collected prospectively during 3 months, for all treated patients with Regorafenib, a multi-kinase inhibitor, in a context of a Temporary Authorization for Use (TUA), based on their medical records and data entered by the prescribing physicians. The initial dose was $160 \mathrm{mg}$, orally once daily for the first 21 days of each 28-day cycle.

Results: Eight patients have been treated. The median age was 51 years old [40-74] and sex ratio M/F 0.6. $50 \%$ of patients carried KRAS mutation. $87.5 \%$ of patients received at least 3 previous lines of chemotherapy before Regorafenib and had at least 3 metastasis locations (mainly hepatic and lung). The median duration of treatment was 55 days [21-117].

$75 \%$ of patients experienced at least one Adverse Drug Reaction (ADR), for a total of 31 ADRs. $75 \%$ of ADRs were grade I or II. Two ADRs were serious (requiring patient's hospitalization): a grade 3 CTCAE pancreatitis (also possibly related to a vesicular lithiasis) and a grade 2 dyspnea. Three patients experienced skin toxicity including palmar-plantar erythrodysesthesia with a positive re-challenge for one of them. Other frequently observed ADRs were $13 \%$ gastrointestinal (diarrhea, gingivitis, mucositis) and $27 \%$ hepatic disorders (transaminases, AKP, GGT, bilirubin increase). However, the presence of liver metastases in these patients makes it difficult to assess causality.

These ADRs induced a temporary treatment discontinuation for 3 patients. Four $(50 \%)$ patients discontinued treatment due to disease progression and 2 patients $(25 \%)$ died during treatment related to tumor progression. Two patients are still treated.

Conclusions: Regorafenib was well tolerated. ADRs' nature and frequency in our cohort are similar to those observed in clinical trials. This should be confirmed on a larger number of patients and a longer exposition once the drug will be marketed. Regorafenib is administered as 3rd line therapy, which explains a high rate of disease progression and death during treatment. Three new TUAs have been requested since.

Disclosure of Interest: None Declared.

\section{HP-PC98}

Complexity of pluripathological inpatients's pharmacological treatment in a Internal Medicine service

E. Fernandez-Gabriel, ${ }^{1, *}$, M. Mauriz-Montero ${ }^{1}$, M. Rabuñal-Alvarez ${ }^{1}$, C. Vilaboa-Pedrosa ${ }^{1}$, M. Mateos-Salvador ${ }^{1}$, M. Martin-Herranz ${ }^{1}$

${ }^{1}$ Servicio De Farmacia, Complexo Hospitalario Universitario A Coruña, A Coruña, Spain

Background and Objective: Ageing population and improvements in diagnosis, therapeutic approach and treatment of illness are increasing the number of pluripathological patients (PPP) in whom coexist several chronic diseases. The aim of this study is to describe the $\mathrm{PPP}^{\prime} \mathrm{s}$ treatment complexity and how this is affected by the hospitalization.

Setting and Method: Retrospective observational study. Period: January 2013. Sample: $100 \%$ hospital discharges in 3 units of internal medicine in a tertiary hospital ( 1,419 beds). Inclusion criteria: PPP, patients with at least 2 clinical categories at discharge, based on Ollero $\mathrm{M}$ et al. classification. Data collected: demographics, length stay hospital, diagnosis at admission, chronic diseases at discharge, type of drugs, number of drugs and doses/patient/day at admission and discharge. Data extraction: electronic clinical history (IANUS ${ }^{\circledR}$ ). Pharmacoherapeutic profile at admission was compared with pharmacotherapeutic profile at discharge.

Results: 104 patients were included (57.7\% male), median age 76 years $(84 \%>65$ years, $35 \%>85$ years). Average length stay: 8.4 days. Admission causes: $32 \%$ respiratory, $24.4 \%$ cardiac. Main clinical categories: $54.8 \%$ category A (36 patients-cardiac insufficiency, 26-ischemic cardiopathy), $42.3 \%$ category C (43-chronic respiratory disease), $38.5 \%$ category $\mathrm{B}$ (12-vasculitis or systemic autoimmune diseases, 31-chronic renal disease), $37.5 \%$ category $\mathrm{F}$ (10- peripheral arteriopathy, 33-diabetes mellitus associated with proliferative retinopathy or symptomatic neuropathy). At admission: $8.9 \mathrm{drugs} / \mathrm{patient} /$ day and $11.6 \mathrm{doses} /$ patient/day. At discharge: 10.1 drugs/patient/day and 13.3 doses/patient/day. 56/104 patients at least 1 high-risk medication and 26/104 at least 2 high-risk medications (acenocoumarol, insuline, oral antidiabetic drugs, opioides and cytostatic drugs). Chronic treatment was modified after hospital admission: $48 \%$ addition of new drugs, $29.8 \%$ deletion of drug from therapy, $25 \%$ dosage adjustment and $13.6 \%$ change of active principle. In $50 \%$ of PPP finalization of treatment at discharge (antibiotics, low molecular weight heparin, oral corticoids and diuretics).

Conclusions: It particularly highlights the complexity of $\mathrm{PPP}^{\prime} \mathrm{s}$ pharmacological treatment. This complexity increases after hospitalization and requires ongoing support shared between primary and specialized care. Pharmacist is a basic pillar in interlevels care reconciliation, and requires extensive training in the major diseases affecting these patients.

Disclosure of Interest: None Declared. 


\section{HP-PC99}

Development of clinical pharmacy intervention (PI): a six weeks evaluation in a french internal medicine unit.

Y. Audurier ${ }^{1}$, R. Stehle ${ }^{1}$, M. Villiet ${ }^{2, *}$, C. Breuker ${ }^{2}$, J. Ribstein ${ }^{1}$, S. Hansel-Esteller ${ }^{2}$

${ }^{1}$ Internal Medicine, ${ }^{2}$ Pharmacy Lapeyronie, CHRU Montpellier, Montpellier, France

Background and Objective: Internal medicine patients often old and polymedicated, are at risk of adverse drug events. Clinical pharmacy practice may reduce medications errors. The implementation of clinical pharmacy activities with the medical and paramedical teams may improve the management of these patients. We assess quantity and type of pharmacist intervention (PI) by one pharmacist resident related to patient's medication management.

Setting and Method: We conduct a prospective study during six weeks in medicine department. The resident analyse all prescriptions, except during week-end. PI are realized before transmission of the prescription to the pharmacy unit.

Main outcome measures: Quantity and type of PI, drug class are recorded. Only the accepted PI are recorded. PI concern addition or suppression of therapy, pharmacologic interactions, substitution, optimization of anti-infectives, dosage regimen, biological alert, optimized administration, nurses' pharmacological assistance, physicians' pharmacological assistance and help/correction of new electronic prescription.

Results: 542 interventions have been registered on a population of 105 patients. The patients mean age is approximately 71 years old. The PI concern 174 (32.1\%) treatment optimization (galenic, dosage or biological controls), 171 related to recent computerization $(31.5 \%)$, $102(17.5 \%)$ assistances of nurses and physicians and $95(17.5 \%)$ concern treatment choice (addition, suppression or substitution). Main therapeutic classes are: Anticoagulants $(19.3 \%)$, Antibiotics (19.3\%), Antalgics (8.0\%), Cardio-vascular drugs (12.5\%), oral antidiabetics $(9.1 \%)$, benzodiazepines $(8.0 \%)$, antihypertensive drugs $(14.8 \%)$, Antidepressants drugs $(5.7 \%)$ and other drugs $(10.8 \%)$.

Conclusions: Approximately 18 PI by day are effected by one pharmacist resident integrated in a medicine unit showing the clinical pharmacy requirement. Integrated pharmacist improves drug manipulation, protocol adherence, optimized dosage, treatment cost, similarly to other study and shows a great impact in deployment of computerized prescription. PI realized directly with physicians or nurses reduced times to correction or information and so, improve patient's medical management. However, the clinical impact of PI is needed, and must be evaluated in a second time.

Disclosure of Interest: None Declared.

\section{HP-PC101}

Pharmaceutical interventions in patients with domiciliary enteral nutrition

C. Vázquez-López ${ }^{1}$, A. Martín-Vila ${ }^{1}$, M. Álvarez-Payero', M. Suárez-Santamaría ${ }^{1, *}$, N. Martinez-López de Castro ${ }^{1}$, G. Piñeiro-Corrales ${ }^{1}$

${ }^{1}$ Pharmacy, University Hospital of Vigo, Vigo, Spain

Background and Objective: Patients on Domiciliary Enteral Nutrition with feeding tube (DENFT) (percutaneous endoscopic gastrostomy (PEG) nasogastric feeding tubes (NFT) and jejunal feeding (JF)) frequently presents Problems Related to Enteral
Nutrition (PREN) Our purposes were to describe the type of patients with DENFT, to analize the (PREN), and the pharmaceutical interventions.

Setting and Method: Descriptive and prospective study in an University hospital for 12 months. All patients with DENFT were monitorized by a pharmacist integrated in Nutricional Support Team (NST).

Main outcome measures: Data collected: age, sex, type of patient (community care or nursing home), principal diagnosis, enteral nutrition type, exitus during study period, type of PREN: Indication(I), Efficacy(E), Safety(S) and pharmaceutical intervention (PI) (nutritional assessment based in: clinical interview of the patient, MNA: Mini Nutritional Assessment, and biochemical parameters).

Results: 184 patients $(62.5 \%$ women $)$ were included, mean age: $79.2 \pm 14.4$ years. $70.6 \%$ were community care and $29.3 \%$ were nursing home. $52.2 \%$ of patients had PEG, $46.7 \%$ NFT and $1.1 \%$ JF. Main diagnosis were: dementia (42.9\%), cerebrovascular accident $(32.1 \%)$, malnutrition $(14.1 \%) .38, \%$ of patients received standard diet, $28.3 \%$ fiber, $10.3 \%$ combination of standard and fiber, $8.7 \%$ diabetic, $5.4 \%$ high-protein and $9.3 \%$ others enteral nutrition types. $27.6 \%$ patients were exitus, during study period. 78 PRNE were detected (0.4 ENRP/patient). Type of ENRP: $56.4 \%$ E, $28.2 \%$ I, $15.4 \%$ S. $94 \%$ pharmaceutical interventions were change nutritional support, $3.8 \%$ start nutritional support and $1 \%$ stop nutritional support Conclusions: Most patients are over 75 years and have PEG. The PREN more frequent were relationated with Efficacy. A high number of pharmacist interventions were performed in patients with DNFT. The farmacist integrated in NST decreases risk of complications in patients with DENFT.

Disclosure of Interest: None Declared.

\section{HP-PC103}

Physician-pharmacist collaborative intervention in the drug therapy optimization in patients with type 2 diabetes

P. M. Aguiar ${ }^{1}$, E. L. Dórea², G. A. Pinto², M. C. Gusukuma², D. P. Lyra Jr' ${ }^{3}$, S. Storpirtis,

${ }^{1}$ Faculty of Pharmaceutical Sciences, University of São Paulo, ${ }^{2}$ Metabolic Diseases Clinic, University Hospital of São Paulo, São Paulo, ${ }^{3}$ College of Pharmacy, Federal University of Sergipe, São Cristóvão, Brazil

Background and Objective: Drug therapy problems (DTPs) constitute a major public health problem because of their consequences on morbidity, mortality and cost. Involving pharmacists in type 2 diabetes multidisciplinary care teams may enhance patient education and improve medication use. The purpose of this study was to evaluate the type of pharmacist's interventions and the acceptance of these interventions by physician to resolve DTPs.

Setting and Method: A 10-month prospective study of medication management was conducted at Metabolic Diseases Clinic, University Hospital of São Paulo, Brazil. Patients were enrolled if they had been diagnosed with type 2 diabetes, were aged 40 to 79 years and presented glycosylated hemoglobin greater than $7 \%$. The pharmaceutical interviews were conducted face-to-face after the medical appointments, along with remote support by telephone. Pharmacists performed systematic interventions aiming to solve and prevent DTPs in the diabetic patients. Identified DTPs were put forward to the health care team and/or patients and discussed. Information on identified problems, and their outcomes was collected and analyzed. Main outcome measures: The number of DTPs identified by the clinical pharmacist; the type of pharmacist's interventions and the acceptance of these interventions by physician to resolve DTPs. 
Results: Of the 40 patients enrolled, the mean age was 61.5 years $( \pm 7.7)$, and $67.5 \%$ were female. A total of 143 DTPs were documented with the mean number of $3.6 \pm 1.9$ per patient. The DTPs identified were mainly related to effectiveness $(33.6 \%)$ and safety $(30.8 \%)$. Of all interventions to resolve DTPs, 111 (77.6\%) were negotiated and agreed upon directly between the patient and clinical pharmacist, especially about specific instructions on proper use of medication (e.g. take/use in specific time/s and relation to food). The physician was involved in the remaining interventions (22.4\%); most frequently to change the dosage regimen $(53.1 \%)$ and change the drug product $(9.4 \%)$. Almost all these recommendations were accepted by physician (96.9 \%) and $86.0 \%$ of the DTPs were solved/ prevented during patients follow-up.

Conclusions: The clinical pharmacist's interventions were mainly directed to instruct the patient on how properly use medications and there was a good intervention acceptance rate by physician. The collaborative management of treatment was critical to the resolution and prevention of DTPs.

Disclosure of Interest: None Declared.

\section{HP-PC104}

Focus on nutritional practices in a regional cancer centre after implementation of improvement actions

\section{Desbouis ${ }^{1, *}$, J. Rouvet ${ }^{1}$, I. Larivière ${ }^{2}$, S. Traversier $^{3}$,}

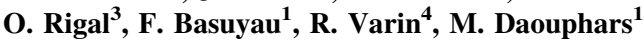

${ }^{1}$ Pharmacy, Cancer Centre Henri Becquerel, Rouen, ${ }^{2}$ Pharmacy, Centre Hospitalier de Gonesse, Gonesse, ${ }^{3}$ Supportive Care, Cancer Centre Henri Becquerel, ${ }^{4}$ Pharmacy, University hospital Charles Nicolle, Rouen, France

Background and Objective: Improving nutritional practices for hospitalized patients is paramount, especially in cancer, for which malnutrition has a major impact on morbi-mortality. After the implementation of improvement actions in our centre we performed a second evaluation of nutritional practices on the basis of a first study in 2009.

Setting and Method: A one-day prospective study, based on a clinical audit method, thanks to a multidisciplinary collaboration between medical staff, dieticians and pharmacy.

Main outcome measures: Demographic data, disease and treatment history, clinical status, biological measures and prescription related to nutrition were collected. Patients' daily oral intakes were calculated in terms of calories and proteins. Nutritional status was evaluated through Body Mass Index (BMI), weight loss and nutritional risk index (NRI). Assessment of an adequate nutritional treatment was deducted from the ratio between energy and protein requirements (evaluated by Harris-Benedict equation), and observed intakes (both food and nutritional support).

Results: Since 2009 we have established a local nutrition committee, including a nutritionist doctor helped with dieticians and referents in each functional unit. Screening tools for malnutrition and information on nutrition products have been proposed for health professionals. Among the 84 patients hospitalized in oncology and hematology (no significant difference with demographic and medical data from 2009), all screening markers indicate a lesser proportion of patients suffering from malnutrition (BMI: $14 \%$ vs. $21 \%$; weight loss: $29 \%$ vs. $40 \%$; NRI: $57 \%$ vs. $75 \%, p<0.05)$. As in 2009 , nutrition care remains globally insufficient. A nutritional support is proposed for $42 \%$ vs. $49 \%$ of patients, with less oral support ( $21 \%$ vs. $27 \%$ ), more enteral nutrition (18\% vs. $13 \%)$, and a stable parenteral nutrition (11\% vs. $12 \%$ ). Energy and protein intakes are meanwhile improved (47\% vs. $43 \%$ and $45 \%$ vs. $36 \%$ respectively). Therefore, the quality of care appears to be more effective.
Conclusions: We show a lesser proportion of undernutrition in 2013. However, nutrition care has not improved as expected. An additional study as interviews with healthcare professionals will be performed to identify other limiting factors to enhance practices.

Disclosure of Interest: None Declared.

\section{HP-PC105}

Profile of Meropenem usage in Hospital Santo André of Centro Hospitalar Leiria-Pombal,EPE

R. C. Marques ${ }^{1, *}$, A. M. Bravo ${ }^{1}$, A. F. Fernandes ${ }^{1}$, A. I. Rodrigues ${ }^{1}$

${ }^{1}$ Pharmacy department, Centro Hospitalar Leiria-Pombal, E.P.E., Leiria, Portugal

Background and Objective: Carbapenems are a group of broad spectrum antibiotics and therefore, should be prescribed in the sequence of a criterious clinical evaluation. The non-rational use of this group of antibiotics might result in the development of bacterial resistance, which is a serious public health problem.

The aim of this study is the evaluation of Meropenem prescription profile in the hospital during 2012.

Setting and Method: Retrospective study of the Meropenem use in the Hospital Santo André of Centro Hospitalar Leiria-Pombal, EPE in the year 2012. Data were collected by consulting the patient's clinical process. Descritive statistical analysis of the results.

Main outcome measures: Collected data: number of Meropenem prescriptions; number of treated patients; average age; medical specialty; infection type; type of antibiotheraphy; average dosage; average number of days of treatment (NDT); existence of previous antibiotheraphy; the identified microorganisms and the existence of co-infections.

Results: 193 prescriptions of 191 patients, average age 66.4 years; the major Meropenem prescribers in our hospital were the surgery and medicine departments, to face infections of the gastrointestinal $(28.5 \%)$ and respiratory tract $(25.9 \%)$; co-infections were present in $13 \%$ of the patients; the average dosage was $3 \mathrm{~g}$ daily for $11.4 \pm 6.9$ days; the empirical prescriptions represented $52.3 \%$ of the total; In $36.3 \%$ of the prescriptions there had been previous therapeutic with other anitibiotics; the most identified microorganism was Klebsiella Pneumoniae (23\%); for $21.4 \%$ of the total prescriptions there was no antibiogram to support the prescription; no microorganism isolation was observed in $27.8 \%$ of the cases.

Conclusions: The data collected revealed the existence of a significant percentage of empirical prescriptions. A close view of the data shows that although empiric, the prescriptions followed established protocols or were situations of previous antibiotic therapeutic failure, which reflects a more criterious and rational prescription habit.

Disclosure of Interest: None Declared.

\section{HP-PC106}

Fluindione: a complex interaction

J. Fulcrand, ${ }^{1, *}$, M. Auffret ${ }^{1}$, L. Ferret ${ }^{1}$, S. Pagnier ${ }^{2}$, J. Béné ${ }^{1}$, S. Gautier ${ }^{1}$

${ }^{1}$ Centre Régional de PharmacoVigilance de Lille, CHRU Lille, Lille, ${ }^{2}$ Polyclinique du ternois, Saint-Pol-sur-Ternoise, France

Background and Objective: Fluindione is the most commonly used oral anticoagulant drug in France, susceptible to drug-drug interactions (DDI), particularly with antibiotics. When concomitant drugs are prescribed with fluindione, more frequent monitoring of the 
International Normalized Ratio (INR) and doses adjustments are generally recommended because of thrombotic and hemorrhagic risks. We report a case of a common DDI with fluindione, leading to sequential doses adjustments.

Setting and Method: The patient was initially treated by fluindione $35 \mathrm{mg}$, for atrial fibrillation. He underwent an orthopaedic surgery and heparin treatment was initiated in relay to fluindione. He received antibiotics (cefazoline and levofloxacin) replaced one week later by levofloxacin and rifampicin, and fluindione initiated at a lower dosage $20 \mathrm{mg}$, based on infectious diseases specialist recommendations.

Main outcome measures: $48 \mathrm{~h}$ later the INR dropped sharply to 5.7. Fluindione was ceased for two days and INR value reached the therapeutic range 2.8. A dosage at $10 \mathrm{mg}$ was restarted but the next day, the INR decreased to 2 . The INR fell below the target range and stagnated to 1.4 , despite a gradually dosage increase to $35 \mathrm{mg}$.

Results: The first DDI is a pharmacodynamic interaction, resulting from vitamin $\mathrm{K}$ deficiency secondary to elimination of bacterial intestinal flora that synthesize vitamin $\mathrm{K}$. Broad spectrum antibiotic enhance, through this mechanism, the anticoagulant effect of oral anticoagulants, increasing the risk of bleeding. It is particularly described with fluoroquinolones and $\beta$-lactam, and begins within 1 to 2 weeks after initiating antibiotics. The second DDI is due to a pharmacokinetic interaction between rifampicin and fluindione. Rifampin is a potent inducer of the hepatic cytochrome P450 oxidative enzyme system, a pathway responsible for the metabolism of many drugs, such as oral anticoagulants. Induction of fluindione metabolism results in accelerated fluindione clearance, a diminished anticoagulant effect and a subtherapeutic INR. Rifampicin induction begins 5 to 7 days after rifampicin initiation and persists 5 days after it discontinuation, conducting to necessary fluindione dose adjustment.

Conclusions: In such a frequent case, a closely monitoring of INR is necessary to perform fluindione dose adjustment during antibiotic treatment and particularly because of DDI difficult to forecast.

Disclosure of Interest: None Declared.

\section{HP-PC107}

DokuPIK $^{\circledR}$ : Pharmaceutical interventions: Do ward pharmacists know how to document?

\section{A. Ihbe-Heffinger ${ }^{1,2, *}$, C. Langebrake ${ }^{2}$, K. Leichenberg ${ }^{2}$, H. Hilgarth', C. Hohmann', M. Kunkel², M. Lueb², T. Schuster ${ }^{3}$}

${ }^{1}$ Department of Gynecology and Hospital Pharmacy, Klinikum rechts der Isar der Technischen Universität München (TUM), München, ${ }^{2}$ Ausschuss Dokumentation und Evaluation pharmazeutischer Interventionen, ADKA, Berlin, ${ }^{3}$ Institute of Medical Statistics and Epidemiology, Klinikum rechts der Isar der Technischen Universität München (TUM), München, Germany

Background and Objective: To conduct a survey-based study on the clinical usability of the internet based categorical, hierarchical documentation system DokuPIK ${ }^{\circledR}$ for routinely pharmaceutical interventions (PIs) directed by hospital pharmacists.

Setting and Method: Out of 498 registered DokuPIK ${ }^{\circledR}$ users, 37 hospital pharmacists volunteered to independently evaluate 24 standardized case reports [1] between Jan/Mar 2012. Case evaluation was restricted to the reason of PI and performed based on 16 given survey items with no limit on the number of item choices. Ratings were conducted electronically and anonymously.

In order to define meaningful reference item selections, majority decisions made by 5 senior clinical pharmacists were considered as gold standard.

For purpose of sensitivity analysis, the degree of reference judges' consent for a positive vote ("item is acceptable") was altered from a majority $(60 \%)$ to a minor or maximum consensus $(20 \% ; 100 \%)$.
Main outcome measures: Agreement of raters' case evaluation with the gold standard was assessed by calculating proportion of false positive and false negative answers, sensitivity, specificity, positive (PPV) and negative predictive value (NPV) and were reported as median and range.

Results: Independent assessment yielded a median agreement of $90 \%$ [79-94\%]. False positive ratings were raw with only $1 \%$ [0-2\%]. False negative evaluations revealed $10 \%$ [4-20\%]. Sensitivity and specificity was $37 \%$ [21-57\%] and 99\% [97-100\%] respectively. Median PPV and NPV was both $90 \%$ [60-100\%] and $90 \%$ [78-95\%]. According to percental variations of reference judges' consent, fp-rate, specificity and NPV seems to be more robust than fn-rate, sensitivity and PPV.

Conclusions: Although comparable data of similar documentation systems are missing DokuPIK ${ }^{\circledR}$ seems to have a favorable PPV and NPV to agree with a senior clinical pharmacists ${ }^{\prime}$ majority vote. Despite the allowance of multiple choices, predictive values were good and indicate a well-considered decision making by the pharmacist based on the DokuPIK $^{\circledR}$. The low, but for an exclusive informative tool acceptable sensitivity is explained by a general conservative attitude to recommend more than one possible intervention option by a single pharmacist whereas the reference definition relied on a majority decision. The variability among case reports should be further explored by subgroup analyses.

[1] Ganso 2007 KHP.28:279

Disclosure of Interest: None Declared.

\section{HP-PC108}

Drug information and patients satisfaction in triple therapy for chronic hepatitis $\mathrm{C}$

I. Cañamares-Orbis ${ }^{1, *}$, C. García-Muñoz ${ }^{1}$, J. M. Caro-Teller ${ }^{1}$, I. Escribano-Valenciano ${ }^{1}$, I. Gomez-Valbuena ${ }^{1}$, M. Campo-Angora ${ }^{1}$, J. M. Ferrari-Piquero ${ }^{1}$

${ }^{1}$ University Hospital 12 Octubre, MADRID, Spain

Background and Objective: Recently, two direct antiviral that inhibits replication of hepatitis $\mathrm{C}$ virus (HCV) have been introduced in our hospital. We aim to know the satisfaction of chronic hepatitis $\mathrm{C}$ patients treated with triple therapy (TT), boceprevir or telaprevir, peginterferon and ribavirine, and to determine if exits differences between patients treated with boceprevir and telaprevir, both approved for the same indication.

Setting and Method: In the first visit, the pharmacist provided verbal, written information (individualized leaflet) and practice (injectors demonstration) to patients who had initiated TT in 2012. After they have been completed two months of treatment, they realized a survey (according to HARPS study), in which they punctuated from 1 to 5 his satisfaction with: the facility of administration, numbers of pills, adverse effects (AE), information received, reasons for give-up the TT, etc. The comparison between groups was realized by the non-parametric test of MannWhitney and for the statistical analysis we use SPSS 20.0.

Results: 43 patients that started TT were interviewed (mean age $54.5 \pm 7.7$ years), 24 treated with telaprevir and 19 with boceprevir. We found significant differences in the $\mathrm{AE}$ in alterations of the skin and pruritus, worse tolerated in telaprevir group $(p=0.034) .58 .1 \%$ patients were very satisfied with pharmacist information. $53.5 \%$ patients refer that $\mathrm{AE}$ were well tolerated and a $100 \%$ patients thought that if TT is effective, be worth the effort of toxicity.

Conclusions: Mostly of patients showed to be very satisfied with the information received by the pharmacist. No differences on satisfaction between patients treated with boceprevir and the telaprevir were found. Regarding AE, telaprevir group presented worse dermatological tolerance and irritations coinciding with described with his technical report. Disclosure of Interest: None Declared. 


\section{HP-PC109}

Audit of carbapenems prescription in a health facility, tools to restrict their use

\author{
M. Callanquin ${ }^{1, *}$, M. Callanquin ${ }^{2}$, D. Pozzi ${ }^{1}$ \\ ${ }^{1}$ Pharmacy, ${ }^{2}$ Microbiology, Louis Mourier hospital, Colombes, \\ France
}

Background and Objective: Bacteria can develop more resistant mechanisms because of the abuse of antibiotics prescription. The emergence of carbapenemases and global dissemination represent a significant public health threat. Carbapenem are broad-sprectrum antibiotics that need to be used only for bacteria resistant to other treatments. The main risk is to find a bacterium resistant to all available molecules. The challenge is to succeed in a reasoned and justified use of antibiotics in order to preserve their effectiveness. This is why we have conducted an audit of the carbapenems use in our institution.

Setting and Method: The audit has been conducted over four months, from January to April 2013. Once a week, the investigator (pharmacy resident) was informed of carbapenems deliveries, via the dispensation software and went into the clinical department concerned to identify the patient and complete the information sheet. Then the investigator consulted the patient's record to seek treatment without asking to recover the prescription, avoiding inducing bias. Nominative controlled prescriptions were recovered when they were sent to the pharmacy by services.

Results: 30 imipenem/cilastatin treatments, 2 meropenem and 1 by ertapenem have been identified during the study period. For 17 $(51.5 \%)$ patients, a controlled nominative prescription has been forwarded to the pharmacy. Only for $12(36.4 \%)$ patients that have been treated with carbapenems, ESBL (Extended Spectrum BetaLactamase) has been isolated. Among the 21 carbapenem treatments without ESBL isolation, $12(57 \%)$ have been probabilistic treatments which were stopped after obtaining the antibiogram. 6 (29\%) have been prescribed for undocumented infections: 3 during surgery complications, 2 for nosocomial infections in febrile aplasia contexts, and 1 for community-acquired infections. 3 (14\%) carbapenem treatments without isolation of ESBL instaurations have been motivated by a history of crossing resuscitation or ESBL.

Conclusions: This work has allowed doctors to be aware of the irrational use of carbapenems that can happen. Carbapenems are an effective response against multiresistant bacteria and have to be used only to treat these bacteria. In fact, in $63.6 \%$ of cases the patient has not been infected with ESBL (21 treatments on 33). The carbapenem prescription was unjustified then. A second audit will be conducted in the future to assess the impact of these results on antibiotic prescriptions.

Disclosure of Interest: None Declared.

\section{HP-PC110}

Prescribing psychotropic drugs for the elderly in long-term: study on a given day

V. Falabregues ${ }^{1, *}$, F. Allache ${ }^{2}$, E. Schocher ${ }^{1}$, V. Ducasse ${ }^{2}$, W. Mehtelli ${ }^{3}$, D. Michel ${ }^{1}$, V. Bloch ${ }^{1}$, A. Teixeira ${ }^{2}$, H. Barreteau ${ }^{1}$

${ }^{1}$ Pharmacy, ${ }^{2}$ Gerontology, ${ }^{3}$ Psychiatry, Hospital Fernand Widal, Paris, France

Background and Objective: The prescription of psychotropic drugs is a major problem in geriatrics, especially in long-term care units, which includes a particularly vulnerable elderly population. These treatments were causing an important iatrogenic effects and their indications were not always justified. First of all, we did a psychotropic prescriptions inventory and then, we suppressed inappropriate prescriptions.

Setting and Method: This is a study on a given day. From our software "PHEDRA", we extracted the prescription of each patient: the selection criterion was the presence of at least one psychotropic medication (Benzodiazepine, Neuroleptic, Antidepressant, Anxiolytic or Hypnotic). We analyzed (in pairs: pharmacist-physician) these prescriptions according to tracking sheet treatment of Medical Prescriptions in the elderly of HAS. Medical files and multidisciplinary care records were analyzed.

Main outcome measures: After analyzing prescriptions, therapeutic changes have been made: among 13 patients, the complete cessation of psychotropic prescription could be considered, a dose reduction was done for 4 patients, a molecule changing for 3 patients, a dose adjustment for one patient, an increase in the dose for patients. For one patient, non-drug alternative (psychomotor) has been proposed. In the case of 4 patients with a heavy psychiatric history requiring complex treatment, a specialist opinion was requested to retreat those patients.

Results: On the day of the survey, 77 patients in long-term care units were hospitalised, $50(65 \%)$ of these patients had at least one psychotropic drug prescribed: corresponding to Antidepressants in $60 \%$ of cases, Neuroleptics (36\%), Benzodiazepines (26\%) and Hypnotics $(16 \%): 4$ patients had at least 2 psychotropic drugs. Only one patient had more than 2 associated Benzodiazepines. $56 \%$ of patients had a psychotropic treatment before admission. The prescription's average time was 1,083 days (the average length of stay is 1,335 days). Traceability of the treatment revaluation was present in $52 \%$ of patients.

Conclusions: Our study has highlighted that half of the patients already had a psychotropic medication on the admission's day. As initial indication is often overlooked, adapting these treatments is difficult. These therapeutic changes encourage doctors and pharmacists to collaborate regularly about therapeutic monitoring.

Disclosure of Interest: None Declared.

\section{HP-PC111}

Traceability survey of re-evaluation of antibiotic therapy following the alert introduction by the pharmacist

J. Racaud ${ }^{1, *}$, B. Politis ${ }^{1}$, E. Rouveix ${ }^{2}$, N. Soismier ${ }^{3}$, B. Heym ${ }^{3}$, C. Dupont ${ }^{2}$, A. Dinh ${ }^{2}$, F. Le Mercier ${ }^{1}$

${ }^{1}$ Pharmacy, ${ }^{2}$ Internal medicine, ${ }^{3}$ Microbiology, Ambroise Paré Hospital, Boulogne-Billancourt, France

Background and Objective: The proper use of antibiotics recommends a reassessment of the initial antibiotic therapy within 48-72 h, which should be entered into the patient's medical record. We conducted an evaluation of the impact of the warnings by the pharmacist on the traceability of the reassessment of antibiotic therapy in the medical records by conducting a "before and after" survey.

Setting and Method: The internal medicine department (60 beds) is computerised for one-third of prescriptions (Genois/Phedra ${ }^{\circledR}$ software). Traceability of the reassessment, which can occur between less than $48 \mathrm{~h}$ and $96 \mathrm{~h}$, was sought in patients' medical charts (ORBIS ${ }^{\circledR}$ software)

Main outcome measures: The "before" survey, conducted in June 2012, was retrospective. The "after" survey was prospectively conducted from July to August 2012. The pharmacist's intervention was $48 \mathrm{~h}$ after initiation of antibiotic therapy, and involved sending a message on handwritten prescriptions or establishing a list of patients to reassess for computerised prescriptions. 
Results: The "before" and "after" surveys concerned 23 and 64 patients, respectively. The re-evaluation was recorded in the medical records in $52 \%$ and $67 \%$ of cases before and after the intervention. For the handwritten prescriptions, the re-evaluation was entered in the patient files in $16 \%$ of cases before $48 \mathrm{~h}, 31 \%$ between $48-72 \mathrm{~h}$ and $24 \%$, after this time. It was not entered in $29 \%$ of cases. Reassessment of computerised-prescription antibiotics was found in $19 \%$ of cases before $48 \mathrm{~h}, 27 \%$ between $48-72 \mathrm{~h}$ and $15 \%$ after this time. It was not entered in $39 \%$ of cases.

Conclusions: Traceability of the re-evaluation of antibiotics in the medical record improved significantly $(p=0.0008)$ following the pharmacist intervention, but neither method showed its superiority $(p=0.7606)$. The next priority will be to evaluate the impact of implementation of an automatic message on the antibiotic's information in the prescription software (ORBIS $\left.{ }^{\circledR}\right)$.

Disclosure of Interest: None Declared.

\section{HP-PC112}

Indications of oral Clonazepam prescriptions in a pediatric hospital

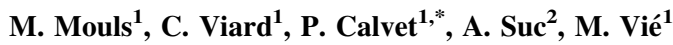 \\ ${ }^{1}$ Service of pharmacy, ${ }^{2}$ Service of pediatric pain, CHU Toulouse, \\ Toulouse, France
}

Background and Objective: Clonazepam is a benzodiazepine indicated for the treatment of epilepsy. Despite information by ANSM on the proper use of Clonazepam distributed to prescribers in 2008, this drug is widely prescribed off-label. News conditions for prescribing and dispensing were issued in 2011 and in 2012 including the restriction of the initial prescription and annual renewal for neurologists and pediatricians. The objective of this study was to evaluate the indications of oral Clonazepam prescriptions in a pediatric hospital.

Setting and Method: We analyzed the prescriptions of Clonazepam between February 11 and April 8, 2013 in 9 hospitalization units (neurology, hematology-oncology, surgery, intensive care...) of a pediatric hospital. We collected data from patients' records and interviews with prescribers. For the analysis, our reference information folders were drug "RCP" and new recommendations for the proper use released in December 2012 by ANSM.

Results: We identified 30 prescriptions ( 28 children) during the audit period. All prescriptions have been initiated in thé hospital and $57 \%$ of them are primary prescriptions. Clonazepam was prescribed offlabel in $73 \%$ of cases including neuropathic pain $(53 \%)$ and iatrogenic pain post chemotherapy ( $32 \%)$. The average dosages used vary from 0.05 to $0.1 \mathrm{mg} / \mathrm{kg} / \mathrm{day}$ depending on the indication. All of Clonazepam prescriptions for pain treatment are associated with painkillers. In the context of neuropathic pain was also found an association with antiepileptic drugs such as gabapentin or pregabalin. Conclusions: This work shows that Clonazepam is still widely prescribed off-label in children because of its possible initiation by all pediatricians. The main indication is the treatment of neuropathic pain in children. Besides the fact that all treatment use in adults have no authorization to be marketed in children for this indication (amitriptyllin, gabapentin, pregabalin) and therefore not in a suitable dosage form. The time of action of antiepileptic and antidepressants used in this indication allow an average of 7 to 15 days, which does not handle the pain quickly. In most cases Clonazepam is prescribed occasionally and, pending the effectiveness of recommended molecules, chemotherapy-induced pain. Treatments with Clonazepam for neuropathic pain are systematically initiated after consultation with the nurses/doctors specializing in pain in children.

Disclosure of Interest: None Declared.

\section{HP-PC113}

Interaction program implementation for pulmonary hypertension patients taking oral treatment

M. Vázquez-Real ${ }^{1, *}$, S. Sandoval Fernández del Castillo ${ }^{1}$, M. D. Alvarado-Fernández ${ }^{1}$, S. Santana Martínez ${ }^{1}$, E. Romero Carreño ${ }^{1}$, J. A. Marcos Rodríguez ${ }^{1}$

${ }^{1}$ UCG Farmacia, Hospital Universitario Virgen Macarena, Sevilla, Spain

Background and Objective: Endothelin receptor antagonists (ERA) and phosphodiesterase type 5 inhibitors (PDE5I) are common drugs to treat pulmonary hypertension $(\mathrm{PH})$. Avoiding interactions with home medications improve the results and life quality of patients. The aim is to implement an interaction program for patients with oral treatment for PH in collaboration with cardiologist and primary doctors in order to improve the effectiveness of treatment and reduce adverse drug reactions (ADR).

Setting and Method: Patients getting sildenafil and tadalafil (PDE5I) or bosentan and ambrisentan (ERA) from the hospital's pharmacy during the last year were selected. Main interactions were categorised into high, medium and low risk according to recommendations recorded at Lexicomp ${ }^{\circledR}$, Medscape ${ }^{\circledR}$, Micromede $^{\circledR}$ and Drugs.com. Home medication data was obtained by Diraya ${ }^{\circledR}$ application. An Excel ${ }^{\circledR}$ table was elaborated to cross the information.

Results: There were 42 patients in treatment. 16 (39\%) were males and $25(61 \%)$ were females. 25 patients $(59 \%)$ were taking sildenafil, $1(2 \%)$ taladafil, 5 (12\%) ambrisentan and $11(27 \%)$ bosentan. 67 drug interactions were identified for sildenafil, 43 for tadalafil, 10 for ambrisentan and 55 for bosentan. 7 patients presented a relevant interaction. The next step was to contact to the primary doctor and discuss an alternative to the related drug. Last, for all patients there was made an informative diptych addressed to the cardiologist and primary doctor to inform about the most important interactions and treatment alternatives.

Conclusions: Drug interactions may provoque treatment failure or ADR. The elaboration of a plan in order to identify potencial interactions makes hospital pharmacist to participate improving patient's treatment. This initiative has been widely accepted by clinicians and patients.

Disclosure of Interest: None Declared.

\section{HP-PC114}

Retrospective analysis of antifungal agents use in pediatric patients with cancer

P. Gantner ${ }^{1, *}$, A. Spiegel ${ }^{2}$, M.-O. Duzanski ${ }^{1}$, A. El Aatmani ${ }^{1}$, D. Levêque ${ }^{1}$, N. Entz-Werlé2 ${ }^{2}$ P. Lutz ${ }^{2}$, B. Gourieux ${ }^{1}$

${ }^{1}$ Pharmacy/Sterilization department, ${ }^{2}$ Pediatric Hematology/ Oncology department, Teaching Hospitals of Strasbourg, STRASBOURG, France

Background and Objective: This study aimed to analyze the use of antifungal agents in pediatric patients with cancer, based on labelling and clinical guidelines.

Setting and Method: Retrospective study of antifungal agents prescription in patients hospitalized in the pediatric hematology/oncology department of Strasbourg's teaching hospital, France. A physician and a pharmacist examined antifungal prescription according to the French labelling and the European guidelines (ECIL-4).

Main outcome measures: Pattern of use of antifungal therapy; analysis of indication and dosage in relation with the French labelling and the European (ECIL-4) guidelines. 
Results: Between January and December 2011, 82 antifungal prescriptions of 32 children (median age: 12; range 1-21 years) were analyzed. Children had haematological malignancies (n:23), solid tumours (n:8) and drug-induced agranulocytosis (n:1). Half of the children had undergone hematopoietic stem cell transplantation (70\% allogeneic stem cell transplantation). Most (96\%) of the prescriptions concerned antifungal given as single agent. They were administrated as prophylaxis $(n=43)$, empirical therapy $(n=27)$ and pre-emptive treatment for possible invasive aspergillosis $(\mathrm{n}=12)$. Caspofungin accounted for $43 \%$ of prescriptions, followed by posaconazole $(30 \%)$, liposomal amphotericin B (16\%), fluconazole $(7 \%)$ and association of liposomal amphotericin B with caspofungin $(4 \%)$. Regarding indication, the adherence to labelling and the ECIL-4 guidelines were $77 \%$ and $82 \%$, respectively. The most off-label prescribed drug was caspofungin $(11 \%$ of the prescriptions) used as prophylactic therapy. The adherence to dosing recommendations was $74 \%$. Inappropriate dosing was primarily due to the lack of loading dose in 13 caspofungin prescriptions. ECIL-4 recommendations regarding optimization of posaconazole therapy with therapeutic drug monitoring were not applied due to the lack of routine analytical support.

Conclusions: The adherence to labelling and European guidelines in what regards indication and dosing was similar to a previous french study (80\%; Berthe A. and al. Med Mal Infect, 2011). Most of deviations concerned caspofungin given off label in prophylaxis and without loading dose at day 1 .

Disclosure of Interest: None Declared.

\section{HP-PC115}

Antibiotherapy reassessment after 48-72 $\mathrm{h}$ in hospitalized geriatric patients and impact of pharmaceutical interventions

\section{Peloso $^{1,{ }^{*},}$ C. Ourghanlian ${ }^{1}$, C. Michel $^{1}$, R. Ratiney ${ }^{1}$ \\ ${ }^{1}$ Pharmacy, René Muret Hospital AP-HP, Sevran, France}

Background and Objective: Infectious diseases are the third cause of elderly mortality. Antibiotic treatment must be effective, warranted, well-dosed and systematic reassessment after 48-72 h ensures efficacy. The aim of this study is to evaluate antibiotherapy reassessment in hospitalized geriatric patients and impact of pharmacist involvement.

Setting and Method: The study is prospective with 2 inclusion periods. Period 1: All patients hospitalized in geriatric units (400 beds) with antibiotic treatment were included in an observational study. Period 2: Patients hospitalized in a geriatric "test unit" (35 beds) with specific pharmaceutical interventions (PIs) (electronic message, phone call or face-to-face) were included in an interventional study. Patients were identified by computerized prescription and data were collected in patient medical records. Type of infection, treatment choices, respect of local protocols, bacteriological results, renal function and reassessment criteria and consequences were analysed.

Main outcome measures: Effectiveness of antibiotherapy reassessment (period 1) and impact of PIs (period 2).

Results: Period 1: 79 patients were included (mean age 84 years-old) during the obervational period (01.02.2013 to 01.15.2013). Infections were mainly located in pulmonary $(37 \%)$ and urinary $(30 \%)$ tract. Other infections are septicemias (6\%), cutaneous (6\%), ENT (5\%) and digestive $(3 \%) .72 \%$ of antibiotherapy followed local protocols. Antibiotherapy was initially provided by injectable $(43 \%)$ or oral route $(57 \%)$. Recent creatinine value was available for $84 \%$ of patients. When required, a dosage adjustment for renal impairment followed the recommendations $(87 \%)$. Bacteriological samples were achieved for $62 \%$ of patients. Reassessment was based on bacteriological results $(40 \%)$, biological and clinical criteria. Outcomes were: continuing $(73 \%)$, stopping $(8 \%)$ or changing $(19 \%)$. Period 2: During the period with PIs (06.10.2013 to 06.21.2013), 18 patients (mean age 84 years-old) were included with mainly pulmonary $(50 \%)$ or urinary $(30 \%)$ infections. Antibiotic treatments (initially injectable $(56 \%)$ or oral $(44 \%)$ ) followed local protocols $(77 \%)$. Recent creatinine value was available for $94 \%$ of patients, leading to 4 dosage adjustements. Bacteriological samples were performed in $50 \%$ of cases. Reassessment was mainly based on clinical and biological criteria. Reassessment outcomes, including 2 based on PIs, were: continuing $(72 \%)$, stopping $(17 \%)$ or changing $(1 \%$, switch from injectable to oral route) treatment.

Conclusions: Reassessment of antibiotherapy has to be performed 48-72 $\mathrm{h}$ after initiation, but bacteriological data are often missing and treatments are mainly empirical. This study shows that PIs are effective to stop unjustified treatments. To confirm these results, inclusion of more patients is needed. PIs should be extended to other geriatric units but this first stage already shows pharmacists's place near physicians and biologists in antibiotic treatment reassessment. Disclosure of Interest: None Declared.

\section{HP-PC117}

\section{Impact and role of pharmacists in surgery}

A. Guérin ${ }^{1, *}$, D. Lebel ${ }^{1}$, J.-F. Bussières ${ }^{1}$

${ }^{1}$ Pharmacy, CHU Sainte Justine, Montréal, Canada

Background and Objective: A high level of care is required in surgery and many pharmacotherapy adjustments are necessary, such as the start of anticoagulotherapy, anaesthesia, pain medication and antibiotic prophylaxis. Decentralized pharmacists in surgery programs are essential. The aim of this study was to review the literature on the impact and the role of pharmacists in surgery.

Results: A Web portal about the evidences of the impact and the role of pharmacists in specific diseases, programs of care or pharmaceutical activities was developed. A structured approach to select, review, summarize and eventually diffuse the information to the healthcare community was developed. A literature search on Google and Pubmed $^{\circledR}$ was conducted: pharmacist Mesh AND surgery All fields; pharmacist Mesh OR clinical pharmacist Mesh OR pharmaceutical care Mesh AND hospital surgery department Mesh. French and English articles about the role and the impact of pharmacists in surgery from 1990 to 2013 were included. Graft-related articles were excluded. A total of 179 articles were initially identified. Fifteen relevant articles were included in our analysis. For each include article, key indicators that document the impact of pharmacist with statistical analysis and the role of pharmacists with only quantitative or qualitative metrics were identified. All relevant pharmaceutical activities in that context were also identified.

From the 15 included studies about the role and the impact of pharmacist in surgery, 25 key indicators of the impact were identified. There was a positive impact for 18 indicators, such as pain, International Normalized Ratio, glycemic control, number of missed doses per patient, medication discrepancy, medication charted at an incorrect dose or frequency, length of antimicrobial use and intraveinous use and time of switch from intraveinous to per os. Fifty-eight key indicators about the role of pharmacists were identified. Pharmacists were providing drug therapy evaluation, patient counseling, medication reconciliation, education, drug information, pharmacokinetics consultation and participation to medical rounds.

Conclusions: There are limited data published about the role of pharmacists in surgery. While it seems relevant to support such clinical implication, pharmacists involved in that program of care should better document and evaluate their impact.

Disclosure of Interest: None Declared. 


\section{HP-PC118}

Impact and role of pharmacists in parenteral nutrition

\author{
A. Guérin ${ }^{1, *}$, D. Lebel ${ }^{1}$, J.-F. Bussières ${ }^{1}$ \\ ${ }^{1}$ Pharmacy, CHU Sainte Justine, Montréal, Canada
}

Background and Objective: The American Society for Parenteral and Enteral Nutrition defined that pharmacists have a role in the management of specialized nutrition support programs and in the improvement of quality. The aim of this study was to review the literature on the impact and the role of pharmacists in parenteral nutrition.

Results: A Web portal about the evidences of the impact and the role of pharmacists in specific diseases, programs of care or pharmaceutical activities was developed. A structured approach to select, review, summarize and eventually diffuse the information to the healthcare community was developed. A literature search on Pubmed ${ }^{\circledR}$ was conducted: pharmacist Mesh OR clinical pharmacy service Mesh OR pharmaceutical care Mesh AND parenteral nutrition Mesh. French and English articles about the role and the impact of pharmacists in parenteral nutrition from 1991 to 2013 were included. A total of 223 articles were initially identified. Nine relevant articles were included in our analysis. For each article included, all key indicators that document the impact of pharmacist with statistical analysis and the role of pharmacists with only quantitative or qualitative metrics were included. All relevant pharmaceutical activities in that context were identified.

From the nine studies included about the role and the impact of pharmacist in parenteral nutrition, 13 key indicators of the impact were identified. Key indicators included the calories and proteins intake, the number of laboratory tests per patient per day and the proportion of laboratory values in the appropriate range for chloride, bilirubin, blood urea nitrogen, creatinine, glucose, phosphorus, magnesium, bicarbonates, sodium and potassium. Four of the 13 indicators showed a positive impact of the pharmacist in cystic fibrosis. Forty-one key indicators about the role of pharmacists were also identified. Amongst the pharmaceutical activities, pharmacists were providing evaluation of the drug use, drug therapy evaluation, drug therapy monitoring, patient counseling, education outside and within the pharmacy department, risk prevention and participation in medical rounds.

Conclusions: There are limited data published about the role of pharmacists in parenteral nutrition. While it seems relevant to support such implication, pharmacists involved in that program of care should better document and evaluate their impact.

Disclosure of Interest: None Declared.

\section{HP-PC119}

Potentially inappropriate medications for elderly: study of $\mathbf{2 0 0}$ prescriptions of a geriatric's pole

\section{A. Schwarzenbart ${ }^{1, *}$, B. Michel ${ }^{1}$, G. Kaltenbach ${ }^{2}$, B. Gourieux ${ }^{1}$ \\ ${ }^{1}$ Pharmacy department, ${ }^{2}$ Geriatry department, Hôpitaux Universitaires de Strasbourg, STRASBOURG CEDEX, France}

Background and Objective: The use of inappropriate medication in older patients at the University Hospital of Strasbourg was unclear. This study was therefore set out to analyse the frequency and patterns of potentially inappropriate drug prescriptions in elderly.

Setting and Method: This prospective study was conducted in August 2012 in four different medical units of the geriatrics' department (466 beds). The first fifty patients of each unit, aged 75 years and older, were included.
Main outcome measures: To describe the trends of potentially inappropriate medication (PIM) use, the 200 prescriptions collected were analyzed according to Beers, Laroche, Priscus criteria and the US update. Results: Among the 200 elderly patients, a majority of the sample was female: sex ratio was 0.28 , average age was 87 years old, and the average number of drugs per prescription was 8.3 (extreme: 1-18). In total, 1,657 drugs were prescribed for these 200 patients among them 252 were PIMs (15\%). Overall, $74 \%$ of the patients had at least one PIM and the average number of PIMs per patient was 1.7 (extreme: $0-7)$. The most commonly used PIMs were psychotropic (54\% [137/ 252]), antithrombotic (23\% [59/252]) and laxative medications (6\% [14/252]). These findings show that the use of PIMs is common among the elderly in our hospital, particularly one class: psychotropic. In order to reduce the proportion of PIMs given to the elderly, multidisciplinary and quality circles groups are currently working on specifics guidelines.

Conclusions: This evaluation in geriatrics' department allowed to initiate a collective reflection on prescription in hospitalized older patients. The use of PIMs can also be debated taking into consideration the frailty of the patients.

Disclosure of Interest: None Declared.

\section{HP-PC120}

\section{Use of benzodiazepines in elderly patients}

V. Saavedra Quirós ${ }^{1, *}$, A. Díez Alcántara ${ }^{1}$, F. Á. Ortega Montalbán $^{1}$, E. Montero Hernández ${ }^{2}$, A. Torralba Arranz ${ }^{1}$

${ }^{1}$ Pharmacy, ${ }^{2}$ Internal Medicine, Hospital Universitario Puerta de Hierro, Majadahonda, Spain

Background and Objective: Community level studies have shown that, up to a $25 \%$ of people older than 75 years take benzodiazepines (BDZs) at some point in their lives. The BZDs use may cause excessive sedation, cognitive impairment, psychomotor and coordination disorders, slowing-down, falls (with the risk of fractures)..., elderly being more sensitive to these adverse effects.

The goal of our study was to evaluate the use of BDZs in elderly patients included in a medication reconciliation program and to analyze the correlation between the use of BDZs and other drugs which are known to favor the falls (other sedative drugs, antipsychotics and opioids), and bone fractures.

Setting and Method: Retrospective observational study of 565 patients reconciled at admission in 2012. The profile of the patients was: elderly over 75 years-old, polymedicated (6 or more different drugs), living in nursing homes.

Main outcome measures: The parameters analyzed were: sex, age, number of different drugs prescribed, number of patients taking BZDs or any of the other drugs previously mentioned, and number of patients admitted due to bone fracture.

Results: Of 565 patients reconciled (70.7 \% women), 240 (42.5\%) were receiving treatment with at least one benzodiazepine, and if we considered other drugs related with falls, the number of patients increases up to the $67 \%$. The average age was 86.9 (75-101 years) and the average number of drugs as usual treatment was 10 (6-21 drugs). In $48(8.5 \%)$ out of the 565 patients, the diagnosis at admission was bone fracture ( $83.3 \%$ women). Although the $50 \%$ of the patients admitted due to bone fracture were taking at least one BDZ ( $71 \%$ short half-life BDZs), and up to the $77 \%$ if we consider one of the other drugs too, the statistical analysis did not revealed significant difference in the incidence of bone fractures related with the intake of this type of drugs.

Conclusions: Our study reveals a consumption of benzodiazepines in elderly patients higher than other studies. 
The results are not statistically significant, perhaps due to the small size of sample, so further studies could evaluate the falls or fractures suffered in the 3 months previous to the admission date.

Due to the fact that this study is performed in a medication reconciliation program, we consider essential to analyze the risk/benefit balance of continuing treatment with this type of drugs at the time of medication reconciliation at hospital discharge.

Disclosure of Interest: None Declared.

\section{HP-PC121}

Impact of clinical pharmacist intervention with infectious disease team

\section{E. T. Almadhoun ${ }^{1, *}$}

${ }^{1}$ Clinical pharmacy, Hamad General Hospital, Doha, Qatar

Background and Objective: To determine the impact and cost saving of clinical pharmacist's intervention with infectious disease team, regarding treatment selection, dosing, renal and hepatic adjustments, Therapeutic drug monitoring and patient follow up.

Setting and Method: 1. This is a prospective observational study from February 2010 to June 2012.In this study the clinical pharmacist daily rounded with infectious disease team, make and documents interventions for patients in medical, surgical and intensive care units.

2. the Clinical pharmacist interventions includes treatment selection, appropriate dose, renal and hepatic dose adjustments, TDM and patient education and follow up for any side effects.

3. All intervention data were entered and analyzed using SPSS program version 20 .

Main outcome measures: To determine the impact and cost saving of clinical pharmacist's intervention with infectious disease team.

Results: Over more than 700 rounding days the clinical pharmacist made over than 950 interventions. $49 \%$ of interventions related to drug dosing, $16.2 \%$ of interventions dealing with determining the duration of therapy, $12.7 \%$ related to writing prescriptions and $7.5 \%$ for therapeutic drug monitoring. Some of interventions data were lost because the unavailability of SPSS program during months June, July and August 2011.

Conclusions: The clinical pharmacist has a huge impact and great role with infectious disease team leading to better patient care and optimal outcomes.total number of intervention were more than 950 clinical interventions over 700 rounding days.this study empathizes on the great role of clinical pharmacist with recommendation to hire more clinical pharmacists to cover other specialty teams .

Disclosure of Interest: None Declared.

\section{HP-PC122}

High-alert medications in pediatrics hospital

J. Bataille ${ }^{1,2, *}$, P. Joret ${ }^{1,2}$, S. Prot-Labarthe ${ }^{1}$, O. Bourdon ${ }^{1,2,3}$, F. Brion ${ }^{1,2,3}$, J.-F. Hartmann ${ }^{4}$

${ }^{1}$ APHP, Pharmacie, Hôpital Robert-Debré, ${ }^{2}$ Pharmacie Clinique, Université Paris Descartes, Paris, ${ }^{3}$ Laboratoire Education et Pratiques de Santé, Université Paris XIII, Bobigny, ${ }^{4}$ Coordonnateur de la gestion des risques associés aux soins, CLIN/CVRiS, Responsable du management de la qualité de la prise en charge médicamenteuse, Hôpital Robert-Debré, Paris, France

Background and Objective: High-alert medications have the highest risk of causing injury when misused. Errors on high-alert medications occur at the same rate compared to usual medicine. Pediatric population itself is at high risk of drug misuse. The aim of this study is to identify clinicians, nurses and pharmacists perceptions of high-alert medications and to develop a pediatrics specific high-alert medications list. Setting and Method: A three step process was set: (1) review of literature, (2) electronic survey was sent to clinicians (seniors and residents), registered nurses, young nurses and pharmacists working in a pediatric university French hospital. They suggested their top ten highalert medications and the medication which was the most frequently involved. They were asked the type of errors they have already encountered and to describe the consequences of the misuse. Results were compared to the Institute for Safe Medications Practices list and Franke \& al PICU-specific, high-alert medications list. (3) consensus of experts who approved a list of high-alert medications for the hospital. Main outcome measures: Obtain a pediatrics specific high-alert medications list.

Results: The review of literature pinpoints four documentary sources, 27 medication classes and 63 specific medications. Only one study has identified a pediatric intensive care unit, specific high-alert medications in the United States (US). The response survey rate was $20 \%$ and nurses were the most represented category. The number of medicines listed fluctuate form 3-10. The consensus list was composed of 17 medication classes and specific medications such as adrenaline aerosol, amiodarone, anticoagulants and antimalarial drugs. The complete table will be presented in the final poster.

Conclusions: Four medications weren't identified on the Institute for Safe Medications Practices list and the PICU-specific, high-alert medications list: injectable acetaminophen, quinine and derivates, magnesium sulfate and adrenaline aerosol. This difference could be due to diversity of practice between France and US (incidence of pathology, habit of care and specific commercialized medicine). Currently, no pediatrics' specific high-alert medications list was described in France and only one in the US. The diffusion of the list can sensibilise the nursing staff to the drugs they have to be aware of. A national study has to be conducted in other pediatrics' hospital to confirm these results.

Disclosure of Interest: None Declared.

\section{HP-PC123}

Qualitative evaluations of antibiotics use with Gyssens method in department of child health, Dr. Cipto Mangunkusumo hospital

\section{R. Andrajati ${ }^{1, *}$, D. S. Pamela ${ }^{1}$, R. Mutiara ${ }^{2}$}

${ }^{1}$ Pharmacology, Pharmacy Faculty, Universitas Indonesia, Depok, ${ }^{2}$ Pediatric, Cipto Mangunkusumo Hospital, Jakarta, Indonesia

Background and Objective: To evaluate the intervention of pharmacists on quality of antibiotic use in Class 3 Infection Ward, Department of Pediatrics, Cipto Mangunkusumo Hospital.

Setting and Method: An intervention prospective study using descriptive-correlative approach using Gyssens method in Class 3 Infection Ward, Department of Pediatrics, Cipto Mangunkusumo Hospital, Indonesia.

Main outcome measures: The quality of antibiotic use and intervention of pharmacist.

Results: A high proportion (78.82 \%) of 170 patients received antibiotics from January to April 2011. Qualitative evaluation using Gyssens method had result that about $60.4 \%$ antibiotic prescriptions were appropriate; and $39.6 \%$ were inappropriate. Intervention of pharmacist improved appropriateness of antibiotics (from 0 to $67.1 \%$ ), decreased timing problems (from 32.9 to $0 \%$ ), dosage problems (from 27.4 to $19.2 \%$ ), duration problems (from $5 / 5$ to $2.7 \%$ ), drug choice problems (from 32.9 to $11 \%$ ) and indication problems (1.4 to $0 \%$ ).

Conclusions: Antibiotics were prescribed mostly inappropriate. Intervention of pharmacist could improve quality of antibiotic use. Disclosure of Interest: None Declared. 


\section{HP-PC124}

Impact and role of pharmacists in geriatric

\author{
A. Guérin ${ }^{1, *}$, D. Lebel ${ }^{1}$, J.-F. Bussières ${ }^{1}$ \\ ${ }^{1}$ Pharmacy, CHU Sainte Justine, Montréal, Canada
}

Background and Objective: Geriatrics is the study of aging, its physiological phenomena, and diseases common with older people. Trends in population ageing have been noticed in Europe for many years. The aim of this study was to review the literature on the impact and the role of pharmacists in geriatric.

Results: A Web portal about the evidences of the impact and the role of pharmacists in specific diseases, programs of care or pharmaceutical activities was developed. A structured approach was developed to select, review, summarize and eventually diffuse the information to the healthcare community. A literature search on Pubmed ${ }^{\circledR}$ was conducted. Articles about the role and the impact of pharmacists in geriatric in French and English from 1990 to 2013 were included. Ninety seven relevant articles were included in our analysis. For each article included in the analysis, key indicators that document the impact of pharmacist with statistical analysis and the role of pharmacists with only quantitative or qualitative metrics were identified. All relevant pharmaceutical activities in that context were also identified.

From the 97 included studies about the role and the impact of pharmacist in geriatric, 228 key indicators of the impact were identified: drug costs saving from recommendations, adverse effects, quality of life, falls, mobility, confusion, hospital readmission, number of deaths, adherence, satisfaction, mean number of medications, knowledge of medicines, etc. Sixty-nine of the 228 indicators showed a positive impact of the pharmacist in geriatric. One-hundred key indicators about the role of pharmacists were identified. Amongst the pharmaceutical activities, pharmacists were providing medication reconciliation, patient counseling, teaching, drug therapy evaluation, drug information, multidisciplinary meeting, drug prescription conformity follow-up, and medical rounds.

Conclusions: Geriatrics is a healthcare program where the role and impact of pharmacists are well documented. Pharmacists had a positive impact with activities such as reducing the number of medication taken and improving drug dosage. Pharmacists involved in geriatrics should better document and evaluate their impact on activities such as medication errors. The high number and variability in the type of key indicators used limit comparisons between studies. Disclosure of Interest: None Declared.

\section{HP-PC125}

Check list sheet to standardize pharmaceutical care in chronic kidney disease patients during hospital care

L. Castillo Palomares ${ }^{1, *}$, L. Canadell Vilarrasa ${ }^{1}$, J. Torrent Pou ${ }^{1}$, M. Martín Marques ${ }^{1}$, M. Canela Subirada ${ }^{1}$

${ }^{1}$ Farmacia, Hospital Universitario Joan XXIII, Tarragona, Spain

Background and Objective: Rational drug prescription is a difficult task in chronic kidney disease (CKD) patients because they are at higher risk of drug related problems since they need complex therapeutic regimens that require frequent monitoring and dosage adjustments. In addition they usually have other morbidities including diabetes mellitus, hypertension, coronary artery disease and infection. The optimal attention of these patients requires a pharmaceutical care program to assess the therapeutic plan to identify all drug related problems (DRP). In order to simplify this activity, we proceeded to establish a check list sheet to optimize patient management and standardize pharmaceutical care.
Setting and Method: We evaluated all of CKD patients admitted to nephrology unit during six months: medical history, comorbidities, dialysis information and drug therapy. Then, it was reviewed the national guidelines of the problems related to CKD patients and the optimal drug treatment. Finally, we created a management check sheet to be used by pharmacists in order to standardize the daily pharmaceutical care activity.

Main outcome measures: The mean number of comorbid conditions of our DRP patients was $3.2 \pm 1.5$. The mean number of medication used during hospitalization was $10.5 \pm 3.7$ More than $94 \%$ of the patients it was prescribed 5 or more drugs.

The check list sheet contains the major medical problems of these patients, the evolution indicators, the standard drug treatment approved in our hospital and the drug monitoring parameters of security.

Results: The check list sheet includes the management of the following medical problems: electrolyte imbalance, acid-base imbalance, metabolic disorders, hypertension, correction of nutritional status, dosage adjustments in renal impairment and with the presence of dialysis techniques, chronic anemia, uremia, and lipid disorders.

Conclusions: It is necessary to establish protocols for pharmacists to guarantee the quality of the activity, the standardization and the optimization of pharmaceutical resources and the development of a check list is a simple method to obtain it.

Disclosure of Interest: None Declared.

\section{HP-PC126}

The patient's medication experience as the root of drug therapy problems in patients with type 2 diabetes

\section{P. M. Aguiar, ${ }^{1,}$, G. C. Brito ${ }^{2}$, D. P. Lyra Jr. ${ }^{2}$, S. Storpirtis ${ }^{1}$}

${ }^{1}$ Faculty of Pharmaceutical Sciences, University of São Paulo, São Paulo, ${ }^{2}$ College of Pharmacy, Federal University of Sergipe, São Cristóvão, Brazil

Background and Objective: In order to ensure effective, patientcentered medication management, the pharmacists should incorporate the perspectives and needs of patients into their clinical judgment and decision-making processes. The medication experience is different for each individual patient and can shape patients' behaviors regarding their medications; it might lead to the development of drug therapy problems (DTPs). Thus, the purpose of this study was to identify the DTPs rooted in patients' medication experiences.

Setting and Method: A 10-month prospective study of medication management was conducted at Metabolic Diseases Clinic, University Hospital of São Paulo, Brazil. Patients were enrolled if they had been diagnosed with type 2 diabetes, were aged 40 to 79 years and presented glycosylated hemoglobin greater than $7 \%$. The pharmaceutical interviews were conducted face-to-face after the medical appointments, along with remote support by telephone. Pharmacist performed monitoring of DTPs and examined if the patients' medication experiences (e.g., values, desires, and fears associated with care and treatment) could influence their development. The most likely causes of DTPs, as well as possible solutions, were discussed with each patient.

Main outcome measures: The number of DTPs rooted in patients' medication experiences identified/resolved by the clinical pharmacist. Results: Of the 40 patients enrolled, the mean age was 61.5 years ( \pm 7.7 ), and $67.5 \%$ were female. All patients presented at least one DTP. A total of 143 DTPs were documented and 49 (34.3\%) of these were rooted in patients' medication experiences. The adherence was the DTP more frequently $(36 ; 73.5 \%)$ related with subjective experiences reported by patients, such as doubts the need for the 
medications $(21 ; 42.9 \%)$ and does not like to take medications (10; $20.4 \%)$.The main clinical pharmacist's interventions consisted of educate on health condition and/or medication and work to prioritize health goals, sharing how the medication can fit those goals. Of the total number of these DTPs, $39(79.6 \%)$ were resolved through dialogue and agreement with the patient.

Conclusions: In our study, the medication experience influenced patients' attitudes and medication-taking behavior and it may be sometimes considered the root cause of DTPs. The understanding of the medication experience was vital to assess patients' needs and for planning interventions in the care process.

Disclosure of Interest: None Declared.

\section{HP-PC127}

Medication reconciliation: a process for optimizing patient care

D. Provenzano ${ }^{1, *}$, A. Carollo ${ }^{2}$, I. Casucci ${ }^{2}$, P. Polidori ${ }^{2}$

${ }^{1}$ School of Specialization in Hospital Pharmacy, University of Palermo, ${ }^{2}$ Clinical Pharmacy, ISMETT, Palermo, Italy

Background and Objective: Medication Reconciliation (MR) is a multidisciplinary process aimed at improving patient safety through checking of drug prescriptions on admission and at discharge. The purpose of the study was to verify the correctness of drug treatment prescribed at discharge/called first cycle of therapy by means MR performed by clinical pharmacist $(\mathrm{CP})$ to prevent discrepancies and omissions of drugs.

Setting and Method: A 17-months retrospective observational study, from January 2012 to May 2013, was conducted by ISMETT's Clinical Pharmacy Service. We compared patients' FCT prescriptions with orders prescribed in the electronic medical record (EMR) and reported in the discharge letter. Incorrect FCT prescriptions were deleted during the MR and the FCT dispensing process. MR discrepancies and omissions were recorded and discussed with the prescriber for a rapid resolution.

Main outcome measures: Identification of discrepancies, through MR, omissions and errors in drug therapy prescribed at discharge as FCT by comparing the prescriptions in the discharge letter and in the EMR.

Results: We reviewed 6,350 prescriptions at hospital discharge, 257 of which (4\%) were deleted because incorrect, and $393(6.2 \%)$ because discordant with patient's in-hospital drug therapy. In total, we intervened in $650(10.2 \%)$ FCT drug prescriptions.

We deleted 257 prescriptions for the following reasons: 119 (46.3\%) incorrect dispensing regimen; $70(27.2 \%)$ non-conformity with law n.405/2001 and amendments and additions; 50 (19.5\%) incorrect drug therapy; 9 (3.5\%) medication prescribed for wrong patient; 5 (1.9\%) duplicate drug; 4 (1.6\%) off-label drug.

Of the 393 discrepancies, $211(53.7 \%)$ were prescriptions for the different drug, though of the same therapeutic class; 132 (33.6\%) prescriptions found in the FCT, but not in the EMR; 31 (7.9\%) different therapeutic class, most of which were antibiotics and hypoglycemic drugs; 19 (4.8\%) different pharmaceutical form.

Conclusions: The $\mathrm{CP}$, through the MR process, supports the physician by critically reviewing the medications prescribed as FCT, and at hospital discharge, thus making the patient's home drug-therapy safer. The MR at discharge is a necessary tool for preventing medication errors as patients move from care setting to home, and helps ensure continuity of care and efficient coordination among different levels of care.

Disclosure of Interest: None Declared.

\section{HP-PC128}

Relevance of documentation sources used to establish medication reconciliation in surgery departments

V. Hazard ${ }^{1, *}$, C. Serrano ${ }^{1}$, M.-C. Chaumais ${ }^{1}$, S. Foucher ${ }^{1}$, A. Rieutord ${ }^{1}$, S. Roy ${ }^{1}$

${ }^{1}$ Pharmacy, Hospital Antoine Béclère, Clamart, France

Background and Objective: Since 2011, about 150 medications reconciliations (MR) are monthly performed by pharmacy students in surgery departments. MR is usually based on at least 2 sources of information including the patient's interview. Completion rate is expected to be at least of 70 and $90 \%$ at 24 and $72 \mathrm{~h}$ after admission respectively. The aim of this study was to evaluate the completion rate and the relevance of information sources (IS) used to perform MR.

Setting and Method: During a one month prospective study, we recorded IS used to carry out MR. We considered 4 sources relevant (patient's general physician, retirement home, the prescription and the patient interview) and 6 sources insufficiently relevant (hospitalisation report $<3$ months, clinical letter, anaesthesia file, drugs brought by the patient, patient's family and emergency's report). All inpatients of the surgery's wards were included. Results were analysed depending on 3 risk factors (RF): age $\geq 65$ years, at least 2 chronic pathologies or at least 3 long-term drugs.

Main outcome measures: For each patient: RF status and number of relevant and irrelevant sources used to establish the MR. Completion rate of MR.

Results: 129 patients were included with a mean age of $56.6 \pm$ 19.8 years, 1.4 chronic pathologies and 3.4 long-term treatments were reported per patient. 3 patients were excluded due to a lack of source reported. Completion rates at 24 and $72 \mathrm{~h}$ were $49.2 \%$ and $85.7 \%$, respectively. Among the $126 \mathrm{MR}, 100$ (79.4\%) were based on at least 2 sources and $26(20.6 \%)$ upon only 1 source. However, regarding the sources relevance, $8.7 \%$ of MR were done without any source, $70.7 \%$ were based on 1 source and $20.6 \%$ on 2 sources. Furthermore, among the 29 patients with 3 RF: 3 $(10.3 \%), 14(48.3 \%), 12(41.4 \%)$ had MR were done with 0,1 or 2 sources, respectively. For the 41 patients with 1 or 2 RF: 2 (4.9\%), $28(68.3 \%)$, and $11(26.8 \%)$ had a MR done with 0,1 and 2 sources, respectively. Finally, among the 56 patients without RF: $6(10.7 \%), 47(83.9 \%), 3(5.4 \%)$ had MR based on 0,1 and 2 sources, respectively. Nevertheless, among the 34 MR performed without the patient interview, only 10 cases were justified by a former history of dementia or auditory troubles. Moreover, 2 errors with a clinical outcome were identified in a MR during this study. They occurred on patients with more than $1 \mathrm{RF}$ and less than 2 relevant sources.

Conclusions: Expected scores of MR completed were not fully achieved at 24 and $72 \mathrm{~h}$ after admission. Only $20.6 \%$ were correctly performed with at least 2 relevant information sources. If we consider that a patient with no RF and only 1 relevant source is acceptable: this percentage could reach $57.9 \%$. This study highlights the need of relevant sources to perform a reliable MR. We stated that every MR must include at least the patient interview and another relevant source if the patient have a $R F \geq 1$. A distance learning educational program currently under development is warranted to further improve the efficiency of MR performed in surgery's wards.

Disclosure of Interest: None Declared. 


\section{Hospital pharmacy: clinical education}

\section{HP-CE05}

Oral medication dispensed in clinical trials in oncology

T. Calleja Chuclá ${ }^{1, *}$, F. Busto Fernández ${ }^{1}$, M. Calvin Lamas ${ }^{1}$, C. Martínez Roca ${ }^{1}$, E. Fernández Gabriel ${ }^{1}$, I. Martín Herranz ${ }^{1}$

${ }^{1}$ Pharmacy, Complejo Hospitalario Universitario De A Coruña, A Coruña, Spain

Background and Objective: To describe the evolution in clinical trials (CT) in oncology with medicine in solid oral pharmaceutical forms dispensed by the pharmacy service (PS) and its impact on the outpatient pharmacy service.

Setting and Method: Retrospective observational study. Study period of 3 years (2010-2012), carried out at a tertiary university hospital complex with 1,419 beds. Data source: CT management programme $\left(\operatorname{gec} \cos ^{\circledR}\right)$, reports and panel controlling the activity of the pharmacy service. We quantified the $\mathrm{CT}$ for oncology in which medicines in solid oral pharmaceutical forms were studied, the number of patients included, and the number of dispensations. A review was made of the number of $\mathrm{CT}$ in which the promoter provided an information sheet on the medicine, with regard to the guidelines for its administration and conservation.

Results: During the study period, a total of 176 CT were active in 2010, 220 in 2011 and 225 in 2012, with 77, 82 and 80 corresponding to oncology respectively. Out of the CT for oncology, 24 in 2010, 36 in 2011 and 35 in 2012 studied medicines administered orally (an increase of $12.6 \%$ between 2010 and 2012). Dispensing according to pathologies in 2012 was $10 \mathrm{CT}$ in breast cancer (everolimus, dasatinib, letrozole, anastrozole, motesanib, exemestane, sunitinib, capecitabine, sorafenib), 9 CT for non-microcytic lung cancer (inaparib, AZD4547, erlotinib, crizotinib, tivantinib, afatinib and olaparib), 5 CT for colorectal cancer (capecitabine, linifanib, axitinib and nintedanib), 2 CT for renal cancer (dovitinib, sunitinib), 2 CT for prostate cancer (abiraterone, cabozantinib), $1 \mathrm{CT}$ for glioblastoma (dacotinib), $1 \mathrm{CT}$ for hepatocellular carcinoma (linifanib), $1 \mathrm{CT}$ for melanoma (vemurafenib), $1 \mathrm{CT}$ for thyroid cancer (lenvatinib) and $1 \mathrm{CT}$ for neuroendocrine tumour (axitinib) and $1 \mathrm{CT}$ for GIST (imatinib). The number of patients and dispensations with oral medicine per year was: in 2010: 17 patients and 87 dispensations, in 2011: 29 patients and 153 dispensations and in 2012: 37 patients and 212 dispensations (an increase between 2010 and 2012 of $58 \%$ in terms of the number of patients, and $143 \%$ in terms of the number of dispensations).

The promoters did not provide information sheets in any of the clinical trials for the oral medicines to inform patients about taking the medicine according to the requirements of the protocol.

Conclusions: The Pharmacy Service plays a very important role in the performance of clinical trials, by dispensing oral medicines to patients in the outpatient oncological consultancy, providing them with information and indicating how they should correctly take the medicine involved in the clinical trial. The increase in the number of oral doses results in an increase in the consultation time dedicated to the oncological outpatient. There are serious deficiencies on the part of promoters in providing information in written format on the correct administration of the oral pharmacotherapy that meets the requirements of the clinical research protocol.

Disclosure of Interest: None Declared.

\section{HP-CE06}

Medication reconciliation in GZA hospitals

\author{
A. Djemie ${ }^{1, *}$, F. Declau ${ }^{1}$ \\ ${ }^{1}$ GZA Hospitals, Antwerp, Belgium
}

Background and Objective: Medication errors are the most common type of errors that affect the safety of the patient. They can occur during every phase of the hospitalization (admission, transfer, discharge) and at any point in the medication use (ordering, transcribing, dispensing, administrating, and monitoring). It is important that these errors are detected as soon as possible for the benefit of the patient. Medication reconciliation is thus an important factor in reducing medication errors. In this paper we investigated the current method of medication reconciliation upon admission in GZA hospitals (Belgium).

Setting and Method: Every patient who met the inclusion criteria received a blank medication scheme. The patient was encouraged to participate with his/her general practitioner or pharmacist in order to fill out the preadmission medication list. Upon admission the medication information was reviewed and also compared with the medication orders finally imported in the electronic prescription program. Errors on the medication list and discrepancies during transcription were registered and categorized according to the NCC MERP taxonomy.

Results: Of the 184 patients who received a blank medication scheme, 168 were admitted in the hospital. Of this group 114 medication schemes were received. Concerning the errors made, 95 were registered on the preadmission medication lists and 123 during transcription, respectively $14.6 \%$ and $18.9 \%$ of all the medications listed. The preadmission medication lists possessed only category A errors and no pADEs. Upon transcription, errors of category A, B and $\mathrm{C}$ were recovered. No statistical difference was found between the medications lists filled in by the patient himself or a healthcare provider such as a pharmacist or physician.

Conclusions: The key to reduce medication errors at admission is the acquisition of the best possible medication history. This can be achieved by interviewing the patient in a systematic way. Healthcare providers and patients should be encouraged to recover the best possible medication history. Once this information is obtained, it has to be verified and reconciled with at least one other reliable source such as a pharmacist, physician, nurse or a family member.

Disclosure of Interest: None Declared.

\section{HP-CE07}

Implementation of quality prescribing indicators and compliance by an Internal Medicine Team.

\section{E. Campos-Davila ${ }^{1, *}$, G. Perez-Vazquez ${ }^{2}$, J. J. Ramos-Baez}

${ }^{1}$ Pharmacy, ${ }^{2}$ Internal Medicine, Hospital SAS La Línea, La Linea de La Concepcion, Spain

Background and Objective: To describe the implementation methodology of three quality prescribing indicators in a medical team and measure their compliance after a year.

Setting and Method: The Andalusian Public Health Care Service have developed some indicators based on the selection of drugs which support better evidence of efficiency within several therapeutic groups on areas of prescribing where more deviations were detected in the past. This is a retrospective observational study that measures the prescription percentage of three of these indicators before and after an educational program consisting of clinical sessions, meetings with the head of the medical team and/or meetings with medical direction and management direction. Doctors also had information on their results every three months. The prescription rates were measured in November 2011, before the beginning of the educational program, and in October 2012, when it had ended. Prescribing data were obtained from the computerized pharmacy records of reimbursed drugs Program (Microstrategy ${ }^{\circledR}$ ) of all members who belonged to the medical team at least one month during the time studied. 
Main outcome measures: Percentage of omeprazole prescribed versus total of proton pump inhibitors, percentage of simvastatin prescribed versus total of hypolipemiants and percentage of ACEI versus total of renin-angiotensin-aldosterone-system (RAAS) inhibitors.

Results: The initial team of eight doctors was monitored. At the end of the study only one had left and no one new was incorporated. Three clinical sessions were given by the pharmacist in charge, plus two meetings between him and the chief doctor, and another two between the pharmacist, Chief Doctor and Medical Director. Omeprazole prescription was $63.93 \%$ at the beginning and increased to $82.68 \%$ a year later. Simvastatin prescribing also increased from $27.63 \%$ to $43.8 \%$, and the percentage of ACEI rose from $27.34 \%$ to $55.02 \%$ at the end of the study.

Conclusions: The educational prescribing program, based on clinical evidence guidelines, had good acceptance between the clinicians and that led to better prescribing practices.

Disclosure of Interest: None Declared.

\section{HP-CE08}

Interests of a pharmaceutical antenna within care units in psychiatric hospital: demonstration by Archimed

\section{A. Razurel ${ }^{1, *}$, M. Gaudoneix-Taieb ${ }^{1}$, C. Houssou ${ }^{2}$, P. Beauverie ${ }^{1}$ ${ }^{1}$ Pharmacy, ${ }^{2}$ Psychiatric unit, GH Paul Guiraud, Villejuif, France}

Background and Objective: The pharmacy in Paul Guiraud psychiatric hospital has two distinct entities: a central pharmacy at Villejuif and a pharmaceutical antenna at Clamart. According to an order of April 2011, "hospitals have to implement studies on risks, a priori and a posteriori, incurred by patients and related to medicinal therapy". To answer these requirements, we have realized risk cartography a priori and have compared results of two kinds of care units (CU): CU with contract including risk management, pharmaceutical antenna and pharmacist as close as possible to medical team versus CU with no contract, no pharmaceutical antenna and no pharmacist close to medical team.

Setting and Method: Two different hospital structures: (1) Villejuif, monastic architecture with a central pharmacy and no daily pharmaceutical action in CU and (2) Clamart, modern architecture with a pharmaceutical antenna and daily pharmaceutical actions in CU. They were randomized: 4CU/30 (1) and 2CU/6 (2). Risk cartography was conducted using Archimed, an Excel ${ }^{\circledR}$ tool with a 4 chapters questionnaire (I, II, III, IV) developed by Observatory for drugs, medical devices and therapeutic innovations (OMEDIT). Audits were run by pharmacist or resident pharmacist and senior nurse or senior doctor in charge of risk management. Audits were addressed to nurse, CU's senior nurse and CU's senior psychiatrist.

Main outcome measures: There were 166 questions with binary answer. Every question corresponded to a potential identified risk and was connected to the process to medicinal therapy. Questionnaries were treated by informatic tests and results expressed in percentage of risk. Results: The global risk in Clamart is lower than that in Villejuif (38 \%/45 \%). I. Structural risk: a) organization $63 \%$ (1)/59\% (2) and b) Management $79 \%$ (1)/84 \% (2). II. Politics of medicine's security: a) prevention $55 \%(1) / 47 \%$ (2) and b) piloting $77 \%(1) /$ $35 \%$ (2). III. Security therapeutic management: a) input/output of patients $21 \%(1) / 29 \%(2)$, b) prescription/dispensation $35 \%$ (1)/ $30 \%$ (2) and c) preparation/administration $23 \%$ in (1 and 2). IV. Security storage: $31 \%$ (1)/29\% (2).

Conclusions: Score at the axis II is significantly different $(p<0.01)$ between both sites and results are in favour of (2): knowledge and application of procedures, staff training and information, feedback experience rate, good use of medicine (specific management of "medicine at risk", drug information), synergy antenna/CU (contractualized organization, regular evaluations to $\mathrm{CU}$ by pharmacy, carriers trained in the specificity of products) are increased. Besides this, the data given by Archimed are confirmed by other approaches: professional practices evaluation... Archimed presents a sensibility to demonstrate the interest of a pharmaceutical antenna and the importance of a pharmacist as close as possible to medical team in order to improve the quality of medicinal management and support the development of the clinical pharmacy.

Disclosure of Interest: None Declared.

\section{HP-CE09}

Self-assessment of the therapeutic education program in chronic heart failure

\section{S. Guirao ${ }^{1, *}$, L. Eliahou ${ }^{2}$, B. Leroy ${ }^{2}$, A. Charlier ${ }^{2}$,} M.-C. Chaumais ${ }^{1}$

${ }^{1}$ Pharmacy department, ${ }^{2}$ Cardiology department, Antoine Béclère Hospital, Clamart, France

Background and Objective: Since 2011, the therapeutic education program in chronic heart failure (CHF) entitled ETIC, is active in the cardiology department of Béclère Hospital and included until now 89 patients.

In order to improve the program regarding practices, content, organization and coordination, an annual self-assessment is requested by the Hight Health Authority (HAS) in France. The objective of this work was to perform a self-assessment based on HAS guidelines through a collective and participatory approach from all of the health professional participating to this program.

Setting and Method: A review of the program activity, process and monitoring was performed and compared with the original validated program.

Main outcome measures: The primary outcome measure is based on an approach of a self-improvement program.

Results: In 2012, 47 patients out of 52 patients expected were enrolled $(90 \%)$ whose 36 completed the program. The average number of consultations per patient was 5 [3-14]. The follow-up at 2 months was achieved in 19 patients $(53 \%)$. The total number of consultations in the ETIC program were 218, of which 119 (55\%), 41 (19\%), $31(14 \%)$ and $27(12 \%)$ by the nurse, physician, dietician and pharmacist respectively.

Inclusions were systematically directly ordered by the physician or after suggestion from an ETIC team member as it were originally described. However, proceeding of the several program consultations did not systematically match the education pathway originally set leading to patient's confusion. The follow-up at 2 months was mainly performed by the referring physician of the program in a dedicated consultation. However, some patients were not available for this consultation and the follow-up was performed through a telephonic interview by the referring nurse. Regarding educational record of the patient, this tool was confirmed to be useful and adapted. Finally, this self-assessment led to increase communication and cohesion in the team.

Conclusions: As required by HAS, self-assessment of education program processes is of major importance. It relies on a quality management approach to identify with the educational team members strengths and weakness of the program. The main dysfunctions identified were a lack of communication within cardiology staff member and a different perception of the educational pathway by the team. Awareness of ETIC to the cardiology department in the next weeks as well as another program evaluation before 2014 are planned. Disclosure of Interest: None Declared. 


\section{HP-CE10}

Patient knowledge of new oral anticoagulation therapy in hip and knee arthroplasty

\section{Lejard ${ }^{1}$, J. Druon ${ }^{2}$, J. Brilhault ${ }^{2}$, X. Pourratt ${ }^{1, *}$}

${ }^{1}$ Pharmacy, ${ }^{2}$ orthopaedic surgery unit, CHU de Tours, Tours, France

Background and Objective: New oral anticoagulations (NOAC) [Dabigatran -DN and Rivaroxaban - $\mathrm{RN}$ ] have been approved for thromboprophylaxis in patients undergoing total hip (THA) or knee arthroplasty (TKA). These oral medication have been available in our hospital formulary for 3 years. Our objective was to assess patient's knowledge of anticoagulation among patients undergoing THA or TKA that were given NOAC prophylaxis.

Setting and Method: one year prospective questionnaire-based crosssectional study conducted in a 79 beds orthopaedic surgery unit.

Adult patients able to answer a questionnaire were interviewed by a pharmacist resident during their stay after surgery. Results were compared to age ( $<>70$ years), sex and arthroplasty location. Results were compared using $\mathrm{X}^{2}$ test analysis.

Main outcome measures: Knowledge of anticoagulation therapy (brand-name, therapeutic duration and administration route), drug side effects identification and their management as well as the need of information in the patient point of view.

Results: 52 patients $[\mathrm{M} / \mathrm{F}=27 / 25 ; \mathrm{RN}$ : $46, \mathrm{BN}$ : 6] corresponding to $42 \%$ of eligible population; primary THA $=32$, primary TKA $=16$ and revision $\mathrm{THA}=4$, mean age $=65.9(34-88)$ and ere interviewed over one year.

Sixty-four percent of the patients knew that they received an anticoagulation prophylaxis. Among them $90 \%$ knew the route, $24 \%$ the average duration treatment and $12 \%$ the brand-name. About $35 \%$ of patients were able to identify the side effects and the necessity to refer to a physician if occurred. Knowledge were higher in female, TKA patients and patients under 70 years old $[p<0.05]$. Conclusions: This preliminary study showed the bad patient's knowledge of NOAC and the problems that may occur with the use expansion in cardiology indications. Patients need more information; in the study $71 \%$ of them were applicant for it. In fact the clinical pharmacist should, during drug reconciliation procedure at discharge, approach the problem and verify the understanding with the patient. Disclosure of Interest: None Declared.

\section{HP-CE11}

Lifestyles in Type 2 Diabetic patients treated with insulin

\section{Caramona ${ }^{1, *}$, M. C. Patrão ${ }^{1,2}$, M. Carvalheiro ${ }^{3}$, J. Relvas ${ }^{4,5}$}

${ }^{1}$ Pharmacology and Pharmaceutical Care Group, Faculty of Pharmacy, Coimbra, ${ }^{2}$ Coimbra University, Faculty of Pharmcy, coimbra, ${ }^{3}$ Endocrinology, Diabetes \& Metabolism, ${ }^{4}$ Psiquiatric, ${ }^{5}$ Psiquiatric and Health Services, University Hospital, Coimbra, Portugal

Background and Objective: The type 2 diabetes in accordance with epidemiological studies, has been assigned to the western lifestyle, characterized by an increase in obesity, sedentary lifestyle and an energy diet rich. This study aims to assess the difficulties of adherence to diet, exercise, insulin taken and self-monitoring of blood glucose in type 2 diabetic patients treated with insulin.

Setting and Method: Was developed at the Department of Endocrinology, Diabetes and Metabolism, from Coimbra University Hospital. A convenience sample, with $\mathrm{N}=63$ patients $(57.3 \%$ of the population) following the inclusion criteria: age $20-80$, diagnosed disease at least $1 \mathrm{~A}$, the diagnosis criteria of the International Diabetes
Federation and International Society, using outpatient, with absence of pregnancy, without acute illness or depression, and normal intellectual development and with informed consent for participation. The attribute variables were gender, age, diagnosis age, $\mathrm{HbAlc}$, fasting glucose and BMI, concomitant diseases/risk factors, macrovascular and chronic complications. The data were collected during the months April-May 2011, with specific semistructured interview. They were entered in SPSS version 17.0. The quantitative analysis was descriptive in a cross-sectional study.

Main outcome measures: The diagnosis age, $\mathrm{HbAlc}$, fasting glucose and BMI, concomitant diseases/risk factors, macrovascular and chronic complications were the basis for the purpose hypotheses (HI). $\mathrm{HI} 1$ if the clinical indicators are not controlled, then the difficulties in adherence behavior are high. HI2 the age and diagnosis age increases, then the behavioral problems with compliance will be high.

Results: The data show that the difficulties of adherence to diet $(93.7 \%)$, exercise $(95.2 \%)$ and self-monitoring blood glucose $(38.1 \%)$ are very high. The glycated hemoglobin (HbA1c) with mean (M) $8.8 \%$ and Standard Deviation (SD) $1.7 \%$, Body Mass Index (BMI), (M) 29.2 (SD) 5.4, fasting blood glucose (M) $160.2 \mathrm{mg} / \mathrm{dl}$ (SD) 60.8 are not controlled. There was an association between HbA1c and difficulty in self-monitoring blood glucose $(p<0.05)$. BMI, Age and Diagnostic age are associated with adherence difficulties to diet, exercise and self-monitoring $(p<0.05)$.

Conclusions: The patients don't have healthy lifestyles. They are poorly informed about this silent disease that they have for many years.

Once installed, the diabetes is difficult to control. It arises therefore a need to develop strategies to prevent this kind of disease. The pharmacist through their abilities and skills physical information and guide the patients.

Disclosure of Interest: None Declared.

\section{HP-CE12}

Implementing a Clinical Pharmacy Practice Service in South East Norway

E. Trapnes ${ }^{1, *}$, J. B. Foss ${ }^{1}$, M. Lea ${ }^{1}$, H. Steen ${ }^{1}$, M. Davidsson ${ }^{1}$, L. Mathiesen ${ }^{1}$

${ }^{1}$ South-East Norway Pharmaceutical Trust, Oslo, Norway

Background and Objective: Although clinical pharmacy services are scarce in Norway, demand in hospitals is growing. In 2012, a national agreement among hospital pharmacies was reached on implementing the method of Integrated Medicines Management (IMM). This presentation focus on how South-East Norway Pharmaceutical Trust have been working for the past $1 \frac{1}{2}$ year to increase competence in 15 different hospital pharmacies in the region and describes strategies to implement IMM in hospitals. Our achievements, together with an analysis of probable important factors for success are presented.

Results:

Program Description

- Three experienced clinical pharmacists were trained in the (L)IMM model at the Central Norway Pharmaceutical Trust and by peer-learning locally. They developed a course in IMM for clinical pharmacists in the region.

- The course consisted of 3 days of theory including case training, and 8 days of clinical practice in medicines reconciliation and medicines review under supervision.

- Five additional supervisors spread around the region were trained

- Independent work by the attendants performed following the supervision period was thoroughly reviewed and given feedback 
- Completed program leads to certification and strategies to maintain quality and future loyalty to the model are currently under discussion

Parallell strategic work:

- Presenting and marketing in a diversity of foras including hospital management and medicine advisory boards

- Research projects and increasing clinical pharmacy competence:

- 23 masters in clinical pharmacy completed by 2015

- 7 master thesis on medicines reconciliation

- 2 research projects on information of drug use on discharge

- IMM PhD project started

- National collaboration among Hospital Pharmacy Trusts

Conclusions: By the end of 2013, 35 clinical pharmacists have attended the program, and hospitals have begun requesting our services. We believe that organizing a project with a separate budget and strong leadership involvement has contributed to our achievements. A national agreement on IMM and the national patient safety program on medicines reconciliation were also important contributors. Disclosure of Interest: None Declared.

\section{HP-CE13}

Self-efficacy evaluation in type 2 diabetic patients treated with insulin

\section{Caramona ${ }^{1, *}$, M. C. Patrão ${ }^{1,2}$, M. Carvalheiro ${ }^{3}$, J. Relvas ${ }^{4,5}$}

${ }^{1}$ Pharmacology and Pharmaceutical Care Group, Faculty of Pharmacy, Coimbra, ${ }^{2}$ Coimbra University, Faculty of Pharmcy, coimbra, ${ }^{3}$ Endocrinology, Diabetes \& Metabolism, ${ }^{4}$ Psiquiatric, ${ }^{5}$ Psiquiatric and Health Services, University Hospital, Coimbra, Portugal

Background and Objective: All the health professionals dedicated to solve patients problems, feel grow a platoon of endless type 2 diabetics. This study aims to assess self-efficacy, a variable derived from the theory of Banduras'learning, as a predictor of adherence behaviors to health in type 2 diabetic patients treated with insulin.

Setting and Method: The study was developed in the Department of Endocrinology, Diabetes and Metabolism Coimbra University Hospital. A convenience sample, with $\mathrm{N}=63$ patients $(57.3 \%$ of the population) and the inclusion criteria were :age 20-80, diagnosed disease at least $1 \mathrm{~A}$, criteria of diagnosis diabetes the International Society/International Diabetes Federation, outpatients, absence of pregnancy, acute illness or without depression, normal intellectual development and consent informed participation. The attribute variables were gender, age, diagnosis age, educational level, Body Mass Index (BMI), fasting blood glucose. The data collection during the months April-May 2011, with the Self-Efficacy Scale for Diabetes (SEDS), adapted to the Portuguese population and subscales of content analysis: healthcare(SED-M), diabetes(SED-D), general situations (SED-G). We used SPSS version 17.0. The quantitative analysis was descriptive and cross-sectional study.

Main outcome measures: The research hypotheses were (HI): HI1 if BMI and fasting blood glucose are not controlled, then the self-efficacy is low; HI2 the age and age at diagnosis are advanced, soefficacy decreases. HI3 if academic level increases, then the levelefficacy is increased.

Results: The findings were: BMI Medium (M) 29.2, standard deviation (SD) 5.4, fasting blood glucose (M) $160.2 \mathrm{mg} / \mathrm{dl}$ (SD) 60.8, are not being controlled. In the global-efficacy (SEDS), (M) 8.8 and (SD) 3.6. Subscales SED-M (M) 9.5, (SD) 3.1; SED-D and SED-G (M) 9.1, (SD) 3.7. There was an association of SEDS, SED-D, SED-G and BMI $(p<0.05)$; SEDS, SED-D, SED-G and fasting blood glucose $(p<0.05)$. Age and level of educational attainment are associated with SEDS and all subscales $(p<.05)$. Age at diagnosis associated with SEDS, SED-D SED and SED-G $(p<0.05)$.

Conclusions: The Programme for Prevention and Control of Diabetes, to be fulfilled, must be supported by a structure where the model of care has the patient as central and not the disease. Primary prevention will be necessarily a continuous solution and present all the time for all the patients. With our experience the pharmacist must be envolved on it!

Disclosure of Interest: None Declared.

\section{Therapeutic drug monitoring and pharmacokinetics}

\section{TDMP11}

Does administration of gentamicin require management of a clinical pharmacist?

M. Goboova ${ }^{1, *}$, V. Kissova ${ }^{1}$, L. Salkovska ${ }^{2}$, V. Kakosova ${ }^{3}$, M. Kuzelova ${ }^{4}$

${ }^{1}$ Department of Internal Medicine and Clinical Pharmacology, ${ }^{2}$ Department of Laboratory Medicine, Teaching Hospital, Nitra, ${ }^{3}$ Department of Pharmacy, University Hospital, ${ }^{4}$ Department of Pharmacology and Toxicology, Faculty of Pharmacy, Comenius University, Bratislava, Slovakia

Background and Objective: Concern about nephrotoxicity has often led to reluctance to prescribe higher appropriate doses of gentamicin relative to pharmacokinetic parameters. Patients often receive insufficient dosage in clinical practice. The aim of the study was to confirm the need of management of a clinical pharmacist in determining optimal doses for the patients.

Setting and Method: Retrospective study includes 221 (175 men, 46 women) patients hospitalized at the Teaching Hospital Nitra and treated by gentamicin during three years (June 2010-June 2013). The adjustment of doses was provided by the clinical pharmacist before TDM or after TDM. Gentamicin concentrations were measured by the FPIA (Fluorescence Polarization Immunoassay) method.

Main outcome measures: Number of patients with optimal levels of gentamicin in group with the adjustment of dose before TDM and in group with the adjustment of dose after TDM.

Results: The patients were divided into 2 groups. The first group included 44 (the average age $56 \pm 17$ years) patients, whose dose had been adjusted by the clinical pharmacist before TDM according to pharmacokinetic parameters at the request of doctors. The second group included 177 (the average age $56 \pm 18$ years) patients, whose dose had been adjusted by the clinical pharmacist after TDM. 42 (95\%) patients in the first group had optimal both levels, 1 patient had high trough level and 1 patient had low peak level. Only 22 $\left(13 \% ; 61 \pm 13\right.$ years; $\left.\mathrm{ClCr}_{\mathrm{CG}} \mathrm{ABW} 94.3 \pm 42.9 \mathrm{ml} / \mathrm{min} / 1.73 \mathrm{~m}^{2}\right)$ patients in the second group had both levels optimal, 137 (77\%; $53 \pm 17$ years; $\mathrm{ClCr}_{\mathrm{CG}} \mathrm{ABW} 103.2 \pm 40.2 \mathrm{ml} / \mathrm{min} / 1.73 \mathrm{~m}^{2}$ ) patients had low peak levels and $18\left(10 \% ; 73 \pm 11\right.$ years; $\mathrm{ClCr}_{\mathrm{CG}} \mathrm{ABW}$ $65.4 \pm 24.8 \mathrm{ml} / \mathrm{min} / 1.73 \mathrm{~m}^{2}$ ) had high trough levels. The clinical pharmacist adjusted the dose for this group according to TDM and pharmacokinetic parameters. The results after adjustment of the dose were much better. 146 patients $(82 \%)$ had both levels optimal and 31 $\left(18 \% ; 54 \pm 17\right.$ years; $\left.\mathrm{ClCr}_{\mathrm{CG}} \mathrm{ABW} 109.6 \pm 47.3 \mathrm{ml} / \mathrm{min} / 1.73 \mathrm{~m}^{2}\right)$ patients had low peak levels.

Conclusions: Our results highlighted a problem of administration of gentamicin. The clinical pharmacist has to adjust the dosage regimens according to pharmacokinetic parameters and TDM. The clinical effect of gentamicin would not be reached without the pharmaceutical care and intervention was accepted by doctors.

Disclosure of Interest: None Declared. 


\section{TDMP12}

Are telaprevir plasma concentrations predictive of virological response?

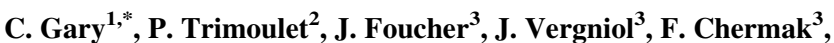
S. Bouchet ${ }^{4}$, W. Merrouche ${ }^{3}$, D. Breilh ${ }^{1}$, V. De Ledinghen ${ }^{3}$

${ }^{1}$ Pharmacokinetics, ${ }^{2}$ Virology, ${ }^{3}$ Hepatology, ${ }^{4}$ Pharmacovigilance, CHU Bordeaux, Bordeaux, France

Background and Objective: Telaprevir (TVR) is a protease inhibitor used for treatment of genotype 1 hepatitis $\mathrm{C}$ virus (HVC) infection. However, the impact of TVR pharmacokinetics (PK) on sustained virological response (SVR) has not been investigated. Our aim was to assess the relationship between TVR PK and negative HCV-RNA at W48, and SVR 6 months after the end of treatment (W72).

Setting and Method: We conducted a single-center prospective study with 42 monoinfected patients with $\mathrm{HCV}$ genotype 1 with severe fibrosis or cirrhosis, who failed to a previous treatment with pegylated interferon (PEG) and ribavirin (RIBA) treated with PEG-RIBA-TVR for 12 weeks and PEG-RIBA for 36 weeks. Trough plasma TVR concentrations (TVRc) were measured at $\mathrm{W} 1$ and $\mathrm{W} 4$. The PK/SVR relationship was performed by Mann-Whitney test. SVR was defined as an undetectable HCV RNA 6 months after stopping treatment (W72).

Results: The median [interquartile range] TVRc at W1 and W4, were $3,979[3,355,5,318] \mathrm{ng} / \mathrm{mL}$ and $4,391[3,231,5,993] \mathrm{ng} / \mathrm{mL}$, respectively. There was a significant correlation between TVRc at W1 and W4 (Spearman test, $\rho=0.68, p<0.05$ ) and a wide interindividual PK variability (coefficient of variation $44 \%$ ). Thirty-three patients achieved W48, 25 of them (76\%) had negative HCV-RNA at W48. Reasons stopping treatment were: escape (2), partial response (1), intercurrent infection (2), intolerance (2), other (1). At W48, there was a significant difference on TVRc between patients with negative HCVRNA and those with positive HCV RNA: 4,656 [3,771, 5,688] ng/mL $(\mathrm{n}=25)$ and $2,790[1,630,3,657] \mathrm{ng} / \mathrm{mL}(\mathrm{n}=8), p=0.00496$, respectively. To date, 13 patients were assessed at W72, 12 patients achieved SVR, with a median of TVRc 5,597 [4,167, 6,200] ng/mL. The patient relapsing at W52 had a TVRc of $2,991 \mathrm{ng} / \mathrm{mL}$.

Conclusions: TVRc were significantly higher in sustained virological responders at W48 comparing with nonresponders patients, suggesting a therapeutic plasma threshold for TVR.

Disclosure of Interest: None Declared.

\section{TDMP14}

Benefits of therapeutic drug monitoring of intravenous busulfan in paediatric

N. Solée ${ }^{1, *}$, P. Riera ${ }^{1}$, E. Fernández de Gamarra ${ }^{1}$, I. Badell ${ }^{2}$, M. Torrent ${ }^{2}$, M. A. Mangues ${ }^{1}$

${ }^{1}$ Pharmacy, ${ }^{2}$ Hospital de la Santa Creu i Sant Pau, Barcelona, Spain

Background and Objective: Busulfan is commonly used in preparative regimens for hematopoietic stem-cell transplantation in adults and children for a variety of malignant and non-malignant diseases. It is a drug with a small therapeutic range and a large pharmacokinetic variability that could have consequences in treatment outcomes and toxicity. It is available for oral and intravenous administration, with more and less variability respectively. The aim of this study is to assess the benefits of therapeutic drug monitoring (TDM) to guide intravenous busulfan dosing.

Setting and Method: This is a retrospective study conducted in a tertiary paediatric unit from March 2010 to June 2013. We included children treated with intravenous busulfan who underwent TDM. We applied a non-linear regression method and used ID3 software for pharmacokinetic studies. The dosing regimen aimed to reach a cumulative target exposure defined as area under the curve (AUC) of $55,000-95,000 \mathrm{ng} / \mathrm{ml} . \mathrm{h}$, depending on the conditioning protocol and based on the current literature of optimal exposures of busulfan in children.

Main outcome measures: Busulfan serum concentrations, pharmacokinetic parameters: volume of distribution $(\mathrm{Vd})$, elimination rate constant (Kel), AUC.

Results: Thirteen children with ages ranging from six months to eleven years were included in the study. The distribution of baseline diseases was: $3 / 13$ malignant and 10/13 non-malignant. Regarding the conditioning regimen, 9/13 were myeloablative and 4/13 non-myeloablative. Busulfan initial doses ranged form 2.8 and $5.2 \mathrm{mg} / \mathrm{kg} /$ day, according to the protocol and the weight band. All the patients received seizures prophylaxis with phenytoin. Median estimated Vd was $0.73 \mathrm{~L} / \mathrm{kg}(0.4-2.69 \mathrm{~L} / \mathrm{kg})$ and median estimated Kel was $0.39 \mathrm{~h}^{-1}$ $\left(0.21-0.68 \mathrm{~h}^{-1}\right)$. Twelve patients needed a dose adjustment (9 reductions and 3 increases) to guarantee a drug exposure within the desired range. Four patients had an initial estimated AUC $>28,800 \mathrm{ng} / \mathrm{ml} . \mathrm{h}$ for the first day, which might be related with a higher risk of toxicity. Three of these patients had started the treatment with a busulfan dose $>5 \mathrm{mg} / \mathrm{kg} / \mathrm{day}$. None of the 13 patients had any signs of toxicity.

Conclusions: TDM of IV busulfan in paediatric patients, in spite of its theoretical advantage of less variability compared to the oral form, is a valuable tool to guide the drug dosing.

Disclosure of Interest: None Declared.

\section{TDMP15}

Population pharmacokinetics of amikacin in non-critical care pediatric Kuwaiti patients without cystic fibrosis

\section{Y. Allanqawi ${ }^{1, *}$, E. Buhamrah ${ }^{2}$, Z. Demerdash ${ }^{3}$, J. Al-Momen ${ }^{3}$}

${ }^{1}$ Department of Pharmacy, Drug and Poison Information Unit, Al-Amiri Hospital, Ministry of Health, Kuwait, ${ }^{2}$ Pediatric Department, Al-Amiri Hospital, Ministry of Health, Kuwait, ${ }^{3}$ Pediatric Department, Al-Amiri Hospital, Ministry of Health, Kuwait, Kuwait

Background and Objective: Amikacin (AMK) is commonly used to treat gram negative infections in pediatric patients. The AMK pharmacokinetic parameters such as clearance $(\mathrm{Cl})$ and volume of distribution (Vd) displays an extensive interindividual variability's which makes it difficult to achieve safe and effective treatment. Covariates such as Age, body weight (bw), sex and creatinine clearance account for the interindividual variability in $\mathrm{Vd}$ and $\mathrm{Cl}$. Published data on AMK pharmacokinetics for Arab pediatric patients are very limited and in Kuwait are none. The aim of this study was to: (1) calculate the $\mathrm{Vd}$ and $\mathrm{Cl}$ in pediatric Kuwaiti patients without cystic fibrosis; (2) investigate the effect the covariates on the individual $\mathrm{Vd}$ and $\mathrm{Cl}$.

Setting and Method: 62 non-critical care pediatric patients older than 0.25 years of age (ranged from 0.25 to 16 years) without cystic fibrosis (29 male and 33 female) were identified for whom a confirmed steady-state AMK peak and trough levels were available. They received a multiple daily dosing regimen of AMK (4 to $15 \mathrm{mg} / \mathrm{kg}$ intravenously during $0.1 \mathrm{~h}$, two to three times per day) between 2006 and 2009. The data were collected from pediatric wards at Al-Amiri hospital-Kuwait. Assuming one compartment linear model, the Vd and $\mathrm{Cl}$ were calculated using the method of Sawchuck and Zaske. Regression analysis was performed to asses the effect of age, bw, sex and creatinine clearance on $\mathrm{Vd}$ and $\mathrm{Cl}$. 
Main outcome measures: The AMK kinetic parameters $\mathrm{Cl}$ and $\mathrm{Vd}$ were estimated for pediatric patients.

Results: The Mean $+\mathrm{Sd}$ (median, range) of the $\mathrm{Cl}$ and $\mathrm{Vd}$ were $1.77 \pm 1.38(1.46,0.19-5.03 \mathrm{l} / \mathrm{h})$ and $6.52 \pm 5.67(4.64,0.73-21.07 \mathrm{l})$ respectively. The age and bw were found to have significant effects on both $\mathrm{Cl}$ and $\mathrm{Vd}$ as indicated by the following equations: $\mathrm{Cl}=$ $0.431+0.128 \times$ age $+0.03 \times w t, \quad\left(R^{2}=0.826\right) ; \quad V d=0.892+$ $0.488 \times$ Age $+0.142 \times$ bw, $\left(R^{2}=0.86\right)$.

Conclusions: Considerable interindividual variability's in CL and V were found within the pediatric population studied. Both CL and V were increased with increasing age and wt, therefore, initial dose regimen based on age and bw models is recommended.

Disclosure of Interest: None Declared.

\section{TDMP16}

\section{Antidepressant therapy and TDM}

\section{Z. Paluch ${ }^{1, *}$, T. Richter ${ }^{2}$, L. Sadílková ${ }^{3}$, I. Vyhlídalová ${ }^{4}$, Š. Alušík ${ }^{5}$.}

${ }^{1}$ St. Elizabeth University of Health and Social Work, Bratislava, Slovakia, St. J. N. Neumann Institute, Př́bram, ${ }^{2}$ Department of Geriatrics, First Faculty of medicine, Charles University and General Faculty Hospital, Praha, ${ }^{3}$ Clinical pharmacology, Thomayer hospital, Praha, ${ }^{4}$ Clinical pharmacology, Thomayer hospital, Praha $4,{ }^{5}$ Institute for Postgraduate Medical Education, Praha, Czech Republic

Background and Objective: Therapeutic drug monitoring (TDM) has been recently extended to embrace the class of antidepressants. Most of the recommended levels have been set on the basis of data from clinical trials conducted in series of young patients without any associated diseases. In these patients, a significant association between the plasma levels and clinical effect has been demonstrated. Citalopram is among one of the most oft-used depressants in internal medicine. It belongs to the class of selective serotonin reuptake inhibitors. Its pharmacokinetics and pharmacodynamics in elderly and very old patients differ from those in the other age groups of patients. Setting and Method: We examined a total of 94 polymorbid patients taking citalopram at least at a dose of $20 \mathrm{mg}$ daily. In those enrolled into the study, through citalopram levels were determined by highperformance liquid chromatography (HPLC) with UV detection using a commercially available Benzodiazepines and TCA set (Chromsystem, Munich, Germany).

Clinical trials conducted in series of young patients without any associated diseases. In these patients, a significant association between the plasma levels and clinical effect has been demonstrated. Citalopram is among one of the most oft-used depressants in internal medicine. It belongs to the class of selective serotonin reuptake inhibitors. Its pharmacokinetics and pharmacodynamics in elderly and very old patients differ from those in the other age groups of patients. Main outcome measures: Our study was designed to determine the levels of citalopram in our polymorbid patients and the proportion of patients reaching the optimal therapeutic range while on the usual dosage schedule.

Results: Using the standard dosage schedule of $20 \mathrm{mg}$ citalopram, the therapeutic range was achieved in $60(62.2 \%)$ patients; of this number, optimal therapeutic range was achieved in only $13(13.8 \%)$ patients. The number of patients not achieving the therapeutic range was $30(31.9 \%)$; citalopram levels were not detected in another 4 $(4.3 \%)$ patients.

Conclusions: One in three polymorbid patients receiving low-dose citalopram does not reach the plasma levels within the therapeutic range. Our results show the issue of antidepressant therapy in polymorbid patients will require more attention in the future.

Disclosure of Interest: None Declared.

\section{Drug information}

\section{DI03}

Influence of an individualized medication plan with drug administration recommendations on patient-physician conversation at hospital discharge

A. F. J. Send ${ }^{1,2, *}$, K. I. Foerster ${ }^{1,2}$, B. Graf ${ }^{1,2}$, M. Schwab ${ }^{3}$, A. Gauss ${ }^{4}$, G. Rudofsky ${ }^{5}$, W. E. Haefeli, ${ }^{1,2}$, H. M. Seidling ${ }^{1,2}$

${ }^{1}$ Department of Clinical Pharmacology and Pharmacoepidemiology, ${ }^{2}$ Cooperation Unit Clinical Pharmacy, ${ }^{3}$ Department of General Internal Medicine and Psychosomatics, ${ }^{4}$ Department of Gastroenterology, ${ }^{5}$ Department of Endocrinology, University of Heidelberg, Heidelberg, Germany

Background and Objective: Patient information and education on correct drug administration is mandatory to prevent medication errors. Supplementary written instructions may improve patients' recall and knowledge of their drug therapy. At discharge and other interfaces of care information exchange is often short and fragmentary due to time constraints and interruptions. We aimed to improve patient information and ultimately the patients' knowledge at discharge by providing physicians with a medication plan that is automatically generated and contains drug administration recommendations personalized to the individual patient. Setting and Method: We observed patient-physician conversations at hospital discharge on three internal medicine wards of the University Hospital Heidelberg before (phase 1) and after (phase 2) the electronic prescribing system was equipped with a personalized printable medication plan. The amount and type of provided information on drug application and the time needed for information exchange were documented. To assess patients' knowledge, three standardized questions about their pharmacotherapy were asked after the patientphysician conversation.

Main outcome measures: The study analyzes how issuing a personalized medication plan modifies the conversation between patient and physician at discharge from the hospital.

Results: In total, 85 conversations were observed (50 during phase 1, 35 in phase 2$)$. The average duration $( \pm S D)$ of the conversations in phase 1 was $5.6 \min ( \pm 3.6)$ whereof $37.4 \%( \pm 20.9)$ of the time was spent on drug information. In phase 2 , conversation duration was similar (5.8 $\pm 3.3 \mathrm{~min})$, however, the time spent on drug information increased significantly $(61.3 \pm 23.3 \% ; p<0.01)$. During phase 1 , for $75.1 \%(151 / 201)$ of the drugs at least one administration advice was given verbally, compared to $83.4 \%(156 / 187 ; p=0.04)$ in phase 2 -however $100 \%$ of drugs in phase 2 received a written administration advice $(p<0.01)$. Four times more patients $(65.7 \%)$ answered the three questions about their pharmacotherapy correctly in phase 2 as opposed to $16.7 \%$ of patients in phase $1(p<0.01)$.

Conclusions: A personalized medication plan with drug administration recommendations improves the transfer of pharmacological information at discharge and increases patients' ad-hoc knowledge about their pharmacotherapy without delaying the process.

Disclosure of Interest: None Declared.

\section{DI04}

Assessment of Knowledge, Attitude and Quality of Life in Patients Utilized Topical Corticosteroid

B. Okuyan ${ }^{1, *}$, B. Gunduz ${ }^{1}$, S. Deniz ${ }^{1}$, S. Sahiner ${ }^{1}$, M. Sancar ${ }^{1}$, F. V. Izzettin ${ }^{1}$

${ }^{1}$ Clinical Pharmacy, Marmara University- Faculty of Pharmacy, Istanbul, Turkey 
Background and Objective: The aim of the study is to evaluate the patients' attitude and knowledge on utilization of topical corticosteroid and assess quality of life of them in community pharmacy.

Setting and Method: This cross sectional study was conducted during February 01-April 30, 2012. The participants gave their consent after fully informed about the study. Patients were eligible for the study if they were utilized topical corticosteroid at least a month and were older than 18 years old.

Main outcome measures: The questionnaire was applied to patients to determine their attitude and knowledge on utilization of topical corticosteroid in community pharmacy setting. The quality of life was measured by 'Dermatology Life Quality Index (DLQI)', which proved as reliable and valid by Ozturkcan et al. (1).

Results: Of the 56 participants (mean of age: $34.88 \pm 13.96$ years old [range 19-74]; $73.2 \%$ female), $62.5 \%$ have been graduated from university. The medical problems resulted in topical corticosteroid utilization were eczema (30.4\%), psoriasis $(8.9 \%)$, dermatitis $(8.9 \%)$, and fungal infections $(7.1 \%)$. Although $30.4 \%$ of them thought topical corticosteroid treatment safely, $33.9 \%$ of them approached cautiously to treatment with topical corticosteroid. Almost one third of participants thought the long treatment period and the greater amount utilization of topical corticosteroid were more effective. When evaluating the topical corticosteroid utilization, the only $26.8 \%$ of them applied a thin film of topical corticosteroid to the affected area. According to the information that gathered from the patients; $51.8 \%$ of them did not take any information from their health care providers about how to use and apply topical corticosteroid. The overall index score of the DLQI ranged from 0-22 with a mean of $5.95 \pm 5.32$.

Conclusions: It would be important to determine patients' attitude and knowledge toward treatment with topical corticosteroids to obtain barriers of this treatment, which were resulted in poor health outcomes.

Bibliographic references: 1. Oztürkcan S, Ermertcan AT, Eser E, Sahin MT. Cross validation of the Turkish version of dermatology life quality index. Int J Dermatol. 2006;45(11): 1300-7.

Disclosure of Interest: None Declared.

\section{DI05}

Attitude and knowledge of hospitalized patients with type 2 diabetes mellitus towards disposable insulin pens utilization

B. Okuyan", ${ }^{1,}$ B. Saglam ${ }^{1}$, E. Emre ${ }^{2}$, E. Karatas-Kocberber ${ }^{1}$, H. Hucum ${ }^{1}$, R. Demirtunc ${ }^{2}$, F. V. Izzettin ${ }^{1}$, M. Sancar ${ }^{1}$

${ }^{1}$ Clinical Pharmacy, Marmara University- Faculty of Pharmacy, ${ }^{2}$ Department of Internal Medicine, Haydarpasa Numune Training \& Research Hospital, Istanbul, Turkey

Background and Objective: The aim of this study is to evaluate attitude and knowledge of hospitalized patients with type 2 diabetes mellitus towards disposable insulin pens utilization.

Setting and Method: This cross sectional study was conducted in internal medicine ward of a research and training hospital between March 01 and April 30, 2013. Patients type 2 diabetes mellitus hospitalized with any reason during the present study were eligible if they were 18 years or older, prescribed at least one disposable insulin pen for at least 4 weeks, and accepted to participate to the present study. Main outcome measures: Six components for proper administration and storage were evaluated for disposable pen utilization (1).

Results: A total of one hundred and forty six disposable insulin pen utilized by one hundred and one type 2 diabetic patients (mean of age: $63.74 \pm 1.24$ years old; male/female: $47 / 54$ ) were evaluated in this study. In total of 101 patients, $51.1 \%$ were 65 years old or older. Of all, $27.7 \%$ had no illiterate and only $19.9 \%$ of them had a high school degree. When evaluating patients' recent haemoglobin A1C level, which was gathered in eighty-six patients, twenty- nine patients' haemoglobin A1C levels were equal and less than $6.5 \%$. Patients received education regarding insulin pen utilization mostly from nurse $(56.4 \%)$ and physician $(34.7 \%)$ and only two patients gathered information about insulin pen administration from their pharmacist. Although $97 \%$ of them thought that they administered disposable insulin pen correctly, most of them were failure to discard the insulin pen before expire date reported by manufacturer $(68.1 \%)$, failure to prime needle $(65.3 \%)$, failure to store in-use pen at room temperature (58.4\%), and failure to hold needle adequate period before withdrawal of pen needle from skin $(49.5 \%)$. When evaluated both frequency of patients' insulin injection daily and patients' needle used in daily, $81.2 \%$ of them properly changed needle of insulin pen before each injection. However, when evaluated discard pen needle after each injection, $39.6 \%$ of them stored their disposable insulin pen with needle.

Conclusions: It was found that most of the key components for proper utilization of disposable insulin pen were incorrectly applied by hospitalized patients with type 2 diabetes mellitus. For proper disposable insulin pen utilization, the patient education given by pharmacist, which was found less in the present study, would be evaluated in the further study.

Bibliographic references: 1 . Mitchell VD, Porter K, Beatty SJ. Administration technique and storage of disposable insulin pens reported by patients with diabetes. Diabetes Educ. 2012, 38(5):651-8. Disclosure of Interest: None Declared.

\section{DI06}

The Determination of Knowledge and Attitude toward Contraception of Women Utilized Isotretinoin: preliminary study

B. Okuyan ${ }^{1, *}$, A. H. Ozdemir ${ }^{1}$, S. Deniz ${ }^{1}$, M. Yilmaz ${ }^{1}$, F. V. Izzettin ${ }^{1}$, M. Sancar ${ }^{1}$

${ }^{1}$ Clinical Pharmacy, Marmara University- Faculty of Pharmacy, Istanbul, Turkey

Background and Objective: The aim of this study is to determine knowledge and attitude toward contraception of women utilized isotretinoin in the community pharmacy.

Setting and Method: This cross sectional study was conducted in community pharmacies between February 01 and May 31, 2013. Demographic and clinical data were collected from women utilized isotretinoin who were over the age of 18 and accepted to participate to the present study.

Main outcome measures: Knowledge and attitude toward contraception was evaluated by the questionnaire designed by Boucher and Beaulac-Baillargeon (1).

Results: Forty-one women utilized isotretinoin (mean of age: $25.15 \pm 4.44$ years old) were conducted in this study. Only one of seven patients, who had an indication for contraception, had a prescription for contraception when starting isotretinoin therapy. All of the patients reported that they had information about necessity of contraception while using isotretinoin. However, $65.9 \%$ of them did not know necessity of using two effective contraceptive methods during treatment. When evaluating patients, who had an indication for contraception, five of them knew necessity of using two effective contraceptive methods during treatment and only 3 of them applied two effective contraceptive methods during isotretinoin treatment. Participants received information regarding necessity of contraception during utilization of isotretinoin from physician $(80.5 \%)$, drug leaflet $(46.4 \%)$, and pharmacists $(9.8 \%)$. Of all participants, $78 \%$ had knowledge about applying a pregnancy test before starting isotretinoin and $61 \%$ of them reported that they knew necessity of regularly 
applying pregnancy test during this treatment. Of all participants, $65.9 \%$ had known important of continuing effective double contraception for 1 month after the last isotretinoin dose. Six of seven patients, who had an indication for contraception, knew need of emergency contraceptive after unprotected sexual intercourse.

Conclusions: Although the participants reported that they know necessities of contraception during isotretinoin therapy, most of them had less information about contraception methods during isotretinoin therapy. The sample size is one of the limitations of the present study. The present preliminary study should be improved by increasing sample size with different methods in further study.

Bibliographic references: Boucher N, Beaulac-Baillargeon. Pregnancy prevention among women taking isotretinoin: failure to comply with the recommendations. Can Fam Physician. 2006 Mar;52:338-9. Disclosure of Interest: None Declared.

\section{DI07}

Efficacy and tolerance of topical $1 \%$ rapamycin in cutaneous tuberous sclerosis patients.

\section{J. Ott ${ }^{1, *}$, F. Gass-Jégu ${ }^{1}$, A. Gairard-Dory ${ }^{1}$, B. Gourieux ${ }^{1}$, D. Lipsker ${ }^{2}$}

${ }^{1}$ Pharmacy-Sterilization Department, ${ }^{2}$ Dermatology Departement, Strasbourg University Hospital, Strasbourg, France

Background and Objective: Tuberous sclerosis (TS) is an autosomal dominant neuro-dermatosis characterized by the development of hamartomatous tumours in multiple organs. The prevalence is estimated at one per 6,000 in the general population.

Facial angiofibromas, a cutaneous manifestation of TS, appear from three to seven years of age as innumerable pink papules that progressively enlarge and multiply over time and have been resistant to surgical treatments.

The aim of this study was to evaluate the efficacy and tolerance of topical $1 \%$ rapamycin, applied twice a day for facial angiofibromas. Setting and Method: This retrospective study included patients with cutaneous manifestations of which facial angiofibromas. They were treated with topical $1 \%$ rapamycin for 6 to 18 months according to patients. The stage of their disease did not require oral treatment with everolimus yet. A satisfaction questionnaire was performed for two patients and for two patient's parents.

Results: In the four patients treated $(25,16,12,9$ years old), an improvement of facial angiofibromas was observed 4 to 6 months after the beginning of the treatment with both flattening of the angiofibromas and reduction of erythema, and then a stabilization of the lesions. After 18 months all the lesions disappeared for the patient who used this treatment since this time. None irritation neither adverse reactions were reported by the patients with topical rapamycin. Patients found this treatment a little restrictive because cutaneous penetration time is long but they were very satisfied about the efficacy of this cream and asked to pursue their treatment.

Published case reports of topical $1 \%$ rapamycin for angiofibromas in TS reported a decrease of erythema within a week of starting the treatment, and shortly thereafter an improvement in skin texture. Another case report reported that one month and a half after starting treatment, all lesions disappeared, therefore treatment was discontinued. Conclusions: Dermatologist's experience confirm a real improvement after one year of good treatment adherence. While this treatment shows a potential to be a first-line management for facial angiofibromas in TS and appears safe to start in early childhood, large-scale clinical trials are needed to validate the safety and effectiveness of this novel treatment.

Disclosure of Interest: None Declared.

\section{DI08}

Ivacaftor in adults with cystic fibrosis: one-year experience in the real world setting

I. Trinh ${ }^{1, *}$, E. Bermudez ${ }^{1}$, N. Abbas ${ }^{1}$, F. Viguier ${ }^{1}$, D. Hubert ${ }^{2}$, C. Guérin ${ }^{1}$, F. Chast ${ }^{1}$

${ }^{1}$ Pharmacy department, ${ }^{2}$ Pulmonology department, Hôpitaux Universitaires Paris Centre, AP HP, Site Cochin, Paris, France

Background and Objective: Cystic Fibrosis (CF) is an autosomal recessive genetic disease due to mutations of the cystic fibrosis transmembrane conductance regulator (CFTR) gene. More than 1900 CFTR mutations have been described and grouped in 6 classes according to their mechanism of action on the CFTR protein.

Ivacaftor (VX-770) is a small molecule acting as a potentiator increasing the time that CFTR channels at the cell surface remain open.

Ivacaftor has been available in France since April 2012 for CF patients aged 6 or older with a G551D mutation, the most frequent of the class 3 mutations (2-5\% of patients).

Setting and Method: A retrospective study was conducted in patients treated by Ivacaftor $(150 \mathrm{mg}$ bid) in the adult CF centre of the Pulmonology department in order to analyze efficacy and safety of Ivacaftor.

Main outcome measures: Collected data included general data (birth date, gender, date of first treatment with ivacaftor) and clinical data (FEV1, sweat chloride, body weight, number of antibiotic IV courses) after 24 and 48 weeks (W24, W48) of treatment which were compared to baseline data.

Results: The survey included 7 patients (6 males and 1 female) with a mean age of 37 years [26-52]. Six patients had been treated since May 2012 and one patient since January 2011 when participating to an open-label extension clinical trial.

Mean FEV1 before treatment was $44.1 \%$ pred. [24 to 75]. There was an increase from baseline of $10.7[5 ; 22]$ and $11.0[-2 ;+26]$ percentage points of predicted FEV1, respectively at W24 and W48. Furthermore, body weight [min; max] increased of $2.2[-1.6 ;+8.7]$ and $2.6[-0.7 ;+10.0] \mathrm{kg}$, at W24 and W48; one patient discontinued continuous nocturnal enteral feeding.

The number of IV antibiotic courses decreased of $41 \%$ (3.7/year before treatment in comparison to 2.1/year after treatment), related to a significant decrease of pulmonary exacerbations.

Mean sweat chloride [min-max], a biomarker of CFTR activity, decreased from $94 \mathrm{mmol} / \mathrm{L}[62 ; 113]$ at baseline to $36 \mathrm{mmol} / \mathrm{L}[18$; 56] after treatment, showing a reduction in the sweat chloride level to values below the diagnostic threshold for $\mathrm{CF}(60 \mathrm{mmol} / \mathrm{L})$.

One patient experienced headache, dizziness and myalgias the first days of treatement, another one had asthma requiring steroids and tachyarythmia with atrial fibrillation. Nevertheless, Ivacaftor was not discontinued.

Conclusions: Our results in these 7 French CF adult patients in real world setting showed a similar benefit (increase in FEV1 and weight) and safety profile than that seen in clinical trials.

Disclosure of Interest: None Declared.

\section{DI09}

The determination of knowledge and attitude of women toward oral contraceptive pills

B. Okuyan ${ }^{1, *}$, M. Yilmaz ${ }^{1}$, S. Deniz ${ }^{1}$, A. H. Ozdemir ${ }^{1}$, M. Sancar ${ }^{1}$, F. V. Izzettin ${ }^{1}$

${ }^{1}$ Clinical Pharmacy, Marmara University- Faculty of Pharmacy, Istanbul, Turkey 
Background and Objective: The aim of this study is to evaluate knowledge and attitude toward oral contraception and determine adverse drug reactions in women utilized oral contraceptive pills in the community pharmacy setting.

Setting and Method: This cross sectional study was conducted in community pharmacies between February 01 and May 31, 2013. Demographic and clinical data were collected from the women utilized oral contraceptive pills, who were over the age of 18 and accepted to participate to the present study.

Main outcome measures: Knowledge and attitude toward oral contraceptive of women, adverse drug reactions.

Results: Eighty women utilized oral contraceptive (mean of age: $31.26 \pm 5.4$ years old) were conducted in this study. $93.8 \%$ of them were married. $22.5 \%$ of them were smoking. The commonly used oral contraceptives were drospirenone/ethinyl estradiol $(62.5 \%)$ and ethinyl estradiol/levonorgestrel (13.8\%). Of the women, $51.3 \%$ reported using oral contraceptive for less than 12 months. The participants gathered information regarding oral contraceptive pills from drug leaflet $(82.5 \%)$, physicians $(62.4 \%)$, and pharmacists $(15 \%)$. Of the women, $66.2 \%$ reported taking pill at the same time every evening, and $40 \%$ of them started pill on the first day of menstrual period. $36.2 \%$ of them declared that they forgot to use oral contraceptive pills at least once in the last month. Although most of them $(82.5 \%)$ knew that they must take an extra pill if they forgot taken one pill in less than $12 \mathrm{~h}$, only $18.8 \%$ of them reported that they took an extra pill and used extra protection if they missed taking a pill for more than $12 \mathrm{~h}$. Of the women, $68.8 \%$ did not know what to do when they vomited within $2 \mathrm{~h}$ of taking pill. Only $\% 10$ of them took an extra pill when they had diarrhoea for more than $12 \mathrm{~h}$. Of the women, $31.3 \%$ had knowledge about contraindications of oral contraceptives pills. $43.8 \%$ of them did not know effects of smoking on utilization of oral contraceptive pills. The most commonly reported side effects by participants were weight gain $(35 \%)$, nausea $(28.8 \%)$, and increased appetite $(22.5 \%)$.

Conclusions: In this study, the women utilized oral contraceptive pills had less knowledge about some important issues that influence effectiveness of contraceptive pills. Pharmacists may take more professional response in education and monitoring of women utilized oral contraceptives pills.

Disclosure of Interest: None Declared.

\section{DI10}

Eosinophilia and pulmonary toxicity associated with lenalidomide

D. P. Parente ${ }^{1, *}$, M. Suarez Santamaría ${ }^{2}$,

J. M. Castro Dominguez ${ }^{2}$, E. Campelo Sanchez ${ }^{2}$,

M. P. Ascunce Saldaña ${ }^{2}$, N. Martinez Lopez de Castro², G. Piñeiro Corrales ${ }^{2}$

${ }^{1}$ Pharmacy, Hospital University Meixoeiro, ${ }^{2}$ Pharmacy, Hospital Meixoeiro, Vigo, Spain

Background and Objective: Pulmonary eosinophilia is a heterogeneous group of diseases characterized by the presence of pulmonary infiltrates and increased numbers of eosinophils in the lung or peripheral blood. There are drugs such as penicillins, cephalosporins, ACE inhibitors, neuroleptics, gold salts or NSAIDs, which can lead to lung eosinophils processes by a mechanism of type III and IV hypersensitivity.

This abstract presents a case of lenalidomide induced pulmonary eosinophilia. No many cases of lenalidomide induced pulmonary eosinophilia in the literature have been reported.

Setting and Method: We report a case of a 52 year-old female diagnosed with essential thrombocythemia jak2 positive in 2004 with subsequent transformation to myelodysplastic syndrome $5 \mathrm{q}$ deletion in 2010. She has been treated with hydroxyurea and thioguanine whitout response. In March 2011, the treatment was discontinued and she started with lenalidomide.

Results: Two weeks later the prescription of lenalidomide, the patient was admitted to our ER (emergency room). She presented febrile syndrome with pleuritic pain and pleural effusion associated, initially it wasinterpreted as pneumonia. Lenalidomide was discontinued, and she started empirical antibiotic treatment with symptomatic and clinical improvement.

After completion of antibiotic treatment and with culture-negative, lenalidomide was restarted. One week after, the patient presents clinical worsening and returns to our ER service with febrile syndrome and pleuritic pain. Antibiotic therapy was reintroduced and added empirical antifungal. Lenalidomide was discontinued again. Clinical and radiological improvement was achieved.

Currently the patient is treated with thioguanine without respiratory symptoms or progressive eosinophilia.

Conclusions: The correlation between eosinophilia, pulmonary radiological tests, respiratory symptoms, negative microbiological cultures and treatment with lenalidomide, they point to the likelihood of pulmonary toxicity secondary to the drug.

Disclosure of Interest: None Declared.

\section{DI11}

Fingolimod: one year observational evaluation use in neurology department of Henri Mondor hospital

\section{E. Rouimi ${ }^{1, *}$, P. Cesaro ${ }^{2}$, B. Berdougo ${ }^{1}$, A. Astier ${ }^{1}$, M. Paul ${ }^{1}$}

${ }^{1}$ Pharmacy, ${ }^{2}$ Neurology, Henri Mondor hospital, Creteil, France

Background and Objective: Following agreements contracted between Regional Health Agency and hospitals, the Drug and Sterile Medical Devices Committee (COMEDIMS) estimates medical requests of referencing new medicines and handles the revaluation of therapeutic strategies within the hospital.

To evaluate one year after Fingolimod admission, safety and efficiency of this new per os treatment of the multiple sclerosis (MS).

Setting and Method: A retrospective study concerning all patients treated by fingolimod and was conducted by a pharmacy resident in collaboration with neurologists. Data were collected from software Phedra, patient files and by interrogation of patients. They concerned safety (side effects, hepatic and ophtalmic function tests) and efficiency (number of MS exacerbations since institution of treatment, MRIs).

Results: Since December 2011, 10 patients received fingolimod according to market approval indications and underwent a $24 \mathrm{~h}$ hospitalization, as expected in the label used (LU).

In safety terms, one bradycardia required the end of treatment for $1 / 10$ patients with pharmacovigilance report.

The results of the monitoring totalized 7 patients (no data for 2 patients where treatment introduction was too recent). The hepatic function tests were all normal. 1/7 patient showed visual disorders. A patient showed headaches, joint pains and pneumonia at J3. Two patients also described a loss of weight.

Despite the weak size of the sample, these results are to be put in parallel with the frequencies of side effects known from LU: headaches and vision disorders are very frequent $10 \%$. However for bradycardia, pneumonia and the loss of weight (expected frequency $1 \%$ ), was observed at an abnormally high frequency. The pharmacovigilance imputed bradycardia to an interaction with a secondary treatment (carbamazepin). 
In efficiency terms, no patient had clinical MS exacerbations under fingolimod since introduction. Unfortunately, after one year of treatment, a radiological evolution of the disease engendered interruption of the treatment for one patient. These results are encouraging in comparison with the patient percentage free of MS exacerbations in 12 months of the studies ( $83 \%$ ), nevertheless the hindsight is weak (5 in 13 months).

Conclusions: This evaluation consolidates the interest of the fingolimod in terms of clinical efficiency. The unwanted effects those are expected from the LU. COMEDIMS decided to keep fingolimod in hospital. This evaluation also allowed to highlight the difficulty collecting data of monitoring when this one is made in town.

Disclosure of Interest: None Declared.

\section{DI12}

Patterns of use of panitumumab in a single academic institution

A. Pilon ${ }^{1}$, A. El Aatmani ${ }^{1}$, P. Barthelemy ${ }^{2}$, I. Asmane ${ }^{2}$, V. Juif ${ }^{3}$, B. Gourieux ${ }^{1}$, D. Levêque ${ }^{1, *}$

${ }^{1}$ Pharmacy, ${ }^{2}$ Oncology Department, ${ }^{3}$ Hepatogastroenterology Department, Hôpitaux Universitaires de Strasbourg, Strasbourg, France

Background and Objective: Panitumumab is a monoclonal antibody approved for treatment of wild-type KRAS, metastatic colorectal cancer. Data relative to its use in clinical practice are scarce. The goal of our study was to examine the pattern of panitumumab use in a single academic centre

Setting and Method: We identified all consecutive metastatic colorectal cancer patients given panitumumab between December 2007 and February 2013 in our institution through the pharmacy database. All medical files were retrospectively investigated to describe the patterns of panitumumab prescription and administration.

Main outcome measures: Recorded data included patients' demographics and medical history, patterns of panitumumab prescription. Safety data were analyzed from patients' charts as well as outcome. Results: We identified 25 patients with a median age of 64 years (range 47-80). The KRAS status was wild-type (WT) in $21 / 21$ patients (N/A in 4). The median number of metastatic organs was 2 (range 1-4). Most (21/25) of the patients had received prior chemotherapy for metastatic disease with either 5-fluorouracil, leucovorin and oxaliplatin (FOLFOX) or with 5-fluorouracil, leucovorin and irinotecan (FOLFIRI). Seventeen $(68 \%)$ patients had been pretreated by cetuximab and among them, 4 had discontinuated the drug for hypersensitivity reactions. Panitumumab $(6 \mathrm{mg} / \mathrm{kg})$ was given every 2 weeks as first-line therapy in $4(16 \%)$ patients with FOLFOX or as 5th line chemotherapy on average (range 2-9 line) in 21 (84\%) patients. Panitumumb was used in last line for 10 (40 \%) patients. For the 21 pretreated patients, panitumumab was given as monotherapy in $11(44 \%)$, or combined with either FOLFIRI or irinotecan, capecitabine, 5-fluorouracil in $3(12 \%)$ and $7(28 \%)$, respectively. The median treatment duration for patients who had discontinuated treatment (21/24) was 2.5 months (range, 0.5-8 months), with a median of 5 cycles (range 1-11). Discontinuations occurred due to response $(n=3)$, disease progression $(n=17)$ and adverse effects $(\mathrm{n}=1)$. Panitumumab-related toxicities included skin reactions $(\mathrm{n}=18 / 25)$ resulting in one drug discontinuation. Six $(24 \%)$ patients had preventive treatment with doxycycline. One patient $(4 \%)$ achieved a complete response and 2 patients $(8 \%)$ demonstrated a partial response.

Conclusions: Panitumumab was mostly used in highly pretreated patients with metastatic colorectal cancer among them half had progressed on cetuximab. Overall, panitumumab displayed minimal activity. Disclosure of Interest: None Declared.

\section{DI13}

Computerized pharmaceutical interventions: analysis and perspectives

F. Bringer ${ }^{1}$, S. Pelegrin ${ }^{1}$, M. Ponrouch ${ }^{1}$, D. Rosant ${ }^{1}$, I. Roch-Torreilles ${ }^{1}$, P. Rambourg, ${ }^{1, *}$

${ }^{1}$ Saint-Eloi/Gui de Chauliac Pharmacy unit, Dispensing and Clinical Pharmacy Department, University Hospital, Montpellier, France

Background and Objective: The computerization of the medication circuit in its different stages: prescription, dispensation and administration, is a privileged way for improving the patients care. The pharmaceutical validation is facilitated and allows the pharmacist to identify and typify his interventions.

This study aims to analyse the pharmaceutical validation through the pharmaceutical interventions (IP) in the University Hospital of Montpellier.

Setting and Method: Using a query tool, extraction of the number and type of IP has been done with the prescriptions software "DxCare". Refusal comments were analysed over a period of 6 months (January-June 2013).

Main outcome measures: These comments were classified as: dosage adjustment, addition (new prescription), recommended stop, contraindications, double, side effects, error of prescription unit, error of administration frequency, interaction, error of indication, not complying with the standards, under-dosing, therapeutic monitoring, overdose, stopped treatment, inappropriate route of administration.

Results: 2,441 computerized prescriptions were analysed. The main reasons for refusal are: prescription unit errors $(46.3 \%)$, double $(15.5 \%)$, stopped treatment $(7.5 \%)$, error of administration frequency $(6.3 \%)$, dosage adjustment $(5.8 \%)$, inappropriate route of administration $(4.3 \%)$ and overdose $(3.8 \%)$.

Errors of prescription units and the omissions of stopped treatment can be attributed to a training problem of prescribers to DxCare software. Poor agreement between prescribers from different disciplines can explain doubles when transferring patients from one ward to another. IP on errors of administration frequency mainly concerned long-acting durgs. For dosage adjustments, in some cases, doses were not adapted to the weight or renal clearance.

Confusion between the IM and IV route resulted in errors on the route of administration. And finally, the majority of overdoses involved prescriptions of paracetamol and tramadol. In fact, the maximum doses were often overtaken by the many pharmaceutical specialities containing these active ingredients.

Conclusions: IP, mainly the refusals, identify the prescriptions errors and the improvements to do in terms of training and pharmaceutical knowledge to provide to prescribers.

It would be interesting to continue this study by analysing the IP acceptance rate as well as the reduction of drug-related iatrogenia.

Disclosure of Interest: None Declared.

\section{Pharmacoepidemiology}

\section{PE03}

Incidence of and risk factors for severe hypoglycemia in treated type 2 diabetes mellitus patients in the United Kingdom

S. G. Bruderer,2,*, S. S. Jick ${ }^{3}$, G. Bader ${ }^{4}$, R. Schlienger
C.
C. Meier

${ }^{1}$ Basel Pharmacoepidemiology Unit, Division of Clinical Pharmacy and Epidemiology, Department of Pharmaceutical Sciences, University of Basel, ${ }^{2}$ Hospital Pharmacy, University Hospital Basel, 
Basel, Switzerland, ${ }^{3}$ Boston Collaborative Drug Surveillance Program, Boston University School of Public Health, Lexington, United States, ${ }^{4}$ Global Medical Affairs, Diabetes, ${ }^{5}$ Global Clinical Epidemiology, Novartis Pharma AG, Basel, Switzerland

Background and Objective: Several risk factors are known for severe hypoglycemia $(\mathrm{SH})$ in patients with type 2 diabetes mellitus (T2DM), but data on incidence rates (IRs) of and risk factors for $\mathrm{SH}$ from a 'real world' setting are scarce. We therefore aimed at assessing the proportion of patients with, and IRs of recorded SH, and to further characterize potential risk factors for $\mathrm{SH}$.

Setting and Method: We performed a retrospective cohort study in T2DM patients derived from the UK-based General Practice Research Database and assessed IRs (with $95 \%$ confidence intervals [95\% CIs]) of incident SH per 10,000 person-years (PYs). In a nested casecontrol analysis we compared cases (patients with recorded $\mathrm{SH}$ ) with matched (1:10) controls without hypoglycemia during follow-up. Using conditional logistic regression models we computed adjusted odds ratios (ORs) with $95 \%$ CIs of $\mathrm{SH}$ in association with various potential risk factors of interest.

Results: Of 130,761 patients with T2DM (mean age $61.7 \pm$ 13.0 years), $690(0.5 \%)$ were identified with an incident $\mathrm{SH}$ event during follow-up. The estimated IR overall was 12.0 (95\% CI 11.1-12.9) per 10,000 PYs. IRs were markedly higher in insulin users (49.6 [44.1-55.9] per 10,000 PYs) than in patients not using insulin $(8.0$ [7.3-8.8] per 10,000 PYs). Sex and cardiovascular disease did not alter the SH risk. Risk factors associated with an increased SH risk included age (adjusted [adj.] OR 2.3 [95 \% CI 1.7-3.1] in patients $\geq 75$ years vs. those aged 20-59 years), cognitive impairment/dementia (adj. OR 2.0 [1.4-2.9]), renal impairment (adj. OR 1.3 (1.0-1.7), current sulfonylurea (SU) use (adj. OR 4.5 [3.5-5.6]), and current insulin use (adj. OR 11.8 [9.0-15.5); current metformin use was associated with a slightly decreased SH risk (adj. OR 0.8 [0.6-1.0]).

Conclusions: In this large UK cohort of T2DM patients, age, cognitive impairment/dementia, renal impairment and current use of insulin or SU significantly increased, while current metformin use decreased the risk of developing SH. Overall, these findings are in concordance with results from previous studies.

Disclosure of Interest: None Declared.

\section{PE04}

Use of benzodiazepines and history of falls in older people admitted to acute care settings in Australia

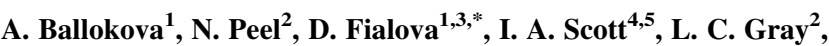
R. E. Hubbard ${ }^{2}$

${ }^{1}$ Department of Geriatrics and Gerontology, 1st Faculty of Medicine, Charles University in Prague, Prague, Czech Republic, ${ }^{2}$ Centre for Research in Geriatric Medicine, School of Medicine, University of Queensland, Brisbane, Australia, ${ }^{3}$ Department of Social and Clinical Pharmacy, Faculty of Pharmacy in Hradec Kralove, Charles University in Prague, Hradec Kralove, Czech Republic, ${ }^{4}$ School of Medicine, The University of Queensland, ${ }^{5}$ Department of Internal Medicine and Clinical Epidemiology, Princess Alexandra Hospital, Brisbane, Australia

Background and Objective: Changes in pharmacokinetics and pharmacodynamics of benzodiazepines (BZDs) during ageing may increase the pharmacological potential of these medications to cause adverse outcomes. Yet BZDs are still widely prescribed for older people. This study investigates BZDs in acute care settings and explores the associations between use of these medications and falls. Setting and Method: This is a prospective cohort study of 1,412 patients aged over 70 years admitted to general medical, orthopaedic and surgical wards in 11 acute care hospitals in 2 states of Australia. Patients were recruited between July 2005 and May 2010. The interRAI Acute Care assessment tool was used to collect data including medications. Analyses were conducted using Pearson's Chi square test and logistic regression models.

Main outcome measures: Associations between the use of BZDs and history of falls (at least one fall in the previous 90 days) were determined.

Results: Out of 1,412 patients $(61.6 \%$ women, mean age $81.0 \pm$ 6.8), $146(10.3 \%)$ were on BZDs at admission assessment. The three most frequently used BZDs were oxazepam (33.6 \% of BZD users), temazepam $(32.3 \%)$, and diazepam $(16.8 \%)$. There was no statistically significant association between the use of BZDs and history of falls, comparing users and non-users. Patients on diazepam were significantly more likely to have fallen than all other BZDs users (70.0 \% and $36.1 \%$, respectively, $p=0.002)$ and in particular when compared to oxazepam users $(70.8 \%$ and $25.0 \%$, respectively, $p=0.001)$. Users of oxazepam were less likely to fall in the previous 90 days compared to all other BZDs users $(25 \%$ and $49 \%$, respectively, $p=0.007)$. Multivariate logistic regression model adjusting for cognition, functional status, age and gender confirmed that use of diazepam was positively associated with falls comparing to all other BZDs users (OR 3.7; CI 1.4-9.9, $p=0.008$ ).

Conclusions: The findings of our study suggest that use of BZDs is still common $(10 \%)$ in older patients admitted to acute care settings in Australia. There were differences in negative outcomes of particular BZDs in relation to falls. Use of diazepam was independently associated with falls. The indications for, and selection of, a particular BZD to older patients should be carefully evaluated.

Disclosure of Interest: None Declared.

\section{PE05}

Use of benzodiazepines and Z-durgs in older nursing home residents in Europe and their association with adverse outcomes: results from the EU SHELTER project

A. Ballokova ${ }^{1}$, D. Fialova ${ }^{1,2, *}$, N. Peel ${ }^{3}$, L. C. Gray ${ }^{3}$, R. E. Hubbard ${ }^{3}$

${ }^{1}$ Department of Geriatrics and Gerontology, 1st Faculty of Medicine, Charles University in Prague, Prague, ${ }^{2}$ Department of Social and Clinical Pharmacy, Faculty of Pharmacy in Hradec Kralove, Charles University in Prague, Hradec Kralove, Czech Republic, ${ }^{3}$ Centre for Research in Geriatric Medicine, School of Medicine, The University of Queensland, Brisbane, Australia

Background and Objective: Use of hypnosedative drugs is common in older patients because of frequent anxiety and sleep problems. Sedative potential of benzodiazepines (BZDs) and Z-drugs (Z-Ds) can be affected by age-related pharmacological changes and lead to adverse drug outcomes, e.g. falls, cognitive impairment, day sleepiness. Our study focuses on differences in use of BZDs and Z-Ds in European countries and association with falls.

Setting and Method: A cross-sectional study conducted in 2009-2011 in 7 European countries and Israel included 4,156 nursing home $(\mathrm{NH})$ patients aged 65 years and older assessed at baseline for the EU SHELTER project. Data were collected using the interRAI Long-Term Care Facilities assessment tool and analysed using Pearson's Chi square statistics and multiple logistic regression models.

Main outcome measures: Associations between the use of different BZDs, Z-Ds and falls (at least one fall in the previous 90 days) were determined.

Results: Of 4,156 NH residents ( $73 \%$ women, mean age $83.4 \pm 9.4$ ), $1,113(26.8 \%)$ patients were prescribed BZDs and/or Z-Ds. The 
highest prevalence of BZDs and Z-Ds users was in Israel (44.1\%), France $(44.0 \%)$ and The Netherlands $(26.5 \%)$. The most frequently prescribed drugs in the BZDs and Z-Ds group were zopiclone $(17.8 \%)$, lorazepam $(17.7 \%)$ and oxazepam $(16.9 \%)$. There were significant differences in distribution of BZDs and Z-Ds across countries: brotizolam most frequently prescribed in Israel $(99.4 \%$ of brotizolam users), zolpidem in France (50.0 \%), alprazolam in France (46.7\%). 111 residents (10\% of BZD users, $2.6 \%$ of entire sample) used more than one BZD. There was no statistically significant association between the single BZD use and falls. When compared to nonuse, the use of multiple BZDs was positively associated with at least one fall in last 90 days (OR 1.6; CI 1.00-2.43, $p=0.047$ ); however no association with falls was found comparing to single BZD users.

Conclusions: Our findings demonstrate that the prevalence of use of BZDs and Z-Ds as well as prescribing patterns differ among the European countries. Taking multiple BZDs is independently associated with falls in older patients. Thus the prescription of BZDs and Z-Ds in older patients should be carefully evaluated.

Disclosure of Interest: None Declared.

\section{PE06}

Prevalence of medication-related hospitalizations: a systematic review and meta-analysis

\section{T. T. Souza ${ }^{1}$, R. R. Godoy ${ }^{1}$, I. Rotta ${ }^{1}$, P. K. Ziegelmann ${ }^{2}$,} F. Fernandez-Llimos ${ }^{3}$, C. J. Correr ${ }^{4, *}$

${ }^{1}$ Pharmaceutical Sciences Post-Graduate Program, Federal University of Parana, Curitiba, ${ }^{2}$ Biostatistics Department, Federal University of Rio Grande do Sul, Porto Alegre, Brazil, ${ }^{3}$ Social Pharmacy Department, University of Lisbon, Lisbon, Portugal, ${ }^{4}$ Pharmacy Department, Federal University of Parana, Curitiba, Brazil

Background and Objective: This study aimed to evaluate the prevalence of hospitalizations due to different types of harms caused by medications, in different populations, under different methods of data collection.

Setting and Method: Systematic review and meta-analysis of observational studies published up to August 2012 in databases Medline, EMBASE, International Pharmaceutical Abstracts (IPA), Science Direct, Scopus, Lilacs, SciELO and Web of Science.

Main outcome measures: Prevalence of hospitalizations due to adverse drug events (ADE), drug-related problems (DRP), adverse drug reactions (ADR), accidental drug intoxication (ADI), medication errors (ME), drug interactions (DI), inappropriate medications use (IM), abrupt reduction of dose (ARD), unnecessary medication use (UM), omission of treatment (OT), non-adherence to treatment (NA) and therapeutic failure (TF).

Results: It was included 49 studies evaluating medication-related hospitalizations, totaling a population of $91,494,600$. The prevalence of hospitalizations due ADE was $1.5 \%$ (95 \% CI 0.5-3.8\%) and the elderly were the most affected population $(7.6 \%, 95 \% \mathrm{CI}$ 0.2-74.2\%). Regarding DRP, $6.3 \%$ (95\% CI 2.1-17.8\%) of patients were hospitalized due to DRP and the population with highest prevalence were the elderly $(21.4 \%$, $95 \%$ CI $8.8-43.4 \%)$. The prevalence of hospitalization due ADR was $6.3 \%(95 \% \mathrm{CI}$ $3.8-10.3 \%)$ and the elderly were the population with highest prevalence $(13.1 \%$, $95 \%$ CI $6.5-24.9 \%)$. Furthermore, $1.6 \%$ (95\% CI $0.9-2.6 \%$ ) of patients were hospitalized due to ADI, $8.7 \%$ (95\% CI 5.8-12.9\%) due to $\mathrm{ME}$ and $0.8 \%(95 \% \mathrm{CI}$ $0.5-1.3 \%$ ) due to DI. The prevalence of hospitalization due to use of inappropriate medications and unnecessary medications were $0.9 \%$ (95\% CI $0.5-1.9 \%)$ and $0.2 \%$ (95\% CI $0.0-1.2 \%)$, respectively. Additionally, $1.4 \%$ (95\% CI $0.8-2.3 \%$ ) of patients were hospitalized due to ARD, $3.2 \%$ (95\% CI 1.6-6.3\%) due to
OT and $2.5 \%$ (95 \% CI 1.7-3.8\%) due to NA. Concerning to TF, the prevalence of hospitalization due to TF was $3.7 \%(95 \% \mathrm{CI}$ 2.3-6.0\%). The method that detected the highest prevalence of medication-related hospitalizations was chart review and patient interview.

Conclusions: The results demonstrate that the drug-related morbidity and mortality remain a significant problem of public health with great impact on society and on health systems, responsible for a high proportion of hospitalizations. It is evident the need of preventive actions and early intervention aiming to reduce the harm caused by medications.

Disclosure of Interest: None Declared.

\section{PE07}

Trends of allopurinol use in Hungary between 2003 and 2012; Is the increase reasonable?

M. Matuz ${ }^{1,2}$, R. Benkö ${ }^{1,2}$, R. Viola ${ }^{1,2, *}$, I. Csóka ${ }^{1,3}$, G. Soós ${ }^{1,2}$

${ }^{1}$ Hungarian National Committee of Pharmaceutical Care, Budapest, ${ }^{2}$ University of Szeged, Department of Clinical Pharmacy, ${ }^{3}$ Hungarian National Committee of Pharmaceutical Care, Szeged, Hungary

Background and Objective: Hyperuricaemia has been recognised as an independent cardiovascular risk factor. This finding undermine the previous theory of treating only symptom positive patients (i.e. those with gouty arthritis). Allopurinol was marketed in 1972 in Hungary and since then available under one brandname, in two strengths $(100 \mathrm{mg}$ and $300 \mathrm{mg}$ ). Recently, the prevalence of hyperuricaemia was found $12 \%$ in women and $16 \%$ in men among Hungarian inhabitants.

Assess the last ten years' allopurinol consumption in Hungary and estimate the treatment rate of hyperuricaemic patients.

Setting and Method: The study period was between 2003 and 2012. Data on the number of dispensed allopurinol packages was obtained from the National Health Fund Administration (NHFA). Crude data was converted into defined-daily doses (DDD) according to the newest version of the ATC/DDD index.

Main outcome measures: allopurinol use in DDD per 1,000 inhabitants and per year (2003-2012), number of people who redeemed allopurinol in 2012.

Results: In 2003, allopurinol use was 3.9 DDD per 1,000 inhabitant and per year, expressed a gradual, linear increase and reached 12.5 DDD per 1,000 inhabitant and per year in 2012. The product with lower allopurinol content $(100 \mathrm{mg})$ shared $58 \%$ in the first year of analysis, while in the last year its proportional use dropped to $32 \%$. In $20123.9 \%$ of the population received minimum one allopurinol package.

Conclusions: The significant increase of the allopurinol consumption shows clearly the recognised importance of the problem, but on the other hand indicates ongoing undertreatment of hyperuricaemic patients.

Disclosure of Interest: None Declared.

\section{PE08}

Prevalence and risk factors for adverse drug events: a systematic review and meta-analysis

T. T. Souza ${ }^{1}$, R. R. Godoy ${ }^{1}$, P. K. Ziegelmann ${ }^{2}$, F. Fernandez-Llimos ${ }^{3}$, C. J. Correr ${ }^{4, *}$

${ }^{1}$ Pharmaceutical Sciences Post-Graduate Program, Federal University of Parana, Curitiba, ${ }^{2}$ Biostatistics Department, Federal University of Rio Grande do Sul, Porto Alegre, Brazil, ${ }^{3}$ Social Pharmacy Department, University of Lisbon, Lisbon, Portugal, ${ }^{4}$ Pharmacy

Department, Federal University of Parana, Curitiba, Brazil 
Background and Objective: This study aimed to evaluate the prevalence, characteristics and consequences of adverse drug events (ADE) to different populations and health care settings, under different methods of data collection and distinct conceptual definitions, besides to evaluate the pharmacological groups involved and the population risk factors that favor its appearance.

Setting and Method: Systematic review and meta-analysis of observational studies published up to August 2012 in databases Medline, EMBASE, International Pharmaceutical Abstracts (IPA), Science Direct, Scopus, Lilacs, SciELO and Web of Science.

Main outcome measures: Prevalence, severity and avoidability of $\mathrm{ADE}$, prevalence of hospitalizations and death due to ADE, pharmacological groups involved in occurrence of $\mathrm{ADE}$ and the population risk factors that influence the emergence of ADE.

Results: It was included 206 studies evaluating ADE, totaling a population of $613,022,838$. The global prevalence of ADE was $7.6 \%$ (95\% CI 4.3-12.9\%). The country with highest prevalence of ADE was Spain $(16.7 \%, 95 \%$ CI 1.8-69.1\%) followed by Brazil (13.6 \%, $95 \%$ CI 2.2-53.0 \%) and the setting with the highest ADE prevalence was the primary care and community $(28.6 \%$, $95 \%$ CI $12.2-53.6 \%$ ). Regarding age group, elderly patients had the highest prevalence of $\operatorname{ADE}(17.6 \%, 95 \%$ CI 10.9-27.3\%). The method that detected the highest ADE prevalence was interview (18.9\%, $95 \%$ CI 16.8-21.1\%). The prevalence of hospitalization and death due to ADE was $1.5 \%(95 \%$ CI $0.5 \%>3.8 \%)$ and $0.2 \%(95 \% \mathrm{CI}$ $0.1-0.8 \%$ ), respectively. Were identified many heterogeneity sources in the prevalence meta-analysis of $\mathrm{ADE}$, being the main ones: high methodological diversity among the studies, given by the variety of detection methods used, health care local, subpopulations analyzed and definitions and terminological classifications adopted. The main pharmacological groups involved were central nervous system, antiinfectives and the antineoplastic and immunomodulators agents. The main risk factors that influence the emergence of ADE were age greater than 65 years, co morbidities, usage of four or more medications and presence of renal disease.

Conclusions: The high prevalence of ADE in many health care settings demands evaluation and stratification of population risk, preventive actions and early interventions. It must be taken strategies to ensure an efficient medication therapy management in health care settings, aiming to contribute with the decreasing of social and economic impact of harms caused by medications.

Disclosure of Interest: None Declared.

\section{PE09}

Hypersensitivity reactions and cardiac toxicity related to Trastuzumab in HER2 positive breast cancer patients

L. R. Ayres, ${ }^{1, *}$, M. S. D. A. Campos ${ }^{1}$, T. D. O. Gozzo ${ }^{2}$, A. Q. Ungari ${ }^{3}$, J. M. de Andrade ${ }^{4}$, L. R. L. Pereira ${ }^{1}$

${ }^{1}$ Pharmaceutical Sciences, School of Pharmaceutical Sciences of Ribeirão Preto, University of São Paulo, ${ }^{2}$ School of Nursing of Ribeirão Preto, University de São Paulo, ${ }^{3}$ General Hospital of the Faculty of Medicine of Ribeirão Preto, University of São Paulo, ${ }^{4}$ Faculty of Medicine of Ribeirão Preto, University of São Paulo, Ribeirão Preto, Brazil

Background and Objective: Trastuzumab use is associated with an increased survival rate in HER2 positive breast cancer patients. Trastuzumab is generally well tolerated, however, it is related to some toxicity, especially hypersensitivity reactions and cardiac toxicity (CT). Hypersensitivity reactions and CT may require treatment discontinuation depending on the severity, which can deprive patients of the benefits of this therapy. Therefore, this study aimed to identify the incidence and severity of hypersensitivity reactions and CT related to trastuzumab in clinical practice.

Setting and Method: This study was performed in the Mastology Sector of the General Hospital of Ribeirão Preto. We retrospectively reviewed the medical records of patients without distant metastasis that started trastuzumab between 2007 and 2011 in a general hospital. Main outcome measures: Cardiac toxicity (CT) was defined as symptomatic heart failure or a decrease in left ventricular ejection fraction (LVEF) by $\geq 10 \%$ compared to the first echocardiogram measurement or to $<50 \%$ at any time. Hypersensitivity reactions were classified according to the Common Terminology Criteria for Adverse Events v4.0 of the National Cancer Institute.

Results: We analyzed the records of 79 patients. CT developed in $26(32.9 \%)$ patients, being the LVEF decline by $\geq 10 \%$ observed in $21(26.6 \%)$, a decreased to $<50 \%$ in four $(5.1 \%)$ and one (1.2\%) was symptomatic without LVEF decline. Thirteen (16.4\%) patients discontinued permanently the treatment, three $(3.8 \%)$ discontinued temporarily and $10(12.6 \%)$ finished it without interruption. Considering the hypersensitivity reactions, they were classified in the Immune System Disorders group as allergic reactions. It occurred in $16(20.3 \%)$ patients, half of them developed grade I allergic reactions (no intervention required) and the other half developed grade II (when intervention or temporary interruption of the medication is needed). Nine out of 16 patients experienced infusion reactions.

Conclusions: Although most patients finished their treatment, CT led to trastuzumab discontinuation in a significant proportion of patients suggesting the need of a closer cardiac monitoring. Trastuzumab is related to mild-to-moderate hypersensitivity reactions which are managed by temporarily interrupting the infusion and administering supportive care for symptoms. Accurate grading of reactions is essential in determining how to proceed with treatment.

Disclosure of Interest: None Declared.

\section{PE10}

Spontaneous adverse drug reactions and off-label prescribing in children in the Slovak Republic

\section{J. Slažneva ${ }^{1, *}$, R. Kamenská ${ }^{2}$, M. Kuželová ${ }^{1}$}

${ }^{1}$ Pharmacology and Toxicology, Faculty of Pharmacy, Comenius University, ${ }^{2}$ Section of Drug Safety and Clinical Trials, State Institute for Drug Control, Bratislava, Slovakia

Background and Objective: Drugs prescribed and used in children outside the terms of their product license is known as off-label prescribing. As off-label prescribing is usually lacking any evidence on safety, major concerns rise when using in paediatric population. The aim of study was to investigate the extent and characteristics of offlabel prescribing in the context of adverse drug reactions (ADRs) in the Slovak Republic among children.

Setting and Method: A retrospective analysis of spontaneous ADRs reported to State Institute for Drug Control in the Slovak Republic during 2006-2012 concerning drugs prescribed for patients younger than 18 years was performed. Each ADR was classified with respect to seriousness and type of reaction. Off-label drug use was defined as prescription for children outside the recommended age group listed in the Summary of Product Characteristics. ADRs related to vaccines, intravenous replacement solutions, blood products, dietary supplements were excluded.

Main outcome measures: Proportion of reported ADRs associated with off-label prescribing in children.

Results: A total of 1904 ADR reports for children aged 0-17 years were submitted to Slovak national database over 7 years, and $89 \%$ of 
these reports were for vaccines. According to our inclusion criteria 217 patient-linked reports were identified and analysed corresponding to 409 ADRs of which $56 \%$ were serious and $3 \%$ fatal. The most common clinical manifestations of ADRs were exanthema (18\%), fever $(5 \%)$ and vomiting (3\%). Anti-infectives for systemic use (42\%), antineoplastic and immunomodulating agents (16\%) and drugs for nervous system (16\%) were most frequently suspected as a cause of reported ADR. The highest occurrence of serious paediatric ADRs were reported for antineoplastic and immunomodulating agents, drugs for respiratory system and alimentary tract and metabolism medications (range 75-82\%). $9 \%$ of ADR reports were associated with off-label use, more frequently with serious than nonserious ADRs and one fatal case. Etoposide was the most frequent drug used off-label with respect to age associated with ADRs.

Conclusions: This study has demonstrated that off-label prescribing for paediatric patients is common among drugs reported to have caused an ADR.

Study was supported by grant FaF UK/8/2013.

Disclosure of Interest: None Declared.

\section{PE11}

Use of drugs for peptic ulcer and gastro-oesophageal reflux disease (GORD) in older patients

\author{
K. H. Halvorsen ${ }^{1, *}$, L. Småbekke ${ }^{1}$, A. G. Granås ${ }^{2}$ \\ ${ }^{1}$ Pharmacy, University of Troms $\varnothing$, Troms $\varnothing,{ }^{2}$ Pharmacy, Faculty of \\ Health Science, Oslo, Norway
}

Background and Objective: According to the Norwegian prescription database drugs for GORD are prescribed to $16.3 \%$ of the general older population. Similar figures from Sweden estimate about $19.5 \%$ use. In this study we aimed to explore the pattern of use of drugs for GORD in older patients in home care service and nursing homes.

Setting and Method: Cross-sectional study based on 2009 data including patients $\geq 65$ years from home care services and nursing homes using multi-dose dispensed drugs.

Main outcome measures: Prevalence, type of drugs, and impact of care setting on exposure to drugs for GORD were calculated, adjusting for patients age, gender and number of drugs used.

Results: The study population included 11,254 patients, 2986 patients in nursing homes (mean age 85.3 years, $72 \%$ women) and 8 268 patients in home nursing services (mean age 83.0 years, $69 \%$ women). On average patients used 5.7 multi-dose dispensed drugs. In total, $21.3 \%$ of patients used drugs for GORD. Most commonly prescribed drugs were esomeprazole $(41.5 \%)$, pantoprazole $(25.1 \%)$ and omeprazole $(13.7 \%)$. No differences in total drugs use between settings were observed.

Conclusions: The use of drugs for GORD in older patients receiving multi-dose dispensed drugs in home care service and nursing homes is higher compare to the general older population. These differences needs to be further explored.

Disclosure of Interest: None Declared.

\section{PE13}

Electronic health record improves adverse drug reactions reports.

L. Canadell Vilarrasa ${ }^{1, *}$, M. Mota Almeida ${ }^{1}$,

R. S. Almeida Perez ${ }^{1}$, J. Torrent Pou ${ }^{1}$, L. Castillo Palomares ${ }^{1}$,

M. Martin Marques ${ }^{1}$, M. Olona ${ }^{1}$, G. Garcia Pardo ${ }^{1}$,

M. Canela Subirada ${ }^{1}$

${ }^{1}$ Farmacia, Hospital Universitario Joan XXIII, Tarragona, Spain
Background and Objective: Pharmacovigilance systems are needed to improve drug safety and healthcare professionals play a vital role by reporting adverse drug reactions (ADR). Individual case reports can help in detecting new safety signals and identify risk factors for ADRs.

The introduction of an electronic health record in hospitals can modify the adverse drug reactions reporting. So we decided, to make a quantitative and qualitative analysis of the adverse drug reactions (ADRs) reported after and before the introduction of an electronic health record in the hospital in 2010. We also analyzed the incidence of ADRs in hospital discharge reports, as well as drugs and clinical services involved in these two periods.

Setting and Method: We conducted a retrospective study about the notification of ADR in our hospital before and after implementation of an HER in 2010. We collected data of two periods (2008-2009 and 2011-2012).

Main outcome measures: ADRs were detected in patients with discharge reports including event codes as defined by the IDC-9-CM system, using the minimum basic data set (MBDS).Global percentage of ADR per patient discharge, ADR percentage per hospital service, hospital departments with more notification and ADRs types were analysed.

Results: The percentage of ADR per discharge report in 2008/09 and 2011/12 were respectively $1.07 \%$, and $2.25 \%$. The hospital departments with highest percentage of ADR per discharge were haematology $(18.1 \%)$ and emergency $(10.2 \%)$ in $08 / 09$ and haematology $(20.8 \%)$ and pneumology $(14.8 \%)$ in $11 / 12$.

The ADR notification in medical departments increased a $10.63 \%$ (from $70.3 \%$ to $77.8 \%$ ) but in surgical departments decreased a $24.92 \%$ (from $29.7 \%$ to $22.3 \%$ ).

Haematology (22\% in 08/09 and $15.5 \%$ in 09/10) and Internal Medicine (15.5\% in $08 / 09$ and $24 \%$ in $11 / 12)$ were the hospital services with more notifications in both periods.

Drugs most commonly involved in ADR were: systemic corticoids $(26.6 \%)$, diuretics, $(13.8 \%)$ heart rhythm drugs $(7.6 \%)$ and anticoagulants $(6.2 \%)$. No significant differences with drugs were detected in both periods. A high proportion of ADEs were due to a small number of drugs (14) and were preventable in a high percentage $(85 \%)$.

Conclusions: HER implementation have been a good tool to improve the ADR notification and have managed to double our rates of recording. Medical departments are the most involved in the notification process and we must encourage surgical departments. Effective safety practices directed to reduce the incidence of ADRs can be introduced.

Disclosure of Interest: None Declared.

\section{PE14}

COPD: severity risk factors (RF) and evaluation of medication use

N. M. Vandevelde ${ }^{1, *}$, P. M. Tulkens ${ }^{1}$, I. Philippart ${ }^{2}$, N. Coppens ${ }^{3}$, H. Rodriguez-Villalobos ${ }^{4}$, J. Cadrobbi ${ }^{3}$, G. Liistro ${ }^{4}$, J.-P. d'Odemont ${ }^{2}$, A. Boel ${ }^{5}$, P. Jordens ${ }^{5}$, Y. Valcke ${ }^{6}$, H. Francart ${ }^{6}$, F. Van Bambeke ${ }^{1}$

${ }^{1}$ Louvain Drug Research Institute, Université catholique de Louvain, Brussels, ${ }^{2}$ CHR Mons-Warquignies, Mons, ${ }^{3}$ clinique Ste Elisabeth, Namur, ${ }^{4}$ Cliniques universitaires St Luc, Université catholique de Louvain, Brussels, ${ }^{5}$ OLV Ziekenhuis, Aalst, ${ }^{6} \mathrm{AZ}$ Nikolaas, St Niklaas, Belgium

Background and Objective: To evaluate COPD RF for severity (respiratory symptoms, hospitalization length) and drugs at admission in patients hospitalized with Acute Exacerbation of Chronic Bronchitis (AECB).

Setting and Method: Post-hoc analysis of 2 years retrospective case series study of patients hospitalized for exacerbation of COPD in 5 
Belgian hospitals (main criterion for enrolment: S. pneumoniae isolated in the sputum; microbiological data not included in the present analysis). Multivariate analysis using Fisher's exact test + Woolf approximation to calculate OR with $95 \% \mathrm{CI}$ and $p$-value; Analysis of severity (GOLD; hospitalization length), RF (age, tobacco addiction, gender, living place); medications for COPD or other pathologies.

Main outcome measures: significant RF of severity; medications used for treatment.

Results: Among 43 COPD patients (age 47-97; 29/14 M/F), $49 \%$ were GOLD3-4, $33 \%$ hospitalized $>10$ days, $56 \%>65$ years old, and $75 \%$ smoked $>20$ Pack Years [PY]. RF for severity were age $(p<0.01)$, male sex $(p=0.054)$, living out of nursing homes $[\mathrm{NH}]$ $(p=0.06)$. GOLD3-4 was associated with prolonged hospitalization $(p=0.052)$, which was more frequent for smokers $>20 \mathrm{PY}(p<0.05)$ or patients coming from NH $(p<0.01)$. Smokers $>20 \mathrm{PY}$ were mainly found in psychiatric institutions $[\mathrm{PI}](p<0.05)$ and light smokers in $\mathrm{NH}(p<0.001)$ or old patients $(p<0.05)$. Intake of bronchodilators (short-acting, long-acting or combined) or of inhaled corticosteroids at admission was associated with higher severity ( $p<0.01$ ); long-acting bronchodilators or inhaled corticosteroids, with shorter hospitalization $(p<0.05)$. Among other medications, proton pump inhibitors were more frequently taken by GOLD3-4 patients $(p=0.055)$ and inhaled corticoids in elderly $(p<0.01)$ or in patients smoking $\leq 20 \mathrm{PY}(p<0.001)$, and long-acting bronchodilators in elderly $(p<0.01)$.

Conclusions: Age and male sex are RF for COPD severity and smoking increases hospitalization length. NH patients were less severe, possibly because of lower tobacco addiction and/or better compliance to long-term treatment. High use of short-acting bronchodilators in severe patients suggests non-adherence to GOLD guidelines and/or poor compliance to long-term treatment. Smoking cessation in PI should be encouraged to prevent severe COPD development.

Disclosure of Interest: None Declared.

\section{Pharmacoeconomics}

\section{PEC05}

Pharmaco-economic impact of the implementation of dispensing assistance software for anti-infective drugs

\section{A. Glanard ${ }^{1, *}$, F. Roullet-Renoleau ${ }^{1}$, L. Drapeau ${ }^{1}$, B. Politis ${ }^{1}$, D. Gueylard Chenevier ${ }^{1}$}

${ }^{1}$ Pharmacy, Centre Hospitalier Intercommunal du Pays de Cognac, Cognac, France

Background and Objective: To improve quality of dispensing antiinfective drugs (AID), the local Anti-Infective Committee decided to control AID deliveries. This is a time-consuming task, requiring a double prescription. The aim of this before-after study was to determine if a Dispensing Assistance Software (DAS) is more effective than usual circuit.

Setting and Method: The study was conducted within the Pneumonology department. Each period of study lasted 11 days. Before DAS, a double system of prescription/dispensation (nominative paper and computerized prescription(PHARMA ${ }^{\circledR}$ software) was used for prescription and delivery of AID. After, prescription was only made using the prescription software.

Main outcome measures: Efficiency was evaluated from quality of prescriptions; these were checked on 3 categories of nonconformity (NC): 14 items linked to regulation (NC1); 13 items linked to respect of local Good Practice Referential (NC2); number of paper and computerised prescriptions mismatches (NC3).
Time required to validate AID prescriptions and dispense AID was measured every day. These measures were multiplied by the hourly-cost of each staff category to obtain the direct cost of this process.

The ICER (Incremental Cost-Effectiveness Ratio) was calculated to value the cost of each avoided NC.

Results: Patients groups before $(n=24)$ and after $(n=21)$ were not different. Consumptions of all antibiotic classes were similar. The number of global nonconformities $(\mathrm{NC}=\mathrm{NC} 1+\mathrm{NC} 2+\mathrm{NC} 3)$ was different before and after DAS (321NC vs $233 \mathrm{NC}$ corresponding respectively to 39 and 41 lines of prescriptions) - this result is significant even if not taking into account $34 \mathrm{NC} 3$ only present in the first period ( 287 vs $233 p<0.0001$ ). Main differences were explained by NC1 (before 83; after $41 p<0.0001$ ). Only $83.80 \%$ of AID paper prescriptions matched the AID computerised prescriptions (before $\mathrm{n}=39$ ). Overall time to validate and dispense AID was inferior in after period (396 min/57 deliveries vs $302 \mathrm{~min} / 69$ deliveries $p<0.001)$. Nevertheless DAS was more time consuming for pharmacists $(188 \min$ vs $212 \min p<0.001)$ and less time consuming for PA (208 min vs $90 \min p<0.001)$. Even if hourly-costs were higher for pharmacists, the global cost was inferior after DAS $167 €$ (132€ for a pharmacist and $35 €$ for an PA) versus $198 €$ (117€ for a pharmacist and $81 €$ for an PA) before.

The ICER was equal to $0.574 €$ per avoided NC.

Average time of prescription validation was significantly different depending on the use or not of DAS. We noticed that there were more changes of prescriptions in the 2 nd period $(p=0.05)$.

Conclusions: Each prescription is validated at least once a day that increase quality prescription analyses. It allows pharmacy to give more pharmaceutical intervention conducted to more change of prescription. This economic impact was not evaluated also this study was only conducted from the pharmacy's perspective but physicians and nurses also save time.

Disclosure of Interest: None Declared.

\section{PEC06}

Financial inadequacies between medical treatments quotation and medical examinations cost in a French nuclear medicine

J. Descout ${ }^{1, *}$, A. Dumont ${ }^{1}$, V. Tran Quang ${ }^{1}$, P. Hindlet $^{2}$, J.-Y. Devaux ${ }^{1}$

${ }^{1}$ Medecine Nucléaire, ${ }^{2}$ Pharmacy, APHP, Saint Antoine, Paris, France

Background and Objective: In nuclear medicine, diagnostic examinations follow a quotation named CCMA (Common Classification of Medical Acts) which takes into account a technical fixed amount and the radiopharmaceutical drugs cost. Inadequacies between drug prices and the CCMA procedure fees have been identified. In fact, some of these drugs are very expensive and exceed widely the CCMA procedure fees. Thus, the purpose of this study was to assess the inadequacies cost for a year for the nuclear medicine department of Saint-Antoine hospital.

Setting and Method: Inadequacies between the radiopharmaceutical drug cost and the CCMA procedure have been identified according to: the public price, the price directly negotiated by APHP and the procedure rate indicated on the CCMA website. The number of medical examination in 2012 has been extracted from Gera ${ }^{\circledR}$ software.

Main outcome measures: Assessing the financial loss caused by inadequacy between real costs of some examinations and CCMA fees.

Results: Among all the radiopharmaceutical medicines used in 2012 by the nuclear medicine, inadequacy for four drugs got our attention: Datscan $^{\circledR}$ (196 examinations, research of dopaminergic denervation), Octreoscan $^{\circledR}$ (38 examinations, neuroendocrine tumor exploration), 
Adreview ${ }^{\circledR}-\mathrm{I}^{123}$ (15 examinations, medullo-adrenal tracer), Norcholestérol ${ }^{\circledR}-I^{131}$ (5 examinations, adrenocortical tracer). For these 4 drugs, CCMA quotation of the procedure is about 2.4 times bellow the catalogue cost of the product and 2 times bellow the price negotiated by the APHP. Taking into account the number of performed examinations and despite the optimization, the financial loss for the nuclear medicine department in 2012 is $€ 122710$ if we consider the public rates and $€ 109140$ with the rates negotiated by the APHP. Considering the high number of examinations, Datscan ${ }^{\circledR}$ has the biggest impact on the financial loss of the department ( $>€ 90000$ ). In this study, only the costs related to radiopharmaceutical drugs have been analyzed, whereas the CCMA quotation encompasses the totality of the examination and should also take into account the other costs implicated (human ressources, gamma-camera, consumables...). Thus inadequacies should be more important. CCMA rates re-evaluation should allow hospitals to continue these examinations without financial loss. If the prices cannot be renegotiated with pharmaceutical companies, registering these expensive products on the list of extra refunds may be a solution.

Conclusions: Some examinations of nuclear medicine show mismatch between the real cost of the examination itself and the CCMA procedure cost. The financial loss caused by this inadequacy is over $€$ 109,000 for our hospital in 2012 and may lead some hospitals to refuse to practice these examinations to the detriment of the patient. Disclosure of Interest: None Declared.

\section{PEC07}

A study of the efficiency and output of the intravenous mixtures preparation unit

\section{Elberdín Pazos ${ }^{1, *}$, B. Feal Cortizas ${ }^{1}$, M. García Queiruga ${ }^{1}$, L. Ramudo Cela ${ }^{1}$, I. Martín Herranz ${ }^{1}$ \\ ${ }^{1}$ Pharmacy, Complejo Hospitalario Universitario A Coruña, A Coruña, Spain}

Background and Objective: In relation to optimising costs, we set out an analysis of the savings resulting from the centralised preparation of intravenous mixtures (IVM) at the pharmacy service of a hospital with 1,419 beds, as well as the activity in relative value units (RVU) of sterile formulas.

Setting and Method: Prospective longitudinal study over 1 year(2012). Data source: database of IVM preparations, SINFHOS pharmaco-economic management programme, pharmacy service activity and quality panel.

Main outcome measures: In order to calculate the efficiency, we have taken into account the savings resulting from:

1. The centralised preparation of medicines with a high economic impact. Making $100 \%$ use of the contents of the vials for groups of patients.

2. Re-use of returned IVMs

Medicines studied: abatacept, aminoglycosides, liposome and lipid amphotericin B, anidulafungin, caspofungin, daptomycin, anticytomegalovirus immunoglobulin, non specific immunoglobulins, infliximab, lacosamide, tocilizumab, thymoglobulin, vancomycin and voriconazole.

The Catalogue of Products and Invoicing produced by the TECNO group (2009) was used to calculate the RVUs.

Results: 22,003 IVMs were prepared; $58 \%$ aminoglycosides and vancomycin resulting from a recommendation to monitor plasma levels; $9.04 \%$ daptomycin; $7.56 \%$ voriconazole; $5.89 \%$ caspofungin; $3.89 \%$ infliximab.

1. Savings due to centralisation: $€ 403,706$. Main medicines involved: infliximab $45 \%(181,711 €)$; voriconazole $18 \%(73,115 €)$; daptomycin $17 \%(68,480 €)$; caspofungin $16 \%(64.989 €)$.
2. Savings due to re-use: $€ 89,662.9 .9 \%$ returned IVMs, $80.3 \%$ reused. $100 \%$ of the IVMs of infliximab, anticytomegalovirus immunoglobulin and abatacept were re-used, $94 \%$ of caspofungin and daptomycin and $89 \%$ of voriconazole. The medicines that were the least re-used were lipid amphotericin B (45\%) (paediatric doses) and lacosamide (50\%) (small number of patients).

Overall efficiency of IVM preparation unit: $€ 493,368$, representing $0.79 \%$ of the total consumption of medicines for the hospital in this year.

RVUs produced: 388,954 ( $7.63 \%$ of the total for the pharmacy service). Complexity rating for the unit: 13.1 .

Conclusions: The savings made by the IVM preparation unit indicate the high cost effectiveness of pharmacy services, especially in the case of medicines with a high economic impact where the dose is not adjusted to the contents of the vial. The calculation of the RVUs and the complexity rating show the importance of the unit in the activity of the pharmacy service.

Disclosure of Interest: None Declared.

\section{PEC08}

Anti-tumor necrosis factor real-world doses and economic impact in rheumatoid arthritis and ankylosing spondilitis patients: clinical pharmacy data

\section{J. Martinez-Cutillas ${ }^{1, *}$, S. Terradas-campanario ${ }^{2}$,} E. Palacio-Lacambra ${ }^{2}$, C. Alerany-Pardo ${ }^{2}$

${ }^{1}$ Pharmacy, Vall d'Hebron Uniersity Hospital, ${ }^{2}$ Pharmacy, Vall d'Hebron University Hospital, Barcelona, Spain

Background and Objective: Patient individual characteristics may determine clinical response. In clinical practice, anti-tumor necrosis factor (Anti-TNF) treatment patterns are commonly adjusted considering doses and frequency and may vary their efficiency.

Setting and Method: Cross-sectional descriptive study in adult patients who were receiving ADA, ETN, IFX or GOL for at least 6 months the 1st January of 2013 for RA or AS in the Rheumatology Department. Drug patterns prescribed by the rheumatologist were analyze weekly. Escalated and reduced doses were defined as those higher and lower than standard doses respectively. Economic impact of clinical practice with these therapies was evaluated. IFX dose was calculated considering a $-7 \%$ of vial optimization. Day-care costs of administration were not considered.

Main outcome measures: Main outcome measures Patients with rheumatoid arthritis and ankylosing spondylitis treated with each antiTNF are calculated. Anti-TNF dosage, number of patients with escalated or reduced dose and mean percentage of dose increase or reduction vs. recommended with each therapy was analyzed. Associated semi-annual cost savings were estimated based on Spanish public prices including tax.

Results: $\mathbf{5 7 2}$ patients were included in the analysis: 386 patients with RA [ADA $(\mathrm{n}=121)$, ETN $(\mathrm{n}=173)$, IFX $(\mathrm{n}=62)$ and GOL $(\mathrm{n}=23)]$, and 186 patients with AS [ADA $(\mathrm{n}=59)$, ETN $(\mathrm{n}=60)$, IFX $(n=54)$ and GOL $(n=13)]$. Table shows the number of patients with escalated or reduced dosed with each treatment per group of patients. Table also shows dose pattern of use and the associated patient 6 month cost per treatment.

Standard patterns: ETN $50 \mathrm{mg} / \mathrm{week}$, ADA $40 \mathrm{mg} / 2 \mathrm{w}, \mathrm{IFX} * 3 \mathrm{mg} /$ $\mathrm{kg} / 8 \mathrm{w}(\mathrm{RA}) * * 5 \mathrm{mg} / \mathrm{kg} / 8 \mathrm{w}$ (AS), GOL $50 \mathrm{mg} / \mathrm{monthly}$.

Rheumatoid arthritis:

Patients (\%) escalated/reduced: ETN $0 \% / 8.1 \%$; ADA $7.4 \% /$ $14.0 \%$; IFX $30.7 \% / 14.5 \%$; GOL $4.3 \% / 0 \%$.

Pattern (\%) increase/reduction: ETN 0\%/-3.5\%; ADA $6.3 \% /-$ $5.1 \%$; IFX $12.6 \% /-3.7 \%$; GOL $2 \% / 0 \%$ 
Real patient-6 months cost (26wk) (€, \% difference vs. recommended): ETN 5.717,66€ (-3.5\%), ADA 6.509,62€ (+1,2\%), IFX $4.160 €(+9,7 \%)$, GOL $5.250,18 €(+1.6 \%)$

Ankylosing spondilitis:

Patients (\%) escalated/reduced: ETN $0 \% / 6.7 \%$; ADA $8.5 \% /$ $3.4 \%$; IFX $22.2 \% / 22.2 \%$; GOL $23.1 \% / 0 \%$.

Pattern (\%) increase/reduction: ETN $0 \% /-2.5 \%$; ADA $8.5 \% /$ $-1.5 \%$; IFX $4.7 \% /-5.6 \%$; GOL $18.8 \% / 0 \%$

Real patient-6 months cost (26wk) $(€$, \% difference vs. recommended): ETN 5.774,86 € (-2,5\%), ADA 6.878,04 € (+6,9\%), IFX $6.289,92 €(-0,5 \%)$, GOL $6.119,62 €(+18.4 \%)$

$*$ Mean weight $=75.5 \mathrm{~kg}$

Conclusions: Anti-TNF real-world doses shows some differences compared to recommended ones in rheumatology patients. Those differences impact directly on treatment costs and presumably on their efficiency. Under our clinical practice ETN showed the most positive balance in terms of costs reduction between recommended SmPC dose and real dose in clinical practice.

Disclosure of Interest: None Declared.

\section{PEC09}

Change in market of hemodialysis and hemofiltration solutions: clinical and economic impacts

C. Fouche ${ }^{1, *}$, R. Le Guen ${ }^{2}$, S. Thomas ${ }^{1}$, L. Goldwirt ${ }^{2}$, C. Deveaux Chiche ${ }^{1}$, A. Combes ${ }^{2}$, P. Tilleul ${ }^{2}$, N. Poisson ${ }^{1}$

${ }^{1}$ AGEPS, ${ }^{2}$ GH Pitié Salpêtrière, AP-HP, PARIS, France

Background and Objective: Further to expert group "Dialysis" meeting, the pharmaceutical products committee (COMED) decided to replace Prismasol ${ }^{\circledR}$ (with no phosphate) by Phoxilium ${ }^{\circledR}$ ([phosphate $]=1.2 \mathrm{mmol} / \mathrm{L})$. Prismasol ${ }^{\circledR}$ and Phoxilium ${ }^{\circledR}$ solutions are used in continuous renal replacement therapy, especially in hemodialysis and hemofiltration. Dialysis solutions containing phosphate at physiological concentration prevent risk of hypophosphatemia (Broman \& al. 2011) which occurs in $80 \%$ of patients during dialysis because of phosphate's dialysability. Pho$\operatorname{cytan}^{\circledR}$, indicated in phosphate parenterally supply, is used to adjust the concentration of phosphate in dialysis bags. A centralized market is signed for our whole hospital structure composed of 37 hospitals. The market has been changed since May 2012. This study aims at assessing the impact of this modification on clinical practices in regard to hypophosphatemia risk for dialysis patients. It also aims at evaluating economic effects at 3 levels: the whole hospital structure, the hospital, the intensive care unit (ICU) setting.

Setting and Method: Consumptions of Prismasol ${ }^{\circledR}$, Phoxilium ${ }^{\circledR}$ and Phocytan ${ }^{\circledR}$ were observed on the stock control software (SAP and Copilote) at every level. Clinical practitioner opinions were collected to assess modifications in clinical practices.

Results: Regarding the whole hospital structure, since Prismasol ${ }^{\circledR}$ substitution by Phoxilium ${ }^{\circledR}$, the consumption of these solutions has remained stable (about 2150 bags monthly). Phoxilium ${ }^{\circledR}$ is more expansive than Prismasol ${ }^{\circledR}(+1.50 €$ by bag $)$, which generates an overspend by $19 \%$ monthly ( $+3300 €$ monthly). As the same time, Phocytan ${ }^{\circledR}$ consumption has been reduced by $15 \%\left(\begin{array}{l}-4 \\ 870 €\end{array}\right.$ monthly) since the market changed. On the whole, a reduction by 1 $570 €$ monthly is observed.

Regarding the hospital, no variation is observed concerning the consumption of these solutions (about 1100 bags monthly). However, Phocytan ${ }^{\circledR}$ consumption has decreased by $28 \%$ since the market changed.

Regarding the ICU, Prismasol ${ }^{\circledR}$ and Phoxilium ${ }^{\circledR}$ were principally used in this unit in 2012 (60\% of the hospital consumptions). That's why the ICU has been chosen for this study. Considering the augmentation of dialysis activity in the ICU, monthly consumptions are up $54 \%$ for this couple of products $(+2344 €$ monthly): $29 \%$ due to the growing activity and $25 \%$ due to the difference in the unit price between these two products. As for Phocytan ${ }^{\circledR}$, consumptions decreased by $72 \%$ ( $-539 €$ monthly). The use of these products in the ICU has increased spendings up $35 \%$ (+1 $806 €$ monthly) since the market changed.

Conclusions: Changing from Prismasol ${ }^{\circledR}$ to Phoxilium ${ }^{\circledR}$ implies clinical practices modification in the intensive care unit with: a diminution of mistake risk in phosphate supplying, a diminution of risk in regard to bags manipulation, a moderate economic impact at local level but profitable for the whole hospital structure.

Disclosure of Interest: None Declared.

\section{PEC10}

What would you do without Medicine information? A service evaluation to investigate the costs avoided by the provision of medicine information service

N. Alkhaldi ${ }^{1, *}$, D. J. Wright ${ }^{1}$, R. Little ${ }^{2,3}$, L. Morgan ${ }^{1}$, T. House ${ }^{4}$

${ }^{1}$ Pharmacy practice deartment, ${ }^{2}$ Medicine, ${ }^{3}$ Health Economics Consulting, Norwich Medical School, University of East Anglia, Norwich, ${ }^{4}$ Medicine information service, Cambridge University Hospital NHS Foundation Trust, Cambridge, United Kingdom

Background and Objective: To calculate the potential costs of doctors answering their own medicine information (MI) queries in the absence of MI services to enable a cost-effectiveness model for a hospital based MI service to be developed.

Setting and Method: A telephone survey over six months in a large teaching hospital in England; after appropriate ethical approval has received doctors phoned MI centre with queries related to specific patient and agreed verbally to participate in the study were asked what they would do in the absence of MI service and were estimated how long they would spend in answering process. Data collection form were designed and were tested, during pilot study, to captures the data required to estimate the time taken of doctors answering their MI queries in the absence of the MI service. The costs of both doctors and MI staff providing MI answering service were calculated using the published salary survey on the unit costs (PSSU) of health and social care 2011. Mean and $95 \%$ confident intervals (CI) were used to describe results.

Main outcome measures: Estimated cost of doctors answering the MI queries themselves.

Results: Of 36 doctors (consultants $6(17 \%)$, registrars $12(33 \%)$, junior doctors $18(50 \%)), 61 \%$ stated to undertake the research process by themselves. Doctors suggested time taken answering MI queries ranged from 1 to 450 min, mean $(95 \% \mathrm{CI})$ of 53 (27 to 78.8 ), with costs ranged from $£ 1.12$ to $£ 499.71$, mean (95\% CI) of 83.8 (47.9 to 119.7). MI staffs spend from 1 to $488 \mathrm{~min}$, mean $95 \%$ CI of 74 (40.1 to 107.9), with costs ranged from $£ 1.22$ to £549.0, mean $95 \%$ CI (39.9 to 124.1). 7 (19\%) of doctors were $100 \%$ confident answering medicine related questions, $5(14 \%)$ were $90 \%$ confident, $16(44 \%)$ were $50 \%$ confident, 6 (17) were $20 \%$ and $2(6 \%)$ were completely had no confidence with their answers.

Conclusions: Doctors calculated costs mainly depend on their estimation of time required to answer MI queries. Although doctors predicted costs were almost the same as MI staff costs, the effectiveness appears to be less. However, this study would support the development of the cost-effectiveness model.

Disclosure of Interest: None Declared. 


\section{Pharmacotherapy}

\section{PT17}

Prevalence of adverse reactions in patients treated with non-ionic iodinated contrast

J. Alonso Herreros ${ }^{1, *}$, C. Murcia-Murcia ${ }^{1}$, A. González-Cuello², J. Abellon Ruiz ${ }^{3}$

${ }^{1}$ Pharmacy, Hospital General Universitario Reina Sofia (Murcia), Murcia, ${ }^{2}$ Pharmacology, Universidad de Murcia, Murcia, ${ }^{3}$ Pharmacy, Hospital Los Arcos Mar Menor, Santiago De La Ribera, Spain

Background and Objective: The Contrast-Induced Nephropathy (CIN) (relative elevation of serum creatinine greater than or equal to $25 \%$ during the $48 \mathrm{~h}$ after the administration) is a major problem due to: a) the frequency of imaging studies made at present; b) it can affect $50 \%$ of patients with some risk factors (diabetes, age $>75$, renal-disease, cirrhosis, or nephrotoxic drugs); and c) it can favour progressive renal damage and mortality at one year.

At risk patients prevention, has been suggested by several guidelines, including infusions of sodium bicarbonate, and $\mathrm{N}$-acetylcysteine.

The aim of this study was to evaluate the outcomes of the implemented local guidelines for prevention of CIN with acetylcysteine (1,200 mg, bid for two days) and bicarbonate (500 $\mathrm{ml} \mathrm{1/6} \mathrm{M} \mathrm{IV)}$ in our teaching hospital.

Setting and Method: We performed an observational retrospective study. The population was 5,142 patients treated with contrast media in our teaching hospital in 2012. A sample size of 115 risk patients was calculated (confidence level of $90 \%$, error of $7.5 \%$, frequency of CIN equal $40 \%$ ).

The analysis of the results was performed by calculating odds ratios and the Fisher's test.

Main outcome measures: Analyzing the percentage of patients at risk who have received prophylaxis for CIN according to the local guidelines. Calculating the percentage of patients who have suffered CIN despite of having been administered the prophylaxis. Analyzing the effectiveness of the guidelines to prevent CIN

Results: In the group of patients who received prophylaxis $(84 \%)$, only $3.12 \%$ suffered from CIN. While in the group who did not receive prophylaxis, $26.32 \%$ suffered from CIN. The Fisher's test shows a statistically significant difference $(p<0.01)$. The odds ratio $(\mathrm{OR}<1)$ indicated also that prophylaxis is a protective factor for the CIN.

Conclusions: Prophylaxis with acetylcysteine $(1,200 \mathrm{mg}$, bid for two days) and bicarbonate (500 $\mathrm{ml} 1 / 6 \mathrm{M} \mathrm{IV})$ is effective in reducing the Contrast-Induced Nephropathy in at-risk patients.

Disclosure of Interest: None Declared.

\section{PT20}

Evaluation of restricted antibiotic use in a hospital in Romania

P. Tarcea Bizo ${ }^{1, *}$, D. Dumitras ${ }^{2}$, A. Popa ${ }^{1}$

${ }^{1}$ Clinical Pharmacy, University of Medicine and Pharmacy "Iuliu Hatieganu", Faculty of Pharmacy, ${ }^{2}$ Management and Econometrics, University of Agricultural Science and Veterinary Medicine, Cluj Napoca, Romania

Background and Objective: To evaluate the use of restricted antibiotics in an academic tertiary hospital in Cluj-Napoca, Romania
Setting and Method: Retrospective evaluation of the use of restricted antibiotics issued based on the antibiotics formularies for the year 2012. International Therapy Guidelines and the Summary of product characteristics were used for the evaluation. Descriptive statistics was performed to summarize the results. Multiple logistic regression analysis was also used to identify independent risk factors for the inappropriate prescription of antibiotics.

Main outcome measures: The appropriateness of the use of 11 antibiotics was verified, according to 3 main criteria: proper dose, appropriate indication (type of treatment, localization and type of infection) and appropriate duration of treatment.

Results: 664 prescribing formularies for antibiotics were analyzed, of these 319 were from the intensive care unit $(48.04 \%)$. The most prescribed antibiotics were: Vancomycin (171 prescriptions, $25.75 \%$ ), Imipenem (151 prescriptions, $22.74 \%$ ) and Meropenem (116 prescriptions, $17.47 \%$ ). Of these $285(42.92 \%)$ are considered inappropriate. Of the prescriptions for Vancomycin $49.71 \%$ were considered inappropriate, $46.55 \%$ were considered inappropriate for Meropenem and $44.06 \%$ for Imipenem. Of the total 285 prescriptions deemed as inappropriate, for $49.82 \%$ the dose was incorrect $20 \%$ were inadequate in terms of treatment duration and $15.44 \%$ were wrongly indicated. Inappropriate use was significantly higher among empirical prescriptions than the documented ones $(69.75 \%$ vs. $30.25 \%, p<0.001)$. Restricted antibiotics are frequently prescribed inappropriate, without guideline indication for empirical use. Antibiotic prophylaxis also raises issues: the dosage and duration are increased, and in most cases restricted antibiotics are not indicated in surgery prophylaxis. Multiple stepwise logistic regression identified that the duration of the treatment was significant for inappropriate antibiotic use $(p<0.05)$. The risk of inappropriate use in the case of empirical prescriptions is higher than for documented prescriptions $(\mathrm{OR}=5.78, p<0.001, \mathrm{CI}=$ 3.65-9.15).

Conclusions: There are a large number of inappropriate prescriptions which show the need to intensify the control of the use of restricted antibiotics. Other favorable factors such as the implementation of drug formularies in hospitals and the involvement of the clinical pharmacist in order to ensure rational antibiotic therapy, may improve the quality of patient care.

Disclosure of Interest: None Declared.

\section{PT21}

Certolizumab in Crohn's disease after failure of two other antiTNF antibodies.

M. Suarez Santamaría ${ }^{1, *}$, D. Perez Parente ${ }^{1}$, S. Sanmartin Alvarez ${ }^{2}$, J. M. Castro Dominguez ${ }^{1}$, S. Suarez Ordoñez ${ }^{3}$, N. Martinez Lopez de Castro ${ }^{1}$, G. Piñeiro Corrales ${ }^{1}$

${ }^{1}$ Pharmacy, Hospital Meixoeiro, Vigo, ${ }^{2}$ Pharmacy, Susana.San.Martin.Alvarez@ sergas.e, vigo, ${ }^{3} \mathrm{Hematology,}$ Hospital Meixoeiro, Vigo, Spain

Background and Objective: Crohn's disease (CD) is a chronic inflammatory disorder of the gastrointestinal tract. Although mild disease can be treated with 5-aminosalicylates, many patients eventually require corticosteroids to control symptoms. Many patients are unable to discontinue corticosteroid therapy without disease exacerbation. The purine antimetabolites and methotrexate are frequently prescribed for patients who are resistant to or dependent on corticosteroids; however, these drugs have a slow onset of action and clinical remission rates of about $40 \%$. Certolizumab pegol is a humanized 
monoclonal antibody Fab fragment linked to polyethylene glycol that neutralizes tumor necrosis factor. It received approval from FDA in 2008 for treatment and maintenance of response in adults with moderate to severe $\mathrm{CD}$ who had an inadequate response to conventional therapy, but it has not been approved by the European Medicines Agency and is therefore not widely available in Europe. We describe the case of Crohn's disease refractory to other treatments which was treated with certolizumab pegol.

Setting and Method: A 29 years old women presenting CD refractory to conventional therapy since last 12 years. She was treated with azathioprine and methotrexate (failed) and infliximab (serious adverse transfusion reaction at the fourth dose). Then she was treated with adalimumab, responding for 12 months but gradually losing effectiveness. Increasing doses produced no response, then she underwent surgical. After surgery she was treated with prophylactic therapy with azathioprine and remained asymptomatic for 10 months, but with persistent elevation of acute-phase reactants. The absence of efficacy of azathioprine and the appearance of recurrent urinary tract infections, the patient was treated with adalimumab $40 \mathrm{mg}$ every 2 weeks. The dose was increased to $80 \mathrm{mg}$ by progressive loss of efficacy but this therapy was not effective. However, the treatment with ustekimumab as compassionate use was authorised. First response was to excellent, but at 7-8 months abdominal pain and diarrhea was developed, and the treatment was discontinued. Afterwards, patient was treated with certolizumab (induction dose $400 \mathrm{mg} \mathrm{0,2}$ and 6 weeks and maintenance dose every 8 weeks) that was approved for compassionate use by the national regulatory agency on drugs.

Results: Once started, all the acute-phase reactants decreased to normal levels. In the first month the patient remained asymptomatic that allowed to reduce the dose of prednisone to $10 \mathrm{mg} /$ day. Unfortunately, after reducing the dose of steroids, the patient had a worsening of symptoms and progression of the radiologic findings. The patient is currently waiting for

Conclusions: Blocking anti-TNF in this case seems to be ineffective. We suggest that other biological drugs with different mechanism of action could be considered to treat this case.

Disclosure of Interest: None Declared

\section{PT22}

\section{Daytime Intragastric pH With Esomeprazole 20 mg vs OTC} Proton Pump Inhibitors

\section{T. Lind ${ }^{1, *}$, P. Katz ${ }^{2}$, J. Dent ${ }^{3}$, P. Kahrilas ${ }^{4}$, B. Traxler ${ }^{5}$,} K. Röhss ${ }^{6}$, D. Johnson ${ }^{7}$

${ }^{1}$ Astra Zeneca, Mölndal, Sweden, ${ }^{2}$ Albert Einstein Medical Center, Philadelphia, United States, ${ }^{3}$ Royal Adelaide Hospital, Adelaide, Australia, ${ }^{4}$ Northwestern University, Chicago, ${ }^{5}$ AstraZeneca, Wilmington, United States, ${ }^{6}$ AstraZeneca, Mölndal, Sweden, ${ }^{7}$ Eastern Virginia Medical School, Norfolk, United States

Background and Objective: In patients with untreated gastroesophageal reflux disease (GERD), most symptoms occur during waking hours. We compared intragastric $\mathrm{pH}$ in the 14-hour daytime period for esomeprazole $20 \mathrm{mg}$ and over-the-counter (OTC) doses of other proton-pump inhibitors (PPIs) using data from published studies. $^{1-4}$

Setting and Method: In one double-blind ${ }^{1}$ and three open-label ${ }^{2-4}$ randomized, crossover studies, intragastric $\mathrm{pH}$ was monitored for $24 \mathrm{~h}$ on treatment day 5 . In this analysis, acid control with OTC PPIs was reassessed for the 14-h daytime period (awake time). Mean percentage of awake time with $\mathrm{pH}>4$ was estimated for each study using a linear mixed-effect model (treatment, treatment period, and treatment sequence as fixed effects; subject nested with treatment sequence as a random effect). Using this model, geometric mean ratios (GMR) were estimated based on log-transformed individual values.

Main outcome measures: Mean percentage of awake time with $\mathrm{pH}>4$ for esomeprazole $20 \mathrm{mg}$ versus OTC PPIs.

Results: In the double-blind study of GERD patients $(\mathrm{N}=36)$, mean (95\% CI) percentage of awake time with $\mathrm{pH}>4$ was $61.9 \%(53.9 \%$ $>69.9 \%)$ with esomeprazole $20 \mathrm{mg}$ versus $51.7 \%(43.7 \%>59.7 \%)$ with omeprazole $20 \mathrm{mg}$ (GMR [95\% CI] $=1.45 \quad[1.14-1.85]$; $P<.01)$. In an open-label GERD study $(\mathrm{N}=38)$, mean $(95 \% \mathrm{CI})$ percentage of awake time with $\mathrm{pH}>4$ was $55.2 \%(49.2 \%>61.1 \%)$ with esomeprazole $20 \mathrm{mg}$ versus $28.0 \%(22.0 \%>34.0 \%)$ with pantoprazole $20 \mathrm{mg}$ (GMR [95 \% CI] $=2.50$ [2.01-3.11]; $P<.0001)$. Two studies of healthy volunteers $(\mathrm{N}=37 ; \mathrm{N}=26)$ compared esomeprazole $20 \mathrm{mg}$ with lansoprazole $15 \mathrm{mg}$. Mean (95\% CI) awake time with $\mathrm{pH}>4$ for esomeprazole was $51.2 \%(45.6 \%>56.8 \%)$ and $55.8 \%(43.5 \%>68.0 \%)$; for lansoprazole, it was $31.5 \%(25.9 \%$ $>37.0 \%)$ and $45.2 \%(33.0 \%>57.5 \%)$. The GMR for esomeprazole/ lansoprazole was $1.69(95 \%$ CI $1.46-1.97 ; P<.0001)$ and $1.89(95 \%$ CI 1.05-3.37; $P<.05$ ).

Conclusions: During the period of daytime food-stimulated acid secretion and postprandial reflux, esomeprazole $20 \mathrm{mg}$ provided acid control for a significantly greater portion of the awake time vs other PPIs at OTC doses.

\section{References:}

1. Lind T, et al. Aliment Pharmacol Ther. 2000;14(7):861-7.

2. Wilder-Smith C, et al. Clin Drug Invest. 2008;28(6):333-43.

3. Wilder-Smith C, et al. Scand J Gastroenterol. 2007;42(2): 157-64.

4. Röhss K, et al. Clin Drug Invest. 2004;24(1):1-7.

Disclosure of Interest: None Declared

\section{PT23}

Assessment of professional practices regarding the prescription of proton pump inhibitor

J. Lizon ${ }^{1, *}$, L. Huynh-Lefeuvre ${ }^{1}$, G. Grangier ${ }^{1}$, M. Temporelli ${ }^{1}$, C. Hofmann ${ }^{1}$

${ }^{1}$ Pharmacie, Hôpital d'Instruction des Armées LEGOUEST, METZ, France

Background and Objective: Proton pump inhibitors (PPI) are a widely prescribed therapeutic class. PPIs are highly used despite recommendations on the appropriate use of these medicines. Thus, numerous prescriptions are made in clinical situations outside marketing authorization as they are very well tolerated.

The aim is to conduct an assessment of professional practices regarding the use of PPIs on all hospital services. The purpose of this study is to analyse the methods of PPI prescription and their relevance. Setting and Method: A multidisciplinary committee was initially formed, with several members: physicians of the gastroenterology department, pharmacists and a pharmacy intern. Meetings have been held in order to determine the study approach and schedule, as well as the way forward. A guideline (with directions and dosage) based on the French Health Authority's recommendations was produced as well as a data collection sheet, which were validated by the committee members.

The included patients have been those for whom a treatment through PPI was orally or intravenously prescribed throughout the study-i.e. a total of ten weeks.

Results: During the study, 331 PPI prescriptions have been analysed. The population being studied was predominantly men $(55 \%$ versus 
$45 \%)$ and a group over 60 years old $(69.1 \%)$. The PPI was orally prescribed in 276 cases $(83.4 \%)$. The treatment was given as follows: 318 with 1/day (96\%), 11 with 2/day (3.32\%), 1 with continuous use $(0.3 \%)$ and 1 with $3 /$ day $(0.3 \%)$. Out of the 11 cases with $2 /$ day, 6 $(54.54 \%)$ complied with the guideline and $5(45.45 \%)$ did not. As regards the choice for the IV route, 46 out of 55 prescriptions were compliant $(83.6 \%)$. Finally, as far as the PPI prescription is concerned, 159 prescriptions were guideline compliant (47.96\%), 103 were guideline non-compliant $(31.2 \%)$ and 69 of which indication was unknown to the prescriber $(20.84 \%)$. Among the non-compliant ones, 8 concerned the dosage $(7.76 \%)$ and 95 the indication $(92.24 \%)$.

Conclusions: Of the 9 prescriptions that are non-compliant as regards the choice of the IV route, oral or enteral administration could have been used. Among the 95 prescriptions that are non-compliant with respect to the PPI indication, $51(53.68 \%)$ had the following indication: "Prevention of stress ulcer" (except intensive care). The results were presented to the hospital medical commission (CME Commission médicale d'établissement). A guideline will be prepared in order to promote appropriate use of PPI in the hospital setting. It is important to raise awareness among the prescribers towards PPI adverse effects and, in particular, the risk of bacterial pneumonia caused by decreased intragastric acidity.

Disclosure of Interest: None Declared.

\section{PT25}

Identifying inappropriate anticholinergic prescribing in the elderly: a systematic review

\section{S. Al Shemeili ${ }^{1, *}$, S. Klein ${ }^{2}$, A. Strath ${ }^{1}$, D. Stewart ${ }^{1}$ \\ ${ }^{1}$ School of Pharmacy and Life Sciences, ${ }^{2}$ Institute for Health and Welfare Research, Robert Gordon University, Aberdeen, United Kingdom}

Background and Objective: Drugs with anticholinergic properties are widely used in elderly patients despite their well recognised adverse effect profiles. The objective of this research was to undertake a systematic review of the published literature relating to the application of criteria or tools to identify and resolve potentially inappropriate prescribing of anticholinergics in elderly patients.

Setting and Method: A systematic review protocol was developed. The search string included: anticholinergic, cholinergic antagonist; elderly, old age; health professional, doctor, nurse, pharmacist, physician; guideline, tool, criteria, START, STOPP, Beers, listed in the title, keywords, abstract or text. Boolean operators, truncation and wildcards were applied. The following databases were searched: Medline; Cumulative Index of Nursing and Allied Health Literature; International Pharmaceutical Abstracts; PsycInfo; and Cochrane Database of Systematic Reviews. Studies published in English between 2000 and 2013 were included. Each paper was assessed by two reviewers for methodological quality prior to inclusion in the review. Data were extracted and studies critically appraised using standardized tools.

Main outcome measures: Number of studies meeting review criteria, location, setting, tools or guidelines applied, potentially inappropriate prescribing identified, impact on patient care.

Results: Database searching identified 577 studies, 27 of which were included in the review. All studies used an observational, cross-sectional type design. Studies were reported from Europe (10), the Americas (9), Australia and Oceania (4) and Asia (4). Settings were general practice (14), hospital (6), care homes (4) and not reported (3). Criteria and tools reported were Beers Criteria (8), Drug Burden Index Score (8), Anticholinergic Cognitive Burden Scale (4), Anticholinergic Risk Scale (3), STOPP/START criteria (3) and 'Australian' Criteria (2). Several studies used more than one criteria/ tool. While the application of these identified potentially inappropriate prescribing of anticholinergics, no studies reported subsequent changes in prescribing and impact on patient care.

Conclusions: While several generic or anticholinergic specific criteria or tools have been applied by health professionals in different healthcare settings to identify potentially inappropriate prescribing of anticholinergics in elderly patients, there is a lack of data surrounding impact on prescribing and consequent patient outcomes. These issues should be given consideration in further research.

Disclosure of Interest: None Declared.

\section{PT26}

Potentially inappropriate prescribing in older patients: a meta-synthesis

\section{S. Cullinan ${ }^{1, *}$, D. O'Mahony ${ }^{2}$, A. Flemming ${ }^{1}$, S. Byrne ${ }^{1}$}

${ }^{1}$ School of Pharmacy, University College Cork, ${ }^{2}$ Department of Geriatric Medicine, Cork University Hospital, Cork, Ireland

Background and Objective: Potentially inappropriate prescribing (PIP) is commonly seen amongst the older population. The prevalence of PIP has been estimated to be $58 \%$ in the secondary care setting (1). Quantitative work such as this has highlighted the issue and attracted attention. However, less attention has been focused on why it is happening.

This paper's aim was to synthesise qualitative studies that explore PIP in older patients, in an effort to understand why it happens and generate new theory that can guide future intervention studies and progress the field. Qualitative work in general is lacking in this area. The studies that do exist have never been brought together in a systematic synthesis such as this.

Setting and Method: Literature review. Three databases were systematically searched: PubMed, EMBASE and Web of Knowledge. No date restrictions were applied. Key words searched were: Qualitative AND (Inappropriate* OR Appropriat* OR Safe) AND (Elderly OR Aged OR Geriatric* OR Old*) AND Prescri*. Reference lists of papers located were then searched for other suitable papers that should be included. Using meta-ethnography (2), the papers were translated into each other in a reciprocal translation by two researchers, and reinterpreted to generate new all-encompassing theory in a line of argument synthesis.

Main outcome measures: Common themes throughout the papers suggesting reasons for PIP occurring.

Results: After application of inclusion/exclusion criteria, 7 papers were deemed relevant. Four key concepts were identified as being causal factors in PIP: (1) the need to please the patient, (2) feeling forced to prescribe, (3) experience VS guidelines and (4) fear. These were re-interpreted in a line of argument synthesis which suggests that doctors are rendered powerless to prescribe appropriately due to a combination of factors.

Conclusions: PIP in older patients is a result of many factors combining to render the prescriber powerless to prescribe in an appropriate manner. This power needs to be re-acquired through better communication, education and collaboration in order for the issue to be resolved. The problem is not a lack of guidelines. It is an abundance of barriers to implementing these guidelines, which need to be systematically removed through suitable intervention studies.

\section{References}

1. Gallagher P, Lang PO, Cherubini A, Topinkova E, Cruz-Jentoft A, Montero Errasquin B, et al. Prevalence of potentially inappropriate prescribing in an acutely ill population of older patients admitted to six European hospitals. Eur J Clin Pharmacol. 2011;67(11):117588 . 
2. Noblit GW, Hare RD. Meta-Ethnography: synthesising qualitative studies. London: Sage. 1988.

Disclosure of Interest: None Declared.

\section{PT27}

Effectiveness and safety of sorafenib in thyroid cancer

M. Mateos Salvador ${ }^{1, *}$, T. Calleja Chuclá ${ }^{1}$, F. Busto Fernández ${ }^{1}$, C. Vilaboa Pedrosa ${ }^{1}$, C. Martínez Roca ${ }^{1}$, P. Yáñez Gómez ${ }^{1}$, I. Martín Herranz ${ }^{1}$

${ }^{1}$ Pharmacy, Complejo Hospitalario Universitario De A Coruña, A Coruña, Spain

Background and Objective: To describe the effectiveness and toxicity of sorafenib in patients diagnosed with thyroid cancer.

Setting and Method: Retrospective observational study in a tertiary referral hospital. Period: 2009-2013. $100 \%$ of patients with thyroid cancer treated with sorafenib. Data gathered: gender, age, cellular classification of thyroid cancer, year of diagnosis, prior treatment, posology of sorafenib, duration of treatment, tumour response rate and adverse reactions (AR).

Main outcome measures: The demographic and clinical characteristics of the patients were analysed, the response rate [stable disease (SD), progressive disease (PD), partial response (SR)] (response evaluation criteria in solid tumours-RECIST) and toxicity (common terminology criteria for adverse events-CTCAE).

Results: Total number of patients: 5 ( 2 male). Mean age: 71 years (range 44-77 years). Diagnostic classification: 2 papillary thyroid, 2 follicular (1 Hürthle cell carcinoma) and 1 anaplastic. Average time of diagnosis: 7 years (mean) (range $0-11$ years). $100 \%$ of patients in stages III-IV, having previously received multiple types of treatment: total thyroidectomy, radiotherapy with iodine-131, palliative radiotherapy.

Initial dose in all cases: $400 \mathrm{mg} / 12 \mathrm{~h}(2$ patients required an adjustment of the dose to $200 \mathrm{mg} / 12 \mathrm{~h}$ due to adverse drug reactions). Length of treatment: 120 days in 4 patients (mean) (range 36-202 days) (one patient currently undergoing treatment). Suspension of treatment: death (1 patient), AR (1 patient), PD (1 patient), unknown (1 patient). Tumour response: SD 2 patients, PD 2 patients. 1 patient currently undergoing treatment, without data. One of the patients died from papillary cancer with metastasis in the lung. The duration of the response could not be evaluated due to the retrospective nature of the study. AR: After 15 days 1 patient developed a rash, treatment was suspended and restarted at half the dose for 15 days, then continuing with the initial dose. Another patient presented a skin reaction after 3 months of treatment, G2 palmar-plantar erythrodysesthesia and G2 alopecia, leading to dose reduction to $200 \mathrm{mg} / 12 \mathrm{~h}$.

Conclusions: Sorafenib can be considered as an effective option in the treatment of advanced thyroid cancer, in line with the data obtained in the literature. It has an acceptable profile in terms of its safety and tolerance.

Disclosure of Interest: None Declared.

\section{PT28}

Adverse drug reactions: a nursing perspective

\section{P. Štrbová ${ }^{1, *}$, L. Mazalováa ${ }^{2}$, K. Urbánek ${ }^{1}$}

${ }^{1}$ Department of Pharmacology, Faculty of Medicine and Dentistry, Palacký University, Olomouc, ${ }^{2}$ Department of Nursing, Faculty of Health Sciences, Palacký University, Olomouc, Olomouc, Czech Republic
Background and Objective: Reducing the incidence of adverse drug reactions (ADRs) and their early detection is strongly dependent on the knowledge of health care professionals. Nurses are an integral part of the multidisciplinary programs aimed at the detection and assessment of ADRs in hospitals. A large proportion of ADRs occur in geriatric patients, so nurses should be especially educated in the characteristics of ADRs in these patients.

Setting and Method: The study was aimed at nurses' knowledge of adverse drug reactions and their personal experience with adverse drug reactions. The data were obtained by the questionnaire containing 18 questions. The survey was performed among nurses with at least one year of praxis at the geriatric and internal medicine departments of University Hospital in Olomouc and Jeseník Hospital, Czech Republic.

Main outcome measures: Nurses' knowledge and experience with ADRs and proper medication use in elderly patients.

Results: Among 50 respondents, 32 had $(64 \%)$ secondary education, 10 $(20 \%)$ postsecondary education and $8(16 \%)$ university (baccalaureate) education. Less than $80 \%$ of them had experience with ADRs in last month of their praxis and $12 \%$ detected any drug administration error. Most frequently claimed ADRs were skin reactions, bleeding and upper GIT symptoms. Mentioned medication errors were wrong dosage or administration of wrong drug. Vast majority, $72 \%$ (36) of asked nurses, were familiar with correct procedure for ADR reporting. Approximately $28 \%$ were able to indicate the risk drug groups for geriatric patients. A total of $88 \%$ of nurses participated in patients' education in the proper medication use.

Conclusions: Nurses knowledge of ADRs was found to be quite satisfactory; however, they could be improved in some areas, mainly in specifics of medication administration in the elderly. Extension of knowledge on ADRs could be achieved by intensification of both undergraduate and graduate nurse education and carrying out of training. Collaboration with clinical pharmacists or pharmacologists in graduate nurse education is highly advisable.

Disclosure of Interest: None Declared.

\section{PT29}

Our experience with DPP-4 inhibitor therapy in diabetologist's ambulance

D. Rubintova ${ }^{1, *}$, J. Lacka ${ }^{2}$, T. Foltanova ${ }^{1}$, S. Jankyova ${ }^{1}$

${ }^{1}$ Faculty of Pharmacy, Bratislava, ${ }^{2}$ Diabetologist's ambulance, Trnava, Slovakia

Background and Objective: The aim of this study was to evaluate DPP-4i therapy and to compare the impact of treatment with linagliptin, vildagliptin and saxagliptin on fasting glycaemia and HbA1c. Setting and Method: Retrospective analysis of the 84 diabetic patients data were evaluated by ANOVA in SPSS. All patients were treated with metformin/sulfonylurea derivative \pm DPP4i. They were divided according length of DPP-4i treatment (newly added "A"; treated $>1.5$ year "B"; without DPP-4i "C") and type of DPP-4i (vildagliptin/linagliptin/sitagliptin/saxagliptin).

Main outcome measures: Glycaemia and HbAlc levels and percentage of patients with proper compensation of DM2.

Results: Glycaemia decreased in the group "A" (8.77 vs. $8.37 \pm 0.52 \mathrm{mmol} / \mathrm{l})$, while in the group "C" glycaemia increased (7.96 vs. $8.08 \pm 0.46 \mathrm{mmol} / \mathrm{l})$. Increased glycaemic levels were also shown in the group "B" (7.17 vs. $7.74 \pm 0.42 \mathrm{mmol} / \mathrm{l})$. Together $47.94 \%$ of the patients were treated with vildagliptin, $39.72 \%$ linagliptin, $6.85 \%$ sitagliptin and $5.48 \%$ saxagliptin. The most effective decrease of glycaemia and $\mathrm{HbA1c}$ was shown by saxagliptin, that significantly decreased HbA1c.

Conclusions: Our preliminary results showed the increase in glycaemia and HbA1c in the patients on a long-term therapy with DPP-4i. 
The reason for this could be the progression of diabetes; however, due to the lack of clinical studies, we cannot exclude the tolerance formation, neither. For the setting of the proper reason, long-term data collection is needed as well as the extension of the patients group. The patients using saxagliptin had the best compensation of glycaemia and HbA1c that reached less than $<7 \%$, the patients treated with vildagliptin had decreased levels of HbA1c, however it was higher than $7 \%$. The mildest decrease of HbAlc was shown in the patients that were administered linagliptin, their glycaemic level was increased as well. The limitation of this study is the mean amount of the patients in which the DPP-4i was added to therapy.

Disclosure of Interest: None Declared.

\section{PT30}

Is safety monitoring of retinoids for the systemic treatment of acne in clinical practice sufficient?

\section{Kakosova ${ }^{1, *}$, R. Kamenska ${ }^{2}$, T. Magalova ${ }^{2}$, T. Foltanova ${ }^{3}$, M. Goboova ${ }^{4}$}

${ }^{1}$ Pharmacy Department, Childen's University Hospital, Bratislava, Slovakia, ${ }^{2}$ Drug Safety and Clinical Trials Section, State Institute for Drug Control, ${ }^{3}$ Department of Pharmacology and Toxicology, Faculty of Pharmacy, Comenius University, Bratislava, ${ }^{4}$ Department of Internal Medicine and Clinical Pharmacology, Teaching Hospital, Nitra, Slovakia

Background and Objective: Isotretinoin (13-cis-retinoic acid) is a retinoid compound and derivate of vitamin A. It is used for the systemic treatment of severe forms of acne. Like all retinoids, isotretinoin is teratogen and is absolutely contraindicated during pregnancy to avoid congenital defects. It should only be prescribed to women of childbearing potential under strict pregnancy prevention measures supported by a Pregnancy Prevention Program (EMEA Oct. 2003). The first oral isotretinoin product Roaccutane was registered in all EU Member States, except Sweden, from 1983. Now generics of isotretinoin (Aknenormin, Curacné) are available on our market.

Setting and Method: Presented are results of retrospective study of all isotretinoin spontaneous adverse drug reactions (ADRs) reported in Slovakia and pregnancy exposure to isotretinoin during treatment of acne. Data, from years 2006 to 2013, were collected from database of the State Institute for Drug Control.

Main outcome measures: Type, frequency and seriousness of ADRs due to exposure to isotretinoin are evaluated.

Results: During the followed period 12 patients with ADRs (aged 17-34 years, 6 males and 6 females) were reported. The serious ADRs of Roaccutane were mainly: depression, suicidal ideation, haematological aggravation, gynaecomastia, nervousness, and unintentional weight loss. Its non-serious ADRs were exanthema maculosis and angioedema of face. The serious ADRs of Acnenormin were subabdominal pain and reduced ability to move legs, and its non-serious ADRs were: swelling of ankle, arthralgia, myalgia, chest pain, anxiety, and weeping. The non-serious ADR of Curacné was dyspnoea. Moreover, during the years 2007-2009, 6 pregnancies (aged 16-23 years) were exposured to isotretinoin: Roaccutane-4 cases, Aknenormin-1 (induced abortion), and undefined isotretinoin-1.

Conclusions: While remaining ADRs are described relatively well, reports concerning isotretinoin exposure of pregnant women are less exhaustive. Information about incidence of spontaneous abortion, malformation of the foetus and on further development of the born children is missing.

Disclosure of Interest: None Declared.
PT31

Management of patients receiving paliperidone palmitate (drug for long acting injection) in a psychiatric hospital: State of the first months of clinical practice

S. Taurin ${ }^{1, *}$, B. Langree ${ }^{1}$, A. Laugueux ${ }^{1}$, N. Marie ${ }^{1}$, G. Burgot ${ }^{1}$ and Centre Hospitalier Guillaume Régnier (CHGR), Rennes, France

${ }^{1}$ Pharmacie, Centre Hospitalier Guillaume Regnier, Rennes, France

Background and Objective: Paliperidone palmitate (PLP) or 9 Hydroxyrisperidone, the active metabolite or risperidone has been approved in France since February 2013. It's an atypical antipsychotic with several advantages compared to risperidone for long acting injection (RC). There is only one injection per month, ambient temperature storage, flexibility interval dose and no requiring for an additional antipsychotic drug during the initiation.

The aim of the study was to observe how this new treatment has been established in our hospital and which place it takes in the therapeutic strategy of psychiatric disorders.

Setting and Method: All the patients who received PLP between February and June 2013 were included in this retrospective study. Data analysis consisted in collecting characteristics of the patients, terms of prescriptions (disease, initiation and maintenance dosage regimen of PLP, previous treatment), safety and tolerance. We used a prescription software and our electronic healthcare record to recover these data.

Results: In our hospital, 70 patients started a treatment by PLP during 5 months, the average age is $38.5( \pm 11.7)$ years with a sex ratio M/F of 2.3. For 23 patients it's a switch from RC to PLP in maintenance dose. In this situation the recommended dose scheme is respected in $70 \%$ of cases. For other patients who were previously treated by oral risperidone, the one week initiation dosing (PLP $150 \mathrm{mg}$ at day 1 and PLP $100 \mathrm{mg}$ at day 8 ) is realised in $95 \%$ of cases. Furthermore, in this group, there is a correlation between the dose of oral Risperidone and the maintenance dose of PLP. Finally, concerning data of efficiency, at this day $25 \%$ of patients who received PLP have stopped their treatment.

Conclusions: Despite a good tolerance, and clinical results as good as with RC, the number of prescriptions of PLP is significantly lower, this is unexpected because PLP has some practical benefits. This study have to be continued in order to verify these first data and to see if clinicians take some new prescriptions habits with their own experience.

Disclosure of Interest: None Declared.

\section{PT32}

Comparison outcomes effect of Amlodipine and Perindopril in geriatricpatients

A. A. Altemimi ${ }^{1}$, A. Sarriff ${ }^{1}$, H. A. Abdulrazzaq ${ }^{1}$, A. H. Al-taie ${ }^{2, *}$, O. L. Meng ${ }^{3}$

${ }^{1}$ Clinical Pharmacy, School of Pharmacy, Universiti Sains Malaysia, Pulau Pinang, Malaysia, ${ }^{2}$ Clinical Pharmacy, University of MarmaraCollege of Pharmacy, Istanbul, Turkey, ${ }^{3}$ Medical Department, Hospital Pulau Pinang, Pulau Pinang, Malaysia

Background and Objective: To evaluate the effectiveness of amlodipine and perindopril respectively alone or as combination use to achieve the targets of blood pressure in elderly hypertensivepatients with diabetes mellitus and dyslipidemia.

Setting and Method: It is retrospective cohort study for elderly patients who used amlodipine and perindopril or both for last 4 years depending on medical records of Medical Follow Up Clinic (MFUC) 
in Penang General Hospital, Malaysia. Prescription records used to evaluate the systolic blood pressure (SBP), diastolic blood pressure (DBP) and pulse rate (PR) in presence of the concomitant diseases in elderly patients. According to JNC VII and Malaysian Clinical Practice Guideline, the current sample was divided into three groups depending on age; from 65 to 74 years, from 75 to 84 years, and from 85 to 95 years old.

Main outcome measures: For elderly patients with SBP between 160 and $180 \mathrm{~mm} \mathrm{Hg}$, the blood pressure should be reduced by $20 \mathrm{~mm} \mathrm{Hg}$, while it should be $<160 \mathrm{~mm} \mathrm{Hg}$ for those with SBP $>180 \mathrm{~mm} \mathrm{Hg}$. Targets of blood pressure control of patients with; uncomplicated hypertension should be $<140 / 90$, high risk group (diabetes and history of cardiovascular diseases) should be $<130 / 80$, and diabetes with proteinuria of $>1 \mathrm{~g} / 24 \mathrm{~h}$ should be $<125 / 75$.

Results: Out of 3,200 patients, 405 patients were elderly with concomitant diseases that used antihypertensive. The total out of 405 patients, 155 patients revealed that 50,68 , and 37 elderly patients in the respective age group were prescribed amlodipine in the dosage ranges between 5 to $10 \mathrm{mg}$, while 186 patients as 69,68 and 49 patient were prescribed perindopril in the dosage range of 2 to $8 \mathrm{mg}$, respectively. All of them had the concomitant diseases of diabetes mellitus and dyslipidemia. The blood pressure outcome for SBP/DBP was $132 \pm 14 / 74 \pm 7$ and pulse rate was $72 \pm 8$. Combination antihypertensive therapy was significantly $(p<0.05)$ more effective toward SBP and DBPthan monotherapy.

Conclusions: Utilization of both amlodipine and perindopril may reduce the risk for cardiovascular complications geriatric patients. It encouraged following the international or local guideline when co-prescribing the two or more medications to elderly patients. Disclosure of Interest: None Declared.

\section{PT33}

Pharmacotherapeutic interventions in the neurology sector of a general hospital: results of one year of a clinical pharmacy service

J. P. V. Rodrigues ${ }^{1}$, L. P. Primo ${ }^{2}$, L. R. Ayres ${ }^{1, *}$, L. R. L. Pereira ${ }^{1}$, A. C. Abramovicius ${ }^{2}$

${ }^{1}$ Pharmaceutical Services and Clinical Pharmacy Research Center, School of Pharmaceutical Sciences of Ribeirão Preto, University of São Paulo, ${ }^{2}$ General Hospital of the School of Medicine of Ribeirão Preto, University of São Paulo, Ribeirão Preto, Brazil

Background and Objective: Neurological diseases are common in the population and there is a lack of well defined protocols for the treatment of some of them. The management of drug therapy is also difficult in relation to toxicity, drug interactions and complexity of treatment. Clinical Pharmacy (CP) is a tool that can provide safe and effective therapies to hospitalized patients. The aim of this study is to describe the pharmacotherapeutic interventions (PIs) conducted by clinical pharmacists and the degree of acceptance by physicians during the first year of this service implementation.

Setting and Method: This study was performed in the Neurology Sector of the General Hospital of Ribeirão Preto. We retrospectively revised pharmacists' intervention records from 109 hospitalized patients between December, 2011 and December, 2012. We included adults of both sexes, regardless of the reason of admission. The interventions performed by the pharmacists were mainly by oral communication with the physicians and when this was not possible, the recommendations were registered on the medical records.

Main outcome measures: It was quantified the PIs performed together with the medical staff that was documented. The PIs were classified in: dose change, discontinuation due to adverse reactions and/or drug interactions, change in the forms of administration, unnecessary discontinuation of and/or ineffective treatment, change in the treatment period, introduction of a new drug, drug or treatment change and pharmaceutical formulation change.

Results: The CP team made 74 PIs, of which 64 (86\%) were accepted by the physicians. There were 16 dose changes, 12 discontinuations due to adverse reactions and/or drug interactions, 18 changes in the forms of administration, nine unnecessary discontinuations of and/or ineffective treatments, two changes in the treatment period, four introductions of a new drug, nine drug or treatment changes and four pharmaceutical formulation changes.

Conclusions: The results show that $\mathrm{CP}$ is an important tool for the safety and effectiveness of the treatment of hospitalized patients, which is confirmed by the high degree of adherence from the medical team to the PIs performed.

Disclosure of Interest: None Declared.

\section{PT34}

Does omalizumab improve asthma control and health-related quality of life in patients with difficult-to-control severe allergic asthma in real life? A case report

C. Lambert ${ }^{1,2}$, S. Chanoine ${ }^{1,2,3}$, C. Chapuis ${ }^{1}$, A.-L. Provent ${ }^{1}$, S. Motyka ${ }^{1}$, C. Pison ${ }^{2,4,5}$, B. Allenet ${ }^{1,2,6}$, P. Bedouch ${ }^{1,2,6, *}$

${ }^{1}$ Pharmacy department, Grenoble University Hospital, ${ }^{2}$ Joseph Fourier University, ${ }^{3}$ Institut Albert Bonniot, INSERM U823, ${ }^{4}$ Pneumology department, Grenoble University Hospital, ${ }^{5}$ INSERM U1055, ${ }^{6}$ TIMC-IMAG UMR 5525/Themas, CNRS, Grenoble, France

Background and Objective: Achieving and maintaining optimal asthma control is a major goal of asthma management. Uncontrolled asthma has a high prevalence with major consequences on morbidity, quality of life, and economic burden. Despite an optimal adequacy and adherence to treatment, severe difficult-to-control asthma has been reported in a part of asthmatic population. Recently, omalizumab $\left(\right.$ Xolair $\left.^{\circledR}\right)$, anti-IgE monoclonal antibody, used as an add-on therapy alongside standard treatments, has been shown to decrease the risk of hospitalization or emergency department (ED) visits in patients with uncontrolled severe asthma in real-life practice.

Results: A 38-year-old man (BMI: $29.4 \mathrm{~kg} / \mathrm{m}^{2}$ ) was admitted to pneumology ward for asthma exacerbation. After childhood asthma remission, asthma reappeared at 34 years old, as a persistent severe asthma (predicted $\mathrm{FEV}_{1} / \mathrm{FVC}$ : $71 \%$ ) with allergy (overall $\mathrm{IgE}$ : $285 \mathrm{UI} / \mathrm{mL})$, and hypereosinophilia $(0.8 \mathrm{G} / \mathrm{L})$. A maintenance therapy by fluticasone and salmeterol $(500 / 50 \mu \mathrm{g}$ once daily) was introduced. Despite an optimal adherence, the patient was admitted twice in $\mathrm{ED}$ in the following year (predicted $\mathrm{FEV}_{1} / \mathrm{FVC}: 26 \%$ ), leading to the addition of oral corticotherapy ( $40 \mathrm{mg}$ daily) and indoor environmental counseling. Because of the lack of clinical improvement, omalizumab therapy was introduced $(300 \mathrm{mg}$ twice a month). Only 3 hospitalizations have been reported during the 2 next years without ED admission. However, no significant benefit was observed regarding to asthma control (ACT score: 14/25) and health-related quality of life (HRQL) (i.e., sleep disorders, limitations during daily life activities, corticosteroid side effects). Omalizumab therapy was stopped and 6 hospitalizations, including $5 \mathrm{ED}$ visits, were reported during the 10 following months after discontinuation, with a lung function decline (predicted $\mathrm{FEV}_{1} / \mathrm{FVC}$ : $54 \%)$ and a persistent IgE high level $(196 \mathrm{UI} / \mathrm{mL})$. In this context, omalizumab was reintroduced associated with a high level of maintenance treatment.

Conclusions: This case report supports that omalizumab decreases the risk of hospitalizations or ED visits in patients with difficult-tocontrol severe allergic asthma in population. Some studies promote a global benefit of omalizumab on asthma control and HRQL outcomes. 
However, this case report suggests that this therapy may be insufficient to control some aspects of asthma and to improve significantly HRQL in some phenotypes of severe allergic asthma.

Disclosure of Interest: None Declared.

\section{PT35}

Usage of antioxidants (vitamin $\mathbf{E}$ and $\mathrm{C}$ ) for cardiac cell protection in breast cancer patients against the toxicity of doxorubicin

\author{
A. H. Al-Taie ${ }^{1,2, *}$ \\ ${ }^{1}$ Clinical Pharmacy, Al-Mustansiryia University-College \\ of Pharmacy, Baghdad, Iraq, ${ }^{2}$ Clinical Pharmacy, Marmara \\ University-College of Pharmacy, Istanbul, Turkey
}

Background and Objective: Breast cancer is the most common cancer among females. Doxorubicin is used to treat breast cancer but has many toxic effects by the production of harmful free radicals to the different body systems including the heart. In order to reduce these serious oxidative effects, natural body defense mechanisms are liberated to protect agains this damage. This study designed to evaluate and report the external usage of antioxidants including vitamin $\mathrm{E}$ alone and in combination with vitamin $\mathrm{C}$ for cardiac cells protection against doxorubicin-free radicals production in females with breast cancer.

Setting and Method: This trial clinical study was carried out on 32 females with different stages of breast cancer after performing mastoctomy in Baghdad Teaching Hospital, Iraq. Patients were received two different systemic chemotherapeutic regimens IV infused every 21 days, the first one includes doxorubicin $60 \mathrm{mg} / \mathrm{M}^{2}$ in addition to cyclophosphamide, while the second one includes the same first combination in addition to florouracil. Three groups of patients were involved the first group(10) is A group and never receive any antioxidants during their therapy, the second one(12) is B group and received only a daily dose of $800 \mathrm{IU}$ vitamin $\mathrm{E}$ for a duration of 42 days, the last group(10) received the same dose of vitamin $\mathrm{E}$ besides $1 \mathrm{~g}$ daily of vitamin $\mathrm{C}$ for the same duration during their chemotherapy period. Study results were expressed as mean $\pm \mathrm{SD}$, Student's $t$-test and ANOVA to check the significance as $P$ value $<0.05$.

Results: The study showed that the doxorubicin therapy results in elevated serum levels of CPK after 21 and 42 days respectively compared to baseline $(P<0.05)$. Serum CPK level is significantly reduced after 21 days with vitamin $\mathrm{E}$ therapy when compared with doxorubicin therapy $(P<0.05)$. Therapy with a combination of antioxidant drugs (vitamins $\mathrm{E}$ and $\mathrm{C}$ ) produced a significant reduction in CPK serum level after 21 days as compared with the baseline $(P<0.05)$. A reduction was also observed when compared with both groups of doxorubicin therapy as well as with vitamin E therapy at the same period $(P<0.05)$. Doxorubicin therapy also results in elevated serum levels of LDH after 21 and 42 days compared to baseline $(P<0.05)$. Serum LDH levels are significantly reduced after 21 and 42 days respectively with a combination of vitamins $E$ and $C$ therapy when compared with both groups of doxorubicin therapy as well as vitamin $\mathrm{E}$ therapy $(P<0.05)$.

Conclusions: Doxorubicin produce free radicals that attenuate antioxidant defense mechanism of the body leading to several toxic effects on the heart. The use of antioxidant drugs as vitamin $\mathrm{E}$ alone or a combination of antioxidants (vitamins $\mathrm{E}$ and $\mathrm{C}$ ) may protect against the toxic damaging effects of doxorubicin on the heart and this protection is found to be superior to that observed with vitamin $\mathrm{E}$ therapy alone which is dose, time and combination dependent on lowering serum levels of CPK and LDH which are indicators of heart damage.

Disclosure of Interest: None Declared.

\section{PT36}

Doxorubicin-induced hepatic and renal cellar damage could be protected by the usage of antioxidant agents (vitamin $\mathrm{E}$ and $\mathrm{C}$ ) in breast cancer patients

A. H. Al-Taie ${ }^{1,2, *}$, H. A. Abdulrazzaq ${ }^{3}$

${ }^{1}$ Clinical Pharmacy, Marmara University-College of Pharmacy, Istanbul, Turkey, ${ }^{2}$ Clinical Pharmacy, Al-Mustansiryia UniversityCollege of Pharmacy, Baghdad, Iraq, ${ }^{3}$ Clinical Pharmacy, School of Pharmaceutical Science, University of Science Malaysia, Pulau Pinang, Malaysia

Background and Objective: Breast cancer is globally the most common cancer worldwide. One of the most common medicines used for breast cancer therapy associated with adverse effects is doxorubicin by free radicals production affect different body systems including the liver and kidney. To reduce these oxidative effects, natural body defense mechanisms including besides external protectants including vitamin $\mathrm{E}$, vitamin $\mathrm{C}, \beta$-carotene are involved.

Setting and Method: A randomized trial on 30 breast cancer females after performing mastoctomy in Baghdad Teaching Hospital, Iraq was done with two various IV systemic chemotherapeutic regimens administered every 21 days, the initial one was with doxorubicin $60 \mathrm{mg} / \mathrm{M} 2$ plus cyclophosphamide, while the second one was the same first combination plus florouracil. 10 patients group on chemotherapy alone, 10 patients group on 800 IU vitamin $\mathrm{E}$ for a duration of 42 days, and 10 patients group on the same dose of vitamin $\mathrm{E}$ besides $1 \mathrm{~g}$ daily of vitamin $\mathrm{C}$ for 42 days. This study was approved by the scientific committee of the hospital and all patients were signed the related consent form. The results were expressed as mean $\pm \mathrm{SD}$, Student's $t$ test and ANOVA to check the significance as $P$ value $<0.05$.

Results: A combination of vitamins $\mathrm{E}$ and $\mathrm{C}$ reduced serum level of AST compared with doxorubicin therapy. Serum TSB level is significantly reduced after 21 days with vitamin E therapy $(P<0.05)$. Serum TSB levels are significantly reduced after 21 and 42 days respectively with vitamin $\mathrm{E}$ therapy $(P<0.05)$. Therapy with a combination of(vitamins $\mathrm{E}$ and C) produced a significant reduction in TSB serum level after 21 days as compared with the baseline $(P<0.05)$. Doxorubicin therapy results in elevated serum levels of urea after 21 compared to baseline $(P<0.05)$. Serum urea level is significantly reduced after 42 days compared with baseline in patients treated only with vitamin $\mathrm{E}(P<0.05)$. With a combination of vitamins $\mathrm{E}$ and $\mathrm{C}$ therapy, serum urea levels were reduced significantly after 21 and 42 days period $(P<0.05)$. Serum creatinine level was significantly increased with doxorubicin administration after 42 days of therapy compared to baseline $(P<0.05)$. Therapy with both of vitamins $E$ and $C$ resulted in significant reduction of serum creatinine levels after 21 and 42 days respectively compared to baseline and a significant reduction after 42 days compared to doxorubicin therapy $(P<0.05)$.

Conclusions: Doxorubicin retards antioxidant defense mechanism of the body leading to several toxic effects on the liver and kidney. The use of vitamin $\mathrm{E}$ alone or a combination of antioxidants (vitamins $\mathrm{E}$ and $\mathrm{C}$ ) may protect against this toxic damage, and this protection is superior to that with vitamin E therapy alone which is dose, time and combination dependent on lowering serum levels of ALT, TSB, urea and creatinine which are indicators of liver and kidney damage respectively.

Disclosure of Interest: None Declared.

\section{PT37}

\section{Compliance to antihypertensive and antidiabetic medications}

\section{J. Gypens ${ }^{1, *}$, F. Wirth ${ }^{2}$, L. M. Azzopardi ${ }^{2}$, S. Steurbaut ${ }^{1}$, S. Fava ${ }^{3}$}

${ }^{1}$ Vrije Universiteit Brussel, Brussels, Belgium, ${ }^{2}$ Department of Pharmacy, Faculty of Medicine and Surgery, University of Malta, ${ }^{3}$ Diabetes and Endocrine Centre, Mater Dei Hospital, Msida, Malta 
Background and Objective: Good pharmacological compliance is important in the management of hypertension and diabetes mellitus to maintain optimal blood pressure and metabolic control, reducing risk of complications. The objective of this study was to evaluate compliance to antihypertensive and antidiabetic medications.

Setting and Method: Four-week study at Diabetes and Endocrine Centre, MDH. Patients recruited were asked to complete a selfadministered questionnaire, including 7 'Yes and No' questions and 1 'multiple choice' question on pharmacological compliance ('Morisky test'). Each question was given a score of 1 or 0 , depending on the question. A total score of 0 was classified as 'high', 1-2 'medium' and $>2$ 'low' compliance.

Main outcome measures: Categorisation of patients into 'high', 'medium' or 'low' medication compliance score.

Results: 125 patients participated; $55 \%$ were male, most (49\%) were between 50 and 59 years, most $(41 \%)$ had a primary education, the majority $(52 \%)$ were retired and $87 \%$ did not live alone. A 'high' compliance score was obtained by $43 \%$ of patients, while $31 \%$ and $26 \%$ obtained a 'medium' and 'low' compliance score respectively. $98 \%$ of patients claimed to have taken all their medications the day before the questionnaire was distributed. $70 \%$ of patients said that they do not frequently forget to take their medications, $82 \%$ said that over the previous 2 weeks they did not miss any medication doses, $92 \%$ of patients never stopped their medication without consulting their physician even when they felt worse, $85 \%$ of patients do not forget to take their medications with them when travelling or going to work and $74 \%$ of patients do not feel stressed to comply with their treatment plan.

Conclusions: This study showed that most patients showed a 'high' pharmacological compliance. Systems to identify the 'medium' and 'low 'compliers should be adopted so that pharmacists in this setting could support patients to improve compliance.

Disclosure of Interest: None Declared.

\section{Public health}

\section{PH01}

Patient controlled analgesia: regional guidelines for good use

M. Lefebvre-Caussin'1 , C. Borel ${ }^{1, *}$, E. Guedon ${ }^{2}$, S. Gervais ${ }^{2}$, C. Beuzelin' ${ }^{2}$, J. Delesque ${ }^{2}$, G. Canchon ${ }^{3}$, J. Doucet ${ }^{1}$, E. Remy ${ }^{1}$

${ }^{1}$ OMEDIT of Upper-Normandy, ${ }^{2}$ Palliative care, ${ }^{3}$ Pharmacy, Hospital University, Rouen, France

Background and Objective: Patient Controlled Analgesia (PCA) allows to optimize therapeutic pain management. However, the use of an infusion pump for PCA is complex and ignorance of this device can limit its use. Concern has been raised about the high incidence of critical incidents related to these devices. The objective of this regional work, intended for doctors and nurses, hospital workers or liberals, is to promote the good use of PCA.

Setting and Method: An expert workgroup, composed of doctors, pharmacists and nurses, worked on a regional guidelines for PCA use, made of recommendations and practical tools.

Results: Five issues are mentioned in the regional guidelines: prescription, preparation, administration, infusion assembly, patient's and infusion's supervision, and patient information. For each issue, several help tools are associated to the recommendations. Templates of prescriptions (for narcotics, medical equipment or nursing act), an opioids equivalence chart, a table of the main molecules used in PCA (specifying the various dosages), the pharmacy or hospital pharmacy's supply arrangements and product stability in pump for PCA are proposed. A template of treatment supervision form allows to confront the prescription parameters with the efficiency and tolerance evaluation of treatment. A practical tool is proposed to help nurses in calculating doses, concentration, infusion rates and choosing the best opioids presentation. The nursing practice of having a second person repeat the calculation should be adopted. A sketch of the device reminds the importance of antireflux valve which should be inserted in any other line that is connected to the PCA, in order to prevent back-flow up. A protocol of opioids overdose management is also proposed, as well as an information sheet intended to patients, mentioning the steps to follow in case of adverse event or in case of unrelieved pain.

Conclusions: To complete these guidelines, an evaluation of professional practices was proposed to make all healthcare professionals aware of these issues and help them in the improvement of their practices. An internet-base training (e-learning) on PCA is being developed to promote the training of healthcare professionals.

Disclosure of Interest: None Declared.

\section{PH02}

Potentially inappropriate prescriptions in immobilized patients: applicability of beers criteria

J. Alonso Herreros ${ }^{1, *}$, M. Saez Fajardo ${ }^{1}$, A. Gonzalez Cuello ${ }^{2}$, J. Abellon Ruiz ${ }^{3}$

${ }^{1}$ Pharmacy, Hospital General Universitario Reina Sofia (Murcia), ${ }^{2}$ Pharmacology, Universidad De Murcia, Murcia, ${ }^{3}$ Pharmacy, Hospital Los Arcos Mar Menor, Santiago De La Ribera, Spain

Background and Objective: Immobilized-patient(IP) is defined as a patient who spends most of their time in bed, or has significant impaired physical mobility, for a period of time longer than two months. In Spain there is a special care plan for these patients (PAPI). Our aim is to identify the type and prevalence of potentially inappropriate prescriptions(PIP) at IP, and know the applicability of Beers-criteria(BC) in this population.

Setting and Method: An observational and descriptive study was made by restrospective medical record reviwing. The sample size calculation was performed for a total population of 1,194 patients included in the PAPI of our teaching hospital, with an error rate of $7.5 \%$, a confidence interval of $95 \%$ and a response distribution of $40 \%$. Patients were randomly selected among the various health centers depending on the hospital, in proportion to the total number of patients included in the PAPI in each center. The BC2012 were applied to patients over 65 years old, with more than five drugs prescribed, and at least two diagnoses.

Main outcome measures: Potentially inappropriate prescription in IP. Implementation of the BC in IP.

Results: The $69 \%$ from IP met the BC .

Of the patients who met the BC the $69.33 \%$ were women $(\mathrm{n}=104)$ and $30.66 \%$ were male $(\mathrm{n}=46)$. The mean age was $82.37 \pm 1.06$ years in women and $82.15 \pm 1.065$ in men.

The mean number of drugs prescribed by patient was 11.44 medications.

The prevalence of PIP was of a $92.67 \%$. That is, of the 150 patients analyzed, only 11 did not receive any PIP according to the BC. The largest proportion of PIP was detected in the group of benzodiazepines, followed by the NSAID group. Only 10 drugs were implicated on the $55.2 \%$ of the PIP.

Conclusions: There is a high percentage of IP that meets BC. The PIP are more common (92\% vs 30-40\%) in this subgroup, than among other subgroups of elderly patients. Thus, the IP must be a group of special interest for the pharmaceutical care plan.

Disclosure of Interest: None Declared. 


\section{PH03}

One year of off-label prescriptions at regional level: focus on cancer drugs

C. Borel $^{1, *}$, F. Sohouenou ${ }^{1}$, M. Lefebvre-Caussin ${ }^{1}$, D. Fuss ${ }^{2}$, F. Basuyau ${ }^{3}$, M. Fontaine-Adam ${ }^{2}$, J. Doucet ${ }^{1}$, E. Remy ${ }^{1}$

${ }^{1}$ OMéDIT of Upper Normandy, ${ }^{2}$ Pharmacy, University Hospital, ${ }^{3}$ Pharmacy, Cancer centre Henri Becquerel, Rouen, France

Background and Objective: Currently, the French regulatory context and health agencies request an evaluation of prescription practice. To promote the appropriate use of medicines, data on off-label prescriptions have been collected in the region during the year 2012. It aims at comparing practices to reference documents, identifying specific off-label emergent situations and thereby participating in healthcare vigilance and avoid misuse situations.

Setting and Method: The OMéDIT (a regional health observatory) collected, during 2012, off-label prescriptions of expensive medicinal products through a declarative data reporting process from regional hospitals which have signed a contract on appropriate use of medicines.

Requested items were indication of prescribed drugs, justification of prescriptions (bibliography and/or clinical argument) and decision process (multidisciplinary meeting or not).

Results: In 2012, 420 declarations from 14 hospitals have been reported to the OMéDIT. $76 \%$ (318) of them related to cancer drug and the other part (non cancer drugs) predominantly involved erythropoietin $(75 \%)$, infliximab $(7 \%)$ and immunoglobulins $(5 \%)$. With respect to cancer drugs, $59 \%$ of off-label prescriptions were pemetrexed, mainly used in association with carboplatin for nonsmall cell lung cancer treatment. Rituximab was the second most frequent off-label cancer drug use (15\%) followed by bendamustine, bortezomib and bevacizumab (5-7\%).

None of the 318 cancer drug off-label prescriptions was judged as non acceptable according to references of National Cancer Institute. $67 \%$ of these prescriptions were classified by the French National Agency for Medicines and Health Products Safety as situations for which data were "not sufficient to decide". The remaining $33 \%$ were not listed in the guidelines. Only 3 patients benefited from a "temporary protocol of treatment" for mesothelioma by pemetrexed and carboplatin.

However, all the cancer drug off-label prescriptions have been justified by oncologists either by a clinical justification $(53 \%)$ or bibliography $(40 \%)$ or two of them $(7 \%)$.

Conclusions: These 2012 regional results on off-label prescriptions have allowed to perform an innovation watch through new bibliographic data. These results have been sent to regional health establishments and also to regulatory agencies for participation in the update of national reference documents. In the same time, a form which can colligate off-label use informations is in progress at national level.

Disclosure of Interest: None Declared.

\section{PH04}

Use of psycho-stimulants among university students: role of awareness

A. Qanbar ${ }^{1, *}$, A. Qanbar ${ }^{1}$, on behalf of Ahmed Majed Qanber, Ghadeir Abd Al Naby Allam, Maha Saber-Ayad

${ }^{1}$ College of Pharmacy, University of Sharjah, Sharjah, United Arab Emirates

Background and Objective: Psycho stimulant drugs are prescribed for specific medical conditions, but they may be abused among college students, mainly for performance enhancement during exams period. Pharmacists could help increasing the awareness and educating the University students about the risks of this drug abuse. The Aim of the study was to measure the magnitude of abusing psychostimulants among university students and to evaluate the effect of a short awareness session.

Setting and Method: The study included 200 students either abusing the drug or vulnerable to abuse them if they have the chance. First a survey was done to collect data. Then, an intervention (awareness posters, brochures, an article in the University magazine and discussions) was conducted. At the end, a second survey was distributed aiming to test the impact of the awareness session.

Main outcome measures: The Aim of the study was to measure the magnitude of abusing psychostimulants among university students and to evaluate the effect of a short awareness session.

Results: Out of 200 students, $7 \%$ had used a psychostimulant before, $24 \%$ are willing to use it if available. Three out of 7 abusers decided to stop the psychostimulants $(44.5 \%)$ after the awareness session. Abuse vulnerability was reduced from 24 to $15.6 \%$ after the session. Conclusions: Psychostimulant abuse is a significant problem among University students in the UAE. Short term awareness programs using various tools and conducted in the university may help in reducing the risk of psychostimulant abuse.

Disclosure of Interest: None Declared.

\section{Research development}

\section{RD02}

A medication assessment tool for community acquired pneumonia (MAT-CAP): design and validation

\section{J. U. Høgli ${ }^{1, *}$, L. Småbrekke ${ }^{1}$, B. Garcia ${ }^{1}$ \\ ${ }^{1}$ Department of Pharmacy, University of Troms $\varnothing$, Troms $\varnothing$, Norway}

Background and Objective: A Medication Assessment Tool (MAT) comprises a number of explicit review criteria (RC) based on clinical guideline recommendations for treatment of a specific disease, and is used to identify clinical improvement potentials. Several MATs have been described in literature. To our knowledge a MAT to assess guideline adherence for the treatment of community acquired pneumonia (CAP), a disease with high morbidity and mortality, has not been published. We aimed to develop and validate a MAT to assess treatment of patients admitted to hospital with CAP (MAT-CAP). Setting and Method: We selected potential quality indicators (QI) from local, national and international clinical practice guidelines. Senior consultants from the University Hospital of Northern Norway (UNN) joined a validation group to demonstrate content validity using a two-round modified Delphi technique. Consensus threshold was set to $\geq 75 \%$ agreement. Before further testing, each QI was reformulated to RC that can be answered with "YES" or "NO", reflecting adherence and complying with MAT methodology structure. The MAT-CAP was applied on 84 patient profiles containing information extracted from the patient records of CAP patients admitted to UNN in 2008. Inter- and intra-rater reliability was investigated using Cohen's kappa ( $\kappa)$. For reliability $\kappa \geq 0.75$ was considered excellent agreement. Feasibility with applicability and application time, adherence to guideline recommendations and the RCs potential for improvement were informed. Threshold for acceptable applicability was set to $>1 \%$ and threshold for no potential for improvements was set at adherence $>85 \%$.

Main outcome measures: QIs reflecting guideline recommendations, percent agreement of the QIs in the validation group, inter and intrarater reliability, percent applicability, application time, percent 
adherence to guideline recommendations and the RCs potential for improvement.

Results: Sixteen QIs were selected from four different clinical practice guidelines. The QIs covered areas as initial empirical treatment, pathogen specific treatment, dose adjustment according to renal function, switch from intravenous to per oral treatment and treatment duration. Six experts (infection medicine, medical microbiology or internal medicine) comprised the final validation group. All 16 QIs achieved consensus, albeit two were finally merged. Cohen's kappa for the overall inter- and intra-rater reliability was 0.88 and 0.95 , respectively. Overall applicability was $52.7 \%$. Only the RC concerning penicillin allergy was not applicable. Overall adherence for 887 applicable criteria was $56.7 \%$. Three criteria had no potential for improvement (the criteria for documentation of amendment of empirical treatment, microbiological sampling and dose adjustment according to renal function). Mean application time was 3.08 and $3.76 \mathrm{~min}$ for rater 1 and 2, respectively.

Conclusions: We developed a MAT-CAP with 15 criteria and demonstrated its content validity, reliability and feasibility.

Disclosure of Interest: None Declared.

\section{RD03}

Development of a survey to evaluate safe medication practices in Portuguese hospitals: interim findings from a national Delphi panel

S. Oliveira-Martins' ${ }^{1}$, C. T. Barros ${ }^{1}$, A. Coelho ${ }^{2}$, E. Domingues ${ }^{3}$, F. Duarte Ramos ${ }^{1}$, M. J. Gaspar ${ }^{2}$, A. Graça ${ }^{4}$, F. Haimé ${ }^{5}$, D. Paulino ${ }^{6}$, M. Placido ${ }^{7}$, A. W. Silva ${ }^{7}$, M. P. Guerreiro ${ }^{8}$;"

${ }^{1}$ Social Pharmacy Department, Faculty of Pharmacy, Lisbon University, iMED.UL, ${ }^{2}$ Direcção Geral de Saúde, ${ }^{3}$ Faculty of Pharmacy, Lisbon University, ${ }^{4}$ Escola Superior de Tecnologia da saúde de Lisboa, Lisbon, Portugal, ${ }^{5}$ University of Utrecht, Utrecht, Netherlands, ${ }^{6}$ ISCSEM, ${ }^{7}$ Social Pharmacy Department, Faculty of Pharmacy, Lisbon University, ${ }^{8}$ ISCSEM, iMED.UL, Lisbon, Portugal

Background and Objective: Adverse drug events (ADE) are a significant inpatient problem, resulting in human suffering, increased healthcare cost and loss of trust in health professionals. Measuring safe medication practices is an important step towards improvement. International instruments for this purpose were not entirely suitable to the Portuguese reality. This study, which is part of a larger research programme, aims to determine the face and content validity of an instrument to measure safe medication practices in Portuguese hospitals.

Setting and Method: Hospital setting. Design: Two-round multidisciplinary Delphi panel. Experts were defined as a health professional working in a hospital setting and dealing with medicines on a regular basis. Professional credentials and/or status within the profession were additionally used to identify experts. To ensure variability within the panel, experts were recruited in hospitals from different regions, varying in size and university affiliation. The survey included 128 items respecting to individuals, processes of care and the organisation, adapted from international instruments or derived from the literature and national guidelines. Each item was rated for relevance in a scale ranging from 1 (definitely not relevant) to 7 (definitely relevant). Consensus was defined at the outset (positive consensus-items with $75 \%$ or more respondents scoring 6 or 7 ; negative consensus-items with $75 \%$ or more respondents scoring 1 or 2).

Main outcome measures: Relevance of survey items (median score), Number of survey items reaching consensus.

Results: One hundred and thirty five experts were invited, of which 95 completed the first round ( 33 nurses, 22 pharmacists, 23 pharmacy technicians, 17 physicians). Overall experts agreed with the relevance of survey items; the median score for $62 \%(80 / 128)$ of the items was 7 whilst $34 \%(43 / 128)$ had a median score of 6 . Positive consensus was obtained in 91 items; no negative consensus was found. Based on experts' suggestions, eight new items were included in the second round, which is currently underway.

Conclusions: A large number of items reached the pre-defined level of positive consensus in round one, suggesting face and content validity for inclusion in a future survey to measure safe medication practices in Portuguese hospitals.

Disclosure of Interest: None Declared.

\section{RD04}

Pharmacy practice in the North East of Scotland: a case study of digital literacy

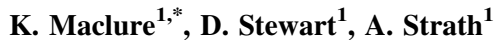 \\ ${ }^{1}$ School of Pharmacy and Life Sciences, Robert Gordon University, \\ Aberdeen, United Kingdom
}

Abstract submitted before to: Royal Pharmaceutical Society Conference 2013 (poster).

Background and Objective: Scottish Government health policy promotes information technology (IT) to support healthcare practice. Pharmacy relies on IT to provide patient care in partnership with other healthcare professionals. Pharmacy team member's ability to use IT at home and at work is known as digital literacy which is identified as a key skill by the World Health Organization, European Parliament and features in UK National Occupational Standards for health. The aim of this research was to explore the digital literacy related training experiences and needs of the pharmacy team.

Setting and Method: Mixed methods were applied in a multiple case study. Pharmacies in the NE Scotland were purposively selected on setting, pharmacy management system implemented and type (single independent to large multiple; community or hospital). Quantitative data were gathered during the consent process with qualitative data recorded as observational/interview field notes. Consent forms included 4 demographic questions: sex, age band, role, pharmacy experience; final question, "As a gauge of your current IT experience, if you were to do a course, which of the following would be the most appropriate challenge for you?' followed by a list of 6 courses. Quantitative data were analysed using descriptive statistics in SPSS version 17.0. Qualitative data were analysed using a constant comparative approach to elicit themes. The study was approved by the Ethics Review Panel of RGU School of Pharmacy and Life Sciences. Main outcome measures: Pharmacy team member's self reported levels of digital literacy and themes related to their training experience and needs.

Results: Observations were conducted August 2012 to March 2013 in 17 community and 2 hospital pharmacies with 94 participants: 24 pharmacists including 2 locums; 2 pharmacy graduates; 19 pharmacy technicians; 15 dispensing assistants; 34 medicines counter assistants. Most frequently self selected IT course across all roles was 'Computing for the Quietly Confident' $(\mathrm{n}=39)$ followed by 'Computing for the Terrified' $(\mathrm{n}=19)$, together accounting for nearly twothirds of participants. The remainder selected European Computer Driving Licence (ECDL; $\mathrm{n}=14$ ), 'Computing for the Courageous' $(\mathrm{n}=13)$, ECDL Advanced $(\mathrm{n}=5)$ and 'Degree or Diploma' $(n=4)$. Emergent themes from community and hospital settings include training, team work, technology, usability and processes. Informal teaching and learning, shared experience, half measures in implementation, workarounds and resistance to change were reported with polarised views of technology evident. 
Conclusions: Pharmacy staff perceived their own digital literacy skills as basic with no formalised, related training. Increased reliance on IT in both community and hospital pharmacy may need to be formally reflected in future pharmacy curricula. Although limited by the unreliability of self reporting and potential recruitment, response and social desirability biases, these findings provide insight into a digital literacy related training gap in pharmacy practice.

Disclosure of Interest: None Declared. 